\title{
Level 3 Trigger Algorithm and Hardware Platform for the HADES Experiment
}

Inaugural-Dissertation

zur Erlangung des Doktorgrades der Naturwissenschaften der Justus-Liebig-Universität Gießen im Fachbereich 07

(Mathematik und Informatik, Physik, Geographie)

Oktober 2007

vorgelegt von

\section{DANiEl Georg Kirschner}

aus Frankfurt am Main

\section{JUSTUS-LIEBIG- (7) UNIVERSITAT}

II. Physikalisches Institut

Heinrich-Buff-Ring 16

D-35392 Gießen

Germany 
Dekan:

Prodekan:

I. Berichterstatter:

II. Berichterstatter weiterer Prüfer:
Prof. Dr. Bernd Baumann

Prof. Dr. Claus-Dieter Kohl

Prof. Dr. Wolfgang Kühn

Prof. Dr. Claus-Dieter Kohl

PD Dr. Stefan Leupold

Tag der mündlichen Prüfung: 2007-10-26 


\section{Zusammenfassung}

Eines der Hauptaufgabengebiete des HADES ${ }^{1}$ Experiments ist die Erforschung des Zerfalls leichter Vektormesonen in Leptonenpaare innerhalb eines dichten Mediums. Diese Zerfälle sind ein konzeptionell ideales Werkzeug zur Erforschung der invarianten Masse von Vektormesonen in einem Medium, da diese das Medium in dem sie zerfallen ohne weitere starke Wechselwirkung verlassen. Daher wird die Messung nicht von Wechselwirkungen im Endzustand beeinflusst. Unglücklicherweise sind die Verzweigungsverhältnisse von Vektormesonen nach Leptonenpaaren sehr klein $\left(\approx 10^{-5}\right)$, was es nötig macht Experimente mit hohen Reaktionsraten und hoher Akzeptanz durchzuführen. Zusätzlich verfügt das HADES Experiment über ein leistungsfähiges TriggerSystem, um die physikalisch relevanten Ereignisse in den aufgenommenen Daten anzureichern.

Das Augenmerk dieser Arbeit liegt in der Entwicklung der nächsten Generation eines in Echtzeit arbeitenden Trigger-Verfahrens für das HADES Experiment zur signifikanten Verbesserung der Anreicherung von Leptonen. Des weiteren wurde eine Hardwareplatform (das GE-MN²) zur Entwicklung und zur Erprobung des Trigger-Verfahrens entwickelt.

Das GE-MN System besitzt zwei Gigabit-Ethernet Schnittstellen für den Transport von Daten, eine VMEbus Schnittstelle zur Konfiguration und Überwachung sowie einen TigerSHARC DSP zur Datenverarbeitung. Es ermöglicht die Diskussion der Herausforderungen und Vorteile beim Einsatz eines solchen, auf Standard-Netzwerktechnologie aufbauenden, Systems in physikalischen Experimenten.

Die vorgestellte Trigger-Methode korreliert die Ring-Informationen des HADES RICH Detektors $^{3}$ mit den angesprochenen Drahtkammern des HADES MDC Detektors ${ }^{4}$. Sie berechnet die Drahtkammern, die durch das Signal eines hindurch fliegenden Leptons hätten ansprechen sollen und vergleicht diese mit den tatsächlich angesprochenen Drahtkammern. Die angesprochenen Drähte werden ausgehend von den Polar- und Azimutwinkelinformationen berechnet, indem ausgehend vom Target eine Gerade mit den Winkeln des Ringes in den Raum gelegt wird. Diese Gerade führt durch die inneren Drahtkammern. Die geschnittenen Drahtkammern sind diejenigen, die einen Treffer registrieren sollten. Zur Kompensation verschiedener Ungenauigkeiten werden nicht Treffer der berechneten Driftzellen gefordert, sondern lediglich Treffer in einem Korrelationsfenster verlangt, und nur ein Bruchteil der berechneten Drahtkammerlagen muss einen Treffer registriert haben.

Im Weiteren wird gezeigt, daß die Verwendung dieser Korrelationsmethode die Anzahl an Ereignissen, die Leptonen enthalten, um einen von dem Experiment abhängigen Faktor anreichern kann. Die möglichen Anreicherungsfaktoren für verschiedene Reaktionen sind $\approx 30$ für $\mathrm{p}+\mathrm{p}$, $\approx 50$ für $\mathrm{C}+\mathrm{C}$ und $\approx 5,5$ für $\mathrm{Ar}+\mathrm{KCl}$. Hierbei liegt die Effizienz immer über $80 \%$ und zeigt keine Abhängigkeit von den Größen Polarwinkel, Azimuthalwinkel und Leptonenimpuls. Es wird

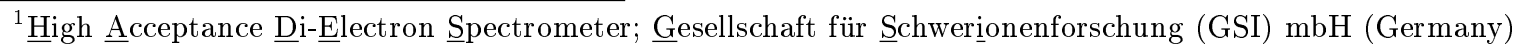

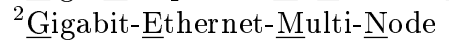

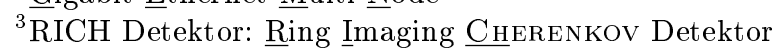

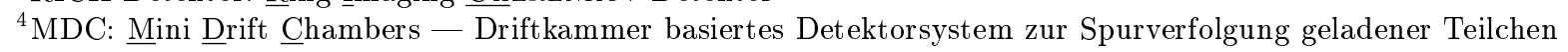


gezeigt wie die Einstellungen zum Betrieb der Trigger-Methode vollständig aus den in Echtzeit verfügbaren Rohdaten gewonnen werden kann.

Die Geschwindigkeit der Methode in analysierten Ereignissen pro Sekunde wird mit dem GEMN untersucht. Sie hängt ab von den Eingangsdaten und reicht von 6600 Ereignisse/s für p $+\mathrm{p}$ Daten bis zu 1400 Ereignisse/s für $\mathrm{Ar}+\mathrm{KCl}$ Daten unter Verwendung lediglich eines GE-MN Systems. 


\section{Abstract}

One focus of the HADES experiment ${ }^{5}$ is the investigation of the decay of light vector mesons inside a dense medium into lepton pairs. These decays provide a conceptually ideal tool to study the invariant mass of the vector meson in-medium, since the lepton pairs of these meson decays leave the reaction without further strong interaction. Thus, no final state interaction affects the measurement. Unfortunately, the branching ratios of vector mesons into lepton pairs are very small $\left(\approx 10^{-5}\right)$. This calls for a high rate, high acceptance experiment. In addition, a sophisticated real time trigger system is used in HADES to enrich the interesting events in the recorded data.

The focus of this thesis is the development of a next generation real time trigger method to improve the enrichment of lepton events in the HADES trigger. In addition, a flexible hardware platform (GE-MN) was developed to implement and test the trigger method.

The GE-MN features two Gigabit-Ethernet interfaces for data transport, a VMEbus for slow control and configuration, and a TigerSHARC DSP for data processing. It provides the experience to discuss the challenges and benefits of using a commercial standard network technology based system in an experiment.

The developed and tested trigger method correlates the ring information of the HADES RICH ${ }^{6}$ with the fired wires (cells) of the HADES $\mathrm{MDC}^{7}$ detector. This correlation method operates by calculating for each event the cells which should have seen the signal of a traversing lepton, and compares these calculated cells to all the cells that did see a signal. The cells which should have fired are calculated from the polar and azimuthal angle information of the RICH rings by assuming a straight line in space, which is starting at the target and extending into a direction given by the ring angles. The line extends through the inner MDC chambers and the traversed cells are those that should have been hit. To compensate different sources for inaccuracies not the exact cell numbers are required, but instead the cells in a matching window are investigated, and only a fraction of the MDC layers that should be hit are required to have seen a hit.

It is demonstrated that the use of the correlation method can enhance the number of events which contain leptons by an experiment dependent factor. The possible enhancements of different reactions are $\approx 30$ for $\mathrm{p}+\mathrm{p}, \approx 50$ for $\mathrm{C}+\mathrm{C}$ and $\approx 5,5$ for $\mathrm{Ar}+\mathrm{KCl}$. The efficiency at these enhancements is above $80 \%$, and it is constant in relation to the parameters polar angle, azimuthal angle and momentum. It is demonstrated how to obtain the correlation parameters from the online available raw data only.

The performance of the correlation method in terms of the events analyzed per second has been studied with the GE-MN. The performance depends on the input data and ranges from 6600 events/s for $\mathrm{p}+\mathrm{p}$ data to 1400 events/s for $\mathrm{Ar}+\mathrm{KCl}$ data for a single GE-MN system.

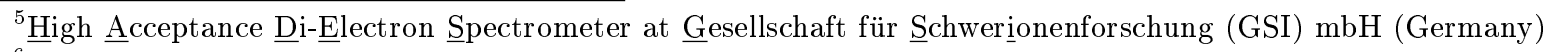

${ }^{6}$ RICH detector: Ring Imaging $\underline{\mathrm{CH}}$ EREN KOV detector

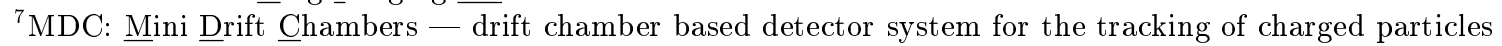




\section{Contents}

1. Motivation and Theory Overview 11

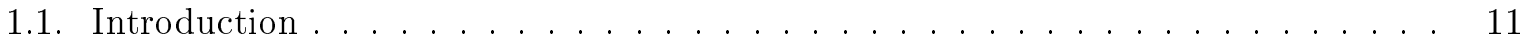

1.2. Quantum Chromodynamics - QCD . . . . . . . . . . . . . . 13

1.2.1. Asymptotic Freedom and Anti-Screening . . . . . . . . . . . . . 15

1.2.2. Confinement . . . . . . . . . . . . . . . . 16

1.2.3. Symmetry and Symmetry Breaking . . . . . . . . . . . . . . . . 18

1.2.4. Chiral Symmetry, its Breaking and Restoration . . . . . . . . . . . . 19

1.3. Subatomic Structure and Form Factors . . . . . . . . . . . . . . . . . . 23

1.4. Light Vector Mesons and Vector Meson Dominance . . . . . . . . . . . . . . 25

1.5. Dilepton Experiments . . . . . . . . . . . . . . . . . . 27

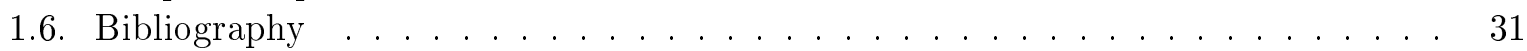

2. The HADES Spectrometer 35

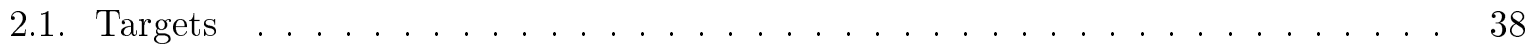

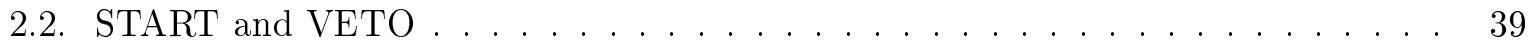

2.3. Ring Imaging C̆enenkov Detector - RICH . . . . . . . . . . . . . . . . 40

2.3.1. ČERENKOV Effect . . . . . . . . . . . . . . . . . . . . . . . . 40

2.3.2. The HADES RICH . . . . . . . . . . . . . . . . . . . . . 41

2.3.3. Online Ring Recognition . . . . . . . . . . . . . . . . . . . . . . 43

2.4. Mini Drift Chambers - MDC . . . . . . . . . . . . . . . . . 44

2.4.1. Physics and Characteristics of a Drift Cell . . . . . . . . . . . . . . . 47

2.5. Magnet System - ILSE . . . . . . . . . . . . . . . . . . . . . . . 49

2.6. Time of Flight Detector - TOF . . . . . . . . . . . . . . . . . 49

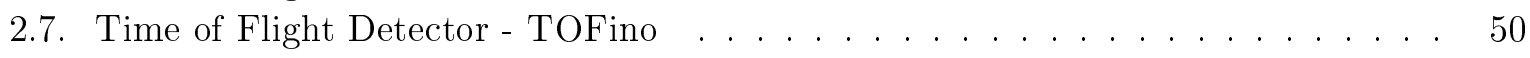

2.8. Pre-Shower Detector . . . . . . . . . . . . . . . . . 50

2.9. Resistive Plate Chambers Detector - RPC . . . . . . . . . . . . . 51

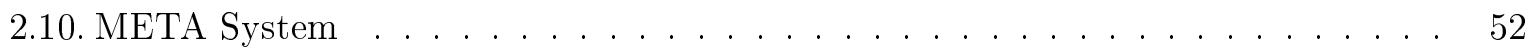

2.11. Tracking in the Magnetic Spectrometer . . . . . . . . . . . . . . 52

2.12. The Trigger System . . . . . . . . . . . . . . . . . . . 55

2.12.1. The First Level Trigger - LVL1 _ . . . . . . . . . . . . . . . . . 57

2.12.2. The Second Level Trigger - LVL2 _ . . . . . . . . . . . . . . . . . 57

2.12.3. Higher Trigger Levels (LVL3) and new Challenges . . . . . . . . . . 57

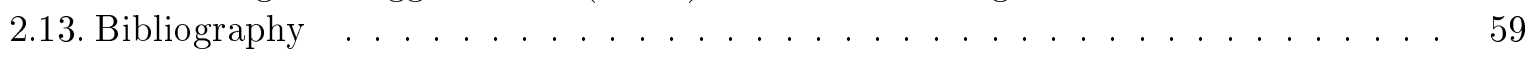

$\begin{array}{ll}\text { 3. The GE-MN System } & 63\end{array}$

3.1. GE-MN - Hardware . . . . . . . . . . . . . . . . . . 63

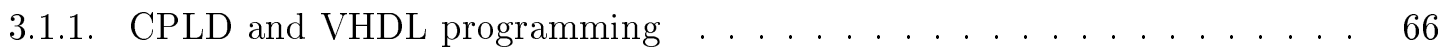

3.1.2. VMEbus Access . . . . . . . . . . . . . . . . . . 67 
3.1.3. Network Components . . . . . . . . . . . . . . . . . . . . . . 67

3.1.4. Digital Signal Processor . . . . . . . . . . . . . . . . . . . . 68

3.2. Network Communication with the GE-MN . . . . . . . . . . . . . . 68

3.2.1. Address Resolution Protocol - ARP . . . . . . . . . . . . . . . . . . . . 69

3.2.2. Internet Protocol - IP $/ \mathrm{ICMP} \ldots \ldots \ldots \ldots$

3.2.3. User Datagram Protocol - UDP . . . . . . . . . . . . . . . . . . . . 69

3.2.4. Transmission Control Protocol - TCP . . . . . . . . . . . . . . . 70

3.2.5. GE-MN Network Communication Model . . . . . . . . . . . . . . . . 70

3.2.6. Multi-Node Network Topologies with GE-MN Systems . . . . . . . . . . . 72

3.3. GE-MN - Software . . . . . . . . . . . . . . . . . . . . 73

3.4. Network Performance . . . . . . . . . . . . . . . . . . . . 75

3.4.1. Network Throughput . . . . . . . . . . . . . . . . . . . 75

3.4.2. Network Latency . . . . . . . . . . . . . . . . . . . . 76

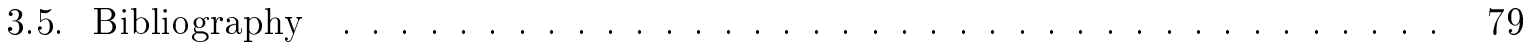

4. RICH to MDC Correlation $\quad 81$

4.1. Analysis Tools in HADES - HYDRA . . . . . . . . . . . . . . . . 82

4.1.1. Analysis Chain and used Data Set . . . . . . . . . . . . . . . 82

4.1.2. Definition of good Leptons . . . . . . . . . . . . . . . . . 83

4.2. Correlation Algorithm . . . . . . . . . . . . . . . . . . . . 84

4.2.1. Correlation Basics . . . . . . . . . . . . . . . . . 84

4.3. Implementation Details . . . . . . . . . . . . . . . . . . 85

4.3.1. Calculating the Crossed Cells - calcXed ()$\ldots \ldots \ldots$. . . . . . 86

4.3.2. Parameter Implementation in the GE-MN . . . . . . . . . . . . . . 90

4.4. Correlation Results . . . . . . . . . . . . . . . . . . . . . . . 90

4.4.1. RICH IPU Performance . . . . . . . . . . . . . . . . . . . . . . 91

4.4.2. Trigger Performance Parameters . . . . . . . . . . . . . . . . . . 91

4.4.3. Online Analysis Considerations . . . . . . . . . . . . . . . . . . . 92

4.4.4. Offline Analysis Considerations . . . . . . . . . . . . . . . . . 93

4.4.5. Correlation Analysis $-\mathrm{p}+\mathrm{p}$ at $2,2 \mathrm{GeV} \ldots \ldots \ldots \ldots$

4.4.6. Correlation Analysis $-\mathrm{C}+\mathrm{C}$ at $1,0 \mathrm{AGeV}$. . . . . . . . . . . . . 105

4.4.7. Correlation Analysis - $\mathrm{Ar}+\mathrm{KCl}$ at 1,756 AGeV . . . . . . . . . . 117

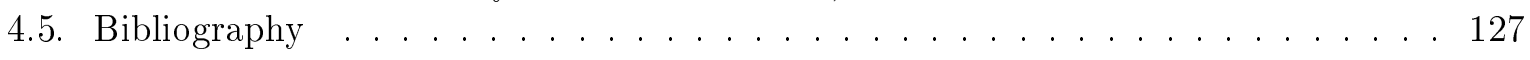

5. Summary, Discussion and Outlook 129

5.1. RICH to MDC Correlation Summary . . . . . . . . . . . . . . . . . . . 129

5.2. Discussion of the RICH to MDC Correlation Method . . . . . . . . . . . . . 130

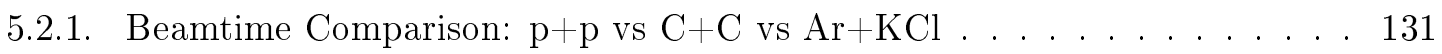

5.2.2. Outlook: RICH to MDC Correlation with other Algorithms . . . . . . . . 132

5.2.3. Outlook: Correlating the outer MDC Chambers . . . . . . . . . . . . . . 132

5.3. Discussion of the RICH to MDC Correlation Performance . . . . . . . . . . . 133

5.3.1. Performance in the GE-MN . . . . . . . . . . . . . . . . 134

5.3.2. Performance Scenarios with HADES . . . . . . . . . . . . . . 134

5.4. GE-MN Technical Summary and Outlook . . . . . . . . . . . . . . . 135

5.4.1. Directly Related Projects . . . . . . . . . . . . . . . . 136

5.4.2. The New HADES DAQ and Trigger System . . . . . . . . . . . . 136

5.4.3. RICH to MDC Correlation in the new HADES Trigger . . . . . . . . . . 139 


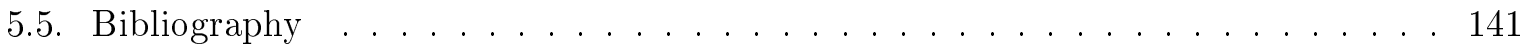

$\begin{array}{ll}\text { A. Glossary } & 143\end{array}$

$\begin{array}{lr}\text { B. HADES Collaboration Authors List } & 147\end{array}$ 


\section{Motivation and Theory Overview}

\section{Contents}

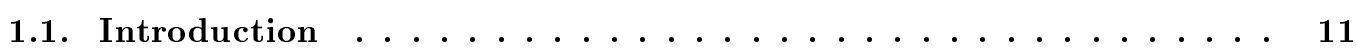

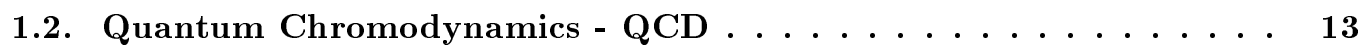

1.2.1. Asymptotic Freedom and Anti-Screening . . . . . . . . . . . . 15

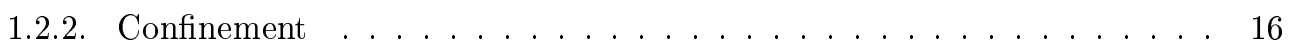

1.2.3. Symmetry and Symmetry Breaking . . . . . . . . . . . . . . 18

1.2.4. Chiral Symmetry, its Breaking and Restoration . . . . . . . . . . . 19

1.3. Subatomic Structure and Form Factors . . . . . . . . . . . 23

1.4. Light Vector Mesons and Vector Meson Dominance . . . . . . . 25

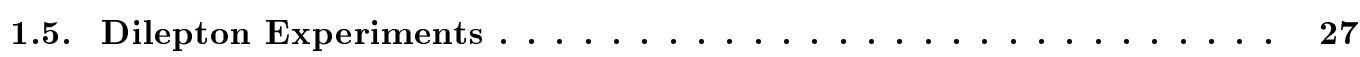

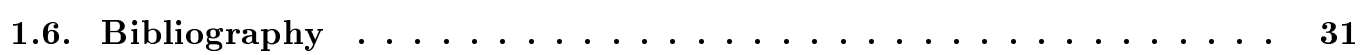

\subsection{Introduction}

The continuous research about the nature of matter, its building blocks and its interactions, in the last 100 years has led to a series of remarkable discoveries. First, the substructure of the atom was discovered - it is composed of neutrons, protons and electrons. Later, the discovery of the substructure of neutrons and protons led to the whole new world of particles composed of different quark combinations.

Four fundamental interactions, and the theories which describe them, govern the various aspects of physics: Quantum Electrodynamics (QED) describes the electromagnetic interaction which binds atoms into molecules, and thus also governs all chemistry and solid state physics. The weak interaction is explaining the radioactive $\beta$-decay as most common example. QED and the weak interaction can be unified into a common framework named the electro-weak theory. Gravity, as described by general relativity, is what creates solar systems and binds matter to planets. And finally, the strong interaction as described by the Quantum Chromodynamics (QCD) theory, which is responsible for creating all hadronic bound states, like protons and neutrons, which themselves are composed of quarks and gluons.

This thesis evolved in the context of the HADES experiment (see Chapter 2), which is designed to investigate several aspects of QCD and the properties of hadrons. In particular the properties of the $\rho, \omega$ and $\phi$ mesons are studied.

These vector mesons provide an interesting tool to investigate the properties of hadrons in the hot and dense medium of a heavy ion collision. The key property is their decay channel into lepton pairs. These interact only electromagnetically, thus they can leave the collision without further interference by strong interactions, while they still carry the properties the vector meson had at the point of its "in-medium" decay. The $\rho$ meson is especially suited for these investigations, 
since it decays inside the initial phase of a heavy ion collision. This is due to its short life time of $c \tau_{\rho} \approx 1 \mathrm{fm}$ compared to the size of a heavy ion collision, which can be as long lived as $c \tau \approx 10 \mathrm{fm}$.

The internal structure of hadrons is studied in the HADES experiment by measuring their electromagnetic form factors $F\left(q^{2}\right)$ in the time like region. One concrete example is the measurement of the $\eta$ form factor in elementary $\mathrm{p}+\mathrm{p}$ collisions. Here, also dilepton spectroscopy is used in studying the $\eta \rightarrow \mathrm{e}^{+} \mathrm{e}^{-} \gamma$ reaction .

A dilepton experiment like HADES has to cope with several challenges. First, the branching ratios into dileptons are small $\left(\approx 10^{-5}\right)$. To acquire sufficient statistics the HADES experiment features a high acceptance $(\approx 40 \%$ for dileptons) and is operated at a high interaction rate $\left(\approx 10^{6} \mathrm{~Hz}\right)$, which requires a sophisticated trigger and data acquisition system. The details of the HADES spectrometer and the data acquisition system are discussed in Chapter 2. In the search of $\mathrm{e}^{+} \mathrm{e}^{-}$-pairs from vector meson decays the main source of background are electrons from other sources. Examples are electrons from $\gamma$-conversions in the detector material as well as knock-out electrons.

The HADES spectrometer is a second generation experiment. Its predecessor experiments operating at a much higher energy are CERES [Aga05] and HELIOS [Mas95], while the DLS experiment [Por97] also operated in the HADES energy region of up to $2 \mathrm{AGeV}$. The HADES experiment features an improved momentum resolution and an improved geometrical acceptance compared to DLS.

All these experiments found an excess of dileptons at low energies in heavy ion collisions, which could not be described adequately by summing up the known sources as a cocktail. This lead to several new theoretical models to explain these results, like the dropping of the $\rho$ vector meson mass in medium [Hun97, Cas95, Li96, Bro02], or the modification of the spectral function in medium [Bra98, Rap00, Urb98, Urb00, Rap97, Cha96]. Recent results of the two current dilepton experiments HADES and NA60 are discussed in Section 1.5 together with their implications for the current models.

In the following, a selection of topics directly related to the just described physical processes are shortly reviewed from a theoretical point of view. The selection starts with an overview of the basic key aspects of the QCD theory, which also describes the properties of the light vector mesons and their strong interactions. In particular, the QCD LAGRANGIAN, asymptotic freedom and anti-screening, and the confinement of quarks in hadrons are discussed. The different types of symmetries and their consequences are discussed together with the mechanisms of explicit and spontaneous symmetry breaking. The effects and observables of the restoration of one particular symmetry, the chiral symmetry, is discussed in the framework of the linear- $\sigma$ model.

The internal structure of systems of subatomic size is accessible in scattering and annihilation processes. The relation between the observable distributions and the internal structure is discussed in terms of the form factor $F\left(q^{2}\right)$. The cross sections and thus the form factors in time like electromagnetic processes (i.g. $e^{+} e^{-}$-annihilation; DALiTz decay $\eta \rightarrow e^{+} e^{-} \gamma$ ) show an interesting behavior in the low energy transfer region. There, the photon can easily couple into a light vector meson with the same quantum numbers. This yields an enhancement around the light vector meson mass which is discussed in terms of the vector meson dominance model. This chapter concludes with a summary and discussion of the recent results of several dilepton experiments including the HADES experiment. 


\subsection{Quantum Chromodynamics - QCD}

The theory of strong interaction is called Quantum Chromodynamics (QCD). The name arises from the charge associated with the strong interaction which is called color charge. This name is chosen in analogy to the three (additive) primary colors red (R), green $(\mathrm{G})$ and blue (B) and the way they can be mixed to form the color white (in QCD: color neutral) by combining them either additively $(\mathrm{R}+\mathrm{G}+\mathrm{B}=$ white) or in $\mathrm{QCD}$ also as color anti-color (i.g. $\overline{\mathrm{R}} \mathrm{R}=$ white). One justification for this analogy is that strongly bound systems in nature have been found to be always color neutral. In the quark model, where every quark $(q)$ does carry one color charge, all known hadrons (particles made from quarks) are either mesons ( $q \bar{q}$-states) or baryons ( $q q q-$ states). The known properties of the different quarks are summarized in Table 1.1 and lead to the additional conclusion that mesons are bosons (integral spin) and baryons are fermions (spin $=n * \frac{1}{2} ; n \in \mathbb{N}$ ). The strong interaction preserves the following quantum numbers: baryon number $B$, parity $P$ and $C$-Parity $C$, and therefore also $C P$ as well as $C P T^{1}$ and the flavor quantum numbers $\mathrm{S}, \mathrm{C}, \mathrm{B}, \mathrm{T}$ (Table 1.1). The weak interaction is the relevant mechanism in processes that are violating $P, C P$, or the flavor conservation.

The grouping of the experimentally known particles, resonances and their excited states according to their quantum numbers into multiplets leads to the $S U(3)$ flavor symmetry when considering the three lightest quarks, or $S U(N)$ flavor if the $N$ lightest quarks are considered. The details are summarized in [Yao06h]. The flavor symmetry is strongly broken, as it can be seen by mass difference of the particles that belong to the same multiplet. The flavor symmetry would be perfect when all particle states belonging to the same multiplet would have identical energy states, i.e. mass.

The mediator of the strong interaction is the gluon. Its name arises from its property of being responsible for binding quarks into hadrons. Unlike the mediator of the electromagnetic force, the gluon is a carrier of the charge of its interaction, i.e. it does carry color charge. More precisely, it carries one of the eight different possible combinations of color and anti-color. This directly leads to fundamental differences in the observed phenomena compared to other interactions without this property (e.g. electromagnetic interaction): gluons directly and strongly interact with gluons.

Formally, Quantum Chromodynamics is a gauge field theory in principle similar to QED. One phenomena not observed in QED is the self interaction of the photon. In contrast, the gluons of QCD do interact strongly with one another. This ultimately leads to the interesting, but complicated anti-screening behavior, which is discussed in Section 1.2.1. The closely related phenomenon of confinement discussed in Section 1.2.2. The Lagrangian describing Quantum Chromodynamics $\mathcal{L}_{\mathrm{QCD}}$ is given in Equation (1.2). It has a more complicated structure than $\mathcal{L}_{\mathrm{QED}}$ (compare Equation (1.1) [Hal84]), since $\mathcal{L}_{\mathrm{QCD}}$ is not symmetric under local unitary $U(1)$ transformations, but under the special unitary $S U(3)$ color transformations. As usual, the EINSTEIN-convention is used, omitting the summation symbol in the formula whenever a index happens to appear twice in the same term.

\footnotetext{
${ }^{1} T$ : Time reversal
} 


$$
\begin{aligned}
\mathcal{L}_{\mathrm{QED}} & =\bar{\psi}\left(i \gamma^{\mu} D_{\mu}-m\right) \psi-\frac{1}{4} F_{\mu \nu} F^{\mu \nu} \\
D_{\mu} & =\partial_{\mu}-i e A_{\mu} \\
F_{\mu \nu} & =\partial_{\mu} A_{\nu}-\partial_{\nu} A_{\mu}
\end{aligned}
$$

Here, the four independent components of the field are given by $\psi$, while $\mathrm{m}$ is the mass of the described spin $\frac{1}{2}$ particle (i.e. the electron), $\gamma^{\mu}$ are the DIRAC matrices, $D_{\mu}$ is called the covariant derivative, $A_{\mu}$ is the vector potential and $F_{\mu \nu}$ is the field strength tensor derived from it.

Demanding $S U(3)$ local gauge invariance on a "free" Lagrangian of the form $\mathcal{L}_{0}=\bar{\Psi}_{q}\left(i \gamma^{\mu} \partial_{\mu}-\right.$ $m) \Psi_{q}$ it is possible to derive the Lagrangian of QCD [Hal84, Yao06b]:

$$
\begin{aligned}
\mathcal{L}_{\mathrm{QCD}} & =-\frac{1}{4} F_{\mu \nu}^{(a)} F_{(a)}^{\mu \nu}+i \sum_{q} \bar{\Psi}_{q}^{i} \gamma^{\mu}\left(D_{\mu}\right)_{i j} \Psi_{q}^{j}-\sum_{q} m_{q} \bar{\Psi}_{q}^{i} \Psi_{q}^{i} \\
F_{\mu \nu}^{(a)} & =\partial_{\mu} A_{\nu}^{a}-\partial_{\nu} A_{\mu}^{a}-g_{s} f_{a b c} A_{\mu}^{b} A_{\nu}^{c} \\
\left(D_{\mu}\right)_{i j} & =\delta_{i j} \partial_{\mu}+i g_{s} \sum \frac{1}{2} \lambda_{i, j}^{a} A_{\mu}^{a}
\end{aligned}
$$

The variable $\Psi_{q}^{i}$ represents the four component DIRAC spinor of color $i$ and quark flavor $q$. $F_{\mu \nu}^{(a)}$ is again the field strength tensor as well as $D_{\mu}$ is again the covariant derivative, but both now feature additional terms representing the more elaborate $S U(3)$ group structure. The $f_{a b c}$ are the structure constants of the $S U(3)$ algebra and the $3 \times 3$ matrices $\lambda^{a}$ are the generators of the $S U(3)$ transformations. Furthermore, $g_{s}$ is the QCD coupling constant and $m_{q}$ is the mass of the quark of flavor $q$. The color index runs from 1 to 8 as given by the group structure $\left(N^{2}-1=3^{2}-1=8\right)$, while the flavor index $q$ runs over the six quark flavors $u, d, s, c, b, t$ as given in Table 1.1. 


\begin{tabular}{|c|c||c|c|c|c|c|c|c|c|}
\hline Symbol & Name & Bare Quark Mass & $I\left(J^{P}\right)$ & Charge & $I_{Z}$ & $\mathrm{~S}$ & $\mathrm{C}$ & $\mathrm{B}$ & $\mathrm{T}$ \\
\hline \hline$u$ & up & $1,5 \mathrm{MeV}$ to $3,0 \mathrm{MeV}$ & $\frac{1}{2}\left(\frac{1}{2}^{+}\right)$ & $+\frac{2}{3} e$ & $+\frac{1}{2}$ & 0 & 0 & 0 & 0 \\
\hline$d$ & down & $3 \mathrm{MeV}$ to $7 \mathrm{MeV}$ & $\frac{1}{2}^{2}\left(\frac{1}{2}^{+}\right)$ & $-\frac{1}{3} e$ & $-\frac{1}{2}$ & 0 & 0 & 0 & 0 \\
\hline$s$ & strange & $(95 \pm 25) \mathrm{MeV}$ & $0\left(\frac{1}{2}^{+}\right)$ & $-\frac{1}{3} e$ & 0 & -1 & 0 & 0 & 0 \\
\hline$c$ & charm & $(1,25 \pm 0,09) \mathrm{GeV}$ & $0\left(_{\frac{1}{2}}^{+}\right)$ & $+\frac{2}{3} e$ & 0 & 0 & +1 & 0 & 0 \\
\hline$b$ & bottom & $(4,2 \pm 0,07) \mathrm{GeV}$ & $\left.0 \frac{1}{2}^{+}\right)$ & $-\frac{1}{3} e$ & 0 & 0 & 0 & -1 & 0 \\
\hline$t$ & top & $(174,2 \pm 3,3) \mathrm{GeV}$ & $0\left(\frac{1}{2}^{+}\right)$ & $+\frac{2}{3} e$ & 0 & 0 & 0 & 0 & +1 \\
\hline
\end{tabular}

Table 1.1.: Quark Properties Summary [Yao06a].

Quarks have Baryon number $B=+1 / 3$, anti-quarks $B=-1 / 3$. The other variables are: $I-$ isospin, $I_{z}-z$ component of the isospin, $J$ - spin, $P$ - parity, $e$ - electron charge. The flavor quantum numbers are $\mathrm{S}$ - strangeness, $\mathrm{C}$ - charmness, B - bottomness, $\mathrm{T}$ - topness. The bare quark masses are still under active discussion, a detailed discussion can be found in the references in [Yao06a].

\subsubsection{Asymptotic Freedom and Anti-Screening}

In Quantum Electrodynamic the effect of charge screening leads to a reduction of the actual visible amount of charge at large distances (low probe energy). The strength of the electromagnetic interaction, as characterized by $\alpha$, is the asymptotic value at large interaction distances. The actual screening effect is caused by virtual $\gamma^{*}$-quanta emitted by a charge, in this example an electron, which couple to $e^{+} e^{-}$-pairs. Since the positrons are attracted by the real charge, the electron surrounds itself with a positron "cloud". Thus, the effective charge, as seen from outside the cloud, is reduced. A probe of sufficient energy penetrates the surrounding $e^{+} e^{-}$-cloud and sees with decreasing distance an increasing amount of charge, i.e. it sees the coupling "constant" $\alpha(r)$ is rising with decreasing distance. An example of an experimental confirmation is discussed in [Ach05].

In QCD a similar but reversed effect is observed. Virtual gluons emitted by the real color charge couple, in addition to coupling into $\bar{q} q$-pairs in analogy to QED, also into gluon loops. The self interactions of this virtual gluon cloud lead to anti-screening and asymptotic freedom (derivation see [Hal84]). Anti screening describes the opposite effect of screening: with increasing distance the strength of the interaction $\alpha_{\mathrm{S}}(r)$ is strongly rising, with $\alpha_{\mathrm{S}}(r) \approx 1$ at a distance of $r=1 \mathrm{fm}$ (confinement barrier). At the same time the color field strength is largely reduced at small distances (or large momentum transfer), and two close quarks asymptotically approach a state of being free, non interacting particles. The nominal value of $\alpha_{\mathrm{S}}$ at the mass of the $Z^{0}$ boson is given by $\alpha_{S}\left(m_{Z}\right)=0,1176 \pm 0,0020$ [Yao06c], while a functional description of the running coupling can be found in [Yao06b]. This effect in terms of the momentum transfer $Q$ is shown in Figure 1.1 as it is expected by theory and actually seen by different experiments. 


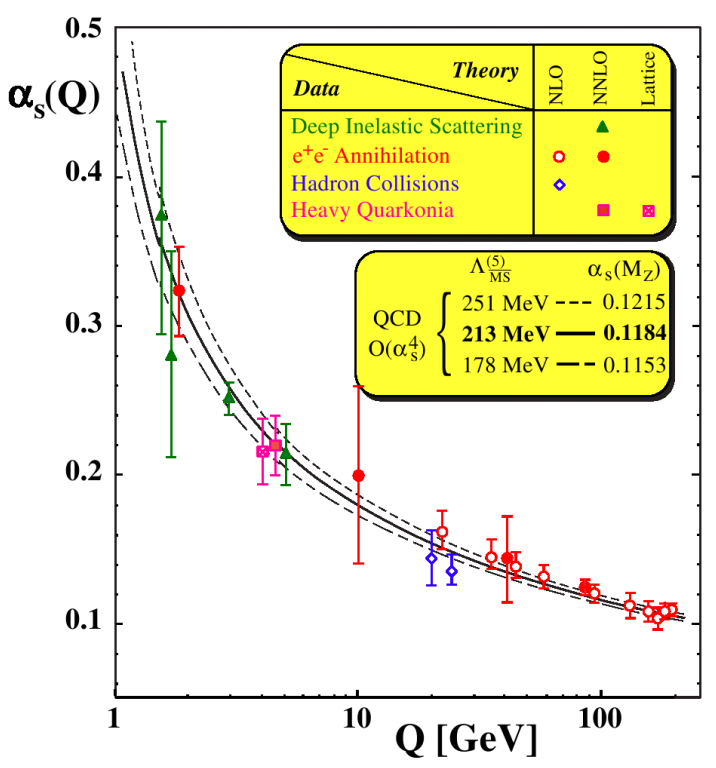

Figure 1.1.: Strong Coupling "Constant" $\alpha_{\mathbf{S}}(Q)$ by Momentum Transfer [Tas01].

With increasing momentum transfer $Q$ the distance where the interaction takes place is reduced and the coupling constant $\alpha_{\mathrm{S}}(Q)$ drops. The sources of the data points and their error bars are

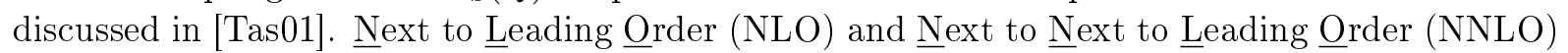
calculation are compared to Lattice QCD calculations.

\subsubsection{Confinement}

The experimental fact that all observed objects are color neutral, and thus no free single quarks are observed, is referred to as confinement. A simple picture of confinement is sketched in Figure 1.2. The force between two quarks can be phenomenologically modeled by an ansatz of a potential with two terms in the relative distance $r-$ an $1 / r$ term and a term linear in $r$ :

$$
V(r) \approx-\frac{4}{3} \cdot \alpha_{\mathrm{S}}(r) \cdot \hbar c \cdot \frac{1}{r}+k \cdot r
$$

The first term models a Coulomb like potential at short distances, while the linear term ensures confinement. The variable $\alpha_{\mathrm{S}}(r)$ is the distance dependent strong coupling constant discussed in Section 1.2.1. The magnitude of the string tension $k$ is of the order of $k=1 \mathrm{GeV} / \mathrm{fm}$, which ensures that already at very small separations of quarks from one another (FERMI scale) the binding energy is high enough to create a new $q \bar{q}$-pair in the center of the string, breaking it as depicted in Figure 1.2 into two color neutral particles. The formation of back-to-back particle pairs observed as back-to-back jets in the final state in high energy collider experiments is a nice example of how this string breaking can be observed. Lattice QCD calculations support this picture quantitatively (compare Figure 1.3) and also show flux-tubes for systems of three quarks, as for example in [Ich03]. 


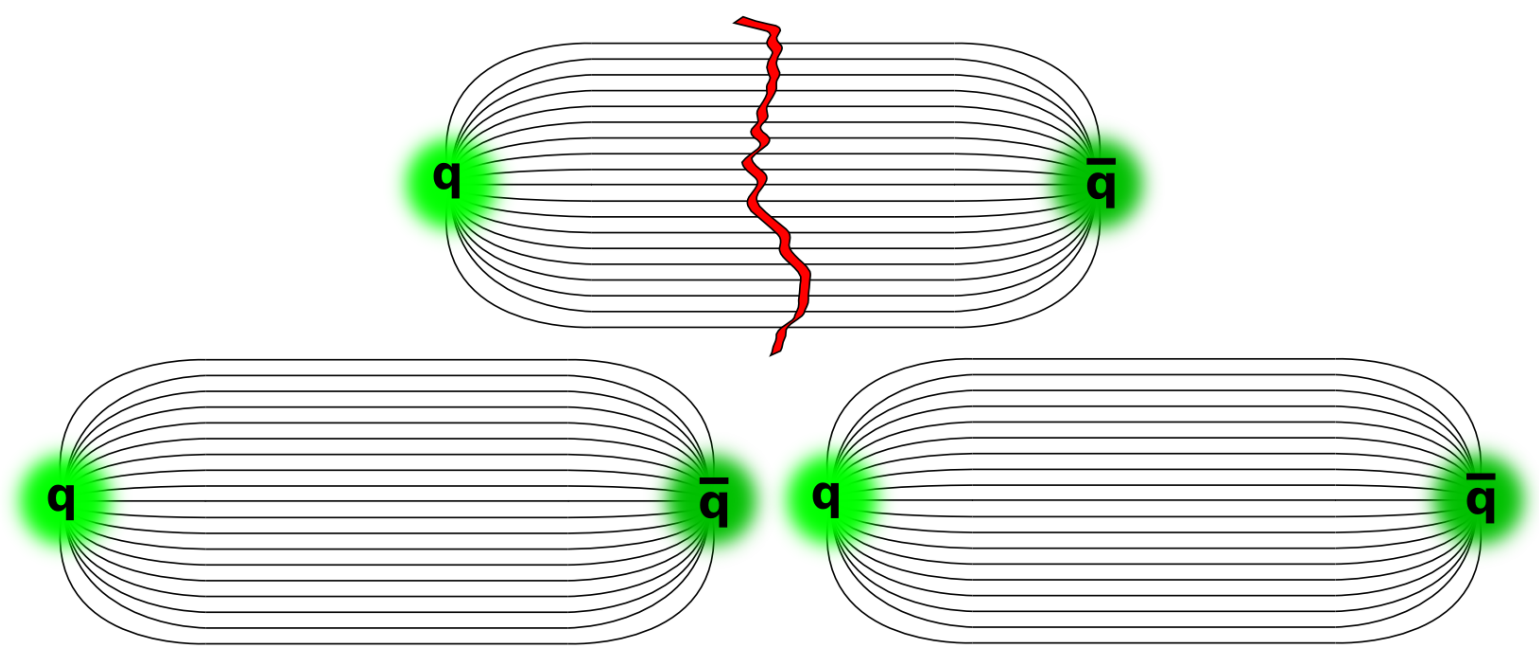

Figure 1.2.: Schematic Picture of the Color Field and the String Breaking.

The $k \cdot r$ term in the potential Equation (1.3) leads to the depicted stretched field lines that form a "flux-tube" with a string tension $k$. It should be noted how different this picture is compared to the field of two electric charges. When the two quarks are being separated, the energy of the binding increases according to the $k \cdot r$ term. Due to the high coupling strength $k=1 \mathrm{GeV} / \mathrm{fm}$ the energy is already at very small separations sufficient to create a new $\bar{q} q$-pair in between, and thus the string breaks. Light Green and dark Green represent an arbitrary color anti-color

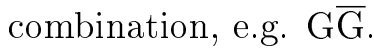

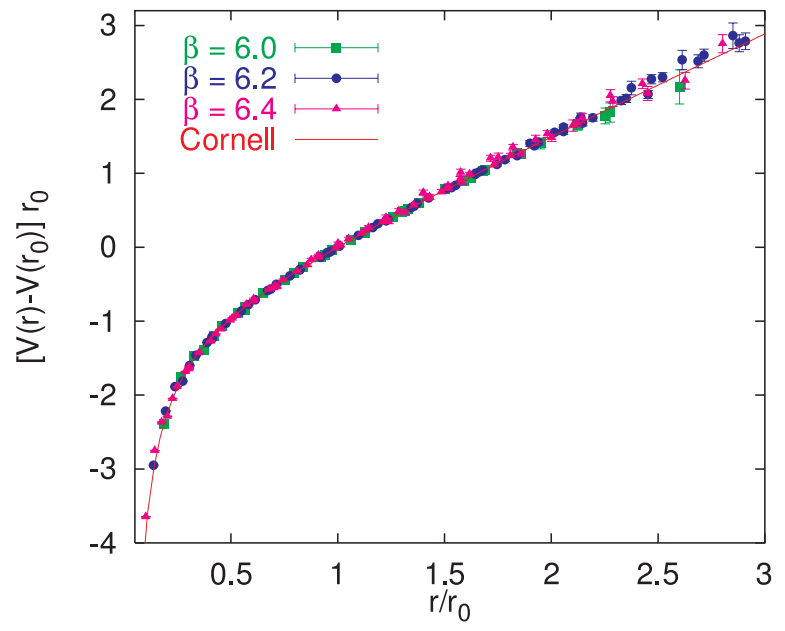

(a) Quenched Wilson Action Potential

Figure 1.3.: Normalized Quenched Wilson Action $S U(3)$ Potential [Bal00].

This figure shows the QCD potential as it is derived from quenched lattice simulation. The agreement to Equation (1.3) should be noted and all further details are found in [Bal00]. 


\subsubsection{Symmetry and Symmetry Breaking}

Symmetries are of fundamental interest for physics. The NoETHER theorem [Noe18] connects symmetries directly with the appearance of a conserved quantity. Classical examples are that the invariance under translations, time displacements and rotations lead respectively to the conservation of momentum, energy and angular momentum. In field theory additional symmetries are of interest, especially local gauge symmetries. In particular, the symmetries under the transformations of the unitary $U(1)$ and special unitary $S U(N)$ groups are relevant. For example, the local $U(1)$ symmetry of QED enforces the conservation of the electric charge.

As interesting as the symmetries themselves is the special case when a symmetry is "broken". A symmetry can be broken explicitly by adding a term which does not obey the symmetry to an otherwise symmetrical Lagrangian (e.g. in QED a photon mass term). Spontaneous symmetry breaking, on the other hand, is realized if a symmetry of the Lagrangian is hidden by a particular choice of the ground state of the potential, which does not show the symmetry anymore. A famous example is the so called Mexican hat potential as sketched in Figure 1.4(b). This potential is perfectly rotational symmetric, but the point in the center is not a stable minimum, so the ground state a real system chooses is somewhere on the minimum ring. When this happens rotations obviously do change something in the system, so the symmetry is spontaneously broken. Both types of symmetry breaking have important practical physical implications.

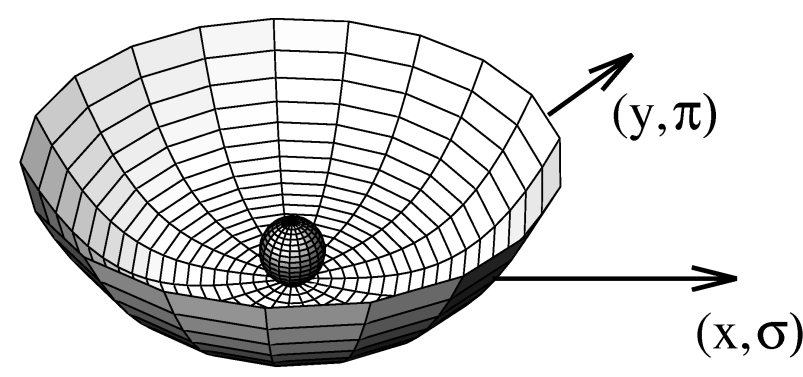

(a) Ground State Symmetric

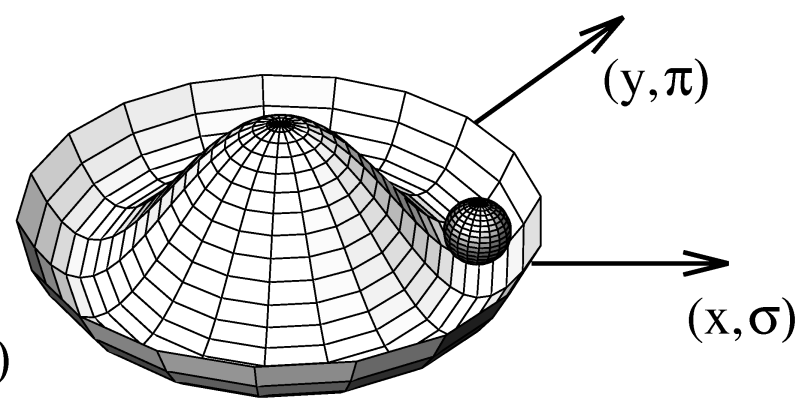

(b) Ground State not Symmetric

Figure 1.4.: Mexican hat potential in 3D [Koc02].

This potential is illustrating how the random choice of a ground state in a fully rotational symmetric potential breaks, or better hides, the symmetry of the potential, as it is demonstrated in (b). The axis $x$ and $y$ are also labeled as $\sigma$ and $\pi$ for the explanation of the $\sigma$ and $\pi$ fields as discussed in detail in Section 1.2.4 and Figure 1.5.

The explicit symmetry breaking term can have a certain strength in relation to the other terms in the Lagrangian, and if it is small enough, the physical system will still show characteristics of the symmetry, although it is in principle broken. This can make it possible to neglect the symmetry breaking term and describe the system as approximately symmetric, as long as this term is small enough. It should be noted that the different contributions to the Lagrangian can depend on different energy scales, which provides a way to virtually restore a symmetry by changing the energy scale under discussion, if the symmetry breaking term is not (or much weaker) increasing with energy. 
Spontaneous symmetry breaking is conceptually more involved and will be sketched sticking to the Mexican hat example from Figure 1.4. The GoLDSTONE theorem states that whenever a continuous symmetry is spontaneously broken a new mass-less scalar particle appears, i.e. the Goldstone boson. In the Mexican hat picture this corresponds to excitations on the minimum ring around the symmetry of the potential (azimuthal excitations).

\subsubsection{Chiral Symmetry, its Breaking and Restoration}

With the chirality operator $\gamma^{5}=i \gamma^{0} \gamma^{1} \gamma^{2} \gamma^{3}$ (DIRAC matrices [Hal84]) it is possible to define the right and left handed projection operators $P_{R}$ and $P_{L}$ :

$$
P_{R}=\frac{1}{2}\left(1+\gamma^{5}\right), \quad P_{L}=\frac{1}{2}\left(1-\gamma^{5}\right)
$$

For massive fermions the projections of $P_{R / L}$, called the handedness, are not good quantum numbers, but in the limit of vanishing masses $m=0$ in Equation (1.2) this Lagrangian acquires a symmetry that is connected to the conservation of the handedness of quarks. In other words, the so called chiral symmetry of the interactions is $S U(2)_{R} \times S U(2)_{L}$ flavor for a model treating only up and down quarks. This decouples the left handed world from the right handed one. Equivalently the Lagrangian is symmetric under the axial-vector transformation $\Lambda_{A}$ and the vector transformation $\Lambda_{V}$ (group structure: $\left.S U(2)_{V} \times S U(2)_{A}\right)$ [Koc02, Koc95]:

$$
\begin{aligned}
\Lambda_{V}: \quad \psi \rightarrow e^{-i \frac{\tau}{2} \vec{\Theta}} \psi \approx\left(1-i \overrightarrow{\frac{\tau}{2} \vec{\Theta}}\right) \psi \\
V_{\mu}^{a}=\bar{\psi} \gamma_{\mu} \frac{\tau^{a}}{2} \psi \\
\Lambda_{A}: \quad \psi \rightarrow e^{-i \gamma^{5} \frac{\vec{\tau}}{2} \vec{\Theta}} \psi \approx\left(1-i \gamma^{5} \frac{\tau}{2} \vec{\Theta}\right) \psi \\
A_{\mu}^{a}=\bar{\psi} \gamma_{\mu} \gamma^{5} \frac{\tau^{a}}{2} \psi
\end{aligned}
$$

Here $\vec{\tau}$ refers to the PAULI (iso-)spin matrices, $V_{\mu}^{a}$ and $A_{\mu}^{a}$ are the conserved currents associated with the vector and axial vector symmetries, while $\vec{\Theta}$ gives the parameters of the transformation.

The vector transformation $\Lambda_{V}$ can be identified with isospin rotations and it is known to be conserved in the strong interaction. In contrast, the axial-vector transformation $\Lambda_{A}$ is known to be heavily broken, since the axial-vector symmetry rotations can transform a $\rho$-particle into a $a_{1}$-particle. Thus, these two should have the same energy eigenvalue, i.e. the same mass. This is obviously not the case since there is a large gap in the masses (compare Table 1.2 on page 27).

If the energy scale involved in hadron physics $\left(\Lambda_{Q C D} \approx 200 \mathrm{MeV}\right)$ is compared to the much lighter up and down quark masses (compare Table 1.1), one can assume by following the arguments from Section 1.2.3 that the axial current should be at least approximately (or partially) conserved. This is called the Partially $\underline{\text { Conserved }}$ Axial $\underline{\text { Current }}$ (PCAC) hypothesis. The difference in mass of the $\rho$ and $a_{1}$ is too large to be explained exclusively by the small quark masses, instead it is explained by the spontaneous breaking of chiral symmetry. 

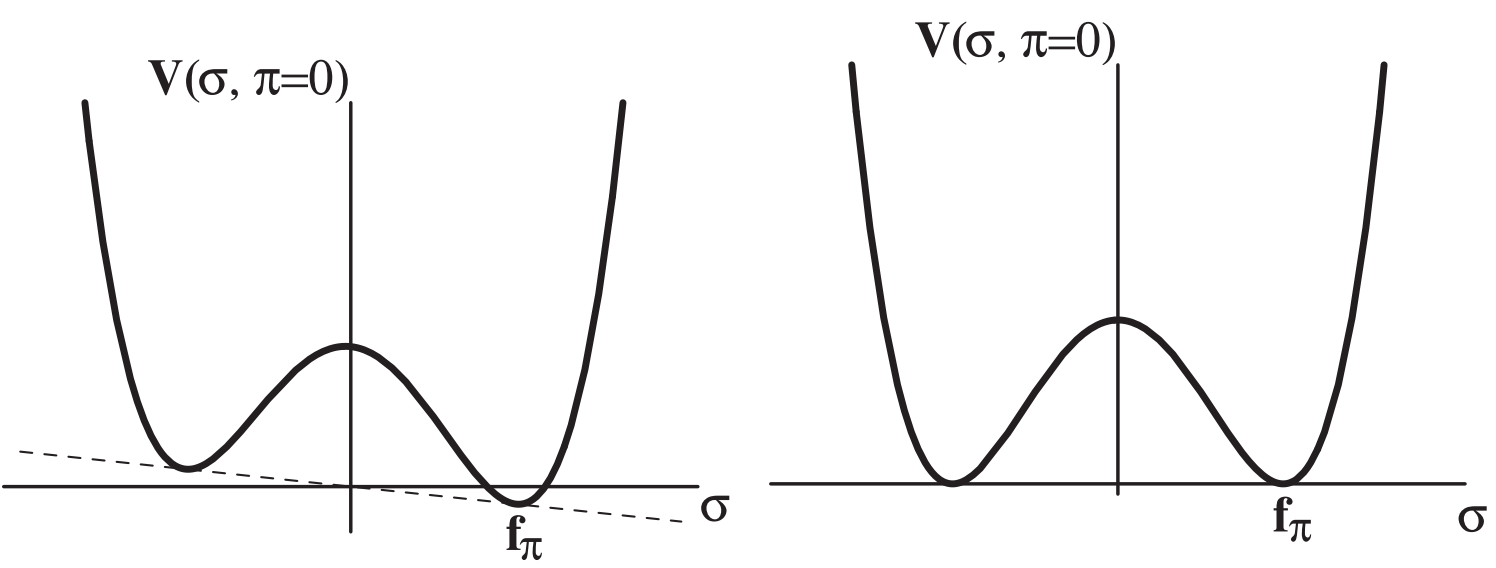

Figure 1.5.: Linear Sigma Model Mexican Hat Potential as 2D cut [Koc02].

The figure on the right shows the potential of the linear sigma model without an explicitly symmetry breaking term, in contrast to the figure on the left which shows the tilt of the Mexican hat that grants the pion its mass within this model.

The linear sigma model uses the Mexican hat potential shown in Figure 1.5 as a two dimensional cut of Figure 1.4. The shape ensures a spontaneous symmetry breaking because the ground state does not follow the rotational symmetry anymore. In this picture it is the massive $\sigma$ state (new PDG name: $f_{0}$ [Yao06d] compare Table 1.2), which, in the quark picture, has a finite vacuum expectation value $\langle\bar{q} \mid q\rangle \neq 0$, since it has the quantum numbers of the vacuum. The $\sigma$ excitations correspond to shifts along the $\sigma$ axis, while the excitations of the $\pi$ in this picture correspond to small mass-less excitations in the valley along the $\pi$ axis, i.e. the pions are the Goldstone bosons. It is well known from experiment that the pion mass is not zero, but remarkably small compared to the mass of all the other hadrons. The linear sigma model includes an explicit symmetry breaking term given by the mass of the quarks to describe this fact. The resulting tilted Mexican hat potential is shown in Figure 1.5 (a) and ensures that pion excitations are not mass-less anymore, while ensuring that their mass will be small, as long as the explicit symmetry breaking term is also small compared to the scale of the spontaneous symmetry breaking.

A measure of the strength of the symmetry breaking is the finite quark expectation value

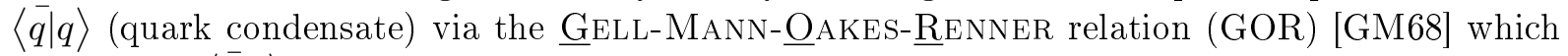
also relates $\langle\bar{q} \mid q\rangle$ to measurable quantities:

$$
m_{\pi}^{2} f_{\pi}^{2}=-\frac{1}{2}\left(m_{u}+m_{d}\right) \cdot\langle 0|\bar{u} u+\bar{d} d| 0\rangle
$$

Here $m_{\pi}$ denotes the pion mass, $f_{\pi}$ is the pion decay constant, $m_{u}$ and $m_{d}$ are the up and down quark masses. 
In addition, it can be shown in a chiral perturbation model [Koc02], that the expectation value of the quark condensate has a dependency on the temperature $T$ and the actual nuclear density $\rho$ in relation to the normal nuclear density $\rho_{0}$. These "in-medium" effects are described in first order by [Ger89, Koc02]:

$$
\begin{aligned}
\langle\bar{q} q\rangle_{T} & =\langle 0|\bar{q} q| 0\rangle \cdot\left(1-\frac{T^{2}}{8 f_{\pi}^{2}}\right) \\
\langle\bar{q} q\rangle_{\rho} & =\langle 0|\bar{q} q| 0\rangle \cdot\left(1-0,35 \cdot \frac{\rho}{\rho_{0}}\right)
\end{aligned}
$$

Thus the temperature dependency is quadratic, while the density dependence is linear and has a strength of already $35 \%$ at normal nuclear density. It should be noted that Equation (1.6) is only valid for small values of $\rho$. A diagram visualizing the combined effect of nonzero density and temperature, as well as some experimentally accessible regions, is shown in Figure 1.6. At high temperature and/or density the quark condensate drops to zero, and since it is a measure of the strength of the symmetry breaking, the symmetry breaking vanishes. At this so called chirally restored phase the discussed effects of the symmetry breaking also vanish.

The inspection of the QCD phase diagram, as shown in Figure 1.7, shows where the chirally restored phase is expected, if it exists at all and is not hidden by the overlap of a deconfined phase, where the individual quarks are no longer confined into color neutral hadrons, but form a quark gluon plasma, which is also expected at high temperatures and densities.

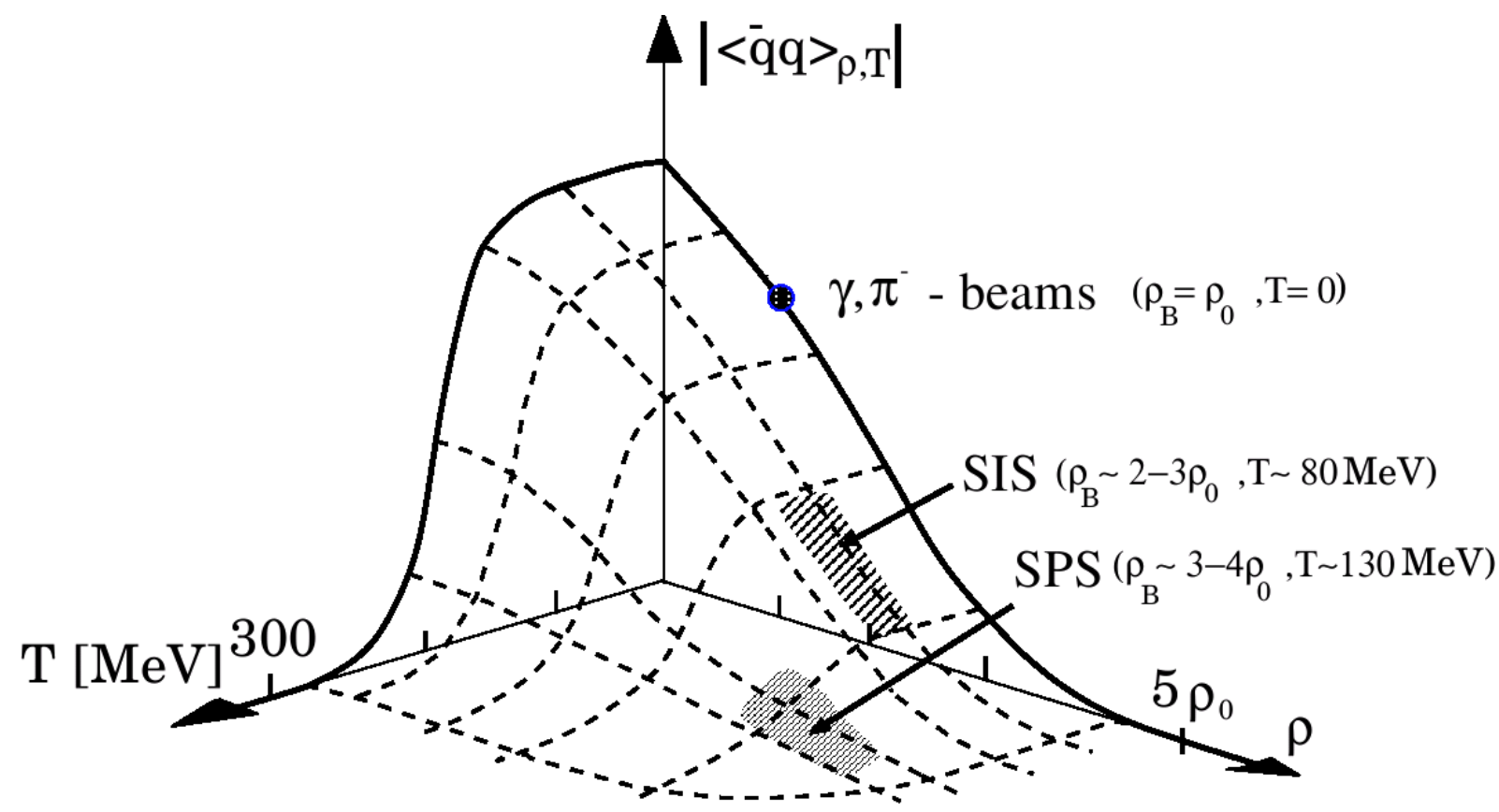

Figure 1.6.: Dependency of the Quark Condensate on Temperature and Density [Kli90].

At high temperature or density $\langle\bar{q} q\rangle_{T, \rho}$ drops to zero and a chirally restored phase is expected.

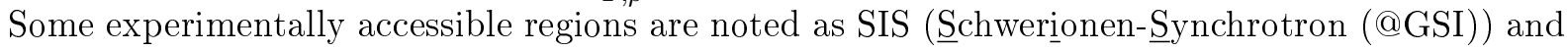
SPS (․uper Proton Synchrotron (@CERN)) 


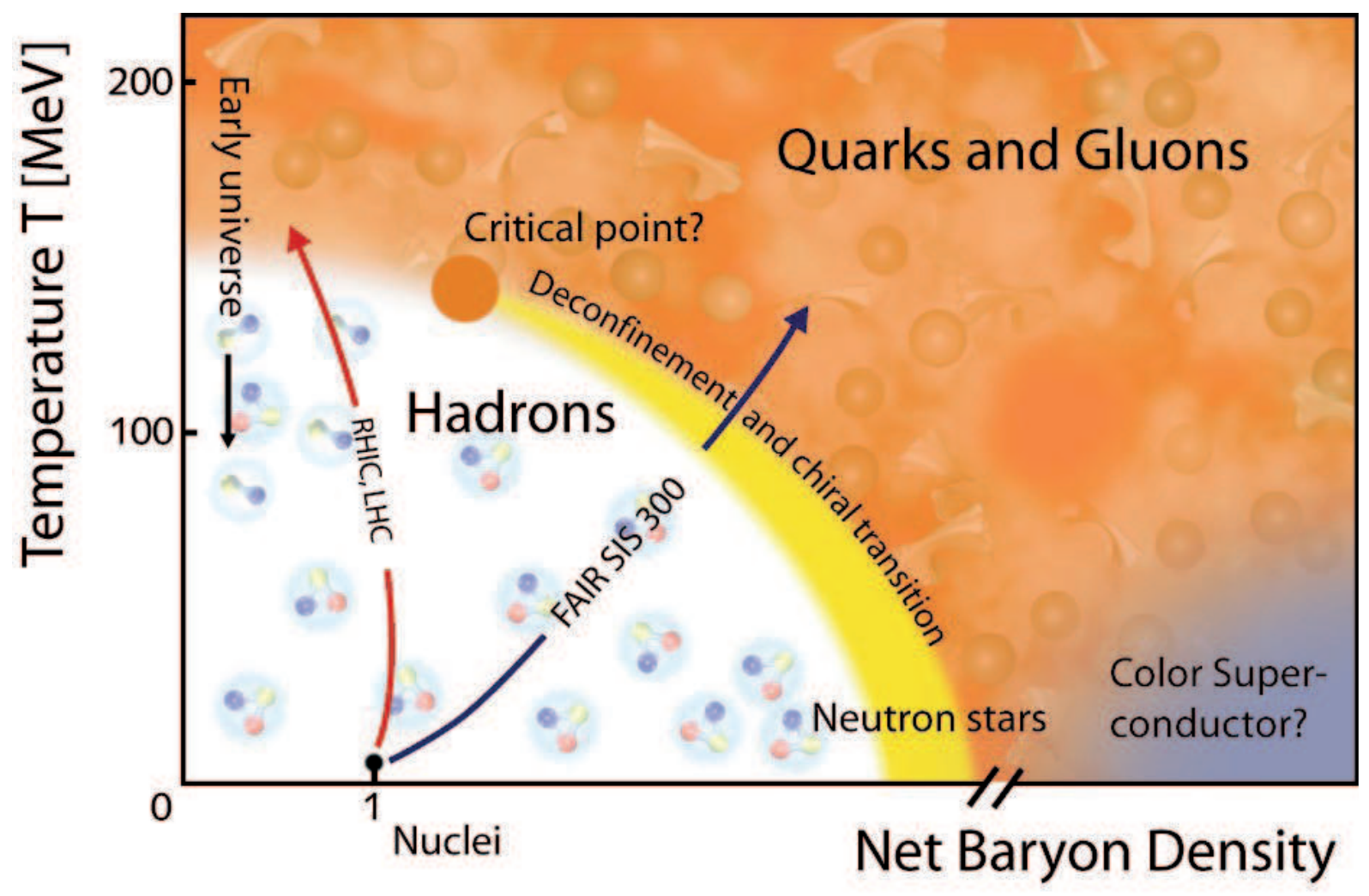

Figure 1.7.: QCD Phase Diagram [Gut06].

This figure shows a plot of temperature versus the Net Baryon Density $(N B D)$ of strongly interacting systems. Some regions of the QCD phase diagram are indicated, which are accessible at different accelerator facilities. In addition, the areas that are present as condensed (atomic) nuclear matter $(T \approx 0, N B D=1)$ are shown, as well as those which are expected to be present as neutron stars. A phase transition separating the world of hadrons as we see it, and a deconfined phase in which quarks and gluons exist as free particles in a quark-gluon plasma is sketched, including a possible critical point. For more a quantitative review consult [Cle98] and the references therein. 


\subsection{Subatomic Structure and Form Factors}

The internal structure of an object can be investigated by shooting projectiles at it and measuring the resulting scattering distributions. A detailed analysis (as found in [Pov06, Hal84]) leads to several formulas for the differential cross sections $\mathrm{d} \sigma / \mathrm{d} \Omega$. The description of the scattering depends on the properties of projectile and target. In the simplest case of classical point-like particles (both projectile and target) without spin the elastic scattering process without recoil is described by the RUTHERFORD differential cross section (cross section $\sigma$ per solid angle $\Omega$ ) of a projectile with kinetic energy $E_{k i n}$ and electric charge $z e$ on a point like target with charge $Z e$ :

$$
\begin{aligned}
& \left(\frac{\mathrm{d} \sigma}{\mathrm{d} \Omega}\right)_{\text {Rutherford }}^{\text {classical }}=\frac{\left(z Z e^{2}\right)^{2}}{\left(4 \pi \epsilon_{0}\right)^{2} \cdot\left(4 E_{k i n}\right)^{2} \sin ^{4} \frac{\theta}{2}} \\
& \left(\frac{\mathrm{d} \sigma}{\mathrm{d} \Omega}\right)_{\text {Rutherford }}^{\text {relativistic }}=\frac{4 Z^{2} \alpha^{2}(\hbar c)^{2} \cdot E^{\prime 2}}{|\vec{q} c|^{4}}=\frac{Z^{2} \alpha^{2}(\hbar c)^{2}}{4 \cdot E^{2} \sin ^{4} \frac{\theta}{2}}
\end{aligned}
$$

The variable $\epsilon_{0}$ is the permittivity of free space, $\theta$ the scattering angle, $\alpha$ is the fine structure constant and $\hbar$ is the PLANK-constant. The variable $E^{\prime}$ ( $=E$ if no recoil) gives the energy in the final state, and $\vec{q}$ is the momentum transfer in the process.

A more elaborate formula, called the MoTT cross section, describes the scattering of electrons $(\operatorname{spin} 1 / 2)$ :

$$
\begin{aligned}
& \left(\frac{\mathrm{d} \sigma}{\mathrm{d} \Omega}\right)_{\text {Mотт }}^{\text {point }}=\left(\frac{\mathrm{d} \sigma}{\mathrm{d} \Omega}\right)_{\text {Mott }}^{*} \cdot \frac{E^{\prime}}{E} \\
& \left(\frac{\mathrm{d} \sigma}{\mathrm{d} \Omega}\right)_{\text {Motт }}^{*}=\left(\frac{\mathrm{d} \sigma}{\mathrm{d} \Omega}\right)_{\text {Rutherford }} \cdot\left(1-\beta^{2} \sin ^{2}\left(\frac{\theta}{2}\right)\right)
\end{aligned}
$$

The variable $\beta=v / c$ is the relativistic velocity, $\theta$ is the scattering angle, the initial state energy is denoted by $E$, so $\frac{E^{\prime}}{E}$ is the recoil factor and the star indicates that recoil effects are not included in the cross section.

To investigate the internal charge distribution inside a nucleus the scattering of electrons with sufficient energy is an option. The comparison of the measured differential cross section with the cross section of a point like target yields the form factor $F\left(q^{2}\right)$ :

$$
\left(\frac{\mathrm{d} \sigma}{\mathrm{d} \Omega}\right)=\left(\frac{\mathrm{d} \sigma}{\mathrm{d} \Omega}\right)_{\text {Мотт }}^{\text {point }} \cdot\left|F\left(q^{2}\right)\right|^{2}
$$

In the non relativistic limit, the form factor $F\left(q^{2}\right)$ can be interpreted as the FouRIER transform of the charge distribution in the target. This is in complete analogy to classical optics, where the FouRIER transform of the spatial extents of an illuminated object describes the diffraction of light on the object, and thus carries the complete information about the shape of the object. 


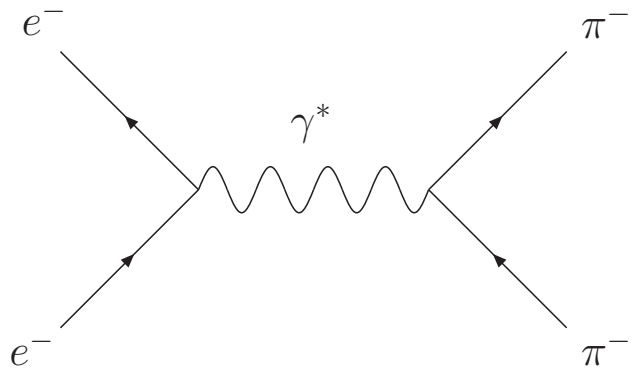

(a)

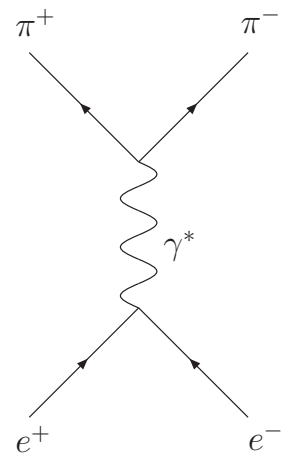

(b)

Figure 1.8.: Feynman Diagrams of Electron Scattering and Annihilation.

Figure a) shows $e^{-} \pi^{-}$scattering (space-like, $q^{2}<0$ ). Reversing the diagram leads to the $e^{+} e^{-}$annihilation and $\pi^{+} \pi^{-}$pair production diagram (time-like, $\left.q^{2}>0\right)$ shown in $\mathrm{b}$ ). The time axis is pointing upwards in both figures.

To study the structure of nucleons it has to be considered that the constituents of the nucleon also have spin and the distributions of its magnetic and electric moments are not equal. The solution is to refine the MotT formula into the Rosenbluth formula [Ros50]:

$$
\left(\frac{\mathrm{d} \sigma}{\mathrm{d} \Omega}\right)_{\text {Rosenbluth }}=\left(\frac{\mathrm{d} \sigma}{\mathrm{d} \Omega}\right)_{\text {Mott }}^{\text {point }} \cdot\left[\frac{G_{E}^{2}\left(Q^{2}\right)+\tau G_{M}^{2}\left(Q^{2}\right)}{1+\tau}+2 \tau G_{M}^{2}\left(Q^{2}\right) \tan ^{2}\left(\frac{\theta}{2}\right)\right]
$$

The variables which enter in addition are the electric form factor $G_{E}^{2}\left(Q^{2}\right)$, the magnetic form factor $G_{M}^{2}\left(Q^{2}\right)$, the 4-momentum transfer $-Q^{2}=q^{2}=\left(\frac{E}{c}\right)^{2}-p^{2}$ and the abbreviation $\tau=\frac{Q^{2}}{4 M^{2} c^{2}}$, where $M$ is the mass of the target particle. It should be noted that in the case of relativistic particles the form-factors are no longer directly the FOURIER-transform of the spatial distributions, but nevertheless still carry the full spatial information.

The Rosenbluth formula can be used to investigate the structure of a nucleon by first measuring the scattering cross section for different fixed values of $Q^{2}$ at different scattering angles $\theta$. The analysis of this data in terms of the Rosenbluth formula yields the form factors $G_{E}^{2}\left(Q^{2}\right)$ and $G_{M}^{2}\left(Q^{2}\right)$, which lead (when investigating their $Q^{2}$ dependence) to the spatial distribution of charges and magnetic moments in the nucleus.

The numerical value of $q^{2}$ is negative ("space-like" region) in scattering processes since no energy and only 3-momentum is transferred between projectile and target. The "time-like" region of $q^{2}>0$ becomes accessible in annihilation processes where the 3-momentum transfer is zero and only energy is transferred. Apart from the "time-like" region of 4-momenta $q^{2}<\left(2 \times M_{\text {particle }}\right)$ where not enough energy is available to produce the particle anti-particle pair of interest in the outgoing channel, the whole $q^{2}$ region can be investigated. Example FEYnMAN-diagrams of these processes are shown in Figure 1.8. 


\subsection{Light Vector Mesons and Vector Meson Dominance}

The cross sections (and thus also the forms factor involved) in time-like electromagnetic processes (i.g. $e^{+} e^{-}$-annihilation, DALITZ decay $\eta \rightarrow e^{+} e^{-} \gamma$ ) in the low energy transfer region $\left(q^{2} \lesssim 1(\mathrm{GeV} / c)^{2}\right)$ show some interesting characteristics: a strong enhancement in the mass range of light vector mesons $(\rho / \omega)$ as shown as an example in Figure 1.9. This can be described in the framework of the Vector Meson Dominance (VMD) model (as suggested by [Sak69]), where the electromagnetic coupling of leptons to hadrons at low energy is described by assuming that the coupling is also mediated by a vector meson. The quantum numbers a virtual photon possess $\left(J^{P C}\right)=\left(1^{--}\right)$allow it to directly couple into a quark anti-quark $(q \bar{q})$ state, i.e. a vector meson with the same quantum numbers. In Table 1.2 the characteristics of the vector mesons with sufficiently low mass can be found. The VMD model explains the hadronic structure of the coupling of the virtual photon at low energies, and the FEYNMAN diagrams describing this behavior can be found in Figure 1.10. More formally the earlier presented peak in the pion form factor is described well by

$$
F_{\pi}\left(q^{2}\right)=\frac{m_{\rho}^{2}}{m_{\rho}^{2}-q^{2}-\mathrm{i} \cdot m_{\rho} \Gamma_{\rho}\left(q^{2}\right)}
$$

where $m_{\rho}$ is the mass of the $\rho$-meson and $\Gamma_{\rho}$ is the $\rho \rightarrow \pi \pi$ decay width. More general, the dependence of the form factor on the mass of the vector meson $\left(M_{\mathrm{VM}}\right)$ according to VMD is given by:

$$
F\left(q^{2}\right)=\frac{M_{\mathrm{VM}}^{2}}{M_{\mathrm{VM}}^{2}-q^{2}}
$$

The observed sudden and sharp drop in $\left|F_{\pi}\left(q^{2}\right)\right|^{2}$ in Figure 1.9 around $780 \mathrm{MeV}$ at the mass of the $\omega(782)$ (compare Table 1.2) can be understood by the interference of $\rho$ and $\omega$. This effect is known as $\rho-\omega$ mixing.

A more recent review of the topic in terms of the hadronic structure function $F_{2}^{\gamma}$ can be found in [Yao06e, Nis00]. 

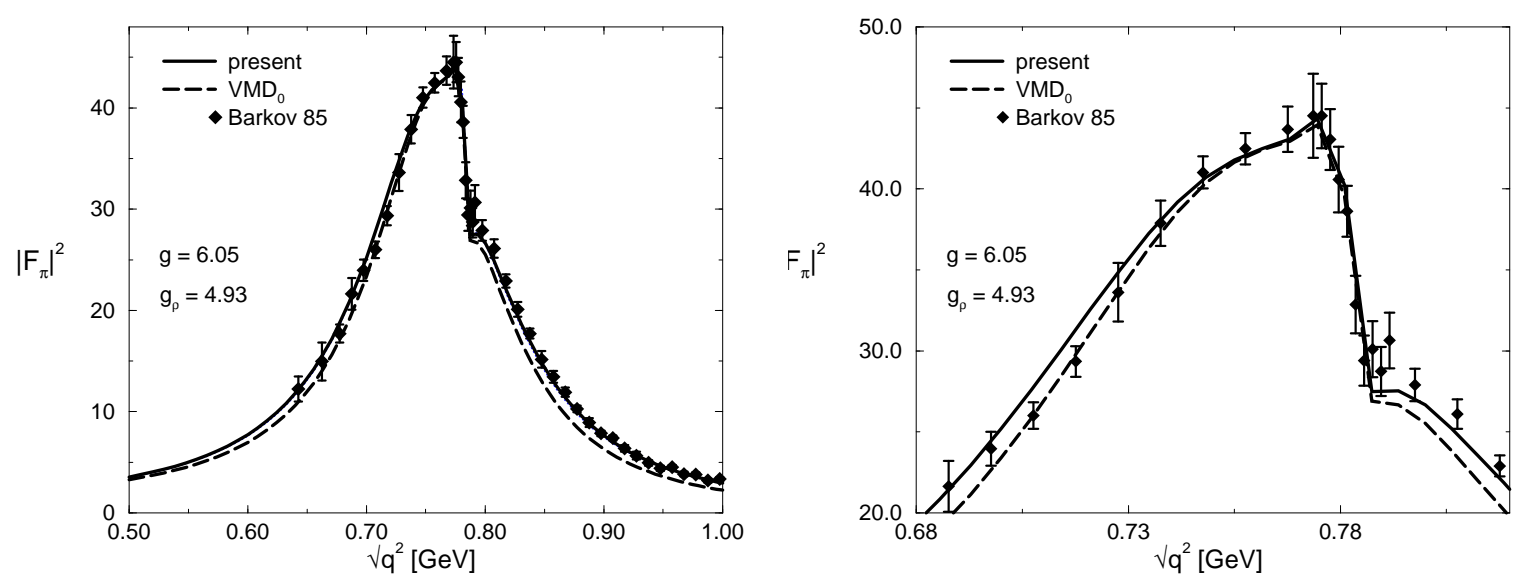

Figure 1.9.: Pion Form Factor (time-like) [Kli96].

Both figures show the same pion form factor $\left|F_{\pi}\left(q^{2}\right)\right|^{2}$ in the time-like region, the right figure is magnified. The experimental data points presented are from [Bar85].

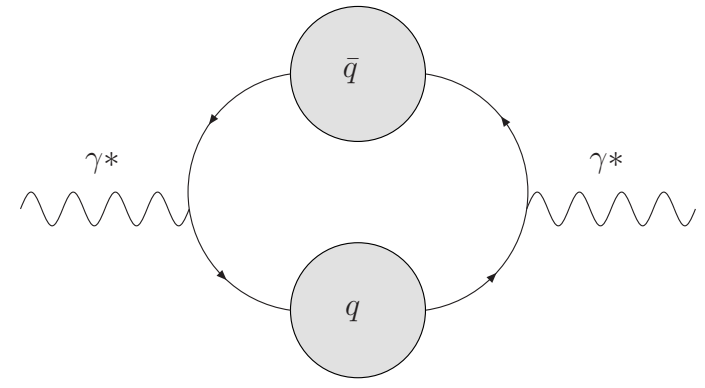

(a) hadonic characteristics of virtual photons

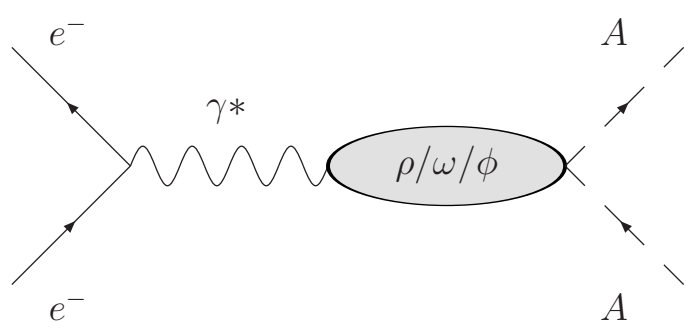

(b) VMD electron nucleon scatering

Figure 1.10.: Coupling of Virtual Photons to Hadrons in the VMD Model.

Figure (a) shows a virtual $\gamma^{*}$ forming (fluctuating into) an intermediate $q \bar{q}$ pair (time evolving to the right). The quantum numbers of a virtual photon $\gamma^{*}$ are $\left(J^{P C}\right)=\left(1^{--}\right)$, and thus a vector meson is formed, since its quantum numbers are identical (by definition) to quantum numbers of the photon. Figure (b) shows the coupling of such an intermediate vector meson in $e^{-}$-Hadron scattering (time axis upwards). By this mechanism the virtual photon acquires hadronic properties at small momentum transfers according to the Vector Meson Dominance (VMD) Model (see Section 1.4). 


\begin{tabular}{|c|c|c|c|c|c|}
\hline $\begin{array}{c}\text { Particle } \\
\text { Name }\end{array}$ & $\begin{array}{c}\text { Q. N. } \\
I^{G}\left(J^{P C}\right)\end{array}$ & $\begin{array}{c}\text { Mass } m \\
/ \mathrm{MeV}\end{array}$ & $\begin{array}{c}\Gamma \\
/ \mathrm{MeV}\end{array}$ & $\begin{array}{c}\text { Main Decay } \\
\text { Channel }\end{array}$ & $\begin{array}{c}\text { Branching Ratio } \\
\text { into } e^{+} e^{-}\end{array}$ \\
\hline \hline$\rho^{0}(770)$ & $1^{+}\left(1^{--}\right)$ & $775,5 \pm 0,4$ & 149,4 & $\approx 100 \%$ to $\pi \pi$ & $(4,70 \pm 0,08) \times 10^{-5}$ \\
\hline$a_{1}(1260)$ & $1^{-}\left(1^{++}\right)$ & $1230 \pm 40$ & $\approx 500$ & uncertain & uncertain \\
\hline$f_{0}(600)$ & $0^{+}\left(0^{++}\right)$ & $400-1200$ & $\approx 800$ & $\pi \pi$ & not seen so far \\
\hline$\omega(782)$ & $0^{-}\left(1^{--}\right)$ & $782,65 \pm 0,12$ & 8,49 & $\approx 89 \%$ to $\pi^{+} \pi^{-} \pi^{0}$ & $(7,18 \pm 0,12) \times 10^{-5}$ \\
\hline$\phi(1020)$ & $0^{-}\left(1^{--}\right)$ & $1019,46 \pm 0,02$ & 4,62 & $\approx 50 \%$ to $K^{+} K^{-}$ & $(2,97 \pm 0,04) \times 10^{-5}$ \\
\hline
\end{tabular}

Table 1.2.: Selected Light Mesons [Yao06a].

Q. N. abbreviates the Quantum Numbers, the meson spin $J$ is given by $|l-s|<J<|l+s|$ when $s=0$ (anti-parallel quark spin) or $s=1$ (parallel quark spin), Parity $P=(-1)^{l+1}, l$ is the orbital angular momentum; $C$-Parity $C=(-1)^{l+s}, G$-Parity $G=(-1)^{I+l+s}, I$ is the isospin and $I_{z}$ its z-component. The lifetime $\tau$ of these resonances/particles are related to their decay widths $\Gamma$ by $\tau=\frac{\hbar}{\Gamma}$.

\subsection{Dilepton Experiments}

The unique properties of vector mesons which are utilized in dilepton experiments (such as HADES, compare Chapter 2) are their short lifetime paired with their decay channel into dileptons. The short lifetime ensures their decay inside the hot and dense initial phase of a heavy ion collision, while their decay into a pair of leptons $\left(e^{+} e^{-}\right)$provides a conceptually ideal tool to study this phase undisturbed, since the lepton pairs can leave the hot and dense medium without further strong interaction (i.e. there is no strong final state interaction). In addition, the study of decays of vector mesons into channels with only a lepton pair (for example: $\rho^{0} \rightarrow e^{+}+e^{-}$) allows the direct reconstruction of the invariant mass of the vector meson at the point in space and time where its decay took place, i.e. in the hot and dense medium of the heavy ion collision. This provides an interesting tool to investigate in medium effects such as chiral restoration (compare Section 1.2.4).

Several experiments measured dileptons in different collisions at different energies up to now: for example DLS [Por97], CERES [Aga05], HELIOS [Maz94], NA60 [Arn06] and recently HADES [HAD94]. Spectra from two of them, HADES and NA60, are shown in Figure 1.11 and Figure 1.12. Prominent in all the experiments is that the so-called hadronic cocktail, which describes the spectrum as the naive sum of all known hadronic contributions which works perfectly well for proton-nucleus collisions, does not describe the data adequately. Instead a significant enhancement in the region of the $\rho / \omega$ peak relative to the hadronic cocktail has been observed.

Two different approaches are followed by theory to explain this: the dropping of the $\rho$ vector meson mass in the medium, as for example described in [Hun97, Cas95, Li96, Bro02], or the modification of the spectral function in medium, as described in [Bra98, Rap00, Urb98, Urb00, Rap97, Cha96]. The dropping $\rho$ mass scenario seems to be sufficient to explain the spectra presented by CERES, but a modern high statistics experiment (NA60) strongly supports the second scenario of the in-medium modification of the spectral function of the $\rho$ meson [Arn06]. Comparing the two models with the available experimental data, as shown in Figure 1.12, the NA60 collaboration completely rules out that the $\rho$ meson is not modified in any way as given by the dashed line. In addition, it is concluded, that an in medium mass drop of the $\rho$ meson does not describe the data as it is shown by the dashed-dotted line in Figure 1.12. The model that describes the NA60 data well is the strong broadening of the $\rho$ meson's spectral function 
shown by the thick solid line.

This result does not seem to rule out dropping mass scenarios of vector mesons in general, since other experiments with different conditions report a dropping mass. The in-medium modification of the $\omega$ mesons in the photo-production process $\gamma+A \rightarrow \omega+X \rightarrow \pi^{0} \gamma+X^{\prime}$ has been investigated by the CBELSA/TAPS collaboration [Trn05]. There, a drop of the in-medium mass of the $\omega$ meson to $M_{\omega}=722 \mathrm{MeV} / \mathrm{c}^{2}$ (equal to $8 \%$ drop) is reported at an estimated average nuclear density of $0,6 \cdot \rho_{0}$.

Summarized it seems that the matter of in medium modification still needs a lot of investigation before it will be finally concluded.
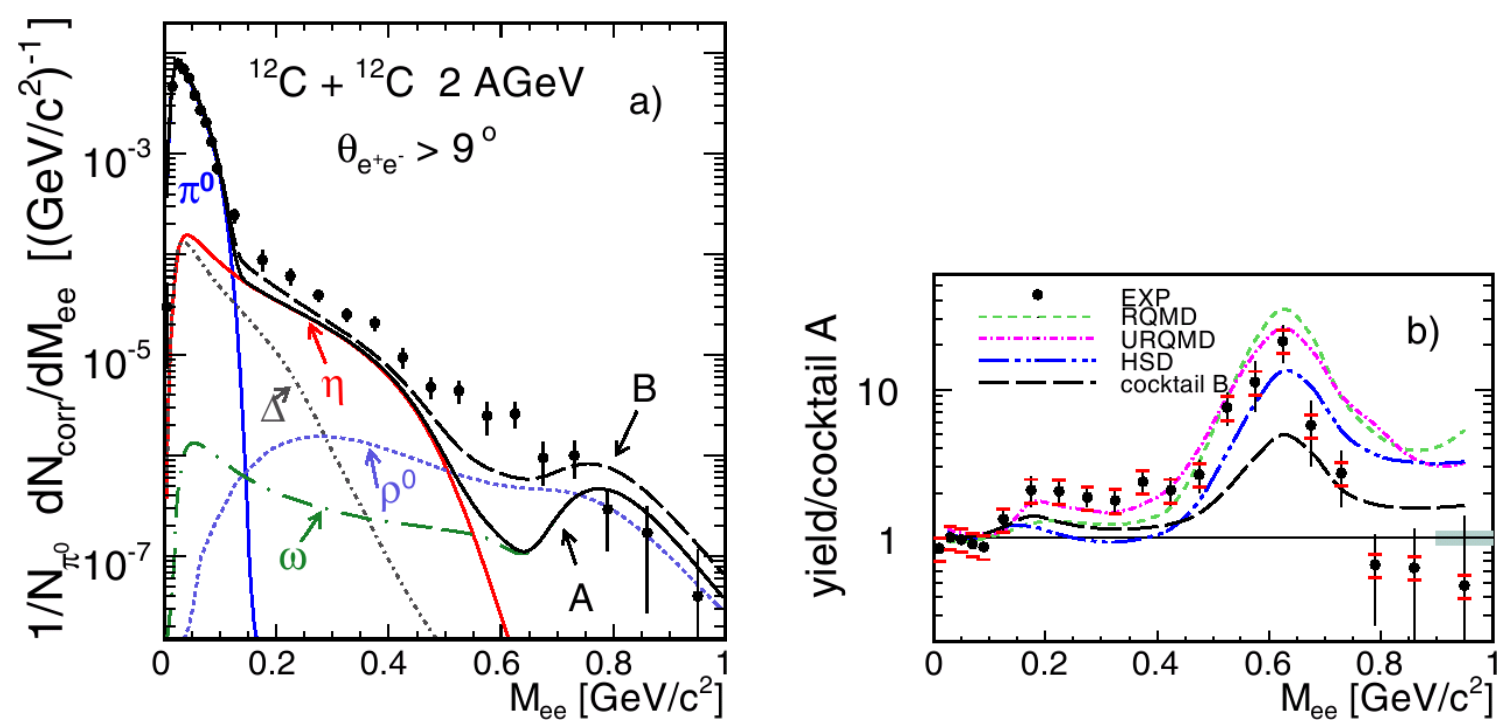

Figure 1.11.: Recent HADES Dilepton $\left(e^{+} e^{-}\right)$Spectra from C $+\mathbf{C}$ [Aga07].

The left figure shows the invariant $e^{+} e^{-}$-pair mass distribution data (dots with error bars) normalized to the $\pi$ yield, and shows for comparison two cocktail (A/B) with their different sources. Cocktail A is a thermal cocktail, while cocktail B also includes resonant decays (details in [Aga07]). The figure on the right compares the measured data with several theoretical models and the cocktails. It should be noted that the transport models qualitatively reproduce the measured curve, but fall short in describing the actual yield, most likely due to the use of vacuum spectral functions instead of in medium spectral functions. 

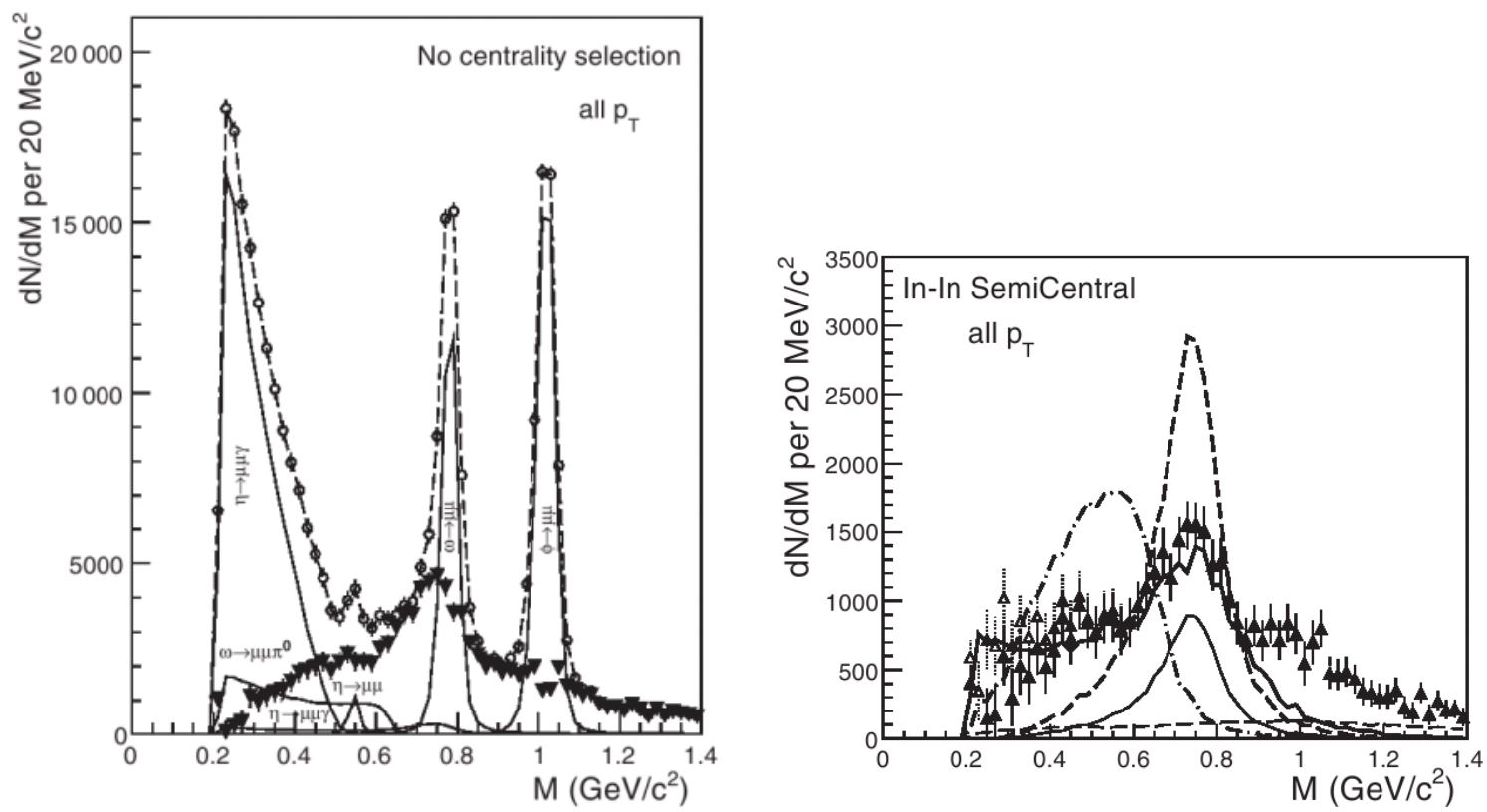

Figure 1.12.: NA60 Dilepton $\left(\mu^{+} \mu^{-}\right)$Spectra in In+ln Collisions [Arn06].

The left figure shows the total mass spectrum data (open circles) without a centrality selection and compares them to the known contributing sources (solid lines) to obtain the actual excess, which is given by the solid triangles. The figure on the right compares the excess mass spectra data in the semi-central bin to several model predictions. The thin solid line is the cocktail prediction (sum of known sources) and the dashed dotted line shows calculations from the inmedium moving $\rho$ meson mass model [Bro02, Bro91, Li95], and the in medium broadening model prediction as in [Cha96, Rap00, Rap97] is given by the thick solid line. The actual data points are given by the solid triangles with error bars. 


\subsection{Bibliography}

[Ach05] P. AcHARD et al.; Measurement of the running of the electromagnetic coupling at large momentum-transfer at LEP; Physics Letters B (2005); 623:26-36; doi:10.1016/j.physletb.2005.07.052

[Aga05] G. AgAKICHIEV et al.; $e^{+} e^{-}$-pair production in Pb-Au collisions at 158 GeV per nucleon; Eur. Phys. J. (2005); C 41:475-513

[Aga07] G. N. Agakichiev; C. Agodi; H. Alvarez-Pol et al. (HADES); Dielectron Production in ${ }^{12} \mathrm{C}+{ }^{12} \mathrm{C}$ Collisions at $2 \mathrm{AGeV}$ with the HADES Spectrometer; Physical Review Letters (2007); 98(5):052302; doi:10.1103/PhysRevLett.98.052302; arXiv: nucl-ex/0608031

[Arn06] R. Arnaldi; R. Averbeck; K. Banicz et al.; First Measurement of the $\rho$ Spectral Function in High-Energy Nuclear Collisions; Physical Review Letters (Apr 2006); 96(162302); doi:10.1103/PhysRevLett.96.162302

[Bal00] Gunnar S. BALI; QCD forces and heavy quark bound states (May 2000); HUB-EP99/67, arXiv:hep-ph/0001312v2

[Bar85] L.M. BARKov et al.; Electromagnetic Pion Formfactor in the Timelike Region; Nuclear Physics B (1985); 256:365-384

[Bra98] E. L. Bratkovskaya; W. Cassing; R. Rapp et al.; Dilepton production and $m_{T}$-scaling at BEVALAC/SIS energies; Nuclear Physics A (1998); 634:168; doi:10.1016/S0375-9474(98)00135-3

[Bro91] G. E. Brown and Mannque Rho; Scaling effective Lagrangians in a dense medium; Phys. Rev. Lett. (May 1991); 66(21):2720-2723; doi:10.1103/PhysRevLett.66.2720

[Bro02] G.E. Brown and Mannque Rho; On the manifestation of chiral symmetry in nuclei and dense nuclear matter; Physics Reports (2002); 363:85 - 171; doi:10.1016/S03701573(01)00084-9

[Cas95] W. Cassing; W. Ehehalt and C. M. Ko; Dilepton Production at SPS Energies; Physics Letters B (1995); 363:35; arXiv:hep-ph/9508233

[Cha96] G. Chanfray; R. Rapp and J. Wambach; Medium Modifications of the Rho Meson at CERN Super Proton Synchrotron Energies (200 GeV/nucleon); Phys. Rev. Lett. (Jan 1996); 76(3):368-371; doi:10.1103/PhysRevLett.76.368

[Cle98] J. Cleymans and K. Redlich; Unified Description of Freeze-Out Parameters in Relativistic Heavy Ion Collisions; Phys. Rev. Lett. (Dec 1998); 81(24):5284-5286; doi:10.1103/PhysRevLett.81.5284

[Ger89] P. Gerber and H. Leutwyler; Hadrons below the chiral phase transition; Nuclear Physics B (July 1989); 321(2):387-429; doi:10.1016/0550-3213(89)90349-0

[GM68] Murray Gell-Mann; R. J. Oakes and B. Renner; Behavior of Current Divergences under $S U_{3} \times S U_{3}$; Phys. Rev. (Nov 1968); 175(5):2195-2199; doi:10.1103/PhysRev.175.2195 
[Gut06] H. H. GutBrod et al. (Editors); FAIR Baseline Technical Report (Gesellschaft für Schwerionenforschung, 2006); ISBN 3-9811298-0-6

[HAD94] HADES Collaboration; A proposal for a High Acceptance Di-Electron Spectrometer (1994); GSI Darmstadt

[Hal84] Francis Halzen and Alan Douglas Martin; QUARKS AND LEPTONS: An Introductory Course in Modern Particle Physics (Wiley \& Sons, Inc., 1984); ISBN 0-471-88741-2

[Hun97] C. M. Hung and E. V. ShuRYAK; Dilepton and/or photon production in heavy ion collisions and the QCD phase transition; Phys. Rev. C (Jul 1997); 56(1):453-467; doi:10.1103/PhysRevC.56.453

[Ich03] H. Ichie; V. Bornyakov; T. Streuer et al.; Flux Tubes of Two- and ThreeQuark System in Full QCD*; Nuclear Physics A721 (2003); pages 899c-902; doi:10.1016/S0375-9474(03)01238-7

[Kli90] S. Klimt; M. Lutz and W. Weise; Chiral phase transition in the SU(3) Nambu and Jona-Lasinio model; Physics Letters B (October 1990); 249(3,4):386; doi:10.1016/03702693(90)91003-T

[Kli96] F. KLingL; N. KAISER and W. WeISE; Effective Lagrangian approach to vector mesons, their structure and decays; Z. Phys. A (1996); 356:193

[Koc95] VolKer Koch; Introduction to Chiral Symmetry; LBNL-Report 38000 (1995); arXiv: nucl-th/9512029

[Koc02] Volker Koch; Aspects of Chiral Symmetry; LBNL-39463 UC-413 (March 2002); URL http://theory.gsi.de/ knoll/Lecture-notes/koch_notes.pdf

[Li95] G. Q. LI; C. M. Ko and G. E. Brown; Enhancement of Low-Mass Dileptons in Heavy Ion Collisions; Phys. Rev. Lett. (Nov 1995); 75(22):4007-4010; doi:10.1103/PhysRevLett.75.4007

[Li96] G. Q. LI; C. M. Ko and G. E. BRown; Effects of in-medium vector meson masses on low-mass dileptons from SPS heavy-ion collisions; Nuclear Physics A (1996); 606:568; arXiv:nucl-th/9608040

[Mas95] M. Masera et al. (HELIOS/3); Dimuon Production below Mass $3.1 \mathrm{GeV} / \mathrm{c} 2$ in $p$-W and $S$-W Interactions at $200 \mathrm{GeV} / \mathrm{c} / \mathrm{A}$; Nuclear Physics A590 (1995); pages 93-102

[Maz94] M. A. MaZzoni et al.; Dimuon and vector-meson production in $p$ - $W$ and $S$ - $W$ interactions at $200 \mathrm{GeV} / \mathrm{c} /$ nucleon; Nuclear Physics (1994); A566:95c-102

[Nis00] RichaRD Nisius; The photon structure from deep inelastic electron photon scattering; Physics Reports (2000); 332:165-317

[Noe18] Emmy Noether; Invariante Variationsprobleme; Nachr. d. König. Gesellsch. d. Wiss. zu Göttingen, Math-phys. Klasse (1918); pages 235-257; arXiv:physics/0503066v1 
[Por97] R. J. Porter; S. Beedoe; R. Bossingham et al.; Dielectron Cross Section Measurements in Nucleus-Nucleus Reactions at 1.0A GeV; Phys. Rev. Lett. (Aug 1997); 79(7):1229-1232; doi:10.1103/PhysRevLett.79.1229

[Pov06] Bagdan Povh; Klaus Rith; Christipoh Scholz et al.; Particles and Nuclei An Introduction to the Physical Concepts (Springer-Verlag, 2006); 5th edition; ISBN 978-3-54036683-6

[Rap97] R. Rapp; G. Chanfray and J. Wambach; Rho meson propagation and dilepton enhancement in hot hadronic matter; Nuclear Physics A (1997); 617:472-495; doi:10.1016/S0375-9474(97)00137-1

[Rap00] R. RAPP and J. Wambach; Chiral Symmetry Restoration and Dileptons in Relativistic Heavy-Ion Collisions; ADV.NUCL.PHYS. (2000); 25:1; arXiv:hep-ph/9909229

[Ros50] M. N. Rosenbluth; High Energy Elastic Scattering of Electrons on Protons; Physical Review (August 1950); 79(4):615

[Sak69] J. J. Sakurai; Currents and Mesons; The University of Chicago Press (1969)

[Tas01] EnRICo TAssi; Measurement of dijet production in neutral current deep inelastic scattering at high $Q^{2}$ and determination of $\alpha_{S}$ at HERA; Ph.D. thesis; Universität Hamburg (2001); URL http://www-library.desy.de/cgi-bin/showprep.pl? desy-thesis-01-059

[Trn05] D. TRNKA et al. (CBELSA/TAPS Collaboration); Observation of In-Medium Modifications of the $\omega$ Meson; Physical Review Letters (2005); 94:192303; doi:10.1103/PhysRevLett.94.192303

[Urb98] M. Urban; M. Buballa; R. Rapp et al.; Momentum Dependence of the Pion Cloud for Rho Mesons in Nuclear Matter; Nuclear Physics A (1998); 641:433; arXiv: nucl-th/9806030

[Urb00] M. URban; M. Buballa; R. Rapp et al.; Modifications of the Rho Meson from the Virtual Pion Cloud in Hot and Dense Matter; Nuclear Physics A (2000); 673:357; arXiv:nucl-th/9910004

[Yao06a] W.-M. YaO et al.; Review of Particle Physics; Journal of Physics G (2006); 33:3194; Summary Tables of Particle Properties; doi:10.1088/0954-3899/33/1/001; URL http://pdg.lbl.gov

[Yao06b] W.-M. Yao et al.; Review of Particle Physics; Journal of Physics G (2006); 33:110 118; Quantum Chromodynamics and its Coupling; doi:10.1088/0954-3899/33/1/001; URL http://pdg.lbl.gov

[Yao06c] W.-M. YaO et al.; Review of Particle Physics; Journal of Physics G (2006); 33:97; Physical Constants; doi:10.1088/0954-3899/33/1/001; URL http://pdg.lbl.gov

[Yao06d] W.-M. YaO et al.; Review of Particle Physics; Journal of Physics G (2006); 33:1-1232; doi:10.1088/0954-3899/33/1/001; URL http://pdg.lbl.gov 
[Yao06e] W.-M. Yao et al.; Review of Particle Physics; Journal of Physics G (2006); 33:181-194; Structure Functions; doi:10.1088/0954-3899/33/1/001; URL http://pdg.lbl.gov

[Yao06h] W.-M. YaO et al.; Review of Particle Physics; Journal of Physics G (2006); 33:165172; Quark Model; doi:10.1088/0954-3899/33/1/001; URL http://pdg.1bl.gov 


\section{The HADES Spectrometer}

\section{Contents}

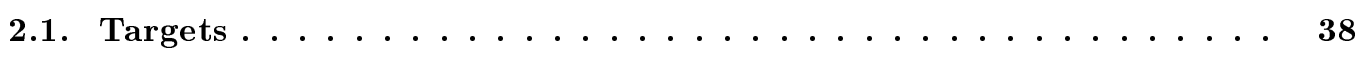

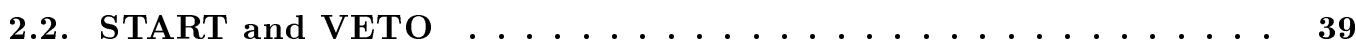

2.3. Ring Imaging C̆ERENKov Detector - RICH . . . . . . . . . . . 40

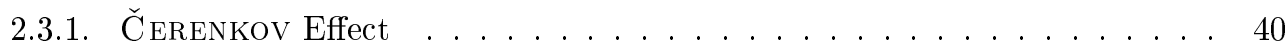

2.3.2. The HADES RICH . . . . . . . . . . . . . . . . . . 41

2.3.3. Online Ring Recognition . . . . . . . . . . . . . . . . . 43

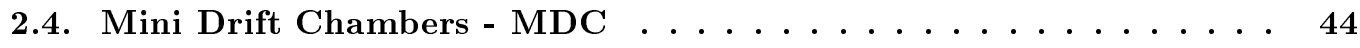

2.4.1. Physics and Characteristics of a Drift Cell . . . . . . . . . . . 47

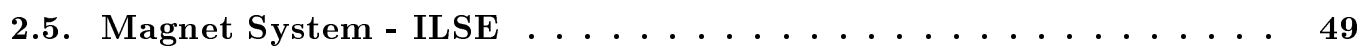

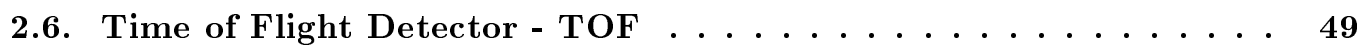

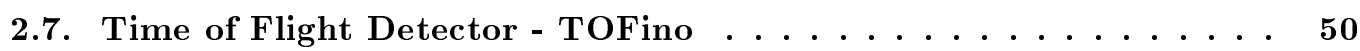

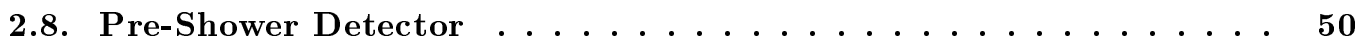

2.9. Resistive Plate Chambers Detector - RPC . . . . . . . . . 51

2.10. META System . . . . . . . . . . . . . . 52

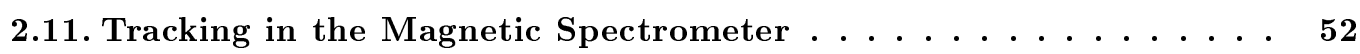

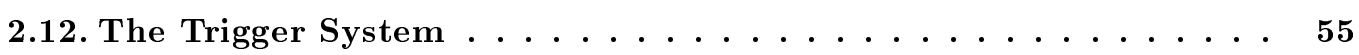

2.12.1. The First Level Trigger - LVL1 _ . . . . . . . . . . . . . 57

2.12.2. The Second Level Trigger - LVL2 . . . . . . . . . . . . . . . . . 57

2.12.3. Higher Trigger Levels (LVL3) and new Challenges . . . . . . . . . 57

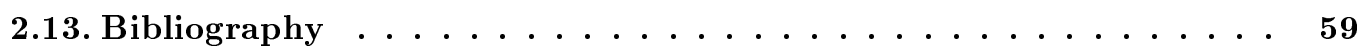

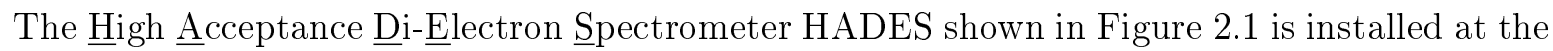
heavy ion synchrotron (SIS ${ }^{1}$ ) of the GSI $^{2}$ facilities in Darmstadt (Germany). Its main objective is the measurement of lepton pairs (dileptons) produced in the decay of light vector mesons at reaction rates of $10^{6} \mathrm{~Hz}$ with an accuracy of $\frac{\Delta p}{p} \simeq 1 \%$ particle momentum resolution [HAD94]. In order to fulfill the design goals the HADES spectrometer is composed of several different specialized particle detectors, which are described in detail in the following sections. The details of the detector setup of the spectrometer can be found in Figure 2.1 and Figure 2.2.

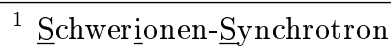

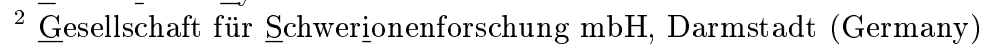




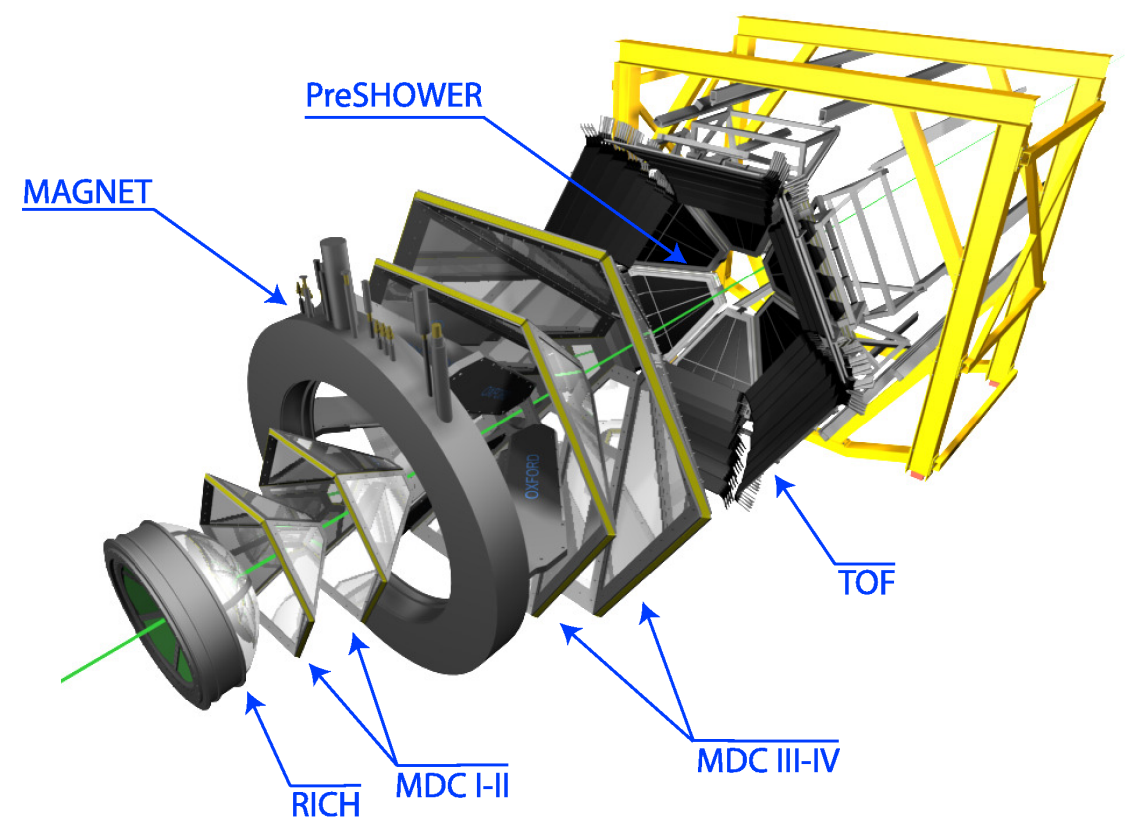

(a) exploded drawing of the HADES spectrometer

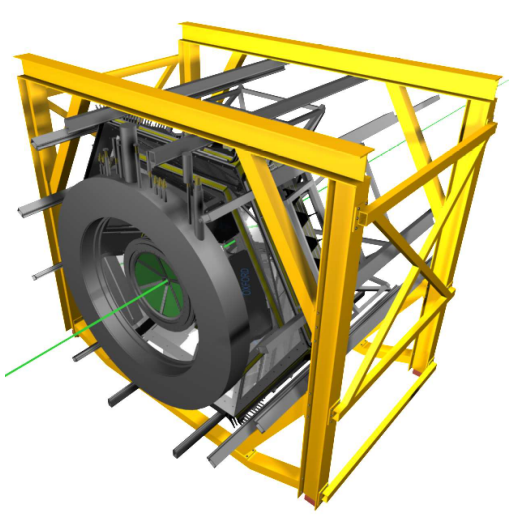

(b) view of the HADES spectrometer as mounted

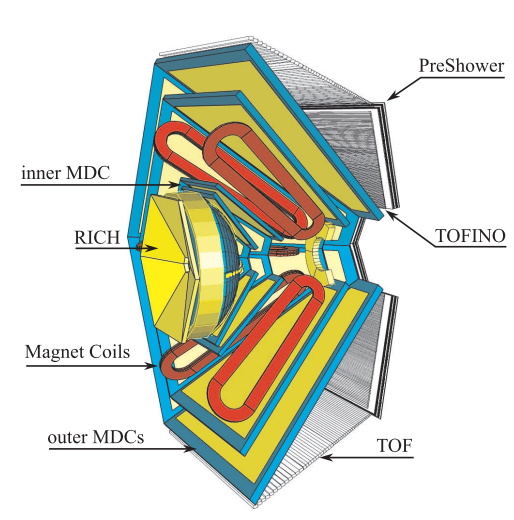

(c) cut through the assembled HADES spectrometer

Figure 2.1.: Artist's view of the HADES spectrometer [Sch06].

The view in figure a) is exploded to enhance the visibility of the individual detectors composing the spectrometer, while figures b) and c) show how tightly the detectors are mounted in reality inside the support frame (yellow). The green line entering from the bottom left represents the particle beam. The support frame's physical dimensions are about $5 \mathrm{~m}$ by $5 \mathrm{~m}$ by $4 \mathrm{~m}$ (width, height, depth). The sixfold symmetry of the whole setup is apparent. The definition of the coordinate system is: $z$-axis parallel to the beam direction, $y$-axis upward, $x$-axis to the left, polar angle $\vartheta$ is defined with respect to the $z$-axis and azimuthal angle $\phi$ is defined in the $x, y$-plane starting from the $x$-axis turning towards the $y$-axis. 


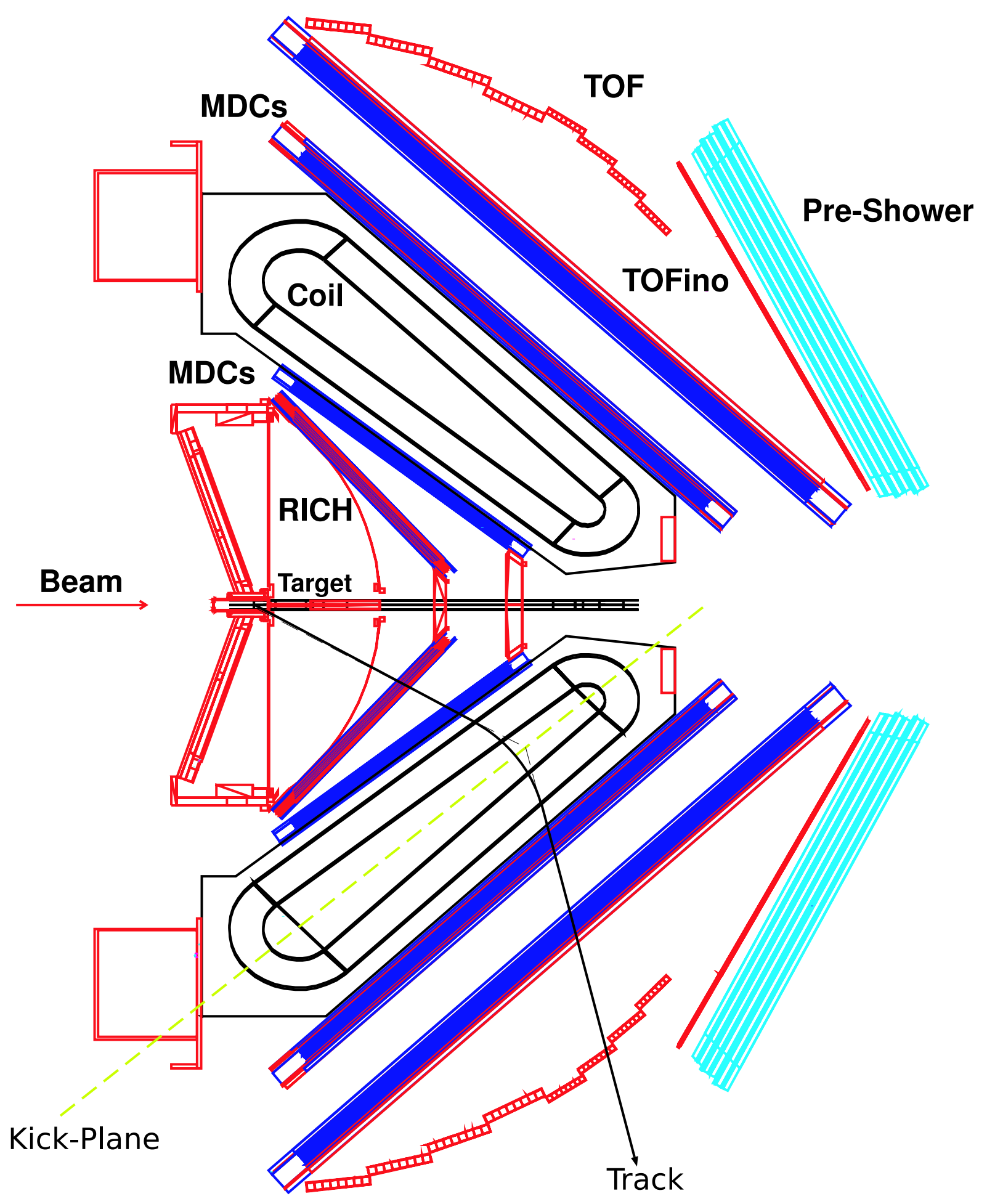

Figure 2.2.: Schematic of the HADES spectrometer shown in a lateral cut [HAD94].

Two of the six opposing sectors can be seen. The beam enters the spectrometer from the left side and hits the target. The magnet in the lower half of the picture has its kick-plane (compare Section 2.5) indicated by a dashed line. Also shown is the track of a charged particle. The track starts at the target, travels though the RICH and inner MDC chambers, is being bent in the magnet, and finally traverses the outer MDC chambers and hits the TOF. The bending in the magnet can be described by a momentum "kick" at the kick-plane (compare Section 2.5). 
Briefly summarized following the downstream direction of the beam, the HADES spectrometer features the HADES RICH for electron/positron particle identification (PID). Two sets of MDC drift chambers are mounted before and after a superconducting toroidal magnet respectively, to enable the tracking of charged particles and to measure their momentum. The setup is completed by the TOF and TOFino time of flight detector systems and the HADES Pre-Shower detector. These detectors serve particle identification and first level trigger event detection and form the

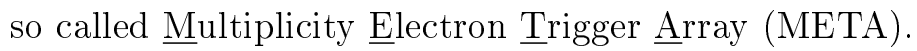

Light vector mesons decay into $e^{+} e^{-}$-pairs with a branching ratio of only about $10^{-5}$ [Yao06d]. In order to maximize the number of lepton pairs in the measured data samples, two approaches are realized by the HADES spectrometer: a large geometrical acceptance and high reaction rates. The former is realized by building a spectrometer which covers most of the $2 \pi$ azimuthal angle, and as a fixed target experiment gets most particles propagating downstream in the laboratory system. The latter is made possible only by a sophisticated multi-level trigger system described here as an integral part of the spectrometer. The overall geometrical acceptance of the spectrometer setup is approximately $80 \%$ of the full azimuthal angle and $18^{\circ}$ to $85^{\circ}$ in polar angle. This gives an overall result of about $40 \%$ acceptance for $e^{+} e^{-}$-pairs for the whole spectrometer.

The use of high primary beam rates of up to $10^{8}$ particles/s and a $1 \%$ interaction length target yields reaction rates of up to $10^{6} \mathrm{~Hz}$, which require a sophisticated real time trigger system to enrich the data sample with the rare decays of light vector mesons into dileptons. For example, a central $\mathrm{Au}+\mathrm{Au}$ collision at $1 \mathrm{AGeV}$ has a rate of dileptons from $\rho$-decays of only $10^{-6}$ pairs/event. In addition to these high rate requirements the trigger has to be very selective to handle the background from combinatorics and other channels as for example the conversion electrons from the photons of $\pi^{0}$ decays or the $\pi^{0}$ DALiTz decays.

The following sections provide more insight into the details of the operation of the individual detectors of the spectrometer and are ordered downstream starting with the target and ending with the PreShower. This is followed by the description of systems combining or interpreting data from individual detectors. The RICH and MDC sub detectors are of special interest for Chapter 4 "The Trigger Algorithm". For this reason, the RICH and MDC detectors are presented in more detail than the other detectors of the HADES spectrometer.

\subsection{Targets}

For different experiments, collisions of different particle species are studied. The projectile is given by the choice of an appropriate source used for the beam, and its well defined energy is given by the accelerator setup. The other partner in a fixed target experiment like HADES is mounted at the interaction point, typically either as thin foils or as a liquid.

Experiments with the goal to study the interaction of heavy ions can implement the target, as in the case of HADES, as (i) a single foil or (ii) segmented as a set of two or more foils. Case (i) was for example realized in the November 2001 beamtime $(\mathrm{C}+\mathrm{C} ; 1 \mathrm{AGeV}$ to $2 \mathrm{AGeV})$ with a single graphite foils with of $5 \mathrm{~mm}$ thickness, a density of $2,15 \mathrm{~g} / \mathrm{cm}^{3}$ and a interaction length of $5 \%$. Case (ii) was realized in the beamtime of November $2002(\mathrm{C}+\mathrm{C})$ with two graphite foils of $3 \mathrm{~mm}$ thickness, same density and a gap of $2 \mathrm{~cm}$ in between (placed $1 \mathrm{~cm}$ before and $1 \mathrm{~cm}$ after the nominal target position). A segmented target reduces the interactions (i.g. scattering) of reaction products with the target compared to a not segmented target of the same total thickness.

Experiments studying more elementary reactions with light projectiles (like pions and protons on a proton target) require a liquid hydrogen (LH2) target. The LH2 target of HADES is a 
cylindrical vessel (radius $=1,25 \mathrm{~cm}$, length $=5 \mathrm{~cm}$ ) made of Mylar foils. The liquid hydrogen is contained at atmospheric pressure at a temperature of $20 \mathrm{~K}$. Thermal isolation is provided by layers of aluminized Mylar foils. The used low $Z$ materials of the containment ensure a low interaction probability of the incoming beam with the container. For a proton beam the interaction probability with the container is $\approx 0,07 \%$, while the hydrogen target itself provides $\mathrm{a} \approx 1 \%$ interaction probability.

\subsection{START and VETO}

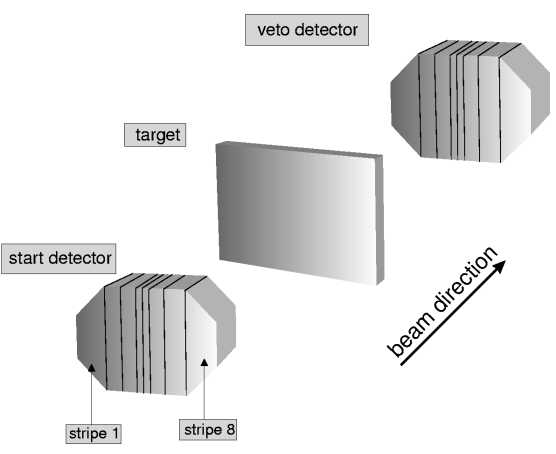

(a) Position of START and VETO

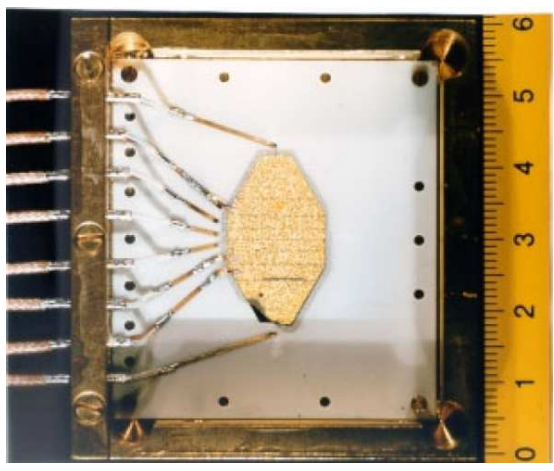

(b) START/VETO

Figure 2.3.: The START/VETO detectors.

In figure a) the position of the START and VETO detector relative to the target is outlined, while picture b) shows the real START/VETO (identical) detectors (ruler in $\mathrm{cm}$ ). The segmentation into stripes can be noted in both figures.

In order to decide if a reaction happened inside the target, the HADES spectrometer is equipped with a START and a VETO detector [Ber99]. Both detectors are CVD diamonds (Chemical Vapor Deposition) (thickness $=100 \mu \mathrm{m}$ ) of hexagonal shape divided into eight strips (as shown in Figure 2.3), with smaller inner strips to keep count rates constant among the different strips. The thickness is chosen to minimize multiple scattering. This type of detector is capable of high rates and can sustain the $10^{8}$ particles/s primary rate used in HADES. In addition, it provides a very good intrinsic time resolution of about $30 \mathrm{ps}$ and has a very good thermal conductivity.

START and VETO are each located $75 \mathrm{~cm}$ away from the target, START upstream and VETO downstream (compare Figure 2.3). They operate in anti-coincidence mode, i.e. a reaction is triggered, if a strip in the START detector detects a particle while no particle is detected in one of the three associated strips in the VETO detector (1:3 mode). In this case there is a high probability that the particle did not pass through the target without inducing a reaction. The efficiency for this START/VETO detector is $96,5 \%$ for heavy ion beams. In addition, the START detector provides beam focus monitoring and a spill monitor. For light particle beams the efficiency is much lower since the energy loss, and thus also the signal, depends on the $Z^{2}$ of the incident particle (Equation (2.3)). Therefore the START/VETO system was not used in the proton beam runs, i.e. January 2004 and May 2006. 


\subsection{Ring Imaging Čerenkov Detector - $\mathrm{RICH}$}

The first detector in downstream direction of the beam is the RICH (Ring Imaging CHERENKOV) detector which serves to discriminate leptons from hadrons. As the name implies, the ČERENKOV effect [Čer37b, Jac99] is used as the detection principle. This type of detector is often used in particle physics experiments [Seg94, Seg99, Yao06f] and is discussed in the following sections.

\subsection{1. Čerenkov Effect}

Whenever a charged particle travels through a medium (index of refraction $n$, vacuum speed of light $c$ ) with a velocity $\nu$ higher than the phase velocity of light $c_{n}$ in that medium, light is emitted along the path of the particle [Čer37b, Jac99, Yao06g].

$$
\nu>c_{n}=\frac{c}{n}
$$

This shows that the emission of ČERENKOV light starts at a threshold velocity $v_{t h r}$ and a threshold relativistic $\beta_{t h r}$ factor given by:

$$
\beta_{t h r}=\frac{1}{n}=\frac{\nu_{t h r}}{c}
$$

The ČERenKov light is emitted under an angle $\cos \Theta_{C}=\frac{1}{n \beta}$ and is totally linearly polarized in the plane containing the path of the particle and the direction of observation [Jac99]. Selecting the index of refraction by choosing an appropriate radiator material it is possible to precisely set the threshold velocity $\nu_{t h r}$ or the equivalent relativistic $\gamma_{t h r}$ factor (particle Energy $E$, rest mass $m)$ :

$$
\gamma_{t h r}=\frac{E}{m}=\frac{1}{\sqrt{1-\beta_{t h r}^{2}}}=\frac{1}{\sqrt{1-\left(\frac{1}{n}\right)^{2}}}
$$

The differential photon yield per energy is given by [Yps94]:

$$
\frac{\mathrm{d} N_{p h}}{\mathrm{~d} E}=\left(\frac{\alpha}{\hbar c}\right) \cdot Z^{2} L \cdot\left[1-\left(\frac{1}{\beta n}\right)^{2}\right]
$$

Here $\alpha$ is the fine structure constant, $\hbar$ is the Planck constant, $L$ is the path length of the particle traveling through the medium and $Z$ is the charge of the particle measured in units of the electron charge $e$. In case of constant $\beta n$ the photon yield $N$ can be written as

$$
\begin{gathered}
N=N_{0} Z^{2} L \sin ^{2} \Theta_{C} \\
N_{0}=\left(\frac{\alpha}{\hbar c}\right) \epsilon \Delta E \\
\epsilon \Delta E=\int_{E_{l}}^{E_{t}}(Q T R) \mathrm{d} E
\end{gathered}
$$

with $\epsilon$ being the energy average of the detector efficiency between the lower energy limit $E_{l}$ and the top energy limit $E_{t}$ given by the detector properties. 
The efficiency $\epsilon$ depends on the different photon efficiencies of the detector for transmission in the medium $T$, reflection on a mirror $R$ and quantum efficiency of detection $Q$ and $N_{0}$ is called the detector response parameter. It should be noted that in reality the index of refraction is frequency dependent. This causes a rise in the UV-region of the spectral distribution of the ČERENKOV light.

\subsubsection{The HADES RICH}

The layout of the RICH detector of HADES [Zei99a] is shown in Figure 2.4. Its design goal is to provide the data to perform the online identification of $e^{+} e^{-}$pairs in an environment of about 200 charged particles from heavy ion collisions at rates of $10^{5}$ interactions/s. As seen in Figure 2.1 and Figure 2.2, the detector is placed around the target and covers almost the full hemisphere in the forward direction.

This detector uses $\mathrm{C}_{4} \mathrm{~F}_{10}$ gas $(n=1,00151)$ as radiator material for its fitting $\gamma_{t h r}$, its good photon transmittivity and low energy loss. The use of Equation (2.1) leads to a threshold of $\gamma_{t h r}=18,22$ and also to the minimum particle energy required to produce ČERENKOV light for different particle species, for example:

$$
E_{e^{+}}=E_{e^{-}} \approx 9,3 \mathrm{MeV} ; \quad E_{\pi} \approx 2,6 \mathrm{GeV} ; \quad E_{p} \approx 17,8 \mathrm{GeV} ; \quad E_{\mu} \approx 1,92 \mathrm{GeV}
$$

This demonstrates that at the maximum SIS energy of $2 \mathrm{AGeV}$ for heavy ions basically only electrons are above this $\gamma_{t h r}$. It should be noted however, that for example in the case of a $2 \mathrm{AGeV}{ }^{12} \mathrm{C}$-beam the total available energy in the collision is actually high enough to produce also particles other than electrons above the $\gamma_{t h r}$. However, this is not a process with a high probability. Thus, only few pions with energies above the threshold are produced, and their ring diameter is smaller than for electrons (see below). The ring detection algorithms in HADES only find rings of the asymptotic size of the electron rings .

Since $\beta$ approaches 1 for electrons and positrons, the light is emitted under an asymptotic angle of $\Theta_{C}=3,15^{\circ}$. The mirror reflects this light as a ring of approximately constant diameter onto the photo detector plane. The inclination of $20^{\circ}$ of the detection plane is a compromise which helps to keep the reflected light approximately focused onto the plane independent of the angle of the particle track. The projected rings have a diameter of about $5 \mathrm{~cm}$ [Leh00].

The photo detector plane is segmented into 4712 pads per sector, and the pad size is varied along the $y$-axis from $7,1 \mathrm{~mm} \times 6,6 \mathrm{~mm}$ to $4,6 \mathrm{~mm} \times 6,6 \mathrm{~mm}$ to keep the size of a ring approximately constant (Figure 2.5) and correct for eccentricity effects modifying the ring shape, which arise from using a flat detection plane instead of a complicated proper shaped plane [Sch95]. This fixes the ring size to a radius of 4 pads [Leh99, Leh00] (compare Figure 2.5).

The detection of photons is realized by means of a MWPC ${ }^{3}$ with a CsI photo-cathode and $\mathrm{CH}_{4}$ as detector gas, located at backward angles in the field free region of the spectrometer. The optical mirror [Fri03] is composed of carbon, coated with an $\mathrm{Al}$ reflective layer and a $\mathrm{MgF}_{2}$ protective layer. Its physical thickness is $2,3 \mathrm{~mm}$ which results in a radiation thickness of $x / X_{0}<$ $1 \%$. The reflectivity is approximately $80 \%$. The optical window separates the radiator gas volume and the photo-detector gas volume. It is composed of $\mathrm{CaF}_{2}$ and has a transmittivity of approximately $70 \%$.

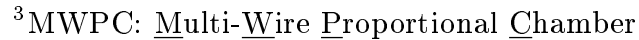




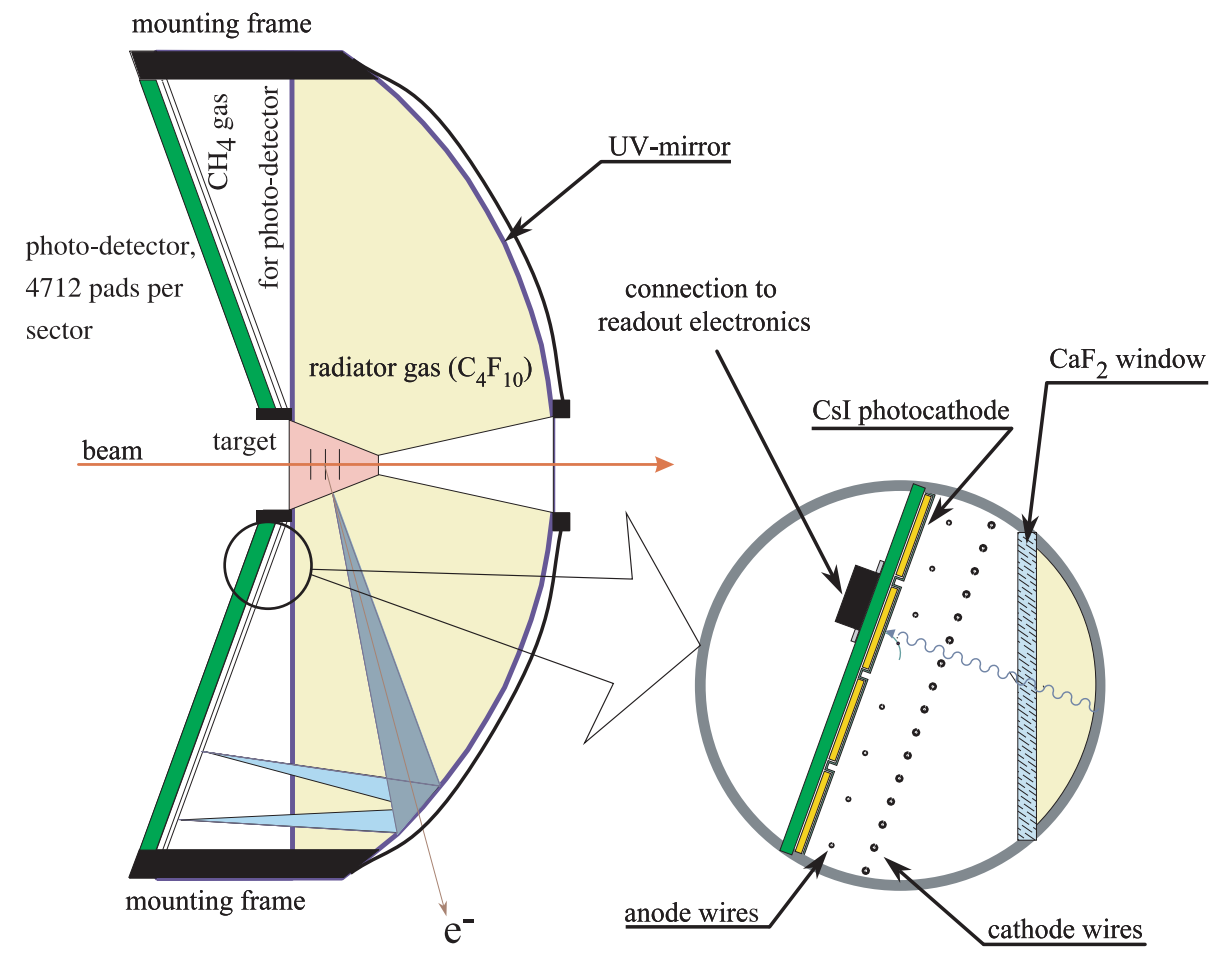

Figure 2.4.: Schematic lateral cut of the RICH detector of HADES [Böh99].

The beam is entering from the left and hits the target. Electrons with momenta of $p_{e} \geq 10 \mathrm{MeV} / \mathrm{c}$ traveling through the radiator gas $\left(\mathrm{C}_{4} \mathrm{~F}_{10}\right)$ produce ČERENKOV light in a cone along the particle path. This light is reflected by a mirror and focused in a ring shape onto the photon detector,

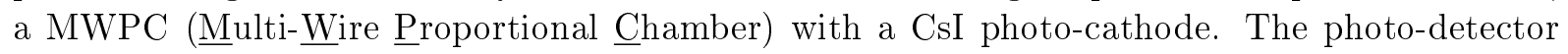
gas $\left(\mathrm{CH}_{4}\right)$ volume and the $\mathrm{C}_{4} \mathrm{~F}_{10}$ radiator gas volume are separated by a $\mathrm{CaF}_{2}$ window. The outer diameter of the RICH is about $1,58 \mathrm{~m}$ and the inner gap for target and beam pipe has a diameter of $15 \mathrm{~cm}$. The center of the target is located nearly in the focal point of the mirror. For further details please refer to Section 2.3.

Since ČERENKOV light is more intense at higher frequencies, all the components of the HADES $\mathrm{RICH}$ are chosen and optimized to operate at highest efficiency in the VUV ${ }^{4}$. The wavelength dependence of all relevant components is shown in Figure 2.5, which provides with Equation (2.2) the figure of merit for the RICH to $N_{0}=109$ [Zei99a]. The number of ČERENKOV photons generated when a particle travels through the RICH can be estimated by the formulas in Section 2.3.1. This also provides the average number of photons available for detection. It mainly depends on the path length of the particle in the radiator material and by this on the polar angle. Finally, it ranges from $\approx 7$ photons at a path length of $L=0,38 \mathrm{~m}$ at $\theta=20^{\circ}$ to $\approx 14$ photons at a path length of $L=0,68 \mathrm{~m}$ at $\theta=80^{\circ}$ [Sal02]. The quantum efficiency of the photo detector as limiting component is also clearly visible in Figure 2.5.

\footnotetext{
${ }^{4}$ VUV or FUV: vacuum or far UV ( $\underline{\text { Ultra }} \underline{\text { Violet}}$ ) light with a wavelength of approximately $200 \mathrm{~nm}$ to $10 \mathrm{~nm}$
} 


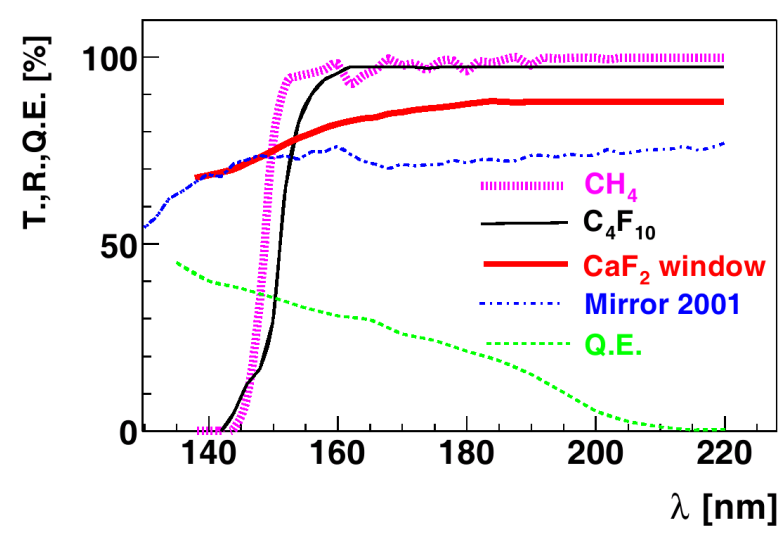

(a) Optical Efficiency RICH components

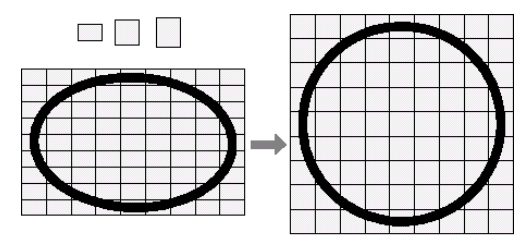

(b) RICH pad size variation

Figure 2.5.: Optical Parameters of the HADES RICH components [Fab03a, Fab03b, Leh00].

Figure a) shows the transmittivity (T.) of $\mathrm{CH}_{4}$ detector gas volume, the transmittivity of the $\mathrm{C}_{4} \mathrm{~F}_{10}$ radiator gas volume, the transmittivity of the $\mathrm{CaF}_{2}$ optical window and the reflectivity (R.) of the mirror and the quantum efficiency (Q.E.) of the CsI MWPC, all shown in percent depending on the wavelength $\lambda$. Figure b) shows the variation of the RICH photo-detector pad size to correct the ring shape.

As part of the online trigger system, the HADES RICH features a special hardware module, the RICH IPU (Image Processing Unit), which performs a search for the images of rings on the readout plane and provides the data of the found ring centers (i.e. $(\vartheta, \phi)$ of an electron or positron) in real-time [Leh00].

\subsubsection{Online Ring Recognition}

The RICH RRU (iing Recognition $\underline{\text { Unit}) ~[L e h 00, ~ L e h 99] ~ i s ~ a ~ d e d i c a t e d ~ h a r d w a r e ~ m o d u l e ~ r e-~}$ sponsible for extracting the $(\vartheta, \phi)$-angle information of a electron or positron track based on the data of the RICH detector. It performs this analysis in real time (online) as part of the LVL2 trigger system (compare Section 2.12). The angle information is given by the reconstructed centers of the ring shaped images of the ČERENKOV light produced in the RICH and read out on its photo-detector plane. The search for the rings and their centers is performed online by the RRU, a highly parallel processing FPGA ${ }^{5}$ based system. The method used is outlined and explained in the caption of Figure 2.6. One RRU per sector is combined with its PRC (Pattern

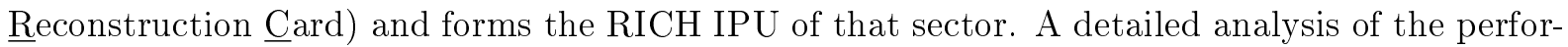
mance of the RICH IPU system as part of the LVL2 trigger of HADES can be found in [Leh99] and [Leh03].

\footnotetext{
${ }^{5}$ FPGA: Field Programmable Gate $\underline{\text { Array }}$
} 


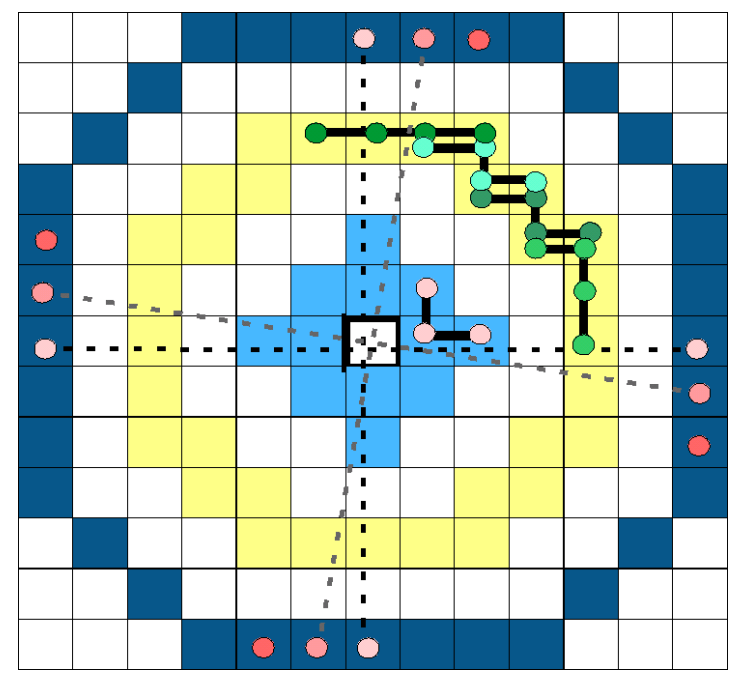

Figure 2.6.: Ring Recognition Search Mask and Pad Groups [Leh00, Leh03].

The online ring search in the RICH IPU is performed by processing all the possible 13 by 13 pads sub-arrays of the photo-detector plane. Each sub-array is divided into a ring region (yellow, fixed ring radius 4 pads) and an inner (light blue) and outer veto region (dark blue, outer radius 6 pads). Pads shown in white are not taken into account in this sub-array, but belong to the veto or ring region in another sub-array. To improve the efficiency of the implementation the pads inside the different regions are grouped into logical or-groups, telling if there was at least one pad fired in the group. The sum of the or-groups per region is calculated and compared to given threshold settings. A ring candidate is found if the count of the ring region exceeds the threshold while the veto region does not. Since this pattern search produces multiple candidates in sub-arrays with neighboring centers it is followed by a local maximum search comparing the counts in the ring region, and is thus providing the found rings. A found ring is defined by its ring center position on the pad plane which can be directly recalculated into the $(\vartheta, \phi)$ - angle information of an electron or positron track. Fakes due to electronic and detector noise, caused by undesired secondary effects (e.g. radiation in the MWPC) are not taken into account.

\subsection{Mini Drift Chambers - MDC}

The tracking of charged particles in HADES is accomplished by the so called MDC (Mini $\underline{\text { Drift }}$ Chambers) detector [Gar98, Mün04]. The name arises from the comparably small size of the individual drift cells. Figure 2.1 and Figure 2.2 show the positions of the four MDC chambers (MDC modules) per sector in the setup. The MDC system covers nearly the full azimuthal angle and the polar angle from $\vartheta=18^{\circ}$ to $\vartheta=85^{\circ}$, and it was designed as a "low-mass" system.

Two MDC modules per sector are located before the magnetic field and two behind the magnetic field, for a total of 24 trapezoidal chambers. The chambers are organized into four sets of six identical chambers. The sets are numbered I to IV, I being the first set in forward direction, i.e. module I and II are before the magnet, and are referred to as inner MDC chambers, while module III and IV are behind the magnet, and are called outer MDC chambers. The chambers are also numbered from 1 to 6 in the order of increasing azimuthal angle. The MDC modules with the number 1 being at $90^{\circ}$ (up) in sector $1^{6}$.

\footnotetext{
${ }^{6}$ Software framework convention is to number sectors from 0 to 5 . Compare Chapter 4.
} 


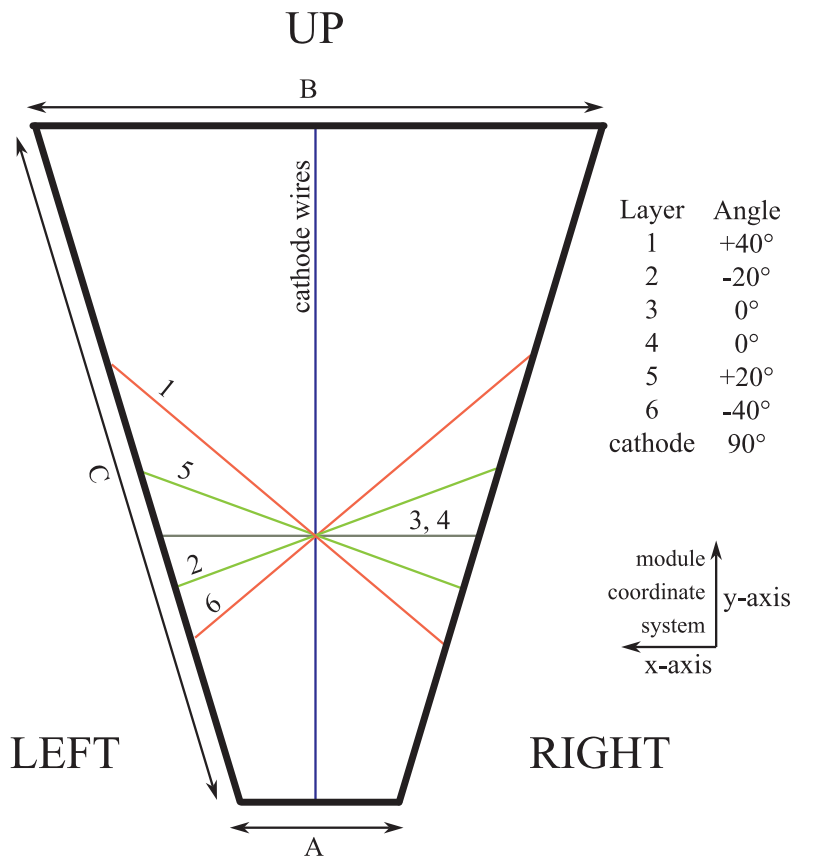

DOWN

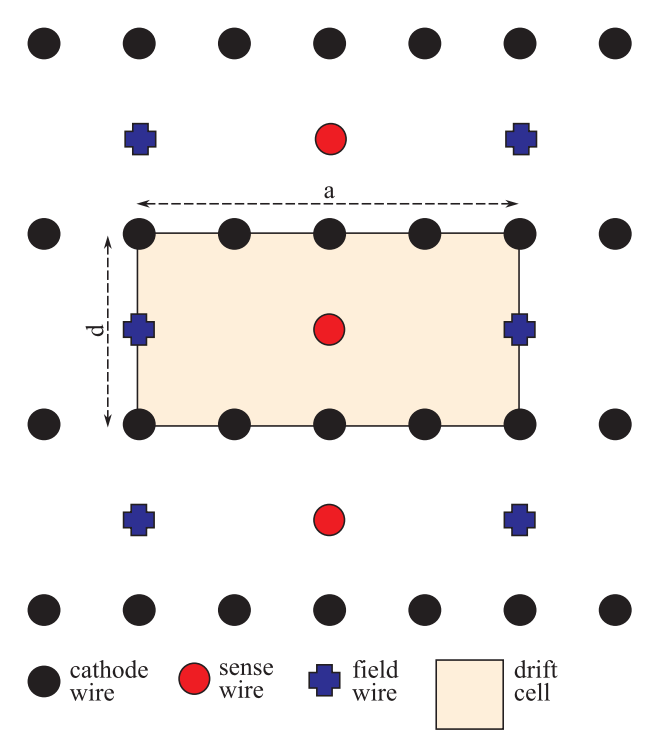

(a) MDC module schematic

(b) MDC wire arrangement

Figure 2.7.: Wire layout in the MDC modules.

The general arrangement of the wires in a MDC module is shown in a). The different inclinations of the layers of sense and field wires are shown as an example for a single wire of each layer. The definition of the module coordinate system can also be noted. All cathode wires are at an angle of $90^{\circ}$. The figure in b) sketches the relative position of the different wire types with respect to each other and shows the definition of the MDC drift cell, which extends out of and into the drawing plane to form a long box centered around the sense wire and delimited by the field wires and the cathode wires. Note that the angle between the cathode wires and the drift cell given in a) is not shown in b). The physical dimensions $(A, B, C, a, d)$ of the different MDC modules can be found in Table 2.1 and Table 2.2.

Each MDC module is composed of a stable stesalit frame holding the wires of the layers under a well defined tension. To optimize the acceptance the frames of the chambers are located in the shadows of the coils of the magnets. Sense wires and field wires are arranged in six layers, separated from each other by an interposed layer of cathode wires.

A drift cell is a box defined by the sense wire in the center delimited by two field wires and the cathode plane as shown in Figure 2.7. This figure also shows how the drift cells are arranged in planes with different inclination angles. A MDC module has about 190 drift cells or sense wires per layer (actual number varies per module and layer), numbered counting from the one at the most down position (Figure 2.7, smallest y). The entry and exit window of the chambers are $12 \mu \mathrm{m}$ thick aluminum coated Mylar foils [Gar98]. A helium-isobutane mixture in a ratio of $40: 60$, continuously monitored and purified, is used as counting gas to keep the total thickness 
of the MDC detector below $5 \cdot 10^{-4}$ in units of the radiation length.

The different MDC modules have different characteristics depending on their position in the spectrometer. A summary of this can be found in Table 2.1 and Table 2.2. The small drift cell sizes have been chosen to enhance the high event rate response capability by minimizing the ionization drift times by minimizing the path length (compare also Section 2.4.1). The data provided by this detector are the numbers of the drift cells which did see a signal and the associated drift times for these hits.

\begin{tabular}{|c|c|c|c|c|c|c|c|c|}
\hline Module & $A / \mathrm{mm}$ & $B / \mathrm{mm}$ & $C / \mathrm{mm}$ & $\gamma /{ }^{\circ}$ & $a / \mathrm{mm}$ & $d / \mathrm{mm}$ & Area $/ \mathrm{m}^{2}$ & Volume $/ 1$ \\
\hline \hline I & 139,21 & 767,38 & 839,19 & 21,98 & 5 & 5 & 0,34 & 11,99 \\
\hline II & 205,00 & 905,00 & 1049,27 & 19,49 & 6 & 5 & 0,49 & 20,58 \\
\hline III & 310,43 & 1804,80 & 2139,05 & 20,44 & 12 & 8 & 1,88 & 150,4 \\
\hline IV & 345,46 & 2224,05 & 2689,04 & 20,44 & 14 & 10 & 2,83 & 197,8 \\
\hline
\end{tabular}

Table 2.1.: Characteristics of the different MDC Modules [Str98].

The meaning of the parameters $(A, B, C, a, d)$ is given in Figure 2.7. The angle $\gamma$ is defined between the normal vector of the surface of the chamber and the $z$-axis.

\begin{tabular}{|c|c|c|c|}
\hline Module & $\varnothing$ cathode wire $(\mathrm{Al})$ & $\varnothing$ field wire $(\mathrm{Al})$ & $\varnothing$ signal wire $(\mathrm{Au}$ on $\mathrm{W})$ \\
\hline \hline I & $80 \mu \mathrm{m}$ & $80 \mu \mathrm{m}$ & $20 \mu \mathrm{m}$ \\
\hline II & $80 \mu \mathrm{m}$ & $80 \mu \mathrm{m}$ & $20 \mu \mathrm{m}$ \\
\hline III & $80 \mu \mathrm{m}$ & $100 \mu \mathrm{m}$ & $20 \mu \mathrm{m}$ \\
\hline IV & $100 \mu \mathrm{m}$ & $100 \mu \mathrm{m}$ & $30 \mu \mathrm{m}$ \\
\hline
\end{tabular}

Table 2.2.: Properties of the MDC-wires [Mün04].

Cathode and field wires are made of aluminum. Gold plated tungsten is used for the signal wires. 


\subsubsection{Physics and Characteristics of a Drift Cell}

The signal induced on the individual sense wires of the drift cells is the collected charge over time. The charge in form of electron-ion pairs is generated in clusters along the trail of a charged particle passing through the gas volume, and it is proportional to the energy loss of the incident particle.

In the relevant energy regime for this application, and for particles heavier than electrons, this ionization is the result of energy loss mainly via the BeTHE-BLOCH mechanism. This is described by the following formulas [Yao06g]:

$$
\begin{aligned}
-\frac{\mathrm{d} E}{\mathrm{~d} x} & =K z^{2} \cdot \frac{Z}{A} \frac{1}{\beta^{2}} \cdot\left[\frac{1}{2} \ln \left(\frac{2 m_{e} c^{2} \beta^{2} \gamma^{2} T_{\max }}{I^{2}}\right)-\beta^{2}-\frac{1}{2} \delta(\beta \gamma)\right] \\
T_{\max } & =\frac{2 m_{e} c^{2} \beta^{2} \gamma^{2}}{1+\frac{2 \gamma m_{e}}{M}+\left(\frac{m_{e}}{M}\right)^{2}}=\text { Maximum kinetic energy transfer per collision } \\
\alpha & =\frac{e^{2}}{4 \pi \epsilon_{0} \hbar c}=\text { Fine structure constant } \\
K & =4 \pi N_{A} r_{e}^{2} m_{e} c^{2} \\
M & =\text { Mass of the incident particle } \\
E & =\text { Energy of the incident particle } \\
T & =\text { Kinetik energy } \\
m_{e} & =\text { Electron mass } \\
c & =\text { Speed of light } \\
N_{A} & =\text { AvoGADRO's Number } \\
z e & =\text { Charge of the incident particle } \\
Z & =\text { Atomic number of Absorber } \\
A & =\text { Atomic mass of Absorber } \\
I & =\text { Mean exitation energy } \\
\delta(\beta \gamma) & =\text { Density effect correction } \\
\beta & =\frac{v}{c}=\text { velocity in units of } c
\end{aligned}
$$

For $\left(2 \gamma m_{e} / M\right) \ll 1$ the low energy approximation $T_{\max }=2 m_{e} c^{2} \beta^{2} \gamma^{2}$ can be used. Radiative effects are not relevant at the given energy regime.

In contrast, electrons above a critical energy $E_{c}$ lose energy mainly via Bremsstrahlung at a rate proportional to their energy, while energy loss due to ionization depends on the logarithm of the electron energy [Yao06g]. The approximation $E_{c}=(800 \mathrm{MeV}) /(Z+1,2)$ gives the energy where both effects are of equal strength, which is around a couple of $10 \mathrm{MeV}$. Photons generated by Bremsstrahlung also interact via further secondary processes and also produce ionization.

The charge deposited in the gas volume from direct ionization processes (primary ionization) is much lower than the charge due to all the secondary ionization effects. The sum of primary and secondary ionization, the total ionization, is what is collected by the sense wire after drifting in the drift cell. 


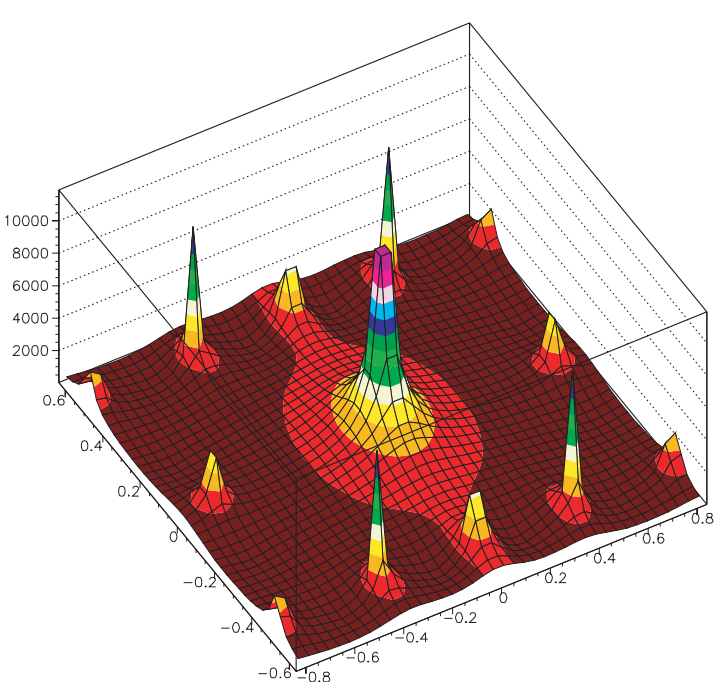

(a) Electric Field Configuration

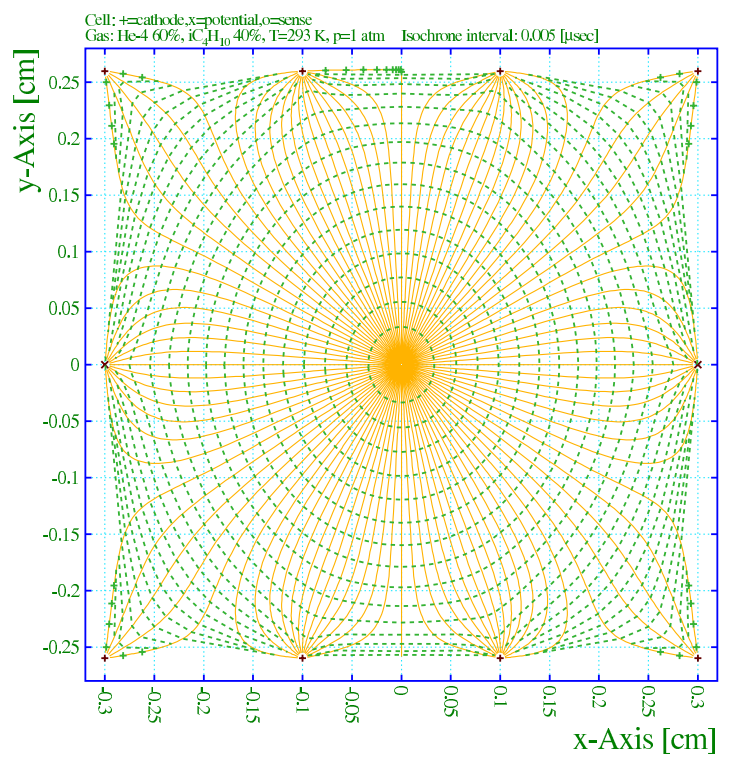

(b) Drift Time Isochrones

Figure 2.8.: MDC Drift Cell Characteristics [Mar05].

An Example contour plot of the electric field $\vec{E}$ in a drift cell for MDC IV of HADES is shown in a). The $x$-axis and $y$-axis are in $\mathrm{cm}$, while the $z$-axis is showing $\mathrm{V} / \mathrm{cm}$. The position of the peak in the center corresponds to the position of the sense wire. Figure b) shows lines of equal drift time for MDC II of HADES in case of a cathode/field wire potential of $-1800 \mathrm{~V}$. The time difference between the sketched isochronous lines is $\Delta t=5 \mathrm{~ns}$ and the position of the sense wire is also in the center.

The ionization electron drift is described by [Yao06f]:

$$
\vec{u}=\mu|\vec{E}| \frac{1}{1+\omega^{2} \tau^{2}}\left(\vec{e}+\omega \tau(\vec{e} \times \vec{b})+\omega^{2} \tau^{2}(\vec{e} \cdot \vec{b}) \vec{b}\right)
$$

Here $\vec{u}$ is the velocity vector of the electrons, $\vec{E}$ and $\vec{B}$ are electric and magnetic field and $\vec{e}$ and $\vec{b}$ are their unit vectors. The cyclotron frequency is given by $\omega=e B / m_{e} c$ and $\tau=\mu m_{e} / e$ is the mean time between collisions, with $\mu$ being the electron mobility in the gas. In HADES the electrons drift with a velocity of approximately $4 \mathrm{~cm} / \mu$ s along the electric field lines, as shown in Figure 2.8.

Close to the center of the drift cell, i.e. close to the thin sense wire, the gradient of the electric potential is strong enough to create an avalanche of electrons (compare Figure 2.8). This provides, in the case the HADES drift cells, a gas amplification factor of $2 \cdot 10^{5}$ to $3 \cdot 10^{5}$ [Zen97]. Drift velocities in this amplification region are with $150 \mathrm{~cm} / \mu \mathrm{s}$ much faster than in the drift region.

Detailed further information related to the properties and characteristics of the HADES drift cells can be found in [Mar05] .

The minimum distance of a charged particle track to the sense wire can be determined by measuring the drift time of the electrons relative to an external time reference (Trigger) with, in 
case of the HADES MDC detector, a cell dependent resolution ranging from $100 \mu \mathrm{m}$ to $130 \mu \mathrm{m}$. The efficiency of a HADES MDC drift cell is in nearly all cases significantly exceeding $95 \%$ [Mar05]. Left-right ambiguities in a drift cell with only one sense wire as described here are resolved by combining the signals of several drift cells from different layers of the detector. This method also has to be used to reconstruct a track and the individual applied techniques are briefly summarized in Section 2.11.

\subsection{Magnet System - ILSE}

The curvature of charged particle tracks, which enables momentum measurements, is provided in HADES by a superconducting magnet called ILSE (Iron-Less Superconducting Electron Magnet). As visible in Figure 2.1 it follows the sixfold symmetry of the whole setup and is divided into six identical individual aluminum case modules, each containing a cooled superconducting coil, fixed in position by a stable frame, which is also taking the magnetic forces acting between the coils. The field is nearly toroidal, which means in practice that the deflection is mostly in polar angle $\vartheta$. The maximum field strength is of about $B_{\max }=3,7 \mathrm{~T}$ inside the coils, but only of $B \simeq 0,7 \mathrm{~T}$ in the HADES acceptance. The whole design is optimized to keep the other detectors MDC, TOF and RICH as well as the target region free of a magnetic field.

A particle of momentum $\vec{p}$, velocity $\vec{v}$ and charge $q$ in a magnetic field $\vec{B}$ experiencing the LorENTZ-force $\vec{F}$ accumulates an integral change in momentum $\overrightarrow{\Delta p}$ over its path $\mathrm{d} \vec{s}$ given by:

$$
\Delta \vec{p}=\int \mathrm{d} \vec{p}=\int \vec{F} \mathrm{~d} t=\int q(\vec{v} \times \vec{B}) \mathrm{d} t=-q \int \vec{B} \times \mathrm{d} \vec{s}
$$

Since the magnetic field is confined into a small volume, the interaction of the field with the charged particle can be described in a simplified way by a plane in space located in the magnet volume which changes the momentum of the passing charged particle instantly by a momentum "kick" $\Delta \vec{p}$. When appropriately chosen there is no visible difference between this method and a description of the continuous bending of the tracks in the field of the magnet for observes (i.e. detectors) outside the field region. This plane is referred to as kick-plane and the method as kick-plane method (compare Figure 2.2).

The momentum kick ranges from $p_{T}=50 \mathrm{MeV} / \mathrm{c}$ at large polar angles to $p_{T}=100 \mathrm{MeV} / \mathrm{c}$ at small polar angles, while the relative deflection has almost a constant value of $\Delta p / p \simeq 15 \%$. These and further details of the properties of the HADES magnet are available in [Bre99].

\subsection{Time of Flight Detector - TOF}

A Time of Flight (TOF) detector measures the time difference between two detectors detecting a particle. Assuming a given path length, e.g. from the target to the TOF detector, the velocity of the particle can be calculated. In addition, in the case of the HADES TOF, the position of impact and the energy loss in the material can be observed.

The HADES TOF detector [Ago02] covers the polar angles in the range $44^{\circ} \leq \vartheta \leq 88^{\circ}$ per sector and is the outermost detector at these polar angles, as shown in Figure 2.1 and Figure 2.2. It is built from scintillating rods made of $\mathrm{BC} 408$ from the vendor Bicron (scintillation efficiency $\approx 10^{4}$ photons $/ \mathrm{MeV}$; decay time $\left.=2,1 \mathrm{~ns} ; v_{\text {group }} \simeq 16 \mathrm{~cm} / \mathrm{ns}\right)$. Per sector, the rods are organized into eight modules with 8 rods each, for a total of 64 rods per sector and 384 in total. 
The rods emit scintillation light when a particle loses energy in them by interacting with the rod's material. The relevant process here, for all particles but electrons and positrons, is the Bethe-Bloch mechanism described by Equation (2.3) in Section 2.4.1. The scintillation light

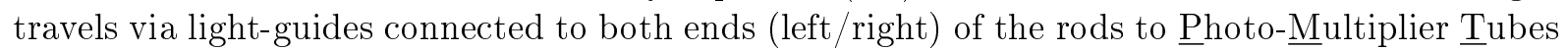
(PMT) where it is amplified, read out and digitized. The position of the particle traversing the rod is measured by calculating the difference of the arrival time of the light on the right end of the bar with the arrival time on the left end of the bar. The resolution is given by the resolution of the time measurement $(\approx 150 \mathrm{ps})$, resulting in a spacial resolution of approximately $2,4 \mathrm{~cm}$. The total efficiency of the TOF detector has been measured to be $\epsilon \approx 0,96$. In addition, this detector is used to identify electrons, since these are (because of their low mass) the only particles in the relevant energy regime with a velocity close to $\beta \simeq 1$.

\subsection{Time of Flight Detector - TOFino}

The TOFino detector covers the polar angle range of $18^{\circ} \leq \vartheta \leq 50^{\circ}$, and it is mounted directly on top of the Pre-Shower detector (Section 2.8 and Figure 2.9), as shown in Figure 2.1 and Figure 2.2. The naming of the detector is an allusion to its small size compared to the TOF detector (based on the Italian language). TOFino serves a similar purpose as the TOF detector (compare Section 2.6), but it has some limitations given by its design: Every sector is covered by only four scintillator plates pointing towards the beam axis, which are read out at one end only. This strongly limits the spacial resolution and multi hit capabilities, making the system useful only for low multiplicity experiments like $\mathrm{p}+\mathrm{p}$ or $\mathrm{C}+\mathrm{C}$. Heavier systems like $\mathrm{Au}+\mathrm{Au}$ would result in too high occupancies. It is foreseen to overcome these restrictions by replacing the TOFino detector with a RPC type detector as described in Section 2.9.

\subsection{Pre-Shower Detector}

The Pre-Shower detector [Bal04] of HADES covers the polar angle region between $18^{\circ}$ and $45^{\circ}$. It is the outermost detector, located directly behind the TOFino detector (Section 2.7), as visible in Figure 2.1 and Figure 2.2. Its operational principle is, as the name implies, the generation of electromagnetic showers (em-showers) by high energy electrons (or positrons) depositing energy in the detector. Electrons (or positrons) passing through matter dominantly lose their energy by Bremsstrahlung. In case of a high incident energy the Bremsstrahlung photons undergo pair production and generate high energy $\mathrm{e}^{+} \mathrm{e}^{-}$-pairs, which again might loose energy via Bremsstrahlung. The measurement of a em-shower indicates an electron traversing the detector, since heavier particles do not produce this kind of shower. This detector is named Pre-Shower, because not the complete shower is observed, but only its first spacial part. This saves a lot of material for the detector, and thus a lot of resources (i.e. money).

The HADES Pre-Shower is built as six modules following the general symmetry of the setup. Each module is built as shown in Figure 2.9. Also shown there is how a shower develops in the lead converters, which have a thickness of two radiation lengths for electrons. This ensures a high probability that electrons initiate a shower, while hadronic showers are not very probable. The multi-wire gas chambers used for the readout operate in SQS mode (Self-Quenching-Streamer), in which incident ionizing particles induce approximately the same amount of charge in the detector, independent of their energy. 


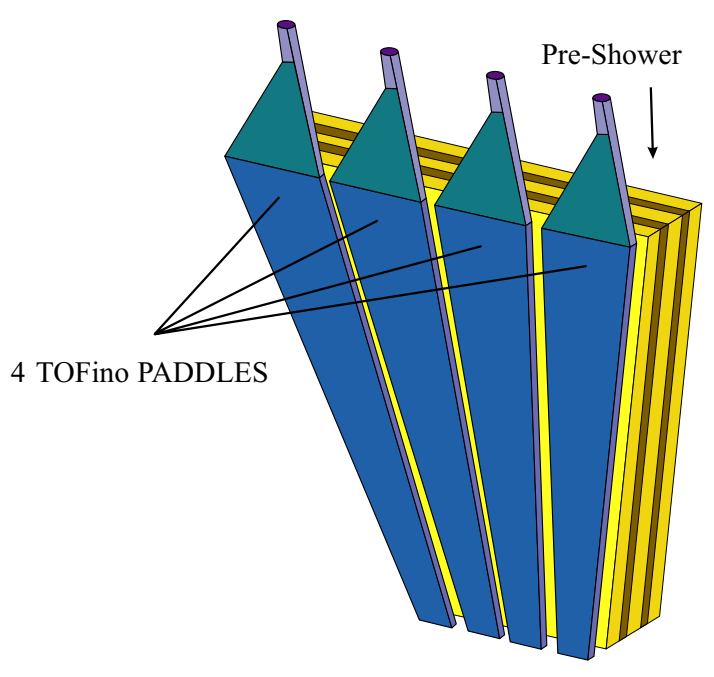

(a) Pre-Shower and TOFino Paddels

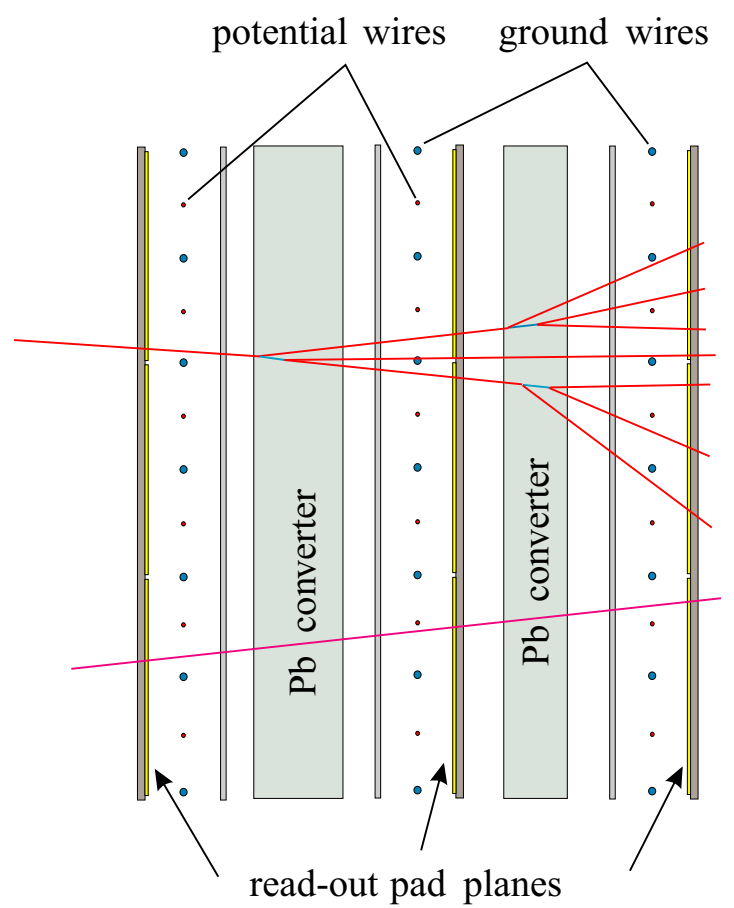

(b) Layers of the Pre-Shower

Figure 2.9.: The Pre-Shower Detector and TOFino Detector [Bal04].

Figure a) shows the shape of the TOFino scintillator paddles in one sector. The readout is connected at the outer end (up). The TOFino is mounted directly in front of the Pre-Shower detector. The layer stacking of the three gaseous detector planes with two interposed $\mathrm{Pb}$ converters is visible in b). Two particles traversing the detector are also shown, one developing a electromagnetic shower (upper) in the converters (i.e. it is a electron or positron), while the other (lower) travels through without developing a shower (for example a pion).

The signals of the different planes are used to find the local maximum in the charge distribution. Around this maximum an integration over $3 \times 3$ pads is performed per layer. Comparing the ratio of these integrated charges per layer against thresholds, it is possible to discriminate electrons and positrons from hadrons. This is especially important since fast small polar angle pions are indistinguishable by their velocity (measured by flight time) via the TOFino detector. The position resolution is better than the detector granularity, which is given by the size of pads of the readout plane (ranging from $2 \mathrm{~cm}$ to $5 \mathrm{~cm}$ ).

\subsection{Resistive Plate Chambers Detector - RPC}

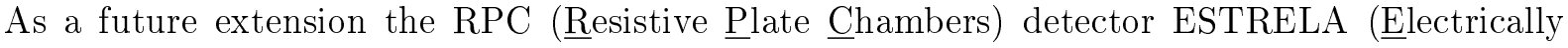

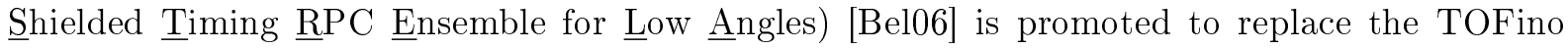
detector as time of flight system at small polar angles. A RPC detector is built from stacks of isolator plates coated with a material of a high resistivity, for example graphite paint, and a gas in 
between. The coating of the plates is held at high voltage, for example $7 \mathrm{kV}$. A charged particle traveling through the gas gap will produce a local avalanche discharge through the gas gap, which is localized and quenched by the fact that the local voltage breaks down due to the high resistivity of the plate's coating. A position sensitive readout can be realized by adding readout strips on the plates in a two dimensional grid. The avalanche discharge couples capacitively into the strips. Since the avalanche develops fast, a detector with precise timing can be realized. The RPC prototype for HADES was tested in beam and showed a timing resolution of $75 \mathrm{ps}$ [Alv04, Bla03].

\subsection{META System}

The META (ultiplicity Electron Trigger Array) system is not a single detector by itself, but the combination of the time of flight systems TOF and TOFino, and the Pre-Shower detector, all described individually in Section 2.6, Section 2.7 and Section 2.8. Together these detectors cover the polar angles in the range $18^{\circ} \leq \vartheta \leq 85^{\circ}$. The data provided is the position of a particle hit (TOF /Pre-Shower) and a discrimination of particle type. Electrons and positrons are identified by TOF by their velocity. Fast pions at small $\vartheta$-angle have velocities too high to be distinguished from electrons in the TOFino detector, but the Pre-Shower detector provides identification by the observation of the development of an electromagnetic cascade (em-shower). The collection of this information is referred to as a hit in META.

\subsection{Tracking in the Magnetic Spectrometer}

Tracks from charged particles are reconstructed in HADES by combining the drift time information of all MDC cells of one sector in several steps. The basic idea behind can be presented in the following way: visualizing MDC I in sector one as seen from the target position (compare Figure 2.1 and Figure 2.2) it looks similar to Figure 2.7 (a). If one imagines that a charged particle emitted from the target position is passing through this module in a straight path, it is clear that it passes different drift cells in different layers of the chamber. There, it is producing with high probability ( $>95 \%$, compare Section 2.4.1) a signal in each layer. Visualizing now every drift cell that has produced a signal (hit cell) as a line, it is obvious that the particle passed through the MDC chamber at the point where all the wires from different layers with their different inclinations cross. The straight lines representing example wires from the different layers in Figure 2.7 demonstrate this. In this picture the particle would have crossed the chamber just in the middle, and would have fired cell (hit, created a signal) in all layers of the module. This way of searching for a track is feasible because the magnetic field (in good approximation) does not penetrate the volume before and inside the MDC chambers. Thus, straight particle tracks are a reasonable assumption except for the actual magnet region.

The full method [Re100, Rus06, Aga00, Aga01, Aga06b] is more elaborate, but follows the basic idea. The track candidate search is performed for two chambers per sector simultaneously. The inner two chambers (MDC I and MDC II) and the outer two chambers (MDC III and MDC IV) are combined into a twelve layer system each. The sensitive volume of each drift cell is projected as a sensitive area onto a plane located between the chambers, as shown in Figure 2.10 for the inner MDC chambers and in Figure 2.11 for the whole setup. The projection is done by drawing a straight line from the boundary of the target volume through the boundary of each drift cell. 


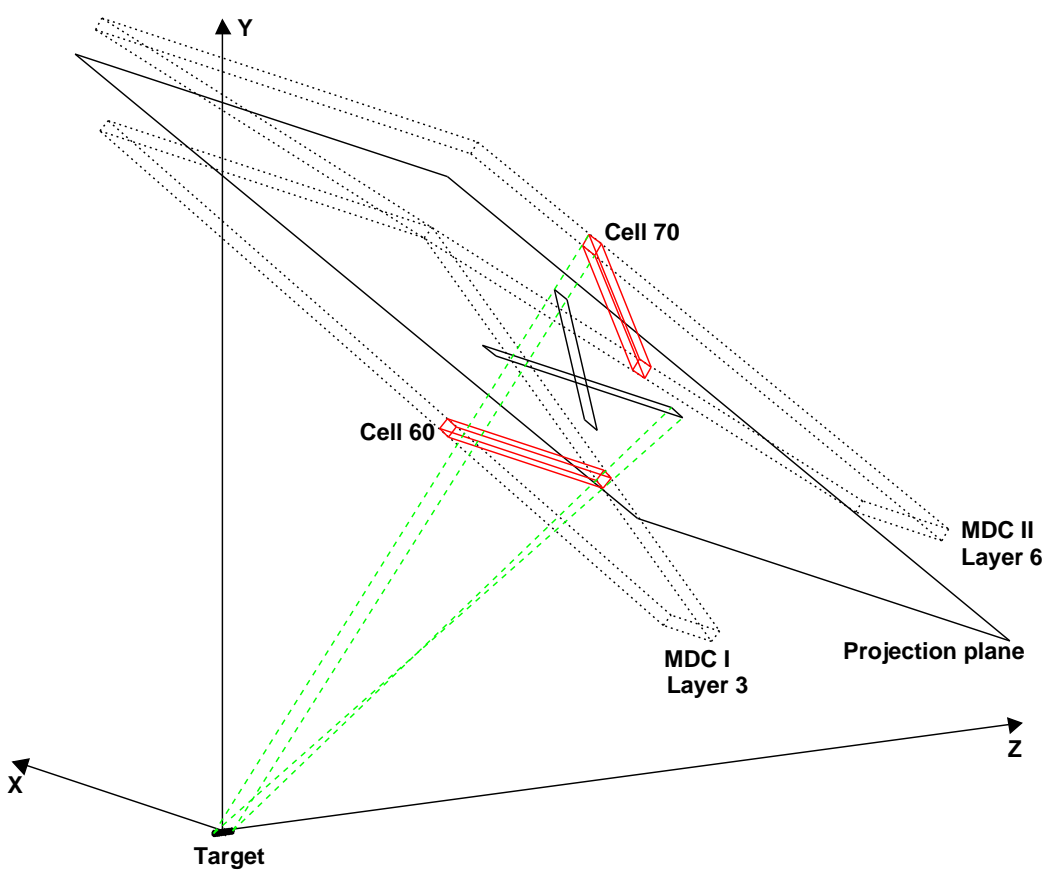

Figure 2.10.: Inner Projection Planes [Aga01, Rus06].

The creation of the projection areas representing the sensitive volume of cell 60 from MDC I layer 3 and MDC II layer 70 is visualized in this figure by drawing straight lines from the outer edges of the target volume to through these cells. While this is shown here only for the inner MDC chambers, Figure 2.11 also shows the procedure for the outer MDC chambers. The figure is not up to scale and the $\mathrm{z}$-axis is expanded.

As shown in the figures, this gives approximately rectangular shaped areas in the projection plane. The position of the projection plane is chosen to equalize the areas of the projections of the sensitive volume form MDC I and MDC II to approximately the same size. These areas can overlap for different cells. The projection plane is then treated as a two dimensional plot with the projection areas as bins, and for each drift cell that gave a signal the bin content is incremented by one (but not more than once per contributing layer), as shown in Figure 2.12 in an example for four particles. By finding the local maximum in this plot, shown magnified in the same figure, track candidates (so called clusters) are found. The used threshold of the local maximum bin content to identify a track candidate depends on the average multiplicity of the reaction under study, but is at least $H_{\min }=4$, so at least four different wires of different planes have to have seen a signal. The track candidates are further processed by analyzing the drift time information of the contributing drift cells. In this way the real tracks with precise spacial information are reconstructed. This procedure is referred to as track fit.

The vertex (interaction point) reconstruction [SG03b] is performed by finding the point in space which is closest to the all reconstructed tracks in one event. Tracks far from a group of other tracks are left out in the fitting procedure. 


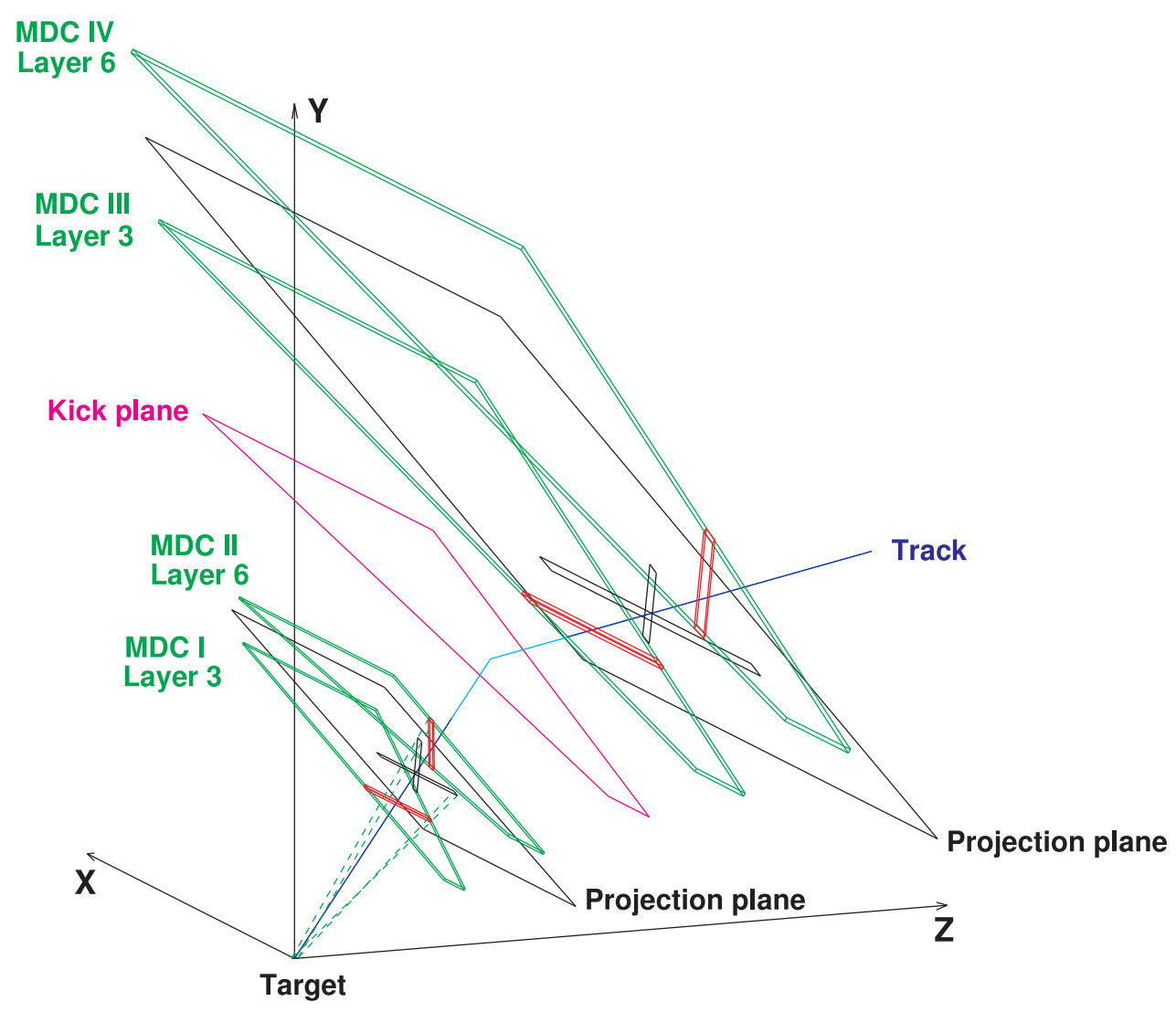

Figure 2.11.: Position of the Kick-Plane and Projection Planes [Aga01, Rus06].

This figure shows the creation of the projection areas representing the sensitive volumes the MDC drift cells for a whole sector. In addition, the kick-plane (compare Section 2.5) is visible in the middle, separating the inner and the outer region of the detector. It's idealized momentum kick on a charged particle can also be seen. The used coordinate system is consistent with the definition in Figure 2.1. The figure is not up to scale and the z-axis is expanded.

The track candidate search for the outer chambers (MDC III and MDC IV) is performed in a similar way. It differs in the position of the projection plane, which is also located between the chambers but is parallel to both in contrast to the inner chambers. It also differs in the point in space from which the straight lines originate, which define the projected areas of the sensitive volumes on the projection plane. The chosen point is where the extrapolation of the track from the inner MDC chambers crosses the kick-plane (described in Section 2.5) of the magnet as shown in Figure 2.11, taking into account its position resolution. This method for the outer MDC chambers is repeated for every track calculated from the data of the inner MDC chambers. As for the inner chambers the drift time information is then used to get the full spacial reconstruction.

The momentum reconstruction can be performed with the so called kick-plane method described in detail in [SG03b] and outlined in Section 2.5, or by two more advanced techniques, the so called Spline method and the so called Runge-KutTa method, both described in [Rus06]. 

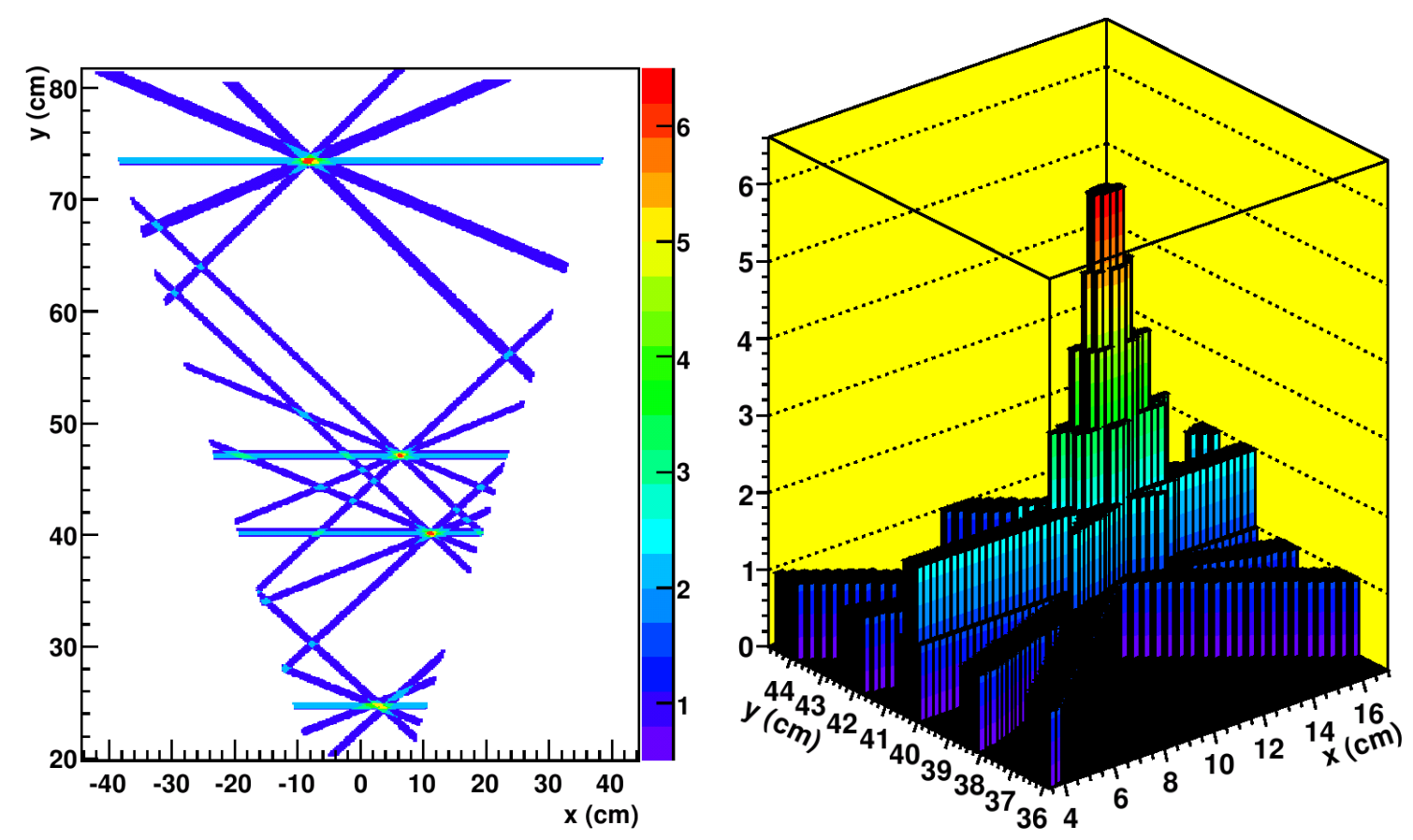

Figure 2.12.: Track Candidate Search (cluster finder) [Aga01, Rus06].

The bins representing the projections of the sensitive volumes of the MDC drift cells are incremented by one (z-axis) for each fired cell they represent, limited to a maximum of one per contributing layer. At places where a certain height is reached (e.g. $H_{\min }=4$ ) a cluster can be found, most likely caused by a particle passing through this point of the projection plane. The right figure shows a magnified $3 \mathrm{D}$ view of one of the clusters.

\subsection{The Trigger System}

The use of high primary beam intensities of up to $10^{8}$ particles/s and a $1 \%$ interaction length target yield reaction rates of up to $10^{6} \mathrm{~Hz}$, which call for a sophisticated real time trigger system, to enrich the data sample with the rare decays of light vector mesons into dileptons. For example, in a central $\mathrm{Au}+\mathrm{Au}$ collision at $1 \mathrm{AGeV}$ the rate of dileptons from $\rho$-decays is only $10^{-6}$ pairs/event.

The HADES trigger system [Toi03] is structured in several levels and implemented in different hardware modules, as shown and summarized in Figure 2.13. The decision if there was a reaction inside the target region is taken by the START/VETO as described in Section 2.2. The multiplicity signature of the event is the first level trigger (LVL1). The second level trigger (LVL2) selects events which show characteristic lepton signatures. Trigger levels up to LVL2 are implemented in dedicated hardware modules. Data reduction beyond the second level trigger is presently part of the software framework and performed offline. 


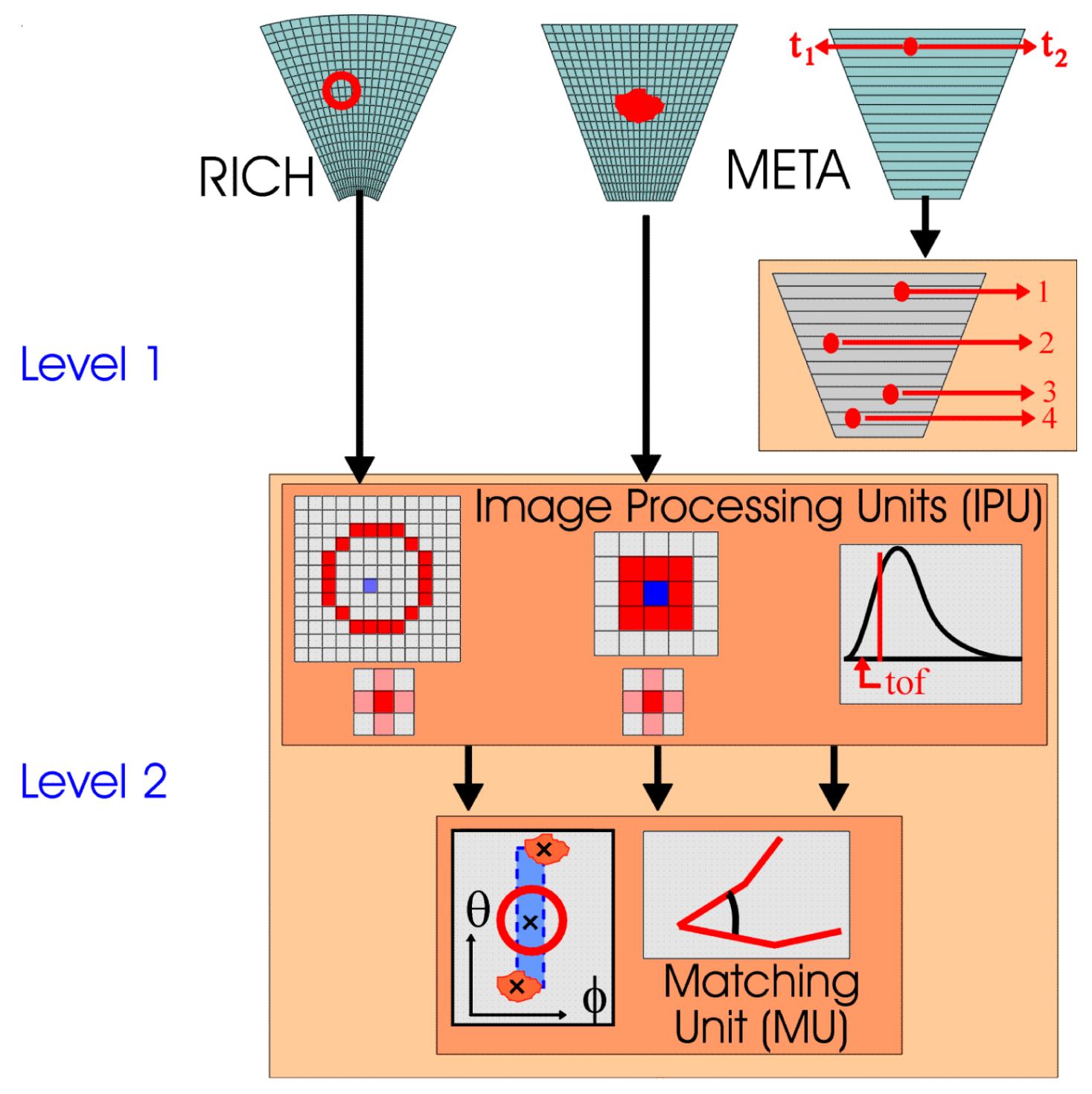

Figure 2.13.: The HADES Trigger System [Leh99].

The top row outlines the detectors which deliver data for the Trigger System: RICH (fired pads), Pre-Shower (fired pad with pulse height) and TOF (TDC (Time to Digital Converter) signals). The hit multiplicity from META (TOF) is used for the LVL1 trigger decision. The data is further

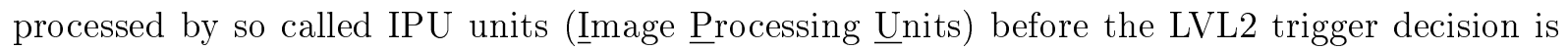
taken by the MU (ㅍatching $\underline{\text { Unit)}}$.

An IPU is a dedicated hardware system processing the data of one detector system, extracting the relevant information for the LVL2 trigger: The TOF IPU [Lin01, Frö02] provides the time of flight, The SHOWER IPU [Pet00] provides the position of an em-shower and the RICH IPU [Leh00] provides the position of rings (see also Figure 2.6). All modules deliver their information in real-time to the Matching Unit [Tra01], where it is correlated to form the LVL2 trigger decision. It should be noted that no information from the tracking chambers (MDC) is available for the online trigger system. 


\subsubsection{The First Level Trigger - LVL1}

The First Level Trigger (LVL1) is a multiplicity trigger. The number of hits in TOF and TOFino is compared to given settings, and a trigger decision is delivered in approximately $100 \mathrm{~ns}$ to $200 \mathrm{~ns}$. This task is performed by the MTU (uultiplicity Trigger $\underline{\text { Unit}})$. For heavy systems like $\mathrm{Au}+\mathrm{Au}$ the selection of a certain minimum multiplicity translates directly into the selection of events with a certain minimum impact parameter. A proper impact parameter selection like $b<4 \mathrm{fm}$ yields reduction factors in event rate in the order of 10 [Leh00, Tra01].

\subsubsection{The Second Level Trigger - LVL2}

The Second Level Trigger (LVL2) decision is taken by a dedicated hardware module called the Matching $\underline{\text { Unit }}$ (MU) [Tra01]. Its operational principle is shown in Figure 2.13. It operates by correlating the angular information obtained from the RICH IPU with the angular information from the TOF IPU and SHOWER IPU (META) per sector. The angular information is sufficient to calculate the momentum of the particle based on the kick-plane approach. Events containing a signature of a particle that is consistent with an electron or a positron are accepted. Detailed studies of the behavior of this subsystem, with different settings and parameters, have been performed by [Tra01, Toi04]. For example, the achievable reduction rate is, as it was demonstrated during the November 2002 beam-time, in the order of a factor of 12, if the matching unit is set up to require at least one lepton in a matching window of $\Delta \phi=9^{\circ}$. The precise TOF, SHOWER and RICH settings used can be found in the references.

\subsubsection{Higher Trigger Levels (LVL3) and new Challenges}

The selection of events beyond the trigger levels described in the previous sections is currently implemented in the offline analysis. The implementation of higher trigger level (LVL3) in hardware could significantly reduce the load on the DAQ system and the offline analysis. Both loads will steadily increase as more heavy collision systems are being studied. While it has been shown by the two $\mathrm{C}+\mathrm{C}$ beamtimes that the current trigger system is working adequately for systems of this size, the heaviest system measured so far $(\mathrm{Ar}+\mathrm{KCl})$ showed that the current trigger system is reaching its limits.

When changing towards heavier reaction systems like $\mathrm{Ar}+\mathrm{KCl}$ the current trigger system is no longer sufficient for several reasons. The most general reason is the high detector occupancy. The evolution of the detector occupancies and the resulting much larger data samples per events (as far as relevant for the later described topics) are listed in Table 2.3. In addition to the higher data rates introduced by the higher occupancies several other effects play an important role, and are getting more pronounced as the systems become heavier.

First, the direct ionization noise in the RICH MWPC readout pads caused by charged particles is increasing with the event multiplicity. This is even more severe as the analog MWPC data samples are not used by the RICH IPU to suppress the detection of rings formed by this noise. This is one reason of the strong fake contamination of the RICH IPU rings.

The second issue is the absence of the MDC detector in the trigger system. Its presence could strongly help to improve the lepton identification. For example, a lot of the RICH IPU rings are not correlated with any detector response consistent with any kind of track in the MDC chambers. 
Another problem, also growing with the multiplicities, is the occupancy of the TOFino detector. The granularity of this detector is very low (compare Section 2.7). This means in practice for heavy systems, that HADES is having problems in distinguishing between the many particles traveling at low polar angles.

Summarized, in larger systems like $\mathrm{Ar}+\mathrm{KCl}$ the detector occupancy reaches values where the second level trigger does not provide anymore an enhancement in the order of 10 . Instead, it is in the order of 2-3 and will drop further as more heavy systems are studied.

These problems are currently being addressed in several ways. One is to replace the TOFino detector with the RPC system described in Section 2.9, thus solving the granularity problem. Another foreseen improvement is the replacement of the RICH IPU algorithm with a more advanced version which does use the analog information of the RICH readout, and implements a more elaborate way to find rings than the pure bit-pattern matching of the current implementation (compare Figure 2.6).

In addition, the shortcoming of the current trigger system of not being able to use any data from the MDC chambers will be solved with the trigger hardware upgrades described in detail in Chapter 5. This makes advanced trigger algorithms feasible, like the one which is described and analyzed in Chapter 4 as one major part of this thesis. This method is correlating the RICH IPU ring data with the fired wires of the MDC chambers to trigger on events which contain real electrons (no fakes) and could be operated as a third level trigger (LVL3).

A hardware extension, the GE-MN (Gigabit Ethernet - $\underline{\text { Multi }}$ Node), is presented in Chapter 3. In short, this system provided the test and development environment needed for the implementation of the RICH to MDC correlation trigger mentioned above in a realistic test system. Further, it enables the evaluation of a standard network interconnect architecture based custom built hardware module in real-time trigger applications.

Table 2.3.: Average Detector Occupancy and Average Raw Data per Event.

The average RICH and inner MDC detector occupancy per event is listed in the upper table. The relevant quantity for the MDC detector is the number of fired drift cells (TDC channels), or, in case of the RICH detector, the number of fired MWPC pads. For reference, the average number of rings found by the RICH IPU is also stated.

The lower table lists the resulting average raw data sizes per event for the RICH and the MDC detector. In addition, the average size of the full event data set is shown. The raw data sizes are only the pure detector data, while the average event sizes contains also several headers [Kol98].

\begin{tabular}{|c||c|c|c|}
\hline $\begin{array}{c}\text { Experiment } \\
\text { Reaction @ Energy }\end{array}$ & $\begin{array}{c}\text { MDC Occupancy } \\
\text { Fired Cells }\end{array}$ & $\begin{array}{c}\text { RICH Occupancy } \\
\text { Fired Pads }\end{array}$ & $\begin{array}{c}\text { RICH IPU } \\
\text { found Rings }\end{array}$ \\
\hline \hline $\mathrm{p}+\mathrm{p} @ 2,2 \mathrm{GeV}$ & 41 & 47 & 0,65 \\
\hline $\mathrm{C}+\mathrm{C} @ 1 \mathrm{AGeV}$ & 90 & 67 & 0,28 \\
\hline $\mathrm{Ar}+\mathrm{KCl} @ 1,75 \mathrm{AGeV}$ & 355 & 116 & 1,5 \\
\hline
\end{tabular}

\begin{tabular}{|c||c|c|c|}
\hline $\begin{array}{c}\text { Experiment } \\
\text { Reaction @ Energy }\end{array}$ & $\begin{array}{c}\text { MDC Raw Data } \\
\text { in Byte }\end{array}$ & $\begin{array}{c}\text { RICH Raw Data } \\
\text { in Byte }\end{array}$ & $\begin{array}{c}\text { Event Size } \\
\text { in KByte }\end{array}$ \\
\hline \hline $\mathrm{p}+\mathrm{p} @ 2,2 \mathrm{GeV}$ & 165 & 188 & 2,6 \\
\hline $\mathrm{C}+\mathrm{C} @ 1 \mathrm{AGeV}$ & 359 & 268 & 7,2 \\
\hline $\mathrm{Ar}+\mathrm{KCl} @ 1,75 \mathrm{AGeV}$ & 1418 & 464 & 31,8 \\
\hline
\end{tabular}




\subsection{Bibliography}

[Aga00] G. N. Agakichiev; V. N. Pechenov; Yu. V. Zanevsky et al. (HADES); Fast method for searching tracks in Multiwire Drift Chambers of HADES spectrometer (April 2000); internal HADES manual

[Aga01] G. N. Agakichiev and V. N. Pechenov (HADES); Dubna tracks reconstruction user manual (December 2001); HADES internal manual

[Aga06b] G. N. AgAKICHIEv and V. N. Pechenov (HADES); private communication (November 2006)

[Ago02] C. Agodi; A. BAssi; R. Bassini et al. (HADES); The HADES time-of-flight wall; Nuclear Instruments and Methods in Physics Research A (2002); 492:14-25

[Alv04] H. Alvarez Pol; R. Alves; A. Blanco et al. (HADES); A large area timing RPC prototype for ion collisions in the HADES spectrometer; Nuclear Instruments and Methods in Physics Research A (2004); 535:277-282; doi:10.1016/j.nima.2004.07.276

[Bal04] A. BAlANDA et al. (HADES); The HADES Pre-Shower detector; Nuclear Instruments and Methods in Physics Research A (2004); 531:445-458

[Bel06] D. BELver et al. (HADES); The Front-End Electronics for the HADES RPC Wall (ESTRELA-FEE); Nuclear Physics B (Proc. Suppl.) (2006); 158:47-51

[Ber99] E. Berdermann; K. Blasche; P. Moritz et al. (HADES); First Applications of CVD-Diamond Detectors in Heavy-ion Experiments; Nuclear Physics B (Proc. Suppl.) (1999); 78:533-539

[Bla03] A. Blanco; R. Ferreira-Marques; Ch. FincK et al. (HADES); Single-gap timing $R P C s$ with bidimensional position-sensitive readout for very accurate TOF systems; Nuclear Instruments and Methods in Physics Research A (2003); 508:70-74

[Böh99] M. BöHMER (HADES); Das Auslesesystem für den Ringabbildenden Cherenkovdetektor im HADES Spektrometer (1999); Physik-Department der Technischen Universität München, Institut E12

[Bre99] Thomas Bretz (HADES); Magnetfeldeigenschaften des Spektrometers HADES (1999); Diplomarbeit, Technische Universität München

[Čer37b] Pavel Alekseyevich Čerenkov; Visible Radiation Produced by Electrons Moving in a Medium with Velocities Exceeding that of Light; Physical Review Letters (Aug 1937); 52(4):378-379; doi:10.1103/PhysRev.52.378

[Fab03a] Laura FabBietTi (HADES); Study of the $e^{+} e^{-}$pair acceptance in the dilepton spectrometer HADES; Ph.D. thesis; Technische Universität München (2003)

[Fab03b] Laura Fabbietti; T. Eberl; J. Friese et al. (HADES); Photon detection efficiency in the CsI based HADES RICH; Nuclear Instruments and Methods in Physics Research A (2003); 502:256-260 
[Fri03] J. Friese; R. Gernhäuser; P. Maier-Komor et al. (HADES); A new carbon based VUV mirror of righ radiation lenght for the HADES RICH; Nuclear Instruments and Methods in Physics Research A (2003); 502:241-245

[Frö02] INGo FrÖHLICH (HADES); Untersuchungen zur $\eta$-Produktion in pp-Reaktionen mit dem DISTO- und HADES-Spektrometer; Ph.D. thesis; II. Physikalisches Institut der Justus-Liebig-Universität Gießen (February 2002); URL http://pcweb.physik. uni-giessen.de/exp2

[Gar98] C. Garabatos et al. (HADES); Optimisation of low-mass drift chambers for HADES; Nuclear Instruments and Methods in Physics Research A (1998); 412:38-46

[HAD94] HADES Collaboration; A proposal for a High Acceptance Di-Electron Spectrometer (1994); GSI Darmstadt

[Jac99] John David Jackson; Classical Electrodynamics (John Wiley \& Sons, Inc., 1999); 3rd edition; ISBN: 0-471-30932-X

[Kol98] B. KolB et al.; HADES raw data event lengths (April 1998); see also: http://www-hades.gsi.de/docs/mdc/mdcana/mdc-essentials.htm; URL http:// www-hades.gsi.de/daq/event_lengths.html

[Leh99] Jörg LehnerT; Rene Becker; Ingo Fröhlich et al. (HADES); Ring recognition in the HADES second-level trigger; Nuclear Instruments and Methods in Physics Research A (1999); 433:268-273

[Leh00] JÖRG LEHNERT (HADES); Echtzeit-Mustererkennung zum Elektronennachweis mit einem RICH-Detektor in relativistischen Schwerionenkollisionen; Ph.D. thesis; II. Physikalisches Institut der Justus-Liebig-Universität Gießen (June 2000); URL http: //pcweb.physik.uni-giessen.de/exp2

[Leh03] Jörg Lehnert; Ingo Fröhlich; Wolfgang KÜhn et al. (HADES); Performance of the HADES ring recognition hardware; Nuclear Instruments and Methods in Physics Research A (2003); 502:261-265

[Lin01] ERIK Lins (HADES); Entwicklung eines Auslese- und Triggersystems zur Leptonenidentifizierung mit dem HADES-Flugzeitdetektor; Ph.D. thesis; II. Physikalisches Institut der Justus-Liebig-Universität Gießen (March 2001); URL http://pcweb. physik.uni-giessen.de/exp2

[Mar05] JOCHEN MARKeRT (HADES); Untersuchung zum Ansprechverhalten der VieldrahtDriftkammern niedriger Massenbelegung des HADES Experimentes; Ph.D. thesis; Fachbereich Physik der Johann Wolfgang Goethe-Universität (2005)

[Mün04] C. Müntz; Jochen Markert; G. N. Agakichiev et al. (HADES); The HADES tracking system; Nuclear Instruments and Methods in Physics Research A (2004); 535:242-246 
[Pet00] Markus Petri (HADES); Entwicklung eines kombinierten Auslese- und EchtzeitTriggersystems zum Nachweis von Elektronen/Positronen-Signaturen in einem elektromagnetischen Schauerdetektor; Ph.D. thesis; II. Physikalisches Institut der JustusLiebig-Universität Gießen (August 2000); URL http://pcweb.physik.uni-giessen . de/exp2

[Rel00] Relativistic Nuclear Physics: from Hundreds $\mathrm{MeV}$ to TeV: Proc.of Int. Workshop, Stara Lesna, Slovak Republic; THE SPECTROMETER HADES TRACKING SYSTEM

[Rus06] Anar Rustamov (HADES); Exclusive $\eta$ Meson Reconstruction in Proton-Proton Collisions at $2.2 \mathrm{GeV}$ with the HADES Spectrometer and High Resolution Tracking; Ph.D. thesis; Fachbereich Physik der Technischen Universität Darmstadt (July 2006); URL https://www-new.gsi.de/gsitools

[Sal02] Piotr Salabura (HADES); Vector Mesons in Stronly Interacting Systems (The Marian Smoluchowski Institute of Physics of the Jagellonian University, ul. Reymonta 4, 30-059 Kraków, Poland, 2002)

[Sch95] HEIKE SCHÖN (HADES); HADES Ein Dielektronenspektrometer mit hoher Akzeptanz für relativistische Schwerionenkollisionen; Ph.D. thesis; Johann-Wolfgang-Goethe Universität in Frankfurt am Main, Institut für Kernphysik (1995)

[Sch06] Alexander Schmah (HADES); The HADES Wiki - Pictures and Figures (nov 2006); URL http://hades-wiki.gsi.de

[Seg94] J. Seguinot and T. Ypsilantis; A historical survey of ring imaging Cherenkov counters; Nuclear Instruments and Methods in Physics Research A (1994); 343:1-29

[Seg99] J. Seguinot and T. Ypsilantis; Evolution of the RICH technique; Nuclear Instruments and Methods in Physics Research A (1999); 433:1-16

[SG03b] Manuel SÁncheZ-GarCía (HADES); Momentum reconstruction and pion production analysis in the HADES spectrometer at GSI; Ph.D. thesis; Universidade de Santiago de Compostela (2003)

[Str98] J. Stroth (HADES); A Low-mass Tracking System for HADES; in WCC 98 (1998) URL http://www-hades.gsi.de/mdc/talks/vienna98/vienna98.ppt

[Toi03] Alberica Toia; Ingo Fröhlich; Wolfgang Kühn et al. (HADES); A highly selective dilepton trigger based on ring recognition; Nuclear Instruments and Methods in Physics Research A (2003); 502:270-274

[Toi04] Alberica Toia (HADES); Performance of the HADES Spectrometer for Dilepton Identification in the Reaction $C+C$ at 1 AGeV; Ph.D. thesis; II. Physikalisches Institut der Justus-Liebig-Universität Gießen (March 2004); URL http://pcweb . physik. uni-giessen.de/exp2

[Tra01] Michael Traxler (HADES); Real-Time Dilepton Selection for the HADES Spectrometer; Ph.D. thesis; II. Physikalisches Institut der Justus-Liebig-Universität Gießen (2001); URL http://pcweb.physik.uni-giessen.de/exp2 
[Yao06d] W.-M. Yao et al.; Review of Particle Physics; Journal of Physics G (2006); 33:1-1232; doi:10.1088/0954-3899/33/1/001; URL http://pdg. lbl.gov

[Yao06f] W.-M. Yao et al.; Review of Particle Physics; Journal of Physics G (2006); 33:271292; Particle Detectors; doi:10.1088/0954-3899/33/1/001; URL http://pdg.lbl.gov

[Yao06g] W.-M. Yao et al.; Review of Particle Physics; Journal of Physics G (2006); 33:258-270; Passage of Particles through Matter; doi:10.1088/0954-3899/33/1/001; URL http: $/ /$ pdg.lbl.gov

[Yps94] T. Ypsilantis and J. Seguinot; Theory of ring imaging Cherenkov counters; Nuclear Instruments and Methods in Physics Research A (1994); 343:30-51

[Zei99a] K. Zeitelhack; A. Elhardt; J. Friese et al. (HADES); The HADES RICH detector; Nuclear Instruments and Methods in Physics Research A (1999); 433:201-206

[Zen97] Alfred Zentek (HADES); Konzeptionelle Untersuchungen für die HADESDriftkammern am Prototyp O (1997); Diplomarbeit, Fachbereich Physik der Johann Wolfgang Goethe-Universität 


\section{The GE-MN System}

\section{Contents}

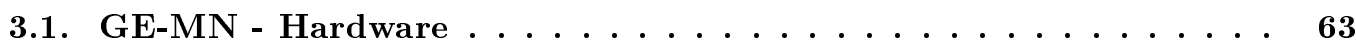

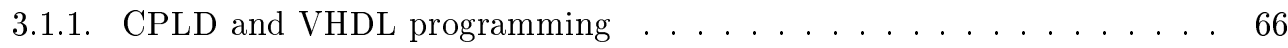

3.1.2. VMEbus Access . . . . . . . . . . . . . . . . . 67

3.1.3. Network Components . . . . . . . . . . . . . . . . 67

3.1.4. Digital Signal Processor . . . . . . . . . . . . . . . . 68

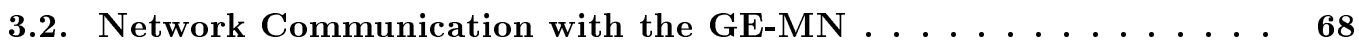

3.2.1. Address Resolution Protocol - ARP . . . . . . . . . . . . . 69

3.2.2. Internet Protocol - IP /ICMP . . . . . . . . . . . . . . 69

3.2.3. User Datagram Protocol - UDP . . . . . . . . . . . . . . . . . . 69

3.2.4. Transmission Control Protocol - TCP . . . . . . . . . . . . . . 70

3.2.5. GE-MN Network Communication Model . . . . . . . . . . . . . . 70

3.2.6. Multi-Node Network Topologies with GE-MN Systems . . . . . . . . . 72

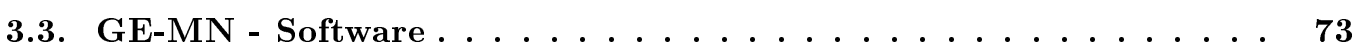

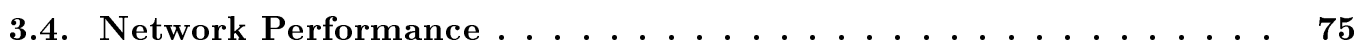

3.4.1. Network Throughput . . . . . . . . . . . . . . . 75

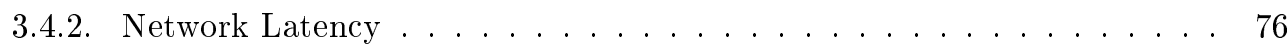

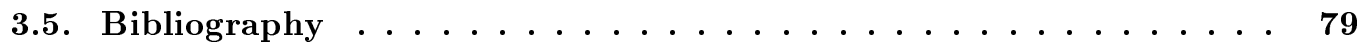

The GE-MN (Gigabit Ethernet - $\underline{\text { Multi }}$ Node) is designed to be a flexible hardware test and development platform to investigate the benefits and challenges of using a high speed commercial Gigabit network technology as data transport interconnect in a custom build embedded system for applications typical for the physics investigated at accelerator facilities using large particle detectors. The following sections describe the GE-MN and its capabilities in general, while Chapter 4 provides insight into the performance of the GE-MN when used in a realistic application in a physics experiment.

\subsection{GE-MN - Hardware}

The system architecture of the GE-MN is shown in Figure 3.1, while the final hardware implementation is shown in Figure 3.2. As shown, the system architecture can be divided into several parts. A network part consisting of a of a pair of DP83861 [Nat01] PHYter ${ }^{1}$ (Gigabit Ethernet [IEE00] over Copper Transceiver, physical layer $\mathrm{ASIC}^{2}$ in green) enabling physical medium access, a PM3386 [PMC01] dual ported MAC (Media Access Controller, green) providing logical medium access and a Delta39K100 [Cyp01] CPLD (omplex Programmable Logic Device, in light blue) providing the interconnect to the DSP.

\footnotetext{
${ }^{1}$ PHYter or PHY: Physical Layer Chip

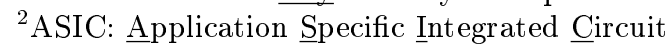




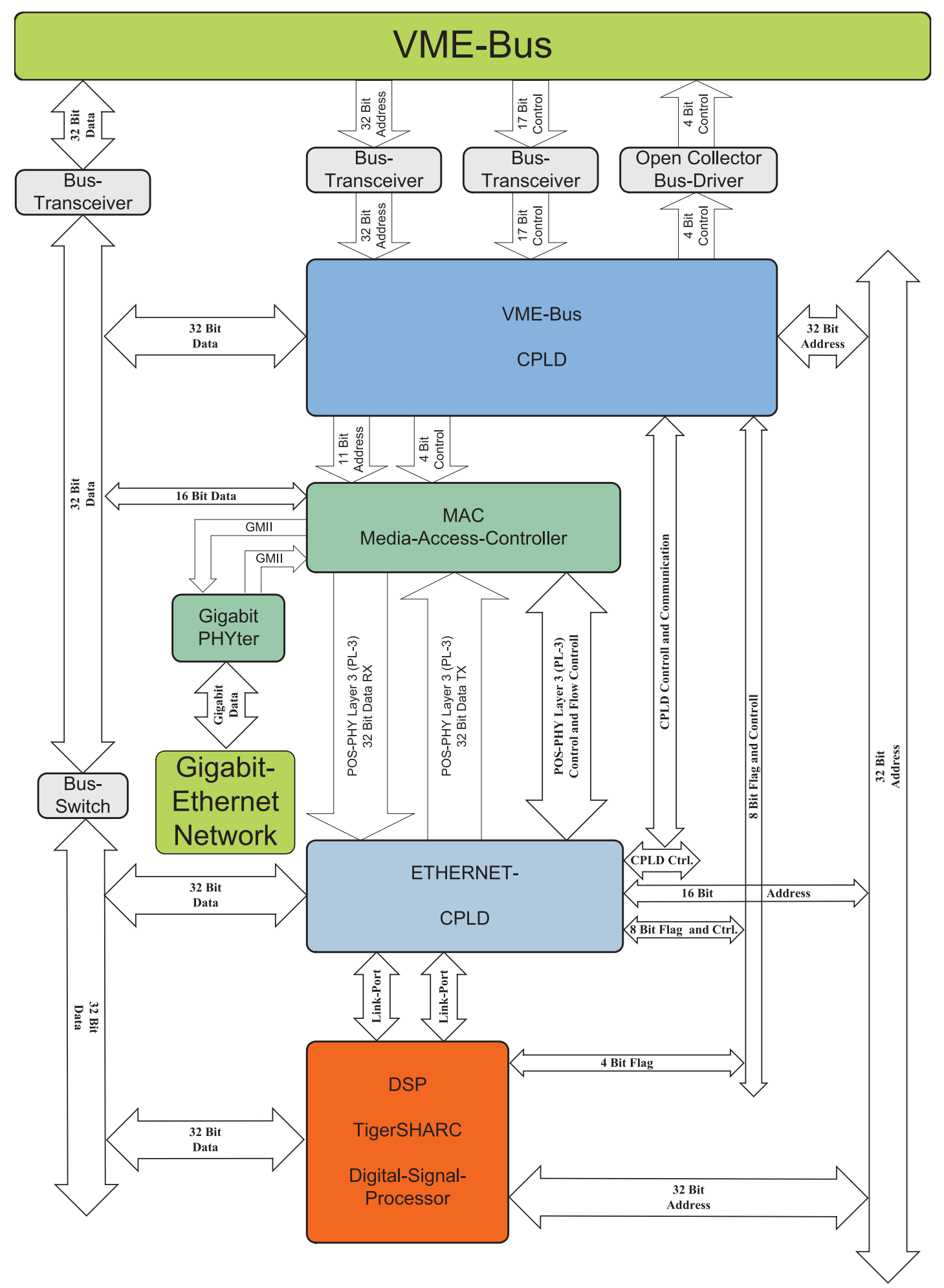

Figure 3.1.: The GE-MN System Architecture.

The external interconnects, the VMEbus and the two Gigabit Ethernet links are in different greens. The red component represents the TigerSHARC DSP as data processing unit. The two CPLD chips, providing general control logic and custom glue logic, are indicated in different blue. Glue-Logic is colored in light gray. Also compare with the real system shown in Figure 3.2 and refer to Section 3.1 for details. 


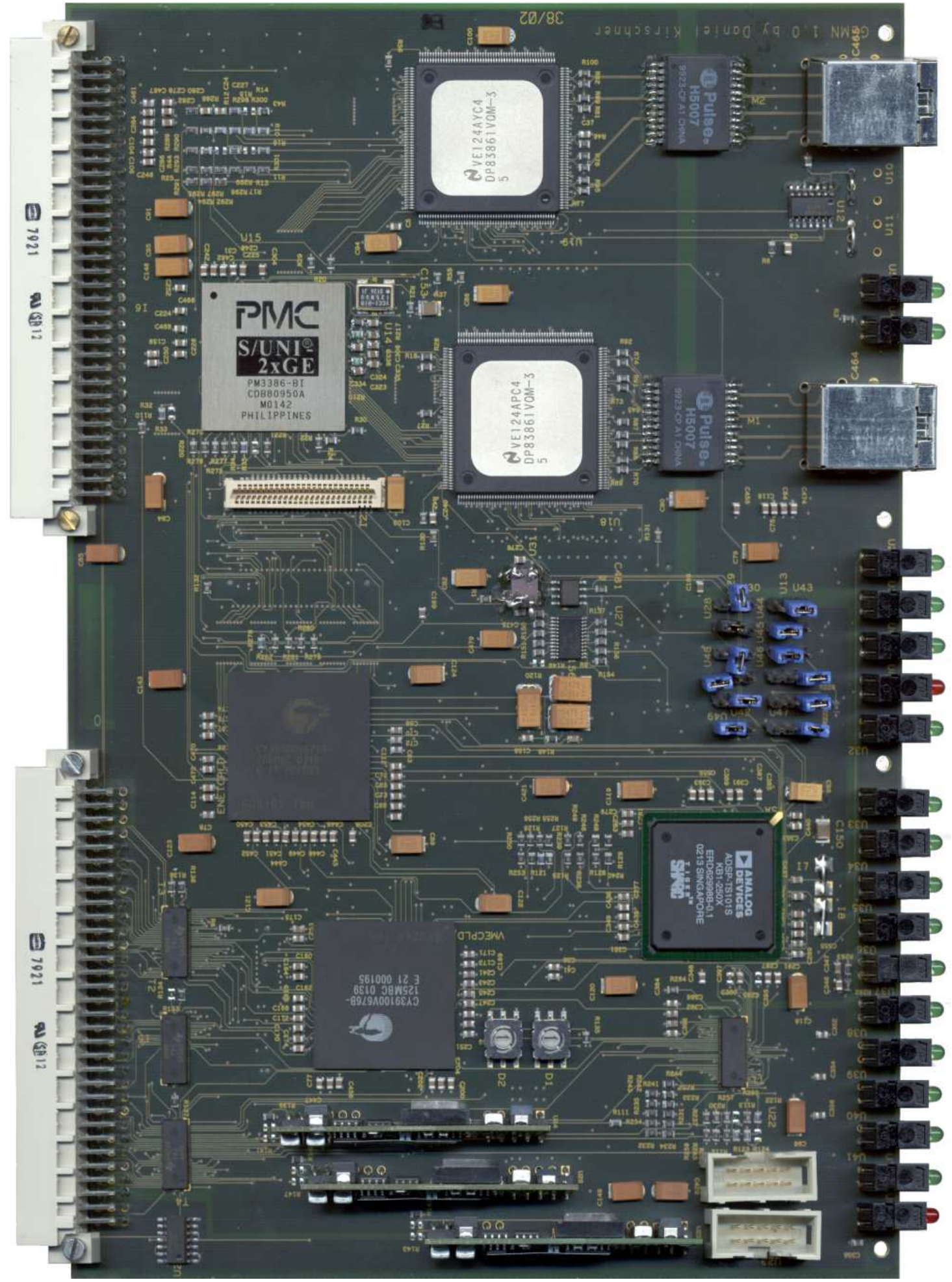

Figure 3.2.: The GE-MN Printed Circuit Board (Top Side).

The left board edge shows the VMEbus connectors, the upper half features the network part consisting of a PM3386 MAC, two DP83861 PHYter, H5007 Magnetics and RJ-45 connectors. The lower half of the PCB features two Delta39K CPLD, a TigerSHARC DSP, DC-DC converters and JTAG ports. For further details refer to Section 3.1 and compare with Figure 3.1. 
The processing subsystem is formed by a TigerSHARC DSP (Digital Signal Processor, in red) [Ana02, Ana04]. An implementation of the VMEbus [VIT06, Pet93] is providing access to general support, control and debug functionality, like resetting individual components, booting the DSP and monitoring registers. This part consists of an other Delta39K100 CPLD (blue) and a collection of bus switches and bus transceivers (light gray).

Data flow and access paths in the system are either via the Ethernet Interfaces or via the VMEbus. The data from and to the Gigabit Ethernet links flows via the GMII ${ }^{3}$ [IEE00] bus and the PL3 (POS-PHY ${ }^{4}$ Layer 3) bus [ATM00, PMC00] via the Ethernet-CPLD and the TigerSHARC Link-Ports [Ana02] to the DSP, independently and undisturbed from other system activity. All of these links are dedicated and the transfers are handled by dedicated hardware units as Direct Memory Access (DMA). The VMEbus is a well established back-plane bus technology used in may physics experiments and provides all basic functionality to power, boot and debug the GEMN, while Ethernet as the most dominant and present network technology in local area networks provides fast remote access for applications.

\subsubsection{CPLD and VHDL programming}

The GE-MN uses two large Cypress Delta39K CPLD chips. The configuration of the CPLD (Complex Programmable Logic Device) devices is performed with the standard language VHDL (VHSIC $^{5}$ Hardware Description Language) using the software tools Synplify Pro [Syn06] and Cypress Warp [Cyp02]. Verification and development of the VHDL modules was performed using the NC-Sim[Cad06] simulator.

The VMEbus CPLD (VME-CPLD) serves as the central place for the control of all basic system functionality. This chip is responsible for performing the reset sequences of all other chips on the board, it controls and provides the Wishbone arbiter and Wishbone interconnect [Her02, Wis06]. It also provides transparent bridges for all the different involved bus systems (TigerSHARC, MPI, VMEbus) from and to the internal Wishbone bus. This is used internally as standard interface bus, to enable communication of all the components across the GE-MN, and most important from the VME-CPU via the VMEbus to all the internal registers and memories inside all components. This data path is also used for most low level debugging, as well as to boot the DSP.

The Ethernet CPLD (ENET-CPLD) is used to enable fast synchronous communication of the TigerSHARC DSP with the Gigabit-Ethernet MAC (see Section 3.1.3). It interfaces one Link Port of the DSP to the TX (transmit) channel of the POS-PHY Layer 3 bus of the MAC, and another Link Port to the RX (receive) channel of the PL3 bus. Comparing the characteristics of these quite different bus systems in Section 3.1.4 and Section 3.1.3, it is clear that a simple one to one mapping is not possible and an elaborate interface had to be developed in VHDL which is operating pipelined and buffered.

\footnotetext{
${ }^{3}$ GMII: Gigabit Media Independent Interface

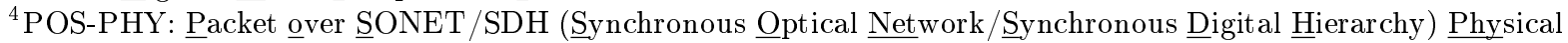
Layer

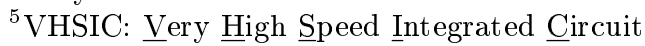




\begin{tabular}{|c|c|c|}
\hline VMEbus Address & Width & Functional Description \\
\hline \hline $31-28$ & 4 & GE-MN module (board) select 0x0-0xF \\
\hline 27 & 1 & TigerSHARC/Chip selector \\
\hline $27-02$ & 25 & effective board internal address space \\
\hline $26-24$ & 3 & Internal component addresses: \\
& & 0xFF = illegal; 0x0C = Wishbone Dummy; \\
& & 0x44 = VME-CPLD; 0x48 = ENET-CPLD \\
& & 0x50 = PM3386 MAC MPI \\
& & 0x60 = unused; 0x80 = unused \\
\hline $23-02$ & 22 & GE-MN internal Address space \\
\hline $1-0$ & 2 & always equal to "00" due to 32 Bit bus granularity \\
\hline
\end{tabular}

Table 3.1.: GE-MN memory map as seen from the VME-CPU via the VMEbus.

Examples Base Addresses: TigerSHARC number 0 on Board 1: 0x18000000; PM3386 MAC on Board 5: 0x55000000; VME-CPLD Registers on Board 2: 0x24400000. The GE-MN module select bits are set by the rotary dip switches visible in Figure 3.2 (lower half, middle), or via the programming of the VME-CPLD. Details of the meaning of the registers can be found in the data sheet of the component (see Bibliography) in question. The Dummy interface is a test module accepting all transfers and answering always with the same pattern.

\subsubsection{VMEbus Access}

The main interface to the GE-MN is the VMEbus System. This classical back-plane bus is used for all basic support, control and debugging accesses. In more detail, it permits to access the address spaces of the different components in the GE-MN (compare Table 3.1). The registers in the VME-CPLD and the ENET-CPLD provide elementary support functions like performing a hardware reset sequence of the chips. In addition, the whole address space of the TigerSHARC DSP can be accessed in parallel and without disturbing a running program (firmware), which provides ideal remote low level monitoring and debugging capabilities of the programs running in the DSP.

For practical reasons the VMEbus implementation in the GE-MN supports accesses according to the A32/D32 (32 bit wide addresses and data) access scheme instead of the original A24/D16 scheme. The number of bus accesses per second is limited to 2000 and block transfers not are supported. This performance is sufficient for the previously described control and debug tasks.

\subsubsection{Network Components}

The network subsystem of the GE-MN uses a PM3386 [PMC01] dual ported Media $\underline{\text { Access }}$ Controller (MAC) to provide medium access to Gigabit Ethernet [Ins02]. Each of its two ports are connected to a Physical Layer Chip (PHYter) (DP83861 [Nat01]) via a standard GMII (Gigabit Media Independent Interface) bus. The PHYters enable Gigabit Ethernet over Copper line side access via standard Magnetics and normal RJ-45 connectors. The System side is connected via a POS-PHY Layer $3\left(\mathrm{PL}^{6}\right)$ [ATM00, PMC00] bus system to the Ethernet-CPLD (Complex Programmable Logic Device), a Delta39K100 CPLD [Cyp01].

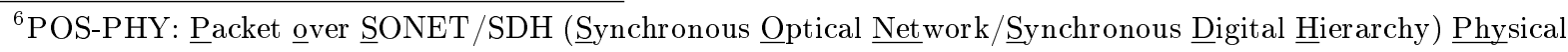
Layer
} 
In contrast to most other Ethernet MAC controllers produced for the PC market, the PM3386 is not using the PCI (Peripheral Component Interconnect) bus for system side connectivity, but it is equipped with a POS-PHY Layer 3 bus. This bus is used for point to point data framing in telecommunication equipment and has the advantage of not being a rather complicated memory mapped back plane bus like to PCI. The PL3 bus has 32 data bits and a sideband control channel for RX and TX traffic. Both can be operated at a maximum rate of $104 \mathrm{MHz}$. In addition, a 16 bit

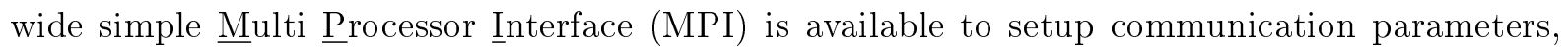
monitor traffic statistics, detect errors, or just reset different functional parts of the MAC. For example, this interface provides access to the number of frames received and the number of frames received with transmission errors. All details can be found in [PMC01].

Gigabit Ethernet enables communication with $1 \mathrm{GBit} / \mathrm{s}$ signaling rate. In real life, even raw data rates are lower, since data streams and data messages are encapsulated into Ethernet frames. This adds the overhead of header and trailer information (see [IEE00] for details). In addition, frames are separated by an Inter Packet $\underline{\text { Gap }}$ (IPG). Higher layer protocols reduce the achievable date rates further for very similar reasons. A practical example can be found in Section 3.4.

\subsubsection{Digital Signal Processor}

Running a custom application and the processing of data and network protocols, is performed in the GE-MN by a TigerSHARC [Ana02, Ana04] (Super Harvard Architecture) DSP from Analog Devices. It is a floating point 32 bit DSP with two execution units and $6 \mathrm{Mbit}$ embedded

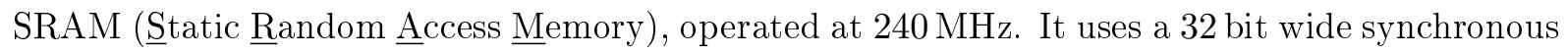
pipelined multi master TigerSHARC Cluster Bus (maximum frequency $100 \mathrm{MHz}$ ). In addition, the DSP features also four Link Ports. These point to point bus systems can run at a maximum frequency of $125 \mathrm{MHz}$ and carry data on eight data lines in a source synchronous Double Data Rate (DDR) scheme. Three sideband control lines are used and data transfer is always done in multiples of 128 Bytes (a Quad Word of this architecture).

Programing of this DSP is possible with the VisualDSP [Vis04] Software from Analog Devices, which provides an assembler, a $\mathrm{C} / \mathrm{C}++$ compiler, a debugger and an emulator integrated into an IDE (Integrated Development Environment). The DSP is booted by writing the binary program code via the VMEbus into its main memory and triggering an internal vector IRQ (Interrupt Request) to start program execution.

\subsection{Network Communication with the GE-MN}

Network communication with the GE-MN is possible via two standard Gigabit Ethernet ports. On these ports a number of protocols of different complexity and purpose are supported. The supported protocol of the lowest network layer is the Ethernet Protocol [IEE00]. It is directly processed by the MAC, which is (in case of ingress traffic) checking the Ethernet Frames for their validity and the correctness of the frame's Frame Check Sequence (FCS), and forwards

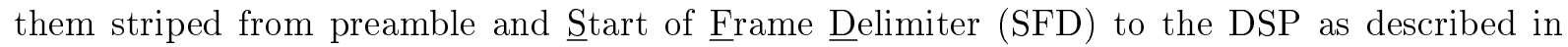
[PMC01, Rec00, Ins02]. The next higher layer protocols supported are the Address Resolution Protocol (ARP) [STD82], followed by a subset of the Internet Protocol (IP) [STD81a], including

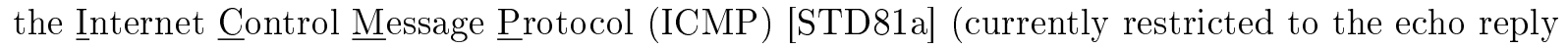
service). These protocols where originally defined in the Request For Comments (RFC) documents (compare [RFC87]) and are now collected as Internet Standards in the STD documents 
[RFC92, STD06, STD04, STD89, STD81a, STD80, STD81b, STD82, STD84, STD88a, STD88b]. The interaction of the different protocols is visualized in the network stack shown in Figure 3.3.

\subsubsection{Address Resolution Protocol - ARP}

The $\underline{\text { Address }}$ Resolution Protocol (ARP) [STD82] provides an automatic method of associating a 48 bit Ethernet Address used in the transmission in an Ethernet based Local Area Network (LAN) with a 32 bit IP address. A station using this protocol works by sending a Ethernet broadcast packet into the LAN querying for the Ethernet address of the IP address to which IP traffic should be sent and answering to similar requests for its own IP address with a ARP reply packet, sending back its Ethernet address. The so called ARP table is used to store this information.

\subsubsection{Internet Protocol - IP/ICMP}

The Internet Protocol (IP) [STD81a] provides two basic services in a packet based network: addressing and fragmentation. Addressing is the ability to identify stations and route packets to the intended receiving station. Fragmentation is the ability to send IP datagrams of a size bigger than the Maximum Transfer Unit (MTU) of the underlying network layer in several packets and to defragment and to reconstruct the full IP Datagram by collecting and reassembling the packets. For example, the maximum size of a IP Datagram is 65536 Octets $^{7}$, while the maximum payload ${ }^{8}$ of an Ethernet packet is 1500 Octets. Standard addressing and fragmentation is supported by the GE-MN.

The ICMP (Internet Control Message Protocol) protocol is an internal and required subprotocol of the IP protocol [STD81a]. It uses messages similar to IP Datagrams to back propagate error and status messages to other hosts. In addition, ICMP defines the echo reply service, better known by the utility that performs it as "ping". The echo reply service works by receiving ICMP echo request messages, exchanging source and destination address and sending the message with the same data back. This scheme enables several basic network tests. It can be tested if a host is reachable and up and running by checking if it is answering at all. The time between sending the request packet and the arrival of the answer can be measured and gives the real full round trip latency (or Round Trip Time, rtt). This latency measurement is in a small local area network dominated by the time the receiver needs to answer the echo reply request. In addition, this tests can be automatically repeated to get averages for the round trip latency and to measure the average packet error rate in a real environment. These tests are supported by the GE-MN and the results are presented in Section 3.4.

\subsubsection{User Datagram Protocol - UDP}

As the next next higher network layer above the IP protocol layer, the User Datagram Protocol (UDP) [STD80] is implemented in the GE-MN. UDP provides a connectionless and insecure ${ }^{9}$ application multiplexing messaging scheme. This means in practice that data is sent as messages (blocks of data) to a given port (application) of a host (IP address), possibly broken down into several smaller packets for transport in lower level protocols (fragmentation). IP/UDP

\footnotetext{
${ }^{7} 1$ Octet $=8$ bits

${ }^{8}$ payload: The part of the packet not belonging the protocol.

${ }^{9}$ in the sense of best effort data security
} 
communication does not provide any method of error correction or automatic retransmit on error, but (because of this) it is the protocol of the TCP/IP protocol family that provides the lowest latency. It is suited for real-time applications where a retransmit scheme breaks the realtime requirements. It also has the advantage over a connection oriented protocol (i.e. TCP, see Section 3.2.4) that applications are not bound to a specific connection stream, which could break or hang after a network/host/application failure and would need a complicated and time consuming reconnect scheme. As a further advantage it can provide a natural separation of data into logically related units (i.e. one Datagram per physical event) without the need for further structures, i.e. the Mark and Space separators needed in a stream.

\subsubsection{Transmission Control Protocol - TCP}

The connection oriented, reliable and data streaming Transmission Control Protocol (TCP) [STD81b] is not implemented in the GE-MN for several reasons. In addition to those mentioned in the discussion of the UDP advantages, the DSP in the GE-MN has limited resources not quite adequate to implement a complicated protocol like TCP (pages of specification: TCP $=180$ vs. UDP $=3$ ). For example the memory requirements for transmission buffers are comparatively large, since the TCP error correction requires to store all transmitted TCP segments until they are acknowledged to be received without error. As the egress link speed is 2 GBit, the limited memory resources in the GE-MN could easily be exceeded. Considering a further practical detail, the physics experiment of interest for this work produces data belonging together in events, which can be conveniently put into individual UDP datagrams. The data streaming nature of TCP would in fact introduce additional overhead.

\subsubsection{GE-MN Network Communication Model}

The intended default network communication mode with the GE-MN is to use UDP on top of IP and Ethernet to send and receive messages as shown in Figure 3.3. The application layer protocols on top of UDP are custom build to transport physics event data to and from the GEMN (described in Section 3.3) and to monitor and debug the status of the analysis applications running in the GE-MN. Obviously all supported protocols can be used at the same time. No error detection schemes beyond those in the standard protocols are implemented. This means that the most advanced error detection system available in the GE-MN is the 32 Bit $\underline{\text { Cyclic Redundancy }}$ Check (CRC) in the FCS of the Ethernet protocol. Frames without a valid FCS are dropped by the MAC. In the higher layers no transmission errors have been seen in practice, apart from corrupted packets due to obvious programming errors.

The required configuration parameters, which have to be set up in the GE-MN for a successful UDP/IP communication via Ethernet are the hardware address of the Ethernet interface (MAC address) and the IP network configuration. The latter consists of the IP address, network mask and default gateway. These parameters can be accessed as registers via the VMEbus, and are typically being set by the boot procedure. 
If a PC is used to communicate with the GE-MN, the standard facilities of its operating system can be used to allocate Datagram sockets $\left(\mathrm{BSD}^{10}\right.$ network programming model) and transmit and receive data, provided that protocols are used which are supported by the GE-MN (i.e. UDP/IP Datagram sockets). This simplifies and standardizes communication with the GE-MN, and enables it to communicate with basically every TCP/IP protocol family supporting system in existence. Debugging of the communication with the GE-MN is simplified by the existence of a large number of standard network diagnostic tools, such as "ping" or "wireshark" [Com07].

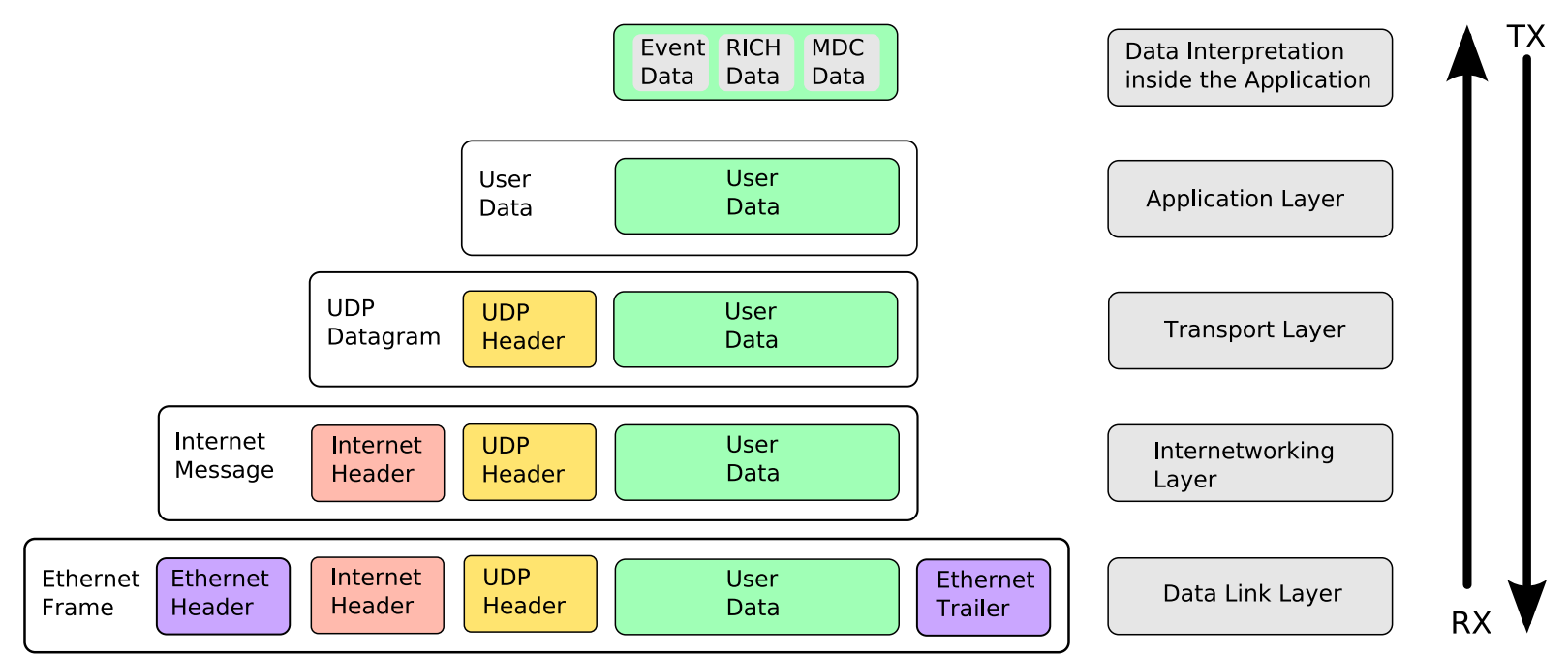

Figure 3.3.: GE-MN Network Communication Model (UDP/IP Network Stack).

The figure shows how data units are encapsulated in different protocol layers and passed through the IP/UDP network stack of the GE-MN. The different layers (described on the right side) add (TX) or remove (RX) their headers when passing data to the next or previous layer. The complete data packet per layer is on each layer shown in the correspondingly labeled frame.

${ }^{10}$ BSD: Berkeley $\underline{\text { Software }}$ Distribution 


\subsubsection{Multi-Node Network Topologies with GE-MN Systems}

The two network ports of the GE-MN enable the use of two basic network topologies: star topology or ring topology. The typical advantages and applications of these topologies are discussed in the next two sections.

\subsubsection{Star Topology}

Figure 3.4 (a) shows how to build star topology networks with several GE-MN systems. The advantage of this topology is its parallel architecture. Every system can communicate with every system directly, and the latency of this communication is constantly low, at least as long as the central switch is not reaching saturation and enough bandwidth is still available. In addition, data can be distributed to all systems directly via broadcasts, or by multi-casts if only a subset needs to be addressed.

The scalability of this topology is limited, because the number of ports in the switch as well as the achievable bandwidth in the switch are both limited. The workaround by cascading switches decreases the average available bandwidth per node and increases the average latency. Moreover, the resulting structure is no longer a real star, but it becomes a tree. In this case also the number of active network components starts to grow.

\subsubsection{Ring Topology}

Figure 3.4 (b) shows how to build ring topology networks with several GE-MN systems. This topology has two special locations: At one location data is injected into the ring, at the other location data is removed from the ring (they can be identical). Devices in the ring can only communicate directly with their two immediate neighbors. All other access are only possible via a number of hops via other nodes. This can result in a high latency, depending on the actual addressed device and the number of devices in the ring. On the other hand the bandwidth is independent of the number of nodes in the ring and also the number of active network components does not grow with the ring size. Two basic schemata are conceivable for parallel processing.

The fist schema assigns different sequential task of the analysis to sequential nodes, which then form a pipeline. For example, the first data packet is injected into the ring and travels to node 1. This node executes step A of the analysis and sends the result to node 2 . In node two part $\mathrm{B}$ of the algorithm is executed and the result is sent to node 3 . While node 2 is processing part $\mathrm{B}$, node 1 is executing part $\mathrm{A}$ of the algorithm for packet number two. This pipeline speeds up data processing by processing different parts of the analysis in parallel. The number of nodes on the ring is tightly coupled to the partitioning of the algorithm, which limits the scalability.

In the second schema data is injected in packets to be analyzed into the ring. These packets circulate the ring until they pass an idle node, which removes them form the ring and analyzes them. The results are again injected into the ring to be extracted at the extraction location, where the results are for example sent to a server for storage. The advantage of this schema is its scalability. If this system has to be extended it is only necessary to add more nodes to the ring. Another advantage is the automatic buffer management realized by the fact that packets circulate in the ring until they find an idle node. A disadvantage of this scheme is the not really deterministic latency, especially at a high saturation when it can happen that packets travel an essentially random number of times around the ring before they are processed. 


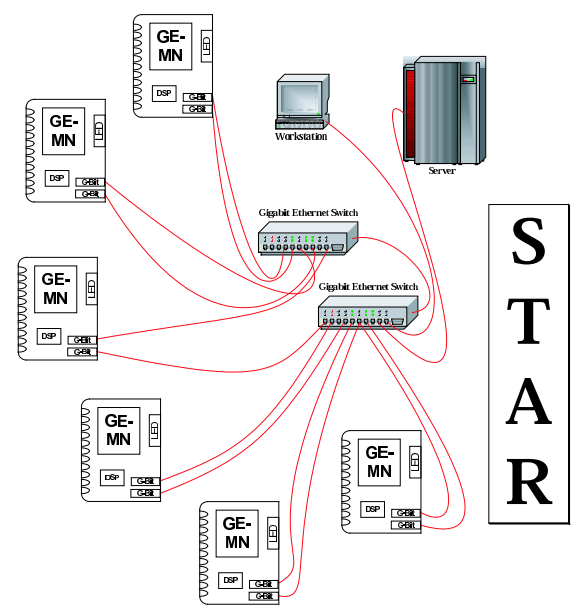

(a) Star Topology

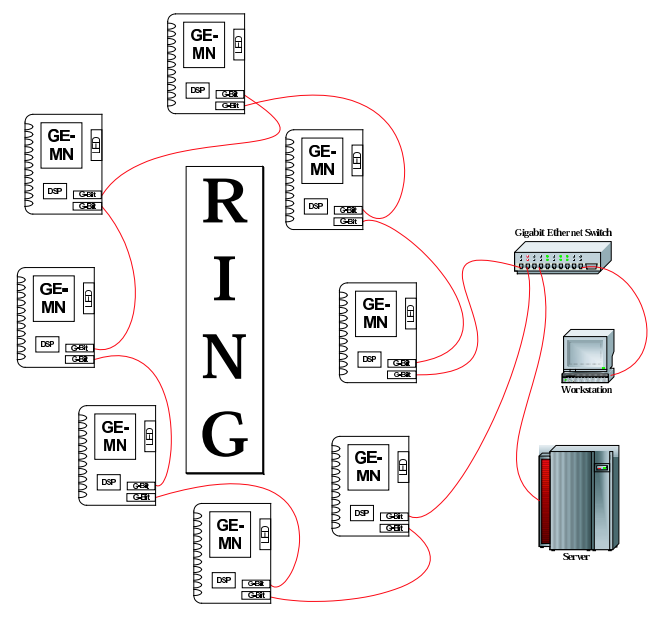

(b) Ring Topology

Figure 3.4.: Network Topology: Ring and Star

The figure in (a) shows a star topology and the figure in (b) shows a ring topology, both built up with GE-MN systems. The ring, in this case, is actually a double ring, because the Gigabit Ethernet links of the GE-MN are full duplex links.

\subsection{GE-MN - Software}

Design, development and implementation of the GE-MN hardware as such did not directly produce an usable system. Additional software is needed to make the hardware execute useful tasks. The hardware programming (VHDL) of the CPLD chips enables all the communication paths between the different components as described in Section 3.1.1. The next software layer is the programming framework for the DSP which provides the facilities needed for applications running in the DSP to receive and send network packets using the protocols described in Section 3.2. This framework implements a central Finite $\underline{\text { State }}$ Machine (FSM) running basic services and applications.

The data packets from the network are transmitted and received via DMA transfers from the TigerSHARC Link-Ports. Handling of DMA access is performed in interrupt service routines, which setup empty buffers to receive data, flag buffers that have been successfully received and transmit buffers which are scheduled to be send out by services or applications. The buffer management implemented in the GE-MN is illustrated in Figure 3.5. 


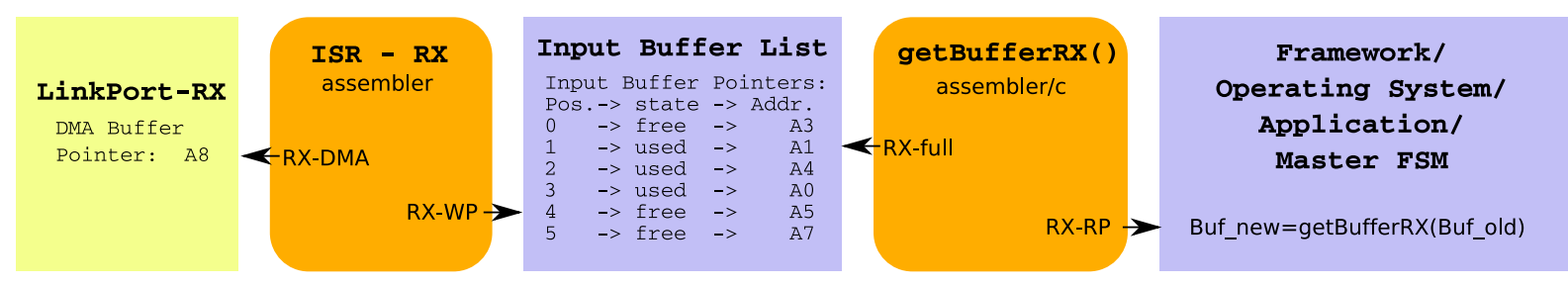

\section{DATA FLOW}

Hardware Module

Interrupt Safe

Framework Layer

Figure 3.5.: Receive Network Buffer Management in the GE-MN.

Buffer management in the GE-MN is, with the exception of packets for defragmentation, implemented as an exchange of buffers. This figure illustrates this for the receive (RX) data flow from the network. Ethernet frames are received by the DSP via its receive Link-Port. The associated DMA controller transfers the data directly to a place in the memory of the DSP, which is starting in the example at the location pointed to by the pointer A8. After completing the transfer the Interrupt Service Routine for RX traffic (ISR-RX) is triggered. This exchanges the buffer pointers indicated by RX-DMA and RX-WP, increments RX-WP and sets up a new DMA transfer. This exchange moved the pointer to the just received data into the input buffer list, which is implementing a ring buffer. Data buffers are withdrawn from the ring buffer when the getBufferRX function is called by exchanging the work buffer of the framework with the buffer indicated by RX-full, which is also incremented to indicate that the exchanged pointer can be reused. The functions in the red boxes operate atomically, and thus interrupt safe, by halting interrupt processing during their execution. The transmit buffer management follows the same scheme (reverse previous description). The buffer exchange scheme for buffer management is efficient because no unnecessary movement of data in memory is performed in the whole process, only pointers are exchanged between different arrays.

The implementation of the processing of the different network protocols is done as stacked services in analogy to the four layers of the TCP/IP network stack model as shown in Figure 3.3 and described in Section 3.2. After an incoming Ethernet frame has been transferred to a buffer, it is inspected and interpreted in several steps. Default behavior in all steps is to drop the data in the buffer (and reuse it as free), if an unsupported protocol or corrupted data is found.

The first step is to determine if the Ethernet frame carries ARP or IP traffic. ARP packets are processed immediately by the ARP service as described in Section 3.2, while IP datagrams are processed as step two by the IP service, which checks for and handles ICMP packets, manages defragmentation and hands off correctly addressed, error free and defragmented or not fragmented packets to the next network layer. The following UDP layer is multiplexing UDP packets according to their port number to the appropriate application.

In this framework, services and applications are implemented as functions in the programming language $\mathrm{C} / \mathrm{C}++$, which are compiled all together when building the firmware (compare Section 3.1.4). In other words, there is no enforced separation of an application from the framework and no memory protection is enforced. Cooperative multitasking has to be explicitly built into the application. 
As mentioned, an application is implemented as a function (or set of functions), being called by the central FSM of the framework. As shown in Figure 3.5, getBufferRX can be used to get the Ethernet frame buffers, and in fact this is done by the framework to get the buffers to process from the network stack. The interpreted buffers are then pushed to the appropriate application. The programming model inside the GE-MN framework is not based on the BSD socket model, which is used on all modern operating systems. Instead, whole defragmented datagrams are pushed to the application, which has to interpret them appropriately. Zero additional overhead interpretation of a buffer is performed by casting ${ }^{11}$ the pointer to its base address into a pointer to a structure of the appropriate type. This is possible because of the fixed positions of the data fields relative to the base address of a buffer. This cast operation is used for buffer processing in the framework as well as in the application to interpret the data appropriately.

\subsection{Network Performance}

The generic term network performance of a system can be split into two conceptually different parameters: latency and bandwidth (throughput). Bandwidth is the amount of data that can be transported via the interconnect in a certain time. Latency is given here as the full round trip delay time. This is the time the sender of a message has to wait for an answer. Bandwidth and latency are not completely independent, since a faster link needs less time to transfer a data packet and thus has a lower possible latency. In local area networks this pure transfer time is usually not the main contribution to the latency, but processing times for check sum checking, buffer management, in system data transport and processing of higher layer protocols dominate the latency. The bandwidth becomes strongly latency related if the sender of data has to wait for acknowledges of successful transmissions.

\subsubsection{Network Throughput}

The network throughput of the GE-MN has been measured by using a Personal Computer (PC) and several simple test programs. The program "udp_tx" is used to send UDP messages to the GE-MN containing the number of the message and some additional arbitrary payload. The program provides an easy facility to change the number of messages transmitted per second. A test application in the GE-MN is used to receive the messages and to inspect them. The transmitted message number enables the program to detect dropped packets and packets out of order. All problems detected by the GE-MN are reported by sending a small status message to a monitor application running on a second PC. The data messages are further processed by incrementing the message number and sending them back to the originating PC, where similar checks as in the GE-MN are performed to detect problems. The full round trip bandwidth using IP/UDP messages of the GE-MN has been measured by this method to be $\approx 20 \mathrm{MB} / \mathrm{s}(20 \mathrm{MB} / \mathrm{s}$ in transmit and at the same time $20 \mathrm{MB} / \mathrm{s}$ in receive direction). It is mainly limited by the actual implementation of the Link Port to POS-PHY Layer 3 bus interface. This interface is challenging, not by concept, but by the fact that the TigerSHARC Link Ports are source synchronous ${ }^{12}$ DDR $^{13}$ point to point links. The exact implementation details are: clock rate TigerSHARC Link Ports $30 \mathrm{MHz}$ DDR $\rightarrow$ transfer rate limit: 8 Bit width at $30 \mathrm{MHz}$ DDR $\rightarrow 60 \mathrm{MB} / \mathrm{s}$. The TigerSHARC

\footnotetext{
${ }^{11}$ cast: type conversion/interpretation in $\mathrm{C} / \mathrm{C}++$

${ }^{12}$ The transmitter outputs the clock and data synchronously

${ }^{13}$ DDR: Double Data Rate; data is valid on on rising and falling edge of the clock
} 
Link Port protocol overhead is $37,5 \% \rightarrow 37,5 \mathrm{MB} / \mathrm{s}$ maximum raw data rate. The complicated part in implementing the Link Ports interface is to generate a internal clock signal inside the CPLD that allows stable transmission conditions on these links. It is accomplished by deriving the clock from the same system clock used by the DSP for its own clock generation, using the Phase Locked Loops (PLL) clock multiplier/divider and phase shifter of the CPLD. The "obvious" way of using the Link Port clocks as direct clock inputs is not possible, because the Link Port clock signals also carry in-band transmission information (protocol, token request,...), and this not always switching clock would not allow a stable operation of the PLL inside the CPLD. An additional detail of the Delta39K architecture is the absence of a signal path from logic nets to the dedicated clock nets, which prevents other "tricks" from working. The maximum possible gain by increasing the Link Port clock rate is a factor of four, but since this "clock tuning" is very time consuming and the network performance is already sufficient for the current applications (compare Chapter 4), there was no immediate need for further optimization.

\subsubsection{Network Latency}

The network latency of the GE-MN has been measured using a standard Linux PC and the standard ICMP echo reply method. This method is better known as "ping" by the name of the standard tool performing it. In Figure 3.7 the results from different ping tests are summarized. It should be noted that if one calculates the data throughput from this numbers the result will be quite low. For example in the second ping test: $1000000 * 1000$ Bytes $/(219687 \mathrm{~ms})=4.34 \mathrm{MB} / \mathrm{s}$. This is due to the fact that (in these tests) ICMP echo reply messages are not sent out before the reply for the previous message arrived. This is equivalent to only one packet being in transmit at a given time.

The probability of a packet loss in a realistic LAN environment can also be calculated to be equal to $2,77 * 10^{-6}$ from Figure 3.7. In comparison to the network performance of a fast standard

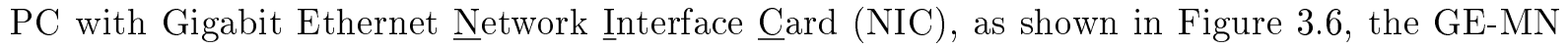
has not only the advantage of lower average round trip latency, but also that the deviations in the round trip latency are much smaller, leading to advantages in hard real-time applications.

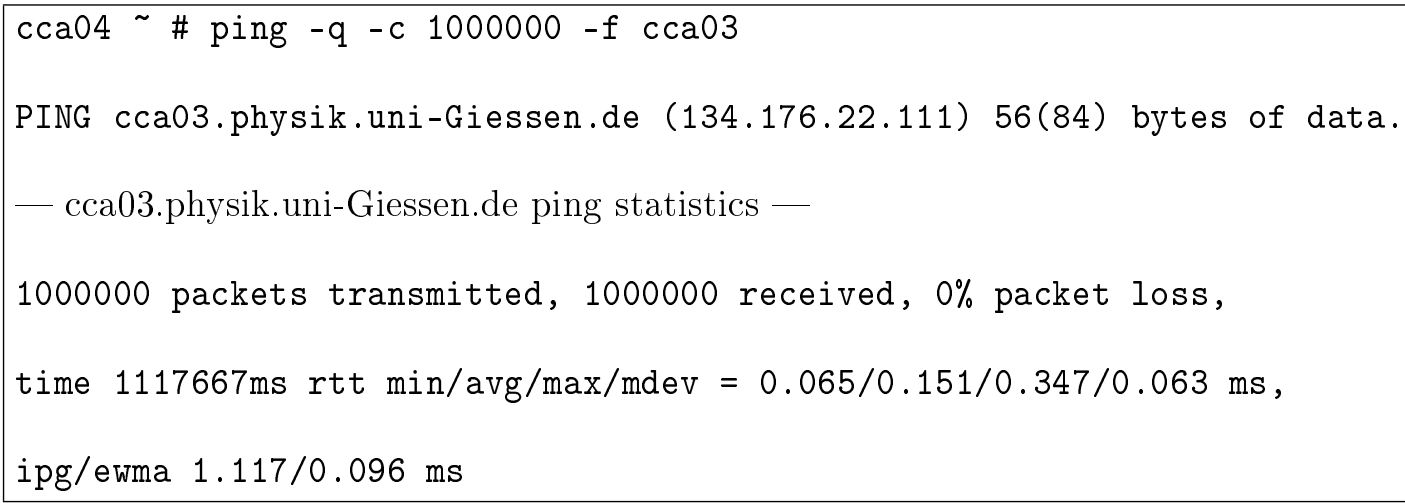

Figure 3.6.: Typical PC Network Latency (Gigabit Ethernet).

Packet Size: 64 Byte $\rightarrow$ Packet Loss: 0\%; Average Round Trip Latency: (151 \pm 63$) \mu$ s, Packets checked: 1000000 


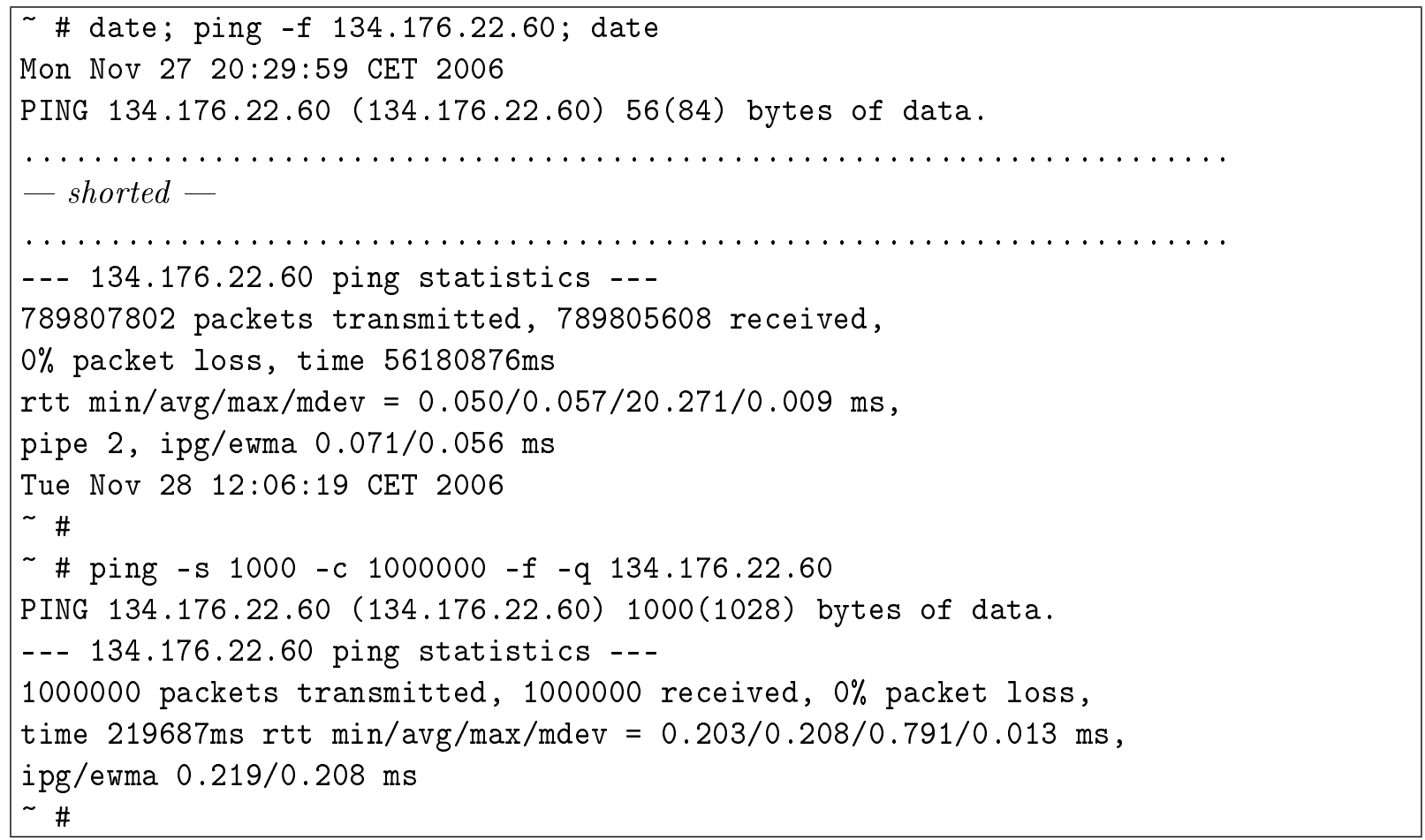

Figure 3.7.: Example "ping" Measurements of the GE-MN.

Example results obtained using the standard network analysis tool "ping". The IP address of the tested GE-MN is 134.176.22.60 Netmask 255.255.255.128, which means it is tested in the normal network of the university.

a) Packet Size: 64 Byte $\rightarrow$ Packet Loss: $2,77 \cdot 10^{-4} \%$;

Average Round Trip Latency: $(57 \pm 9) \mu$ s measured over a time of $18,5 \mathrm{~h}$

b) Packet Size: 1000 Byte $\rightarrow$ Packet Loss: $0 \%$;

Average Round Trip Latency: (208 \pm 13$) \mu \mathrm{s}$, Packets checked: 1000000

No duplicates or damaged packets have been observed in the application layer. 


\subsection{Bibliography}

[Ana02] Analog Devices, Inc; One technology Way, Norwood, Mass. 02062-9106; TigerSHARC DSP Microcomputer ADSP-TS101S Preliminary Datasheet; Rev.PrE edition (February 2002); URL http://www.analog.com

[Ana04] Analog Devices, Inc; One technology Way, Norwood, Mass. 02062-9106; ADSP-TS101 TigerSHARC Processor Hardware Reference; revision 1.1 edition (May 2004); Part Number 82-001996-01; URL http://www.analog.com

[ATM00] ATM; The ATM Forum, Worldwide Headquarters 2570 West El Camino Real, Suite 304, Mountain View, CA 94040-1313; The ATM Forum Technical Committee Framebased ATM Interface (Level 3) (March 2000); URL http://www.atm.org

[Cad06] Cadence Design Systems; NC-Sim (2006); URL http://www.cadence.com

[Com07] Gerald Combs et al.; Wireshark Website (February 2007); URL http://www. wireshark.org

[Cyp01] Cypress Semiconductor Corporation; 3901 North First Street, San Jose, CA 95134; Delta39K ISR CPLD Family; rev. c edition (December 2001); URL http://www. cypress.com

[Cyp02] Cypress Semiconductor; 9901 North First Street, San jose, CA 95134, (408) 943-2600; Warp HDL Development System Users Guide (May 2002); URL http: //www . cypress . com

[Her02] Richard Herveille; Wade D. Peterson et al.; Specification for the: WISHBONE System-on-Chip (SoC) Interconnection Architecture for Portable IP Cores (OpenCores Organization, 2002); revision: b.3 edition; URL http://www.opencores.org/ projects.cgi/web/wishbone/wishbone

[IEE00] IEEE Computer SocIETy; 802.3; IEEE Standards (The Institue of Electrical and Electronics Engineers, Inc, 3 Park Avenue, New York, NY 10016-5997, USA, 2000); revision 2000 edition; URL http://www. ieee802.org

[Ins02] The Institute of Electrical and Electronics Engineers, Inc; 3 Park Avenue, New York, NY 10016-5997, USA; IEEE Std 802.3; 2002 edition (August 2002); URL http://www. ieee.org

[Nat01] National Semiconductor Corporation; DP83861VQM-3 EN Gig PHYTER 10/100/1000 Ethernet Physikal Layer; 1st edition (April 2001); URL http://www. national.com

[Pet93] Wade D. Peterson; The VMEBus Handbook (Scottsdale - Zaltbommel, 1993); 3rd edition

[PMC00] PMC-Sierra, Inc; 105-8555 Baxter Place Burnaby, BC, Canada V5A 4V7; POS-PHYLevel 3 Saturn Compatible Packet over SONET Interface Specification for Physical und Link Layer Devices; pmc-1980495, issue 4 edition (June 2000); URL http://www . pmc-sierra.com 
[PMC01] PMC-Sierra, Inc.; 105-8555 Baxter Place Burnaby, BC, Canada V5A 4V7; PM3386 S/UNI-2xGE Dual Gigabit Ethernet Controller Datasheet; issue 7 edition (July 2001); URL http://www.pmc-sierra.com

[Rec00] JöRG RECH; Jetzt klapts auch mit Kupfer; Heise Verlag; c't ausgabe 14 edition (July 2000)

[RFC87] RFC 1011 - Official Internet Protocols (May 1987); URL http://rfc.net/rfc1011. $\mathrm{html}$

[RFC92] RFC 1311 - Introduction to the STD Notes (March 1992); URL http://rfc.net/ rfc1311.html

[STD80] STD 6 - User Datagram Protocol (August 1980); URL http://rfc.net/std6.html

[STD81a] STD 5 - Internet Protocol (September 1981); URL http://rfc.net/std5.html

[STD81b] STD 7 - Transmission Control Protocol (September 1981); URL http://rfc.net/ std7.html

[STD82] STD 37 - An Ethernet Address Resolution Protocol - or - Converting Network Protocol Addresses to 48.bit Ethernet Address for Transmission on Ethhernet Hardware (November 1982); URL http://rfc.net/std37.html

[STD84] STD 41 - A Standard for the Transmission of IP Datagrams over Ethernet Networks (April 1984); URL http://rfc.net/std41.html

[STD88a] STD 42 - A Standard for the Transmission of IP Datagrams over Experimental Ethernet Networks (February 1988); URL http://rfc.net/std42.html

[STD88b] STD 43 - A Standard for Transmission of IP Datagrams over IEEE 802 Networks (February 1988); URL http://rfc.net/std43.html

[STD89] STD 3 - Requirements for Internet Hosts - Communication Layers (October 1989); URL http://rfc.net/std3.html

[STD04] STD 1 - Internet Official Protocol Standards (July 2004); URL http://rfc.net/std1. html

[STD06] STD Index (April 2006); URL http://rfc.net/std-index.html

[Syn06] Synplicity, Inc.; Synplify Pro (2006); URL http://www.synplicity.com/

[Vis04] Compiler and Library Manual for TigerSHARC Processors; One technology Way, Norwood, Mass. 02062-9106; revision 1.1 edition (March 2004); Part Number: 82-00033603; URL http://www . analog.com

[VIT06] VITA; VMEbus International Trade Association (2006); URL http://www.vita.org

[Wis06] Wishbone People; Wishbone Website (2006); URL http://www.opencores.org/ projects.cgi/web/wishbone/wishbone 


\section{4. $\mathrm{RICH}$ to $\mathrm{MDC}$ Correlation}

\section{Contents}

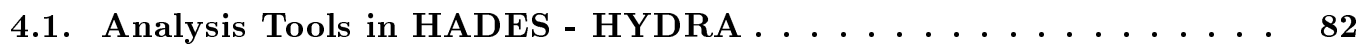

4.1.1. Analysis Chain and used Data Set . . . . . . . . . . . . . 82

4.1.2. Definition of good Leptons $\ldots \ldots \ldots \ldots$. . . . . . . . 83

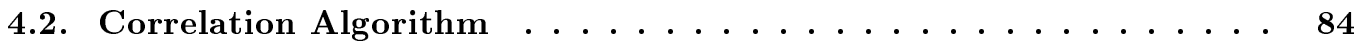

4.2.1. Correlation Basics . . . . . . . . . . . . . . . . . 84

4.3. Implementation Details . . . . . . . . . . . . . . . 85

4.3.1. Calculating the Crossed Cells - calcXed ()$\ldots \ldots \ldots$. . . . . 86

4.3.2. Parameter Implementation in the GE-MN . . . . . . . . . . . . . . 90

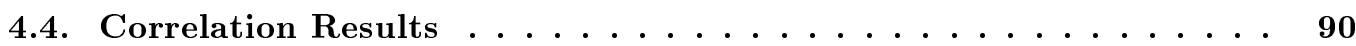

4.4.1. RICH IPU Performance . . . . . . . . . . . . . . . . . . . . . . . . . 91

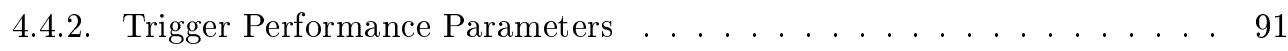

4.4.3. Online Analysis Considerations . . . . . . . . . . . . . . . 92

4.4.4. Offline Analysis Considerations . . . . . . . . . . . . . . . 93

4.4.5. Correlation Analysis $-\mathrm{p}+\mathrm{p}$ at $2,2 \mathrm{GeV} \ldots \ldots \ldots \ldots 5$

4.4.6. Correlation Analysis $-\mathrm{C}+\mathrm{C}$ at $1,0 \mathrm{AGeV} \ldots \ldots \ldots \ldots$

4.4.7. Correlation Analysis $-\mathrm{Ar}+\mathrm{KCl}$ at $1,756 \mathrm{AGeV} \ldots \ldots \ldots 117$

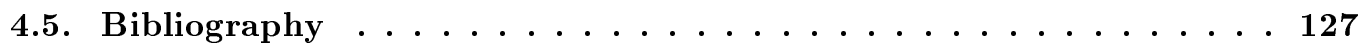

The online HADES RICH ring information, as provided to the trigger system by the RICHIPU (compare Section 2.3 and Figure 2.6), is largely fake dominated. The correlation of the RICH ring information with the signals from other detectors could identify a large fraction of the fakes, and thus significantly reduce the number of fake RICH rings which presently have to be taken into account in further processing. In addition, only few events actually contain the real leptons, which produced real rings in the RICH. The detector data samples which provide the most detailed information to reconstruct the tracks of charged particles are the data samples taken by the tracking drift chambers (MDC). These have the best spacial resolution available in HADES.

The current implementation of the HADES trigger does not use any information provided by the MDC drift chambers (compare Section 2.4 and Section 2.12) and there is no data path for the MDC data to reach the trigger system. Both issues are being addressed by the upgrade of the HADES DAQ discussed in Chapter 5.

The correlation of the RICH ring data with the MDC wire data provides one possibility to reject RICH fakes and enrich the recorded data samples with events containing real leptons. One method to perform this correlation is described in detail in this chapter. Further, the detailed results obtained by applying the correlation method to data sets from different beamtimes with different reactions are presented. In addition, a short overview of the analysis framework used in 
HADES will be given, since it is essential to extract high quality results from the experimental data. This chapter will conclude with the discussion of the performance of the correlation method running in the GE-MN and processing real data (Chapter 3).

\subsection{Analysis Tools in HADES - HYDRA}

HADES uses a simulation and data analysis package called HYDRA (ㅂADES System for Data Reduction and Analysis) [SG03a, HADa]. This framework provides HADES specific methods to retrieve raw data from the files of the DAQ (Data Acquisition System), store the raw data samples in an object oriented way and run standardized analysis methods on the data. Results are stored and propagated through the whole offline analysis chain in persistent objects in "*.root" format files. HYDRA is heavily based on the ROOT framework [Bru97].

ROOT is a $\mathrm{C}++$ interpreter based software framework in common use in $\underline{H} i g h$ Energy Physics (HEP). It provides methods and basic data structures necessary for the convenient analysis of data. The features range from basic functionality like data IO frameworks and platform independent data storage to more advanced features like generating histograms or apply fitting procedures to data, collection classes, 2D/3D graphics, uㅡ Time Type Identification (RTTI) and many more. All details are available in [Bru07].

\subsubsection{Analysis Chain and used Data Set}

The event builder collects the raw data samples from the individual detectors via the DAQ system, and stores them in "*.hld" files (ㅂADES List Data). These data samples are processed with

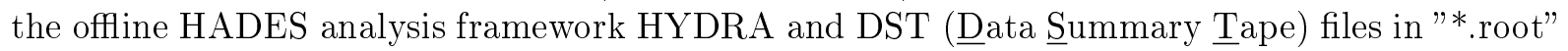
format are generated. This process is called DST production and it is repeated iteratively to produce different DST generations. This is necessary, because the detector calibration, detector alignment and other parameters are improved by the ongoing analysis. The DST files are then used in the physics analysis. They contain the relevant information like track data, PID (Particle Identification), particle momenta and particle energies. In addition, a lot of the raw detector response data samples are removed, because these are not used in the later analysis. For the analysis of a trigger algorithm these data sets are required, thus special DST files are generated for the analysis presented later, which still contain the raw detector information of the MDC detector and the RICH IPU.

The data sets stored in the DST files are not only the data sets recorded after a positive HADES second level trigger decision, but also the so called downscaled data. The downscaled data samples are stored independently of the second level trigger decision. The ratio of the (by the first level trigger positively triggered) events recorded independently of the second level trigger decision is called the down scaling factor. This is of special importance for the analysis of a trigger method operating on a higher level as the HADES first level trigger, since the downscaled data samples allow to investigate which effect a trigger algorithm has upon this data independent of higher layer triggers. This downscaled data samples are used to produce the analysis data sets which contain the data samples and analysis information required by the analysis presented in this chapter. 


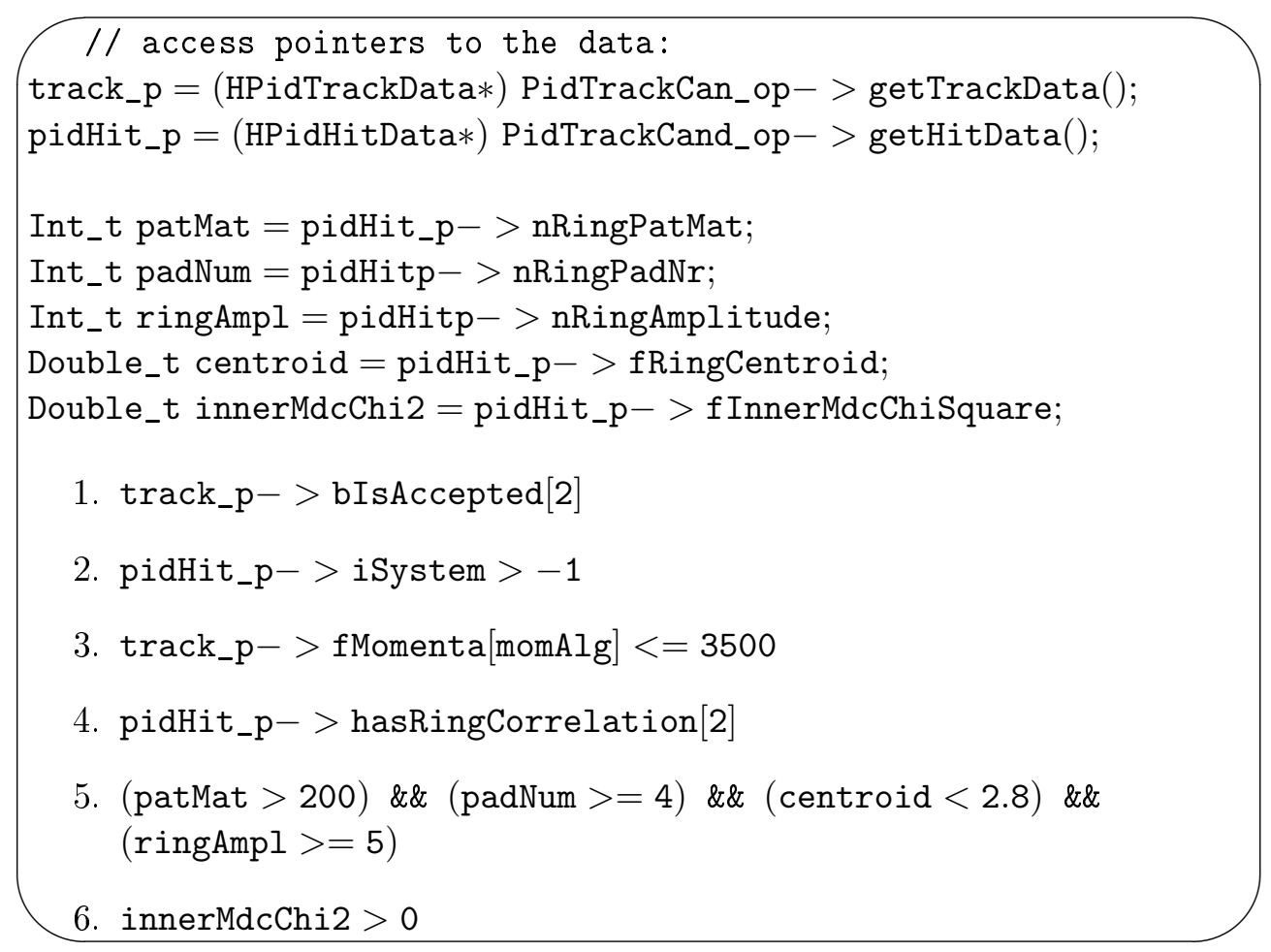

Figure 4.1.: Definition Good Leptons for the Real Data.

In this work a track candidate (HPidTrackCand) in the experimental data is considered a good lepton track (henceforth a good lepton) if it passes the set of cuts shown above in pseudo code. The numbered Boolean conditions have the following meaning: The track candidate has to be accepted by the spline tracking algorithm (1). A correlation with a TOF or Shower (META) hit is required (2) and a high momentum cutoff is used (3). The track has to be correlated with a ring in the RICH (4) which has to fulfill a quality criteria (5). The criterion that the track has to have a good $\left(\chi^{2}>0\right)$ value in the track fit of the inner MDC chambers (6) was not used, since it is strongly reducing the statistics. However it has been verified that the results (especially efficiency and enhancement) presented later are not significantly affected. Events that contain one or more good leptons are called good lepton events.

\subsubsection{Definition of good Leptons}

In the offline analysis of the correlation algorithm the information which event has a real (not fake) lepton track is used in several places, as for example in the calculation of efficiency and enhancement. For a consistent definition of a lepton the criteria in Figure 4.1 are used. These are based on analysis data available for all investigated experimental data. It has to be stressed that the results of these cuts is somewhat dependent on the actual DST generation used, as the alignment and the calibration enter the tracking procedure. The individual DST generation used is stated at each individual analysis. 


\subsection{Correlation Algorithm}

The correlation between two detectors is performed by comparing the hits in one detector with the hits in the other detector and rejecting events in which a hit is detected in one detector only. For the correlation of RICH with MDC this would mean to correlate rings from RICH (data: $\vartheta, \phi)$ with tracks from the inner MDC chambers (data: straight line in space). Since the RICH ring information is not defining a track by itself, it would be natural to start the correlation by investigating at a track from MDC, following this straight line (track) through the RICH and calculating the theoretical RICH $(\vartheta, \phi)$ hit. The comparison of the calculated hit with the real hit would provide a method to reject fake RICH rings and trigger on events with good leptons.

In practice this method cannot applied in this form, because pointing with a MDC track back to the RICH requires a reconstructed track. But these are available only after track reconstruction, which is a time consuming, highly calibration dependent and challenging task and is performed as part of the offline analysis (compare Section 2.4). Thus, these results are completely unavailable for the HADES online system. Without further processing and analysis the MDC chambers provide online only the raw TDC information, which is at the present time also inaccessible to the trigger system, albeit this will change in the near future due to the general upgrade of the HADES DAQ and trigger system described in Chapter 5.

\subsubsection{Correlation Basics}

A method to correlate RICH rings with MDC information, with the goal to reduce the fake RICH rings suitable for an online (real-time) system, can be implemented in a different way as the one described above. A real ring in the RICH detector is detected as the signal of a fast $\left(\gamma>\gamma_{t h r},\right)$ charged particle (i.e. for HADES an $e^{+}$or $e^{-}$) passing through the detector (see Section 2.3). Since this particle travels in a straight line before entering the magnetic field, it also traverses the inner MDC chambers in a straight line, producing a signal in the different drift cells in the different layers of the MDC detector. Assuming that a ring in the RICH detector is produced by a particle which came from the target's position, it is possible to assume a specific straight track for its path. This straight track is defined by the nominal target position as emission point and the direction is determined by the RICH detector's $(\vartheta, \phi)$-information, which itself is derived from the ring center by the RICH IPU. Following this straight track through the MDC chambers it is clear that the hit sensitive volumes (drift cells) should have seen a signal from this particle. This procedure follows the same basic idea for the track finding as the one described in Section 2.11. The Investigating of all RICH rings for corresponding hit MDC drift cells provides the trigger method discussed in the following sections. It selects good RICH rings, or better the RICH rings with a high probability to be generated by an electron or positron (a lepton candidate), and thus allows to enrich lepton events.

In practice a track derived from a RICH ring is not defined well enough to be expected to match exactly a certain calculated MDC cell. Instead a matching window (henceforth also called $W_{m}$ ) has to be used, allowing also nearby drift cells to be considered as matches, as long as they are inside the matching window. The reasons for the inaccuracies are mainly of geometrical nature. First, the $(\vartheta, \phi)$-information of the RICH rings available online is not much better defined as given by the granularity of the photo-detector detector plane of the RICH (compare Section 2.3 and Figure 2.4). Second, the target position is not a point in space, but the target is an extended object (compare Section 2.1), which leads in most cases to a difference of the real source point of the track to the nominal one used in the calculations. In addition, scattering in the detector 
material can change the direction of a track.

The required size of the correlation window can be determined by investigating the correlation between the drift cells which have fired, in terms of their wire number, and the calculated drift cells which should have fired. It is more meaningful for an online system to compare wire numbers instead of the polar angle and azimuthal angle RICH to MDC correlations, since the MDC angular information is tied to a track which is only available after track reconstruction. An example of a histogram showing the wire number correlation is shown in Figure 4.6.

A particle passing through a detector has a probability smaller than one to be detected. This also applies to a particle passing through the different layers of the MDC detector. Instead of requiring exactly the calculated number of corresponding fired drift cell for each MDC layer, the approach to count the number of layers with fired drift cells inside the matching windows (matched layers) is used. A minimal number of matched layers (henceforth also called $L_{m}$ ) is used as a threshold for the condition that the RICH ring is considered to have a good probability to have a corresponding real electron or real positron track. A natural and very conservative (safe) choice of this threshold is the minimum number of layers required by the offline cluster finder, which is at least four (as discussed in Section 2.11). This choice is very conservative, because a track (even if a real track and not a fake track) without this number of hit layers is rejected by the cluster finder, and thus not found and used in any analysis anyway.

\subsection{Implementation Details}

In order to implement the RICH to MDC correlation several technical aspects have to be handled. The Data Acquisition System (DAQ) is reading out the TDC channels (compare Section 2.4) by motherboard number (abbr.: $m b o$ ) and channel number (abbr.: $t d c$ ) for each sector (abbr.: sec) and module (abbr.: mod). The (by position ordered) drift cell number (abbr.: cel) defined per MDC layer (abbr.: lay), as described in Section 2.4, is not used online. Every drift cell has a corresponding attached TDC with a well defined channel number located on a certain motherboard, but the mapping is not systematic and not all defined TDC channel numbers have actually a TDC connected to a sense wire. These relation and the used naming convention can be found in Table 4.1 .

\begin{tabular}{|c|c|c||c|c|c|}
\hline Offline Name & Numbering & abbr. & abbr. & Numbering & Online Name \\
\hline \hline Sector & $0-5$ & $s e c$ & $s e c$ & $0-5$ & Sector \\
\hline Module & $0-1$ & mod & mod & $0-1$ & Module \\
\hline Layer & $0-5$ & lay & $m b o$ & $0-15$ & Motherboard \\
\hline Cell & $0-255$ & $c e l$ & $t d c$ & $0-95$ & TDC Channel \\
\hline
\end{tabular}

Table 4.1.: Mapping Table, Online to Offline.

The right hand side numbers used by the online system need to be converted to the offline notation on the left side for the further calculation. The practical implementation is a LUT

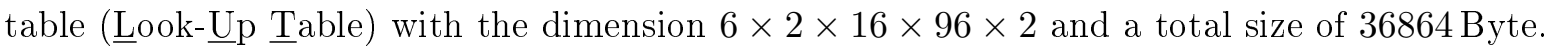

In addition, this mapping can change in time when parts of the detector are rewired, making it impossible to derive a static generalized mapping by calculation. Instead these features of

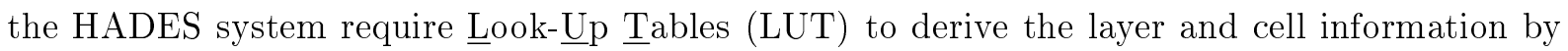


implementing the functions:

$$
\begin{aligned}
& \text { SMBT2LayCel_LUT }[\mathrm{sec}][\mathrm{mod}][\mathrm{mbo}][t d c][0]=l a y \\
& \text { SMBT2LayCel_LUT }[\mathrm{sec}][\mathrm{mod}][\mathrm{mbo}][t d c][1]=\mathrm{cel}
\end{aligned}
$$

This LUT has a size of $6 \cdot 2 \cdot 16 \cdot 96 \cdot 2$ Bytes $=36 \mathrm{~KB}$.

The SMBT2LayCel_LUT LUT (Equation (4.1), Equation (4.2)) is filled by retrieving the (lay; cell) data by querying the HADES online Oracle database. The last parameter selects between layer and cell information and enables the use of one common LUT. In addition to this the LUT channelsPerBoard[sec][mod] [mbo] is filled. It contains the number of TDC channels per motherboard (mbo) which is needed to limit loops over TDC channels to meaningful intervals.

The layer and cell (lay; cel) information is needed, because it is possible from very few geometrical base data to directly calculate the point where a straight line in space would cross a drift cell in each of the layers of a module in a sector of the MDC detector in terms of the (lay; cel) coordinate. This is the case, because the relation between actual position and layer and cell number is systematic and governed by geometry (compare Figure 2.7 and Section 2.4). The function used to calculate the crossed cell for a given MDC module and MDC layer for a $\mathrm{RICH}$ hit $\left(\vartheta_{\mathrm{RICH}}, \phi_{\mathrm{RICH}}\right)$, described in detail in Section 4.3.1, is:

$$
\operatorname{calcXed}[\bmod ][\text { lay }]\left[\vartheta_{\mathrm{RICH}}\right]\left[\phi_{\mathrm{RICH}}\right]=\mathrm{cel}
$$

The data needed to initialize the different look-up tables is obtained by querying the HADES Oracle [Ora06] database system, where all geometry and experimental run dependent information is stored. In principal the straightforward approach to implement a straightforward 2D LUT mapping the RICH $(\vartheta, \phi)$ information directly to the hit cell in each of the 12 MDC layers could be taken, but simulations did show that a LUT with sufficient precision is too large (several $\mathrm{MB}$ ) to be implemented in the limited memory resources of the GE-MN. This is even the case, if the granularity of this 2D LUT (determining precision and size) is lowered to a point where its resolution is completely insufficient.

A compact summary of the trigger method is: For each ring from the RICH IPU calculate for each MDC layer of the same sector the drift cell that should have been hit and check in that layer the neighboring cells for hits within the matching window. Then, the number of layers which could be matched in this way is counted. If for one of the rings the required minimum number of layers was reached or exceeded the event is accepted. The technical details of the implementation of the whole method are explained in the directly following sections, while Section 4.4 discusses the results of the method when applied to real data from several beamtimes.

\subsubsection{Calculating the Crossed Cells - calcXed ()}

The ideas for an efficient implementation of the function calcXed() are mainly based on geometrical considerations. The functions already existing inside the HADES framework implementing an almost identical functionality are based on several repeated steps of vector addition and application of rotation matrices to transform from the global coordinate system, where for example tracks are defined in, to the coordinate system of the sector, followed by transformations to the module, the layer and finally to the cell. Such a general approach is acceptable for an offline system where the generality and ease of the method outweighs performance and system constraint considerations, as long as it is not a main bottleneck. 


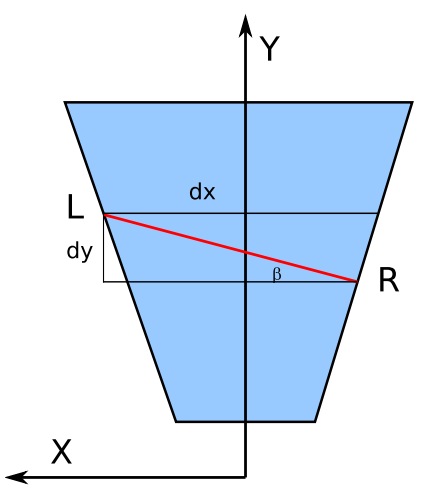

(a) drift cell inclination

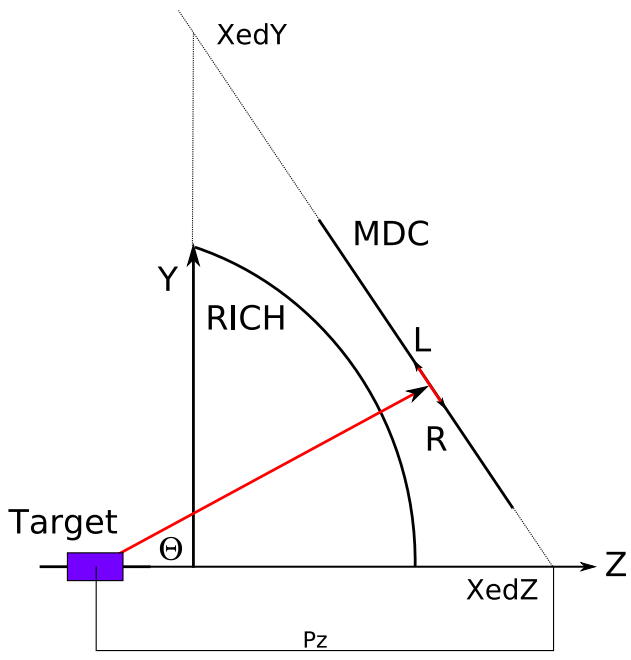

(c) layer base data and $\mathrm{z}$

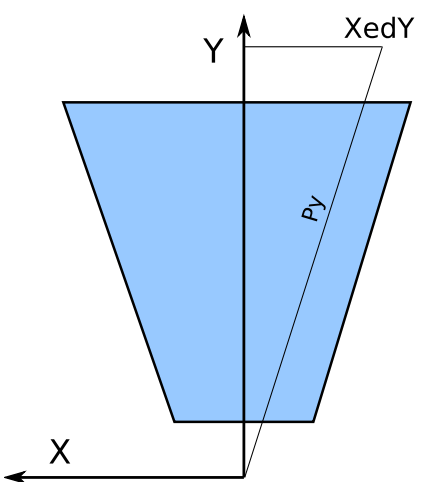

(b) projected layer Y

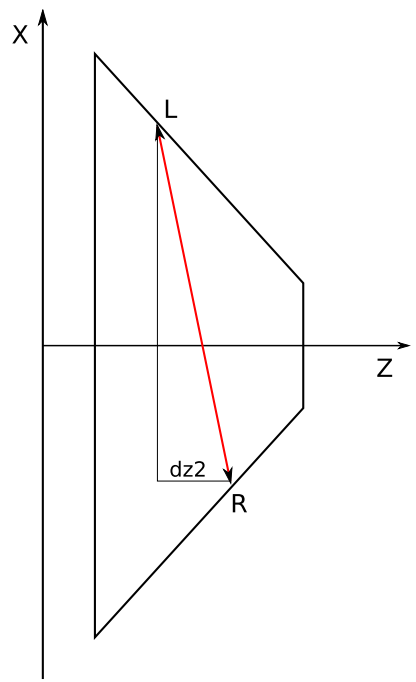

(d) z dep. of layer angle

Figure 4.2.: HADES Geometry for the Crossed Cell Calculation (calcXed).

The figures in (a) and (b) are views in beam direction, figure (c) is a view from the right side and $(\mathrm{d})$ is a view from above (all in arbitrary scales).

The terms in all figures are consistent with the naming scheme in the source code and have the following meaning: The left and right end points $(\mathrm{L} / \mathrm{R})$ of a sample MDC sense wire (red line) are shown and define the cell inclination angle $\beta$ for the MDC layer they belong to (shown in (a)). Figure (d) shows the correction for the z-coordinate depending on the $\mathrm{x}$-coordinate. The point XedY is located where a straight line in a MDC layer perpendicular to the drift cells of that plane (shown as Py in b) crosses the $\mathrm{x} / \mathrm{y}$-plane. XedZ is defined in a similar way as the point where the in-layer perpendicular line (Py) would cross the z-axis (see (b) and (c)). Figure (c) illustrates the shift of the target with respect to the source of the coordinate system and defines $\mathrm{Pz}$ and $\Theta$. 


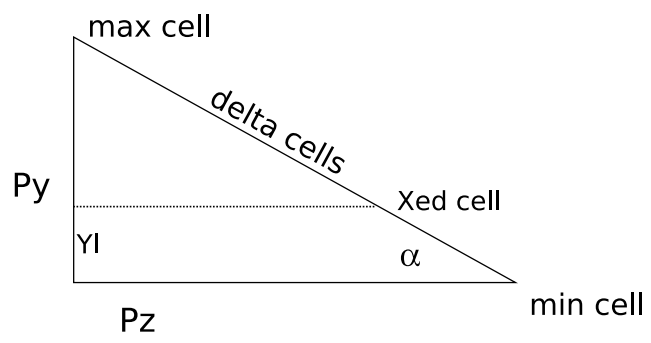

(a) cell from projected $\mathrm{Y}$

Figure 4.3.: Triangle for the last Step in the Calculation of the Crossed Cell.

This figure shows the relevant triangle for the calculation of the crossed drift cell. Its position in the HADES geometry and the definition of the variables can be found in Figure 4.2. The linear relation of Yl to crossed cell (Xed cell) is used to perform the calculation. Note that the value of max cell is greater than the existing number of cells. In the same way min cell is smaller than the lowest existing cell number, i.e. min cell is negative.

For the GE-MN with its limited system resources a different approach was chosen. The involved geometry transformation should be more direct and straightforward. The key idea is that all drift cells of a layer lie just parallel and next to each other in a plane, so that for a line perpendicular to the cells a linear relation between the coordinate and cell number exists. This and the following arguments can be followed when referring to Figure 4.3.

This is especially clear in case of the layers without inclination ( 3 and 4 ) in the module coordinate system (compare Figure 2.8 in Section 2.4), where the only relevant information to determine the drift cell hit is the y-coordinate. For the other layers an additional rotation around their inclination angle is required, and (when doing the final calculation) the changes in z caused by this (shown in Figure 4.2) have to be taken into account. The final calculation heavily relies on recalculated and tabled geometry data. The different parameters are described in the caption of Figure 4.2.

As a first step in implementing the calcXed() function the sixfold symmetry of HADES is used to rotate any RICH ring $(\vartheta, \phi)$ to sector 0 ( $\phi$ change only). This restricts all considerations to the same angular region (sector 0), simplifying the whole procedure (Equation (4.5)). The position information of the target is reduced to a shift in z-coordinate, the anyhow small $(\mathrm{x}, \mathrm{y})$ shifts are dropped as well as all considerations taking into account that the target is an extended object.

The next step is to calculate the tangent of the polar angle in this rotated system (Equation (4.6)), considering the corrected z coordinate. From this, the projected y-coordinate Yl (Equation (4.7)) is calculated. This determines the cell number cel by its linear dependence in the plane of the layers (Equation (4.8)). 
The function calcXed() executes the following algorithm:

$$
\begin{aligned}
s e c & =\text { alcSector }(\phi) \\
\phi_{s e c} & =\phi-\left(\sec \cdot 60 \cdot \frac{2 \pi}{360}\right)-\text { sectorCellAngleSML }[\text { sec }][\text { mod }][\text { lay }] \\
U_{x} & =\cos \left(\phi_{\text {sec }}\right) \\
U_{y} & =\sin \left(\phi_{\text {sec }}\right) \\
U_{z} & =\tan ^{-1}(\vartheta) \\
Z_{r e d} & =U_{z}+U_{x} \cdot \operatorname{tanZAlphaSML}[\text { sec }][\text { mod }][\text { lay }] \\
\tan \left(\vartheta^{\prime}\right) & =\frac{U_{y}}{Z_{\text {red }}} \\
\mathrm{Yl} & =\operatorname{PySML}[\text { sec }][\text { mod }][\text { lay }] /\left(1-\left(\tan \text { AlphaSML }[\text { sec }][\text { mod }][\text { lay }] / \tan \left(\vartheta^{\prime}\right)\right)\right) \\
\text { cel } & =\text { lineCellSMLmin }[\text { sec }][\text { mod }][\text { lay }]+\text { lineInclinSML }[\text { sec }][\text { mod }][\text { lay }] * \mathrm{Yl}
\end{aligned}
$$

Here, all the functions of the arguments [sec][mod][lay] are actually look-up tables containing precalculated values. The LUT sectorCellAngleSML $[\mathrm{sec}][\mathrm{mod}][\mathrm{lay}]$ stores the inclination angles indicated in Figure 4.2 as $\beta$. The LUT lineCellSMLmin [sec][mod][lay] stores the minimum cell number (min cell=offset) indicated in Figure 4.3 and lineInclinSML[sec][mod][lay] stores the linear factor between $Y_{l}$ and cel (compare Equation (4.8)). The LUT PySML[sec][mod][lay] stores the different Py values explained in Figure 4.2, likewise PzSML[sec][mod][lay] does the same for the values of $\mathrm{Pz}$. These lead to the definitions:

$$
\begin{gathered}
\text { tanAlphaSML }[\text { sec }][\text { mod }][\text { lay }]=-\frac{\operatorname{PySML}[\text { sec }][\text { mod }][\text { lay }]}{\operatorname{PzSML}[\text { sec }][\text { mod }][\text { lay }]} \\
\text { tanZAlphaSML }[\text { sec }][\text { mod }][\text { lay }]=-\frac{\mathrm{dz} 2}{\mathrm{dx} 2}
\end{gathered}
$$

The distance dz2 is shown in Figure 4.3 and dz2 is calculated as:

$$
\begin{aligned}
\mathrm{dz2}= & \left(\mathrm{L}_{x} / \cos (\text { sectorCellAngleSML }[\text { sec }][\bmod ][\text { lay }])\right) \\
& -\left(\mathrm{R}_{x} / \cos (\text { sectorCellAngleSML }[\text { sec }][\text { mod }][\text { lay }])\right)
\end{aligned}
$$

where $\mathrm{L}_{x}$ and $\mathrm{R}_{x}$ are the $x$ values of the points $\mathrm{L}$ and $\mathrm{R}$ in Figure 4.3. 


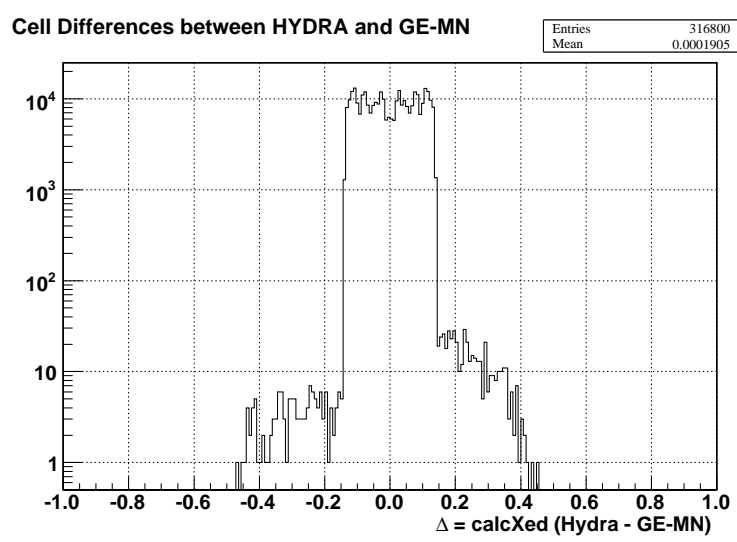

(a)

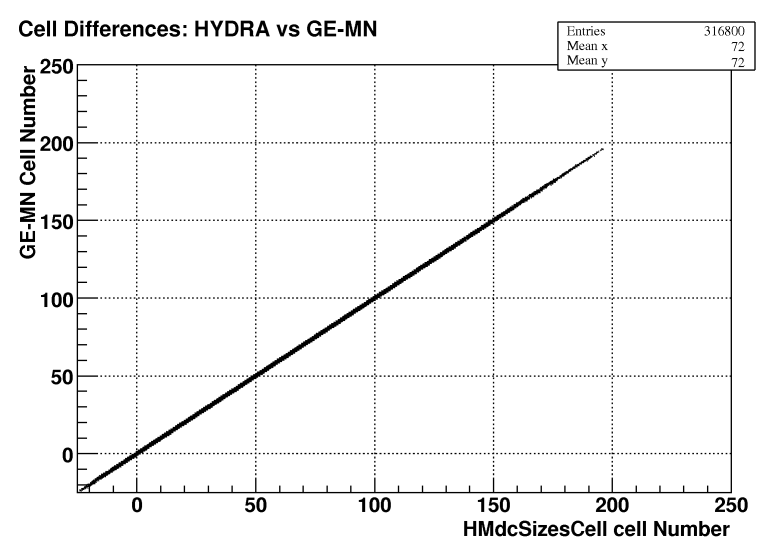

(b)

Figure 4.4.: Difference between calcXed() (GE-MN) and calcCrossedCells() (HYDRA).

Figure a) shows the difference in cell numbers, comparing the function integrated in the HYDRA framework (calcCrossedCells ()$)$ and the function used in the GE-MN (calcXed()). The used $(\vartheta ; \phi)$ - data was artificially chosen to scan the whole forward solid angle in small steps of one degree. Figure b) visualizes the difference in a $2 \mathrm{D}$ plot. Both plots show, that the discrepancies are very small and irrelevant for a trigger system using a matching window larger than a single cell, since $\Delta_{\max }<0,5$ cells here.

This method is efficient because most of the involved geometry calculations are performed in advance and the results are only retrieved from memory when needed. The amount of memory used to store precalculated geometry data is slightly below $3 \mathrm{KByte}$. This is much smaller than the "SMBT2LayCel_LUT" (see Equation (4.1)) which is 36 KByte large.

As a cross check of the accuracy of the calcXed() function its results have been compared with the calcCrossedCells() function integrated in the Hydra framework. This comparison can be found in Figure 4.4, which clearly shows that the accuracy is much better than needed, when the calcXed() function is used in a trigger application.

\subsubsection{Parameter Implementation in the GE-MN}

The data set stored in the different look-up tables is initially passed to the GE-MN by directly compiling it into the firmware of the GE-MN (see Section 3.3 and Section 3.1.4). This is accomplished by written the LUT data to a file, which is using the normal $\mathrm{C} / \mathrm{C}++$ source code syntax for initialized arrays, and as such can be just compiled with the other source code of the firmware. The firmware for the GE-MN contains a compiled-in and run specific default set of parameters in use.

\subsection{Correlation Results}

The correlation algorithm described in the previous sections has a different performance, depending on the input data used. To avoid continuously reoccurring descriptions and explanations of identical concepts a summarized description of the involved quantities and plots is presented 
here. Only the points of special interest for a certain data set/reaction are discussed in the sections of the individual results.

Since the discussed correlation method is tailored for online use, the first discussed plots are those which are immediately available online and thus allow to judge the behavior online. Examples of this are the RICH IPU hit distributions as well as the cell correlation plots (e.g. Figure 4.6) and the matched layers plot (e.g. Figure 4.8). This is followed by the discussion of quantities which can not be monitored online for several reasons. Discussions involving lepton efficiencies require the lepton track information, which itself needs a good detector calibration, the precise particle tracking and good high level particle identification. Since all of these are done in an involved iterative offline procedure these plots are not available online.

\subsubsection{RICH IPU Performance}

The RICH ring data samples (delivered by the RICH IPU) are not perfect [Leh00, Toi04]. They are largely fake dominated for several reasons. First, the RICH is not free of noise, and every noisy pad has a chance to contribute to a detected ring when combined with other fired pads. Those themselves can be noisy pads, or also real signal pads. In the latter case these pads belong to a real ring (reconstructable ring caused by a real lepton), which means that there is the possibility to form an other ring candidate with a noisy pad and a part of the ring circumference. This kind of noise is correlated to real rings. In addition, it happens that charged particles travel through the back-plane and create strong ionization at the pads.

One source of noisy pads in the RICH is the always present electronic noise, pronounced by low threshold settings intended to increase the photon detection efficiency. The other main contribution is due to charged particles traveling though the $\mathrm{RICH}$, other than the primary reaction leptons whose detection is intended. For example a certain part of the $\delta$-electrons (knock-out electrons) has energies high enough to produce ČERENKOV light, and thus to produce fired pads. Example histograms discussing the RICH performance can be found in Figure 4.5.

\subsubsection{Trigger Performance Parameters}

The performance of trigger algorithms in general and the correlation method discussed here in particular can be characterized by three different parameters: Reduction, efficiency and enhancement, as defined in the following equations:

$$
\begin{aligned}
\text { Reduction: } & \text { red }=\frac{\text { Accepted Events }}{\text { Total Events }} \\
\text { Efficiency: } & \text { eff }=\frac{\text { Accepted and good Events }}{\text { Total good Events }} \\
\text { Enhancement: } & \text { enh }=\frac{\text { Efficiency }}{\text { Reduction }}
\end{aligned}
$$

The reduction describes which fraction of the events is accepted, and thus also gives the reduction of the event rate. The efficiency describes the fraction of the good events which are accepted, thus $(1-$ eff) is the rate of false negative decisions which are irrevocably loosing good data (see below). 
Finally, the enhancement describes the enrichment of good events in the accepted data sample. For the purpose of this work good events are those that contain good leptons as defined in Section 4.1.2.

Calculating efficiency and enhancement involves knowing which events are good events and implicitly which good events are rejected by the trigger. This can only be investigated with the so called downscaled events. These are events which are recorded independently of the decision of the trigger method, while still recording the trigger decision. The full offline analysis of the downscaled events reveals which are the good events and which trigger decision has been taken for them.

\begin{tabular}{|c|c|c|}
\hline Decision Type & Trigger Decision & Data Sample \\
\hline \hline correct positive & accept & contains good data \\
\hline correct negative & reject & does not contain good data \\
\hline false positive & accept & does not contain good data \\
\hline false negative & reject & contains good data \\
\hline
\end{tabular}

Table 4.2.: Trigger Decision Types.

A trigger algorithm can either accept an event or reject it, while the event can contain either interesting (good) data or not. Thus one of the four possible cases occurs per event as summarized in Table 4.2. A correct positive or correct negative decision is the intended behavior of the algorithm. False positive decisions reduce the reduction, but do not discard interesting data, as there is no interesting data in the event. Also no physics bias is introduced, if these events are properly treated (rejected/tagged) by a more sophisticated offline analysis. False negative events need a more careful treatment. Since these are discarded, there is afterwards no way of telling if good data samples were lost and to which amount.

Of even higher importance is the situation if false negative decisions occur significantly more often at certain parameters. For example it would clearly introduce a bias into the physics derived from an experiment, if there would be a significant excess of false negative decisions at a certain momentum. False negative decisions are only acceptable if they occur with the same probability to all types of events. A way to check this is to investigate the efficiency as function of another parameter, as it is done in Section 4.4.5, Section 4.4.6 and Section 4.4.7. There, the angular efficiency distributions $\operatorname{eff}(\phi)$ and $\operatorname{eff}(\vartheta)$ as well as the momentum distribution $\operatorname{eff}(p)$ are investigated, and all of them should be flat in the region of interest.

\subsubsection{Online Analysis Considerations}

Available online are the RICH IPU distributions for $\phi, \vartheta$ and the ring multiplicity. These are interesting since these data samples are the starting point for the correlation method. Noisy spots in the RICH can be identified there (e.g. Figure 4.5), and it can be crosschecked by comparing them to non noisy regions, if these have an impact on the correlation.

Also available online are the correlation histograms of calculated crossed cells (from function calcXed ()$)$ to the real hit MDC cells. If the correlation is working properly a clear peak at zero is visible even with low statistics. The width of this peak enables the determination and monitoring of the usable settings of the width of the matching window. These correlation histograms are also available for the individual sectors, making it possible to detect if there is a problem in a single sector. 
To improve the speed and ease of use of the analysis a special array is filled. It contains as each of its 12 entries the minimum matching window size necessary to match the event with the required number of layers, which is given by the index into the array. This array of the name deltaMinSortEvenLay12[] is filled for every event. This makes it possible to query if an event is accepted with certain correlation parameters by checking the BOOLEAN expression:

$$
\text { deltaMinSortEvenLay12 }\left[L_{M}\right]<M_{W}
$$

This array is filled when calculating the difference of the calculated hit cell number and the number of the actual hit cells. The minimum of the cell differences is the minimum matching window size necessary to positively match this layer. The calculation is performed for each layer, and the result is filled into an array with a capacity of twelve elements. For layers without real MDC hits a big number, larger than the maximum cell difference is entered into the array (i.g. $444,444)$. Sorting the entries of the arrays in ascending order gives one deltaMinSortRingLay12 [ array per RICH IPU ring. Finding the minimum cell difference $(=$ minimum required matching window) per layer in these per RICH IPU ring arrays gives deltaMinSortEvenLay12[.

The next type of histogram available online is the distribution of the number of matched layers with a given matching window (e.g. Figure 4.8). This histogram should always show a peak in the zero bin for all the RICH IPU rings which could not matched at all and a more or less smooth rise to higher numbers of matched layers. This plot enables a quantitative measure if the number of layers required inside the matching window could be higher or is already too high. An always conservative setting of the number of required layers parameter is 4 , since the cluster finder used in the offline analysis will not find a track in this case anyway (compare Section 4.2.1).

\subsubsection{Offline Analysis Considerations}

All other plots are available offline only (or in the best case semi-online as the so called online $\mathrm{DST}^{1}$ ). The reason is, that the plots involve good leptons. To identify the good Leptons the full track reconstruction is required. The identification of good leptons for the purpose of this work is discussed in Section 4.1.2. The first set of offline plots are the plots just explained in Section 4.4.3, but redone showing only the identified good leptons. These plots show the influence of the correlation trigger on the events containing the good leptons.

Ideally, the correlation histograms (e.g. Figure 4.7) would show a clear, sharp peak at zero and no events left or right. The events left and right of the peak, and outside the correlation window, are those where the decision is false negative. Also the histograms showing the distribution of the number of matched layers are interesting. It shows a broad peak centered at a high number of layers (e.g. Figure 4.9) for the good leptons. Entries below the required matched number of layers are false negatives. Thus this histogram shows the theoretical, optimum setting of the required layers parameter. Both previously deduced trigger parameters should be compared to the distributions in these two histograms.

For a thorough investigation which correlation parameters yield a nice reduction and enhancement, while still having a high efficiency, a systematic scan of different correlation parameters is presented as a table (e.g. Table 4.3). The table shows the results of eff (Efficiency) and enh (Enhancement) for different settings of the required layers (Layers) and matching window (Window). The entry named L2 shows if the HADES second level trigger (compare Section

\footnotetext{
${ }^{1}$ online DST: Running the DST immediately in parallel to the experiment. The results are not perfectly calibrated and available a couple of hours after taking the data.
} 
2.12) was active for this setting, while L3 denotes the same for the correlation method discussed here. The table shows that the individual performances are checked as well as how both systems perform combined. The Multiplicity (M) column states the minimum number of positive trigger decisions per event required. Therefore, multiplicity 1 stands for a single lepton trigger while multiplicity 2 stands for a dilepton trigger setting. Efficiency two (eff2) and enhancement two (enh2) calculate the efficiency and the enhancement only for the events which have a RICH IPU ring and MDC fired wires in the same sector, i.e. they can be correlated at all. This shows the strong influence of the efficiency of the RICH IPU on the results of the correlation, and turns out to be the dominant factor. Result number 0 shows the performance of the HADES second level trigger for this data set for comparison. Finally, the captions of the tables for the individual data sets elaborate on which combinations of settings are useful.

Most interesting in the offline analysis is how the correlation method changes distributions and results for good leptons. This can be investigated by comparing different good lepton distributions. For example, the comparison of the $\vartheta, \phi$ and momentum (e.g. Figure 4.11, Figure 4.12, Figure 4.13) distributions versus the same distributions for the good leptons which passed the correlation trigger. Shown are (for the trigger settings identified as useful before) the distributions of angular efficiencies $\operatorname{eff}(\phi), \operatorname{eff}(\vartheta)$ and momentum efficiency distribution eff $(p)$. If the correlation trigger would work perfectly the result would be a straight line at an efficiency equal to one. In reality, the efficiencies are smaller that one, but should still be flat for the usable trigger settings.

The discussion of the individual interesting features per data set can be found in the captions where they are visible. Section 5.2 compares them and discusses general features and results. 


\subsubsection{Correlation Analysis - $\mathbf{p}+\mathbf{p}$ at $2,2 \mathrm{GeV}$}

All the presented figures in this section are derived from one experimental run of the January 2004 beamtime. This ensures consistency by making sure that not several calibration parameters are mixed into the same analysis. The target was a $5 \mathrm{~cm}$ thick liquid hydrogen target and the beam energy was $2,2 \mathrm{GeV}$. The generation 3 DST macros and parameters are used.
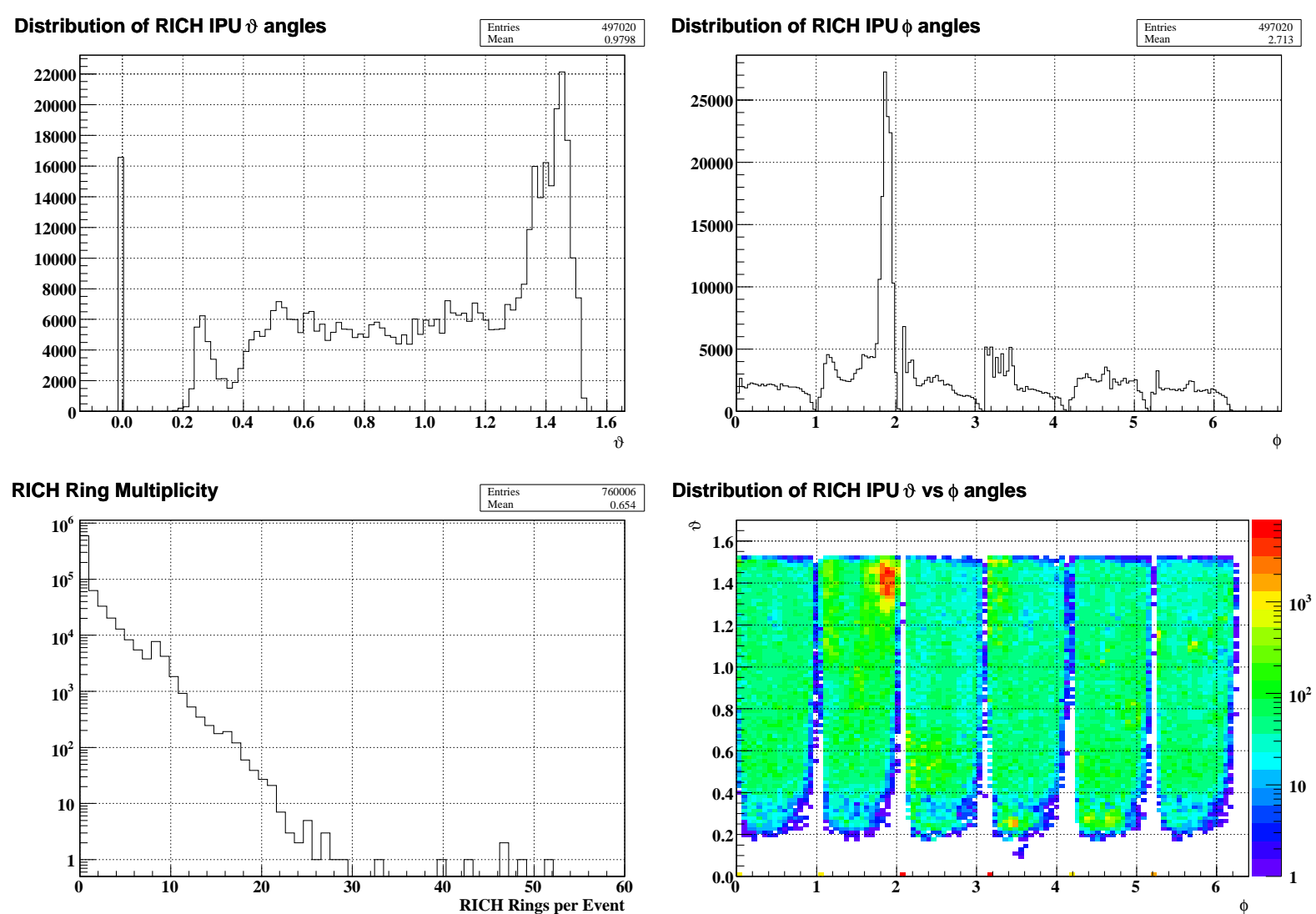

Figure 4.5.: p + p@2,2 GeV: RICH Ring Distribution from IPU Data (available online).

The histograms show the distributions of the RICH rings as they are reconstructed by the RICH IPU (compare Figure 2.4). The bottom left histogram shows the RICH IPU ring multiplicity per event. The six different sectors are visible in the $\phi$-distribution and are numbered from left to right as 5, 0, 1, 2, 3, 4 (definition of coordinate system: Figure 2.1). Prominent in sector 0 is the noise peak between $\phi=1,6$ and $\phi=2,0$. The noisy RICH region is also visible in the $\vartheta$-distribution as a single peak on the right side of sector 0 . The two dimensional histogram shows $\vartheta$ versus $\phi$. It shows where exactly the noise is localized in one specific region of the RICH, visible as a clear hot spot in the logarithmic color coded diagram. This hot spot should be kept in mind when investigating the next figures, since it is discussed there which influence this hot spot has. 

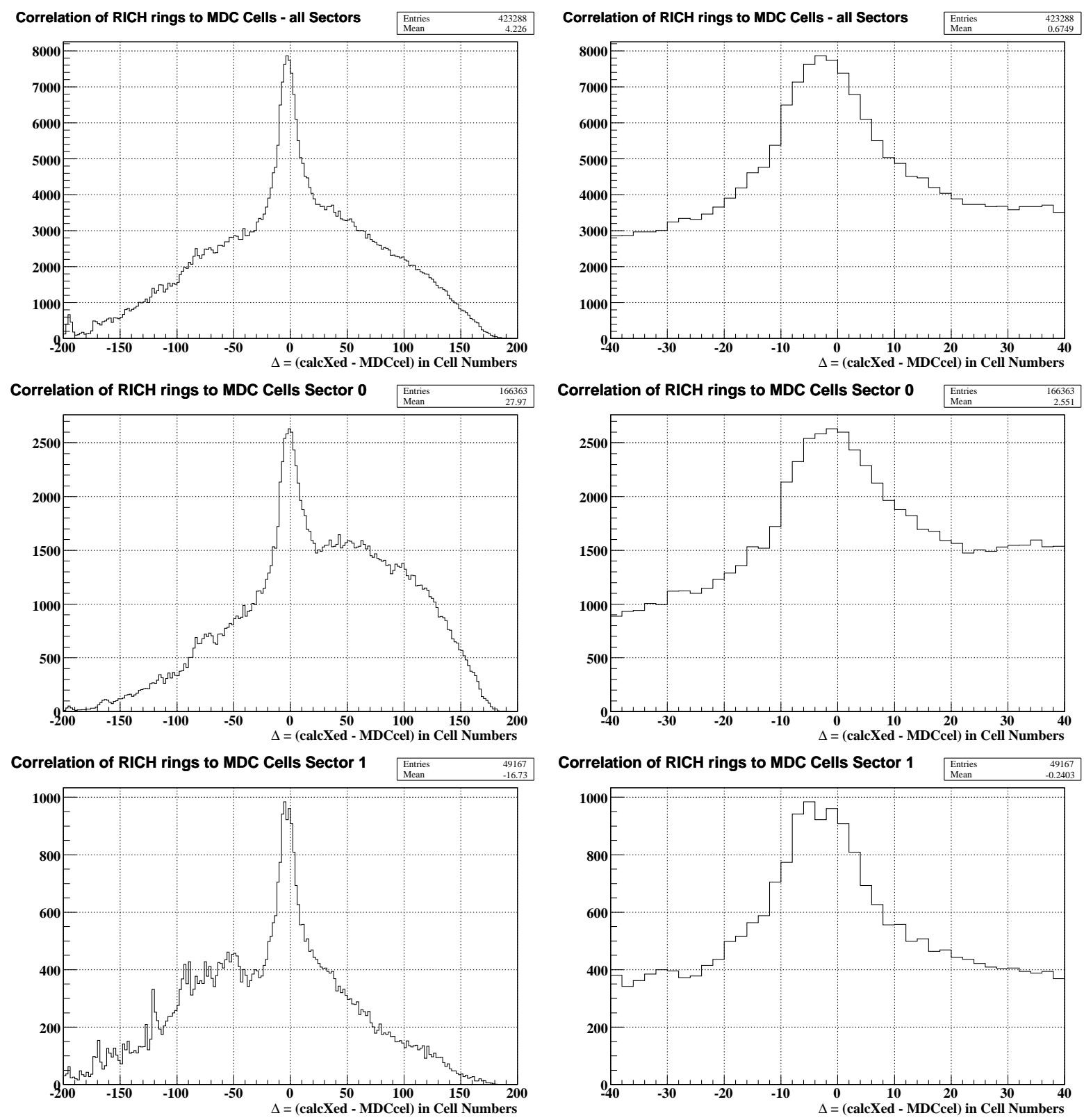

Figure 4.6.: $p+p @ 2,2 \mathrm{GeV}$ : RICH IPU Ring to MDC Cell Correlation (available online).

The histograms on the right show a magnified view of the correlation histograms on the left. The presence of a clear peak at 0 shows that the correlation is working, and the width of the correlation peak allows to decide which correlation window is safe to use. The noise peak in the RICH IPU data in sector 0 (compare Figure 4.5) creates the shoulder on the right side of the correlation peak, which is absent in the other sectors, as seen in the example of sector 1 . A safe window is 20 cells, while a more aggressive (but still realistic) cut is 10 cells. The direct comparison with Figure 4.7 shows how these cuts affect the accepted good leptons. 

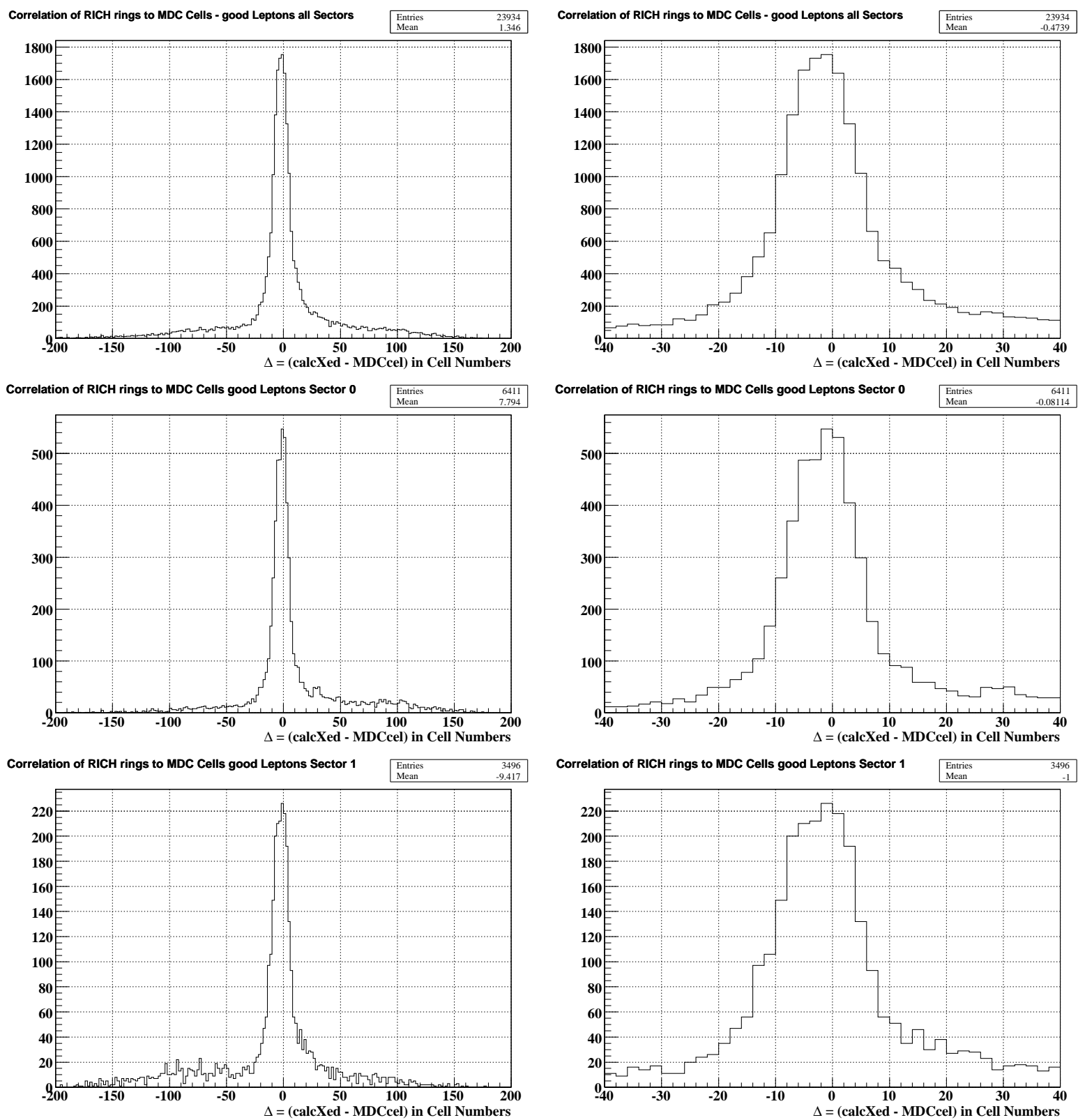

Figure 4.7.: $\mathbf{p}+\mathrm{p} @ 2,2 \mathrm{GeV}$ : RICH IPU Ring to MDC Cell Correlation for good Leptons.

The histograms on the right side show a magnified view of the correlation histograms on the left. The presence of a clear peak at 0 shows that the correlation is working well for the good leptons, and the width of the correlation peak shows how narrow the matching window cut could be for the good leptons to be accepted. The noisy shoulder in sector 0 is gone here as it is not caused by any good lepton, but just caused by the noise in the RICH IPU data. Comparing the width of these histograms with the width deduced from Figure 4.6 it is clear, that a cut of 20 cells is really safe and a cut with a width of down to 10 cells is still realistic. 

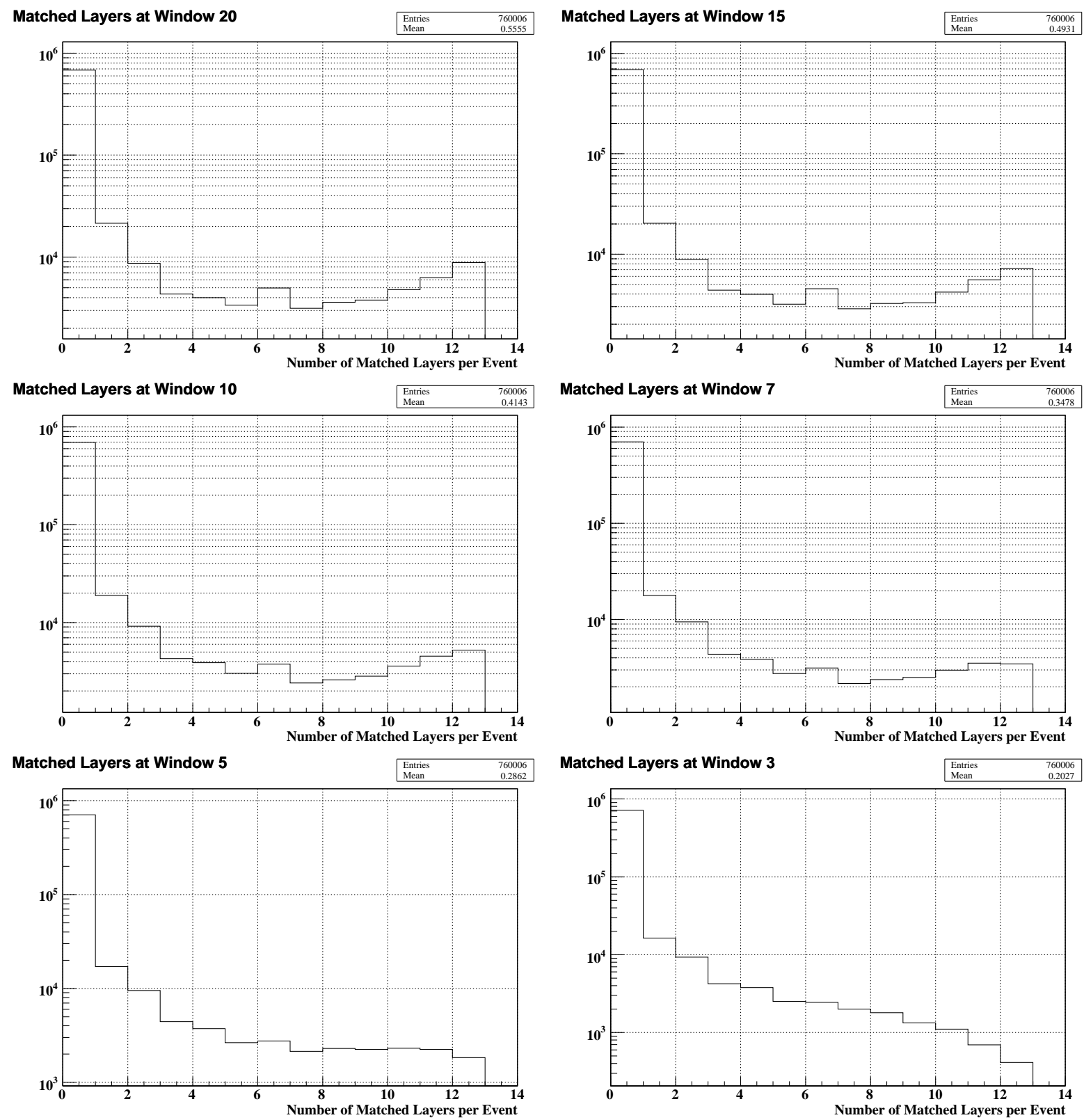

Figure 4.8.: p + p@2,2 GeV: Matched Layers Distributions (available online).

The histograms show how many layers can be matched for different settings of the width of the correlation window. Events with no correlation (or little/random) end up in the bins to the left. Interesting to note is the rise of the distributions at higher numbers of matched layers, as long as the matching window is still large enough $(\geqq 7)$. It can be seen here that smaller matching windows are not useful, because they start to cut into the correlation rise at higher layer numbers. The save cut is at 4 layers (compare Section 4.2.1). Assuming that correlated leptons have a larger number of matched layers, one can look at the rise on the right and place the cut before it starts. This means for the just deduced window cuts of 20 cells, and 10 cells layer cuts at 8 layers and 6 layers respectively. A very aggressive cut would be 10 layers. These cuts should be compared to the histograms in Figure 4.20 to check the effect of this layer cut onto the accepted good leptons. 

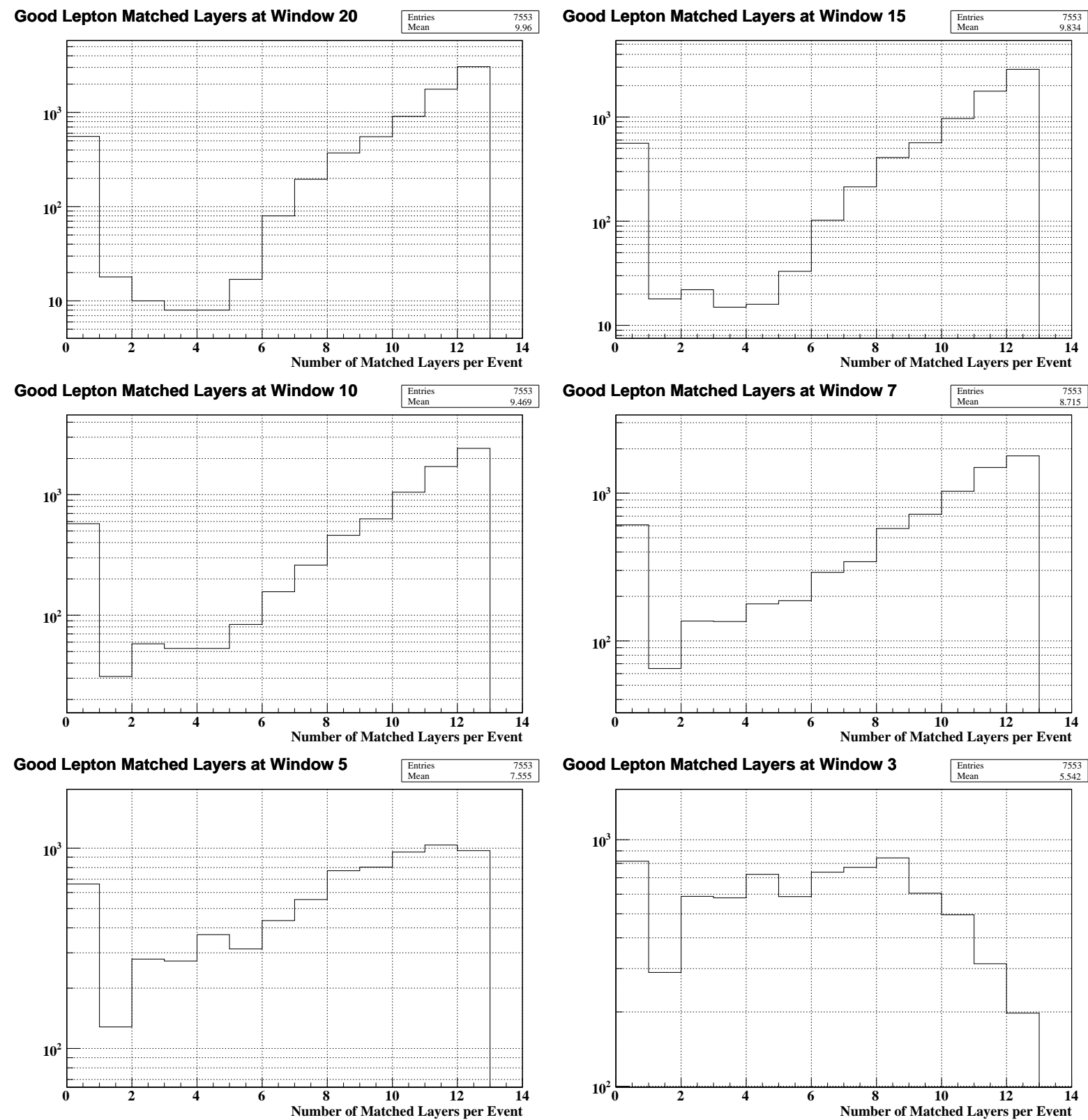

Figure 4.9.: p + p@2,2 GeV: Matched Layers Distributions for good Lepton Events.

The Figures show the number of matched layers distributions for different settings of the matching window. These histograms show only events with good leptons. The strong rise towards a higher number of matched layers for these good events is clearly visible, as well as the valley around 4 matched layers for moderate window sizes (20 to 10). It should be noted that the bin at zero matched layers is fairly constant for different settings. Comparing these histograms with the just deduced minimum number of layers cuts it is clearly visible that a cut at 6 layers is always before the rise and a cut at 8 layers starts to cut just into the rise of the correlated good leptons. A cut at 10 layers is already cutting into the correlated leptons. The influence of a too small matching window is also visible, as in these cases the distributions get largely smeared out. 
Trigger Analysis for run:

January 2004

\begin{tabular}{|c|c|c|c|c|c|c|c|c|c|c|}
\hline Result & L2 & $\mathbf{L 3}$ & $\mathbf{M}$ & Layers & Window & red & eff & eff 2 & enh & enh2 \\
\hline 0 & Yes & No & 1 & n.a. & n.a. & 0,073 & 0,781 & n.a. & 10,728 & n.a. \\
\hline 1 & No & Yes & 1 & 4 & 20 & 0,056 & 0,922 & 0,982 & 16,387 & 17,455 \\
\hline 2 & No & Yes & 1 & 4 & 15 & 0,050 & 0,918 & 0,978 & 18,380 & 19,578 \\
\hline 3 & No & Yes & 1 & 4 & 10 & 0,042 & 0,905 & 0,964 & 21,594 & 23,001 \\
\hline 4 & No & Yes & 1 & 4 & 7 & 0,035 & 0,875 & 0,932 & 24,866 & 26,486 \\
\hline 5 & No & Yes & 1 & 4 & 5 & 0,029 & 0,822 & 0,876 & 28,132 & 29,964 \\
\hline 6 & No & Yes & 1 & 6 & 20 & 0,047 & 0,918 & 0,978 & 19,727 & 21,012 \\
\hline 7 & No & Yes & 1 & 6 & 15 & 0,041 & 0,912 & 0,971 & 22,487 & 23,952 \\
\hline 8 & No & Yes & 1 & 6 & 10 & 0,033 & 0,887 & 0,945 & 27,030 & 28,791 \\
\hline 9 & No & Yes & 1 & 6 & 7 & 0,026 & 0,827 & 0,880 & 31,214 & 33,248 \\
\hline 10 & No & Yes & 1 & 6 & 5 & 0,021 & 0,732 & 0,779 & 35,123 & 37,412 \\
\hline 11 & No & Yes & 1 & 8 & 20 & 0,036 & 0,882 & 0,940 & 24,575 & 26,176 \\
\hline 12 & No & Yes & 1 & 8 & 15 & 0,031 & 0,870 & 0,927 & 28,195 & 30,032 \\
\hline 13 & No & Yes & 1 & 8 & 10 & 0,025 & 0,831 & 0,886 & 33,650 & 35,842 \\
\hline 14 & No & Yes & 1 & 8 & 7 & 0,020 & 0,743 & 0,791 & 38,040 & 40,518 \\
\hline 15 & No & Yes & 1 & 8 & 5 & 0,014 & 0,601 & 0,640 & 41,794 & 44,517 \\
\hline 16 & No & Yes & 1 & 10 & 20 & 0,026 & 0,760 & 0,809 & 29,028 & 30,919 \\
\hline 17 & No & Yes & 1 & 10 & 15 & 0,022 & 0,741 & 0,790 & 33,209 & 35,373 \\
\hline 18 & No & Yes & 1 & 10 & 10 & 0,018 & 0,687 & 0,732 & 39,089 & 41,636 \\
\hline 19 & No & Yes & 1 & 10 & 7 & 0,013 & 0,571 & 0,608 & 43,609 & 46,451 \\
\hline 20 & No & Yes & 2 & 4 & 20 & 0,029 & 0,690 & 0,753 & 24,007 & 26,210 \\
\hline 21 & No & Yes & 2 & 4 & 15 & 0,025 & 0,687 & 0,750 & 27,674 & 30,213 \\
\hline 22 & No & Yes & 2 & 4 & 10 & 0,020 & 0,641 & 0,699 & 32,795 & 35,805 \\
\hline 23 & No & Yes & 2 & 4 & 7 & 0,015 & 0,574 & 0,627 & 38,789 & 42,348 \\
\hline 24 & No & Yes & 2 & 6 & 20 & 0,023 & 0,687 & 0,750 & 30,240 & 33,015 \\
\hline 25 & No & Yes & 2 & 6 & 15 & 0,019 & 0,664 & 0,725 & 34,557 & 37,729 \\
\hline 26 & No & Yes & 2 & 6 & 10 & 0,014 & 0,600 & 0,655 & 41,444 & 45,247 \\
\hline 27 & No & Yes & 2 & 6 & 7 & 0,010 & 0,510 & 0,557 & 48,923 & 53,413 \\
\hline 28 & No & Yes & 2 & 8 & 20 & 0,018 & 0,635 & 0,693 & 36,068 & 39,378 \\
\hline 29 & No & Yes & 2 & 8 & 15 & 0,015 & 0,612 & 0,668 & 41,665 & 45,489 \\
\hline 30 & No & Yes & 2 & 8 & 10 & 0,011 & 0,519 & 0,566 & 49,284 & 53,807 \\
\hline 31 & No & Yes & 2 & 8 & 7 & 0,007 & 0,374 & 0,408 & 56,328 & 61,498 \\
\hline 32 & No & Yes & 2 & 10 & 20 & 0,011 & 0,513 & 0,560 & 45,770 & 49,971 \\
\hline 33 & No & Yes & 2 & 10 & 15 & 0,009 & 0,467 & 0,509 & 51,943 & 56,710 \\
\hline 34 & No & Yes & 2 & 10 & 10 & 0,006 & 0,354 & 0,386 & 61,627 & 67,283 \\
\hline 35 & No & Yes & 2 & 10 & 7 & 0,003 & 0,223 & 0,244 & 73,526 & 80,274 \\
\hline 36 & Yes & Yes & 1 & 4 & 20 & 0,033 & 0,776 & 0,826 & 23,385 & 24,908 \\
\hline 37 & Yes & Yes & 1 & 4 & 15 & 0,030 & 0,773 & 0,824 & 25,533 & 27,196 \\
\hline 38 & Yes & Yes & 1 & 4 & 10 & 0,026 & 0,762 & 0,812 & 28,934 & 30,819 \\
\hline 39 & Yes & Yes & 1 & 4 & 7 & 0,023 & 0,736 & 0,784 & 32,285 & 34,388 \\
\hline 40 & Yes & Yes & 1 & 6 & 20 & 0,029 & 0,773 & 0,823 & 26,800 & 28,546 \\
\hline 41 & Yes & Yes & 1 & 6 & 15 & 0,026 & 0,768 & 0,818 & 29,726 & 31,662 \\
\hline 42 & Yes & Yes & 1 & 6 & 10 & 0,022 & 0,748 & 0,797 & 34,427 & 36,670 \\
\hline 43 & Yes & Yes & 1 & 6 & 7 & 0,018 & 0,694 & 0,740 & 38,501 & 41,009 \\
\hline 44 & Yes & Yes & 1 & 8 & 20 & 0,023 & 0,742 & 0,791 & 32,011 & 34,097 \\
\hline 45 & Yes & Yes & 1 & 8 & 15 & 0,021 & 0,734 & 0,782 & 35,713 & 38,039 \\
\hline 46 & Yes & Yes & 1 & 8 & 10 & 0,017 & 0,701 & 0,747 & 41,182 & 43,866 \\
\hline 47 & Yes & Yes & 1 & 8 & 7 & 0,014 & 0,621 & 0,661 & 45,202 & 48,147 \\
\hline 48 & Yes & Yes & 1 & 10 & 20 & 0,018 & 0,640 & 0,682 & 36,225 & 38,585 \\
\hline 49 & Yes & Yes & 1 & 10 & 15 & 0,015 & 0,625 & 0,665 & 40,498 & 43,137 \\
\hline 50 & Yes & Yes & 1 & 10 & 10 & 0,013 & 0,577 & 0,614 & 46,076 & 49,078 \\
\hline 51 & Yes & Yes & 1 & 10 & 7 & 0,010 & 0,478 & 0,509 & 50,263 & 53,538 \\
\hline
\end{tabular}

Table 4.3.: $\mathbf{p}+\mathbf{p} @ 2,2 \mathrm{GeV}$ : Efficiency and Enhancement for different Correlation Parameters.

The table shows a systematic calculation of efficiency and enhancement for different settings of the correlation parameters. The L3 column denotes if the RICH to MDC correlation trigger was active, while L2 denotes the same for the HADES level 2 trigger system. Multiplicity (M) is the minimum number of required good leptons per event. Result 0 shows the behavior of the HADES level 2 trigger for the same data set for comparison. From this table and the discussion of the previous distributions a particular useful trigger setting would be for example 6 layers and 10 cells (Result: 8). This parameter set gives a good enhancement while still having a reasonable efficiency. This set should be cross referenced with the Efficiency distributions in Figure 4.11, Figure 4.12 and Figure 4.13. The values of eff2 and enh2 demonstrate the influence of the RICH IPU (compare Section 4.4.4). 

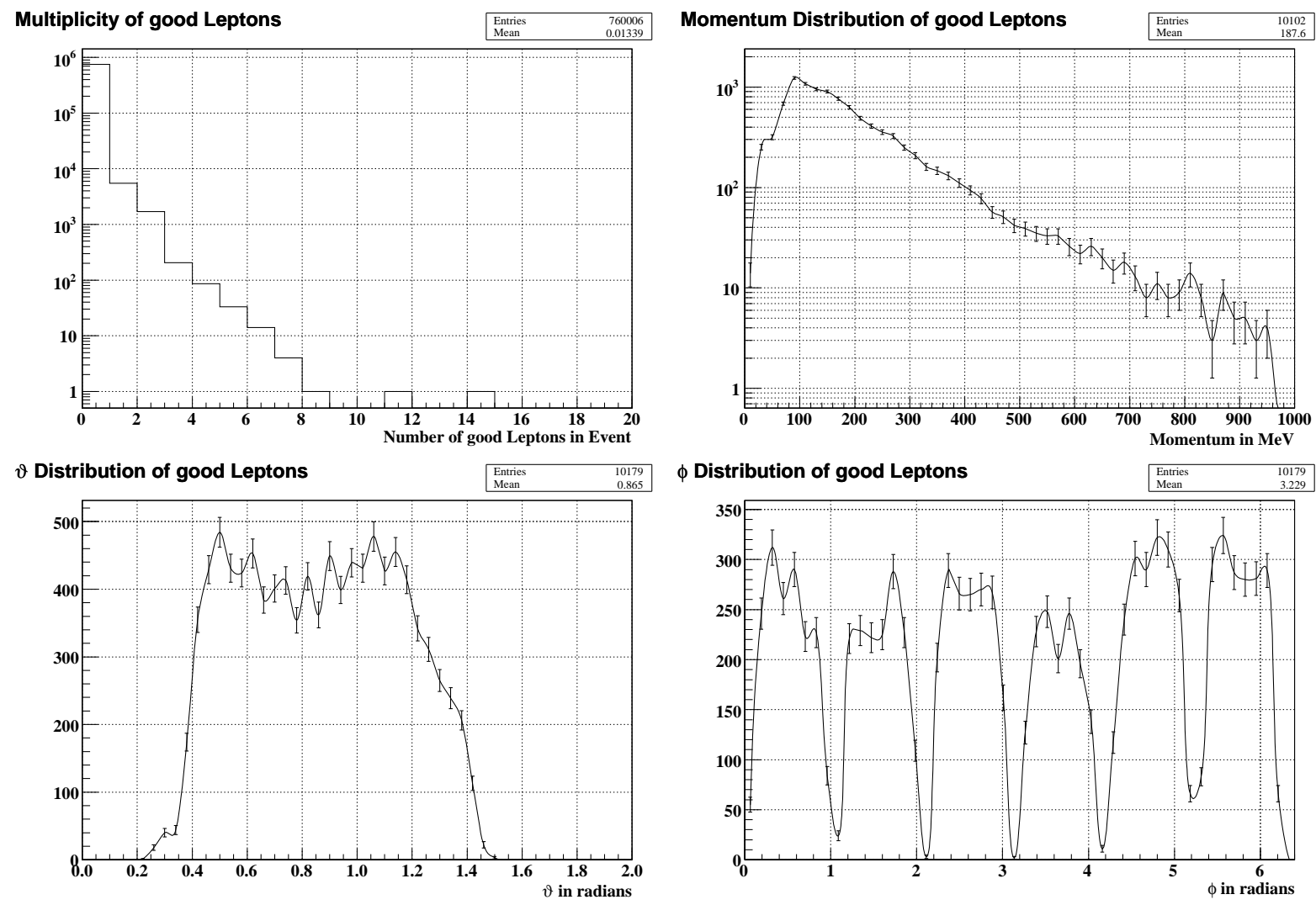

Figure 4.10.: p + p@2,2 GeV: Good Lepton Base Data.

The histograms show the base data of the good leptons. Their multiplicity per event histogram shows how few events actually contain one or more good lepton. The $\vartheta$-distribution and the $\phi$ distribution show the respective angles of the lepton before the magnetic field, and clearly follow the geometrical structure of the HADES detector. The momentum distribution is obtained from the "spline" algorithm, using the bending of the track in the magnetic field. These distributions provide the good total events in Equation (4.10). Dividing these histograms with the accepted good lepton distributions for different correlation parameters leads to the different efficiency distribution histograms. The errors are deduced from the statistical errors only. 

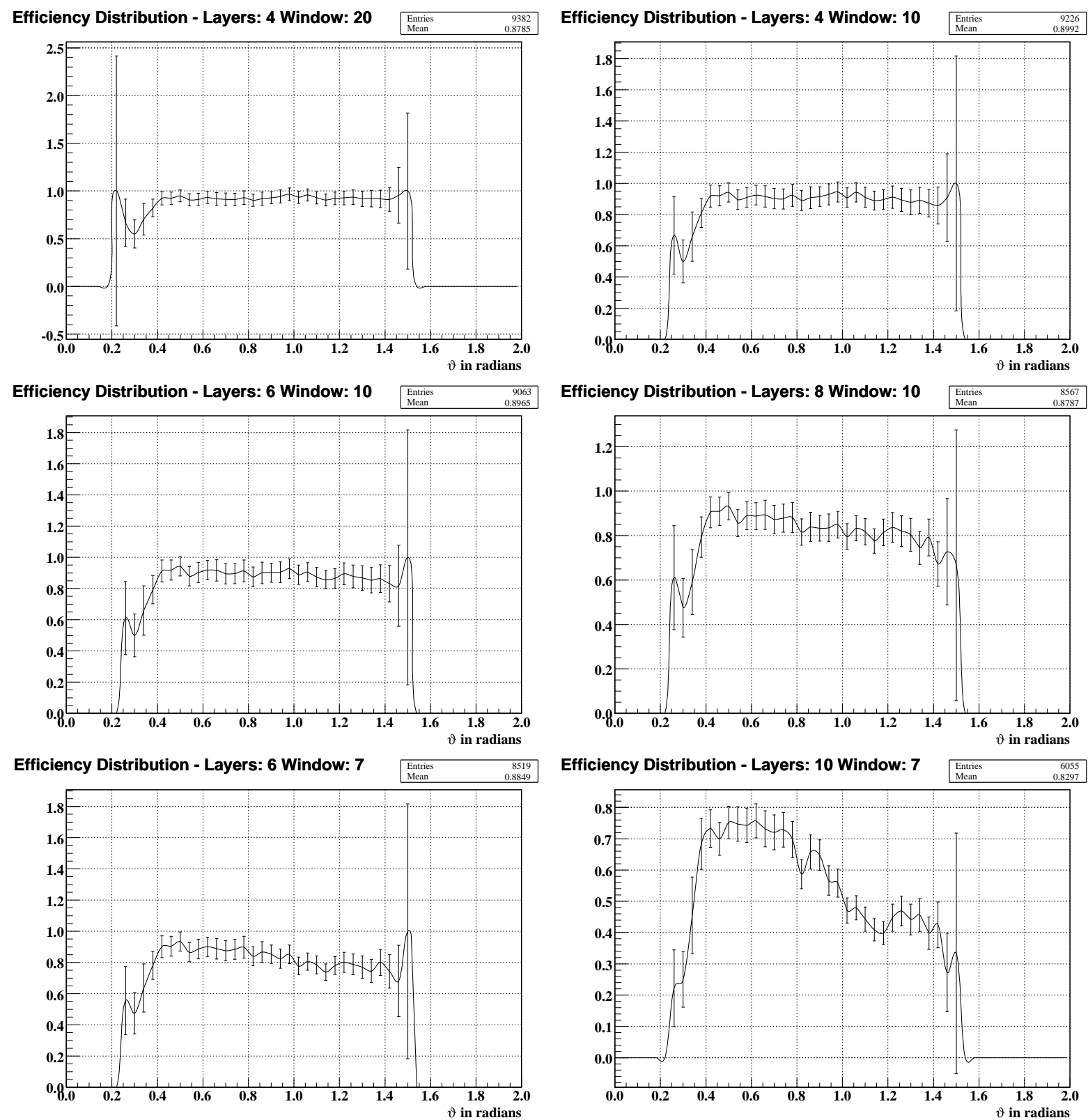

Figure 4.11.: $\mathbf{p}+\mathbf{p} @ 2,2 \mathrm{GeV}: \vartheta$ - Efficiency Distributions

These histograms show the efficiency as a function of the $\vartheta$-angle eff $(\vartheta)$ as defined in Section 4.4.2 for several selected settings of the correlation algorithm. The first three distributions are flat, except for the edges of the chambers where the limited statistics prevents the results from being very meaningful. Starting from there, it is visible that with more aggressive trigger parameters the distribution starts to drop at higher $\vartheta$ - angles and even develops a strong bump in diagram 10 layers and 7 cells. 
Efficiency Distribution - Layers: 4 Window: 20

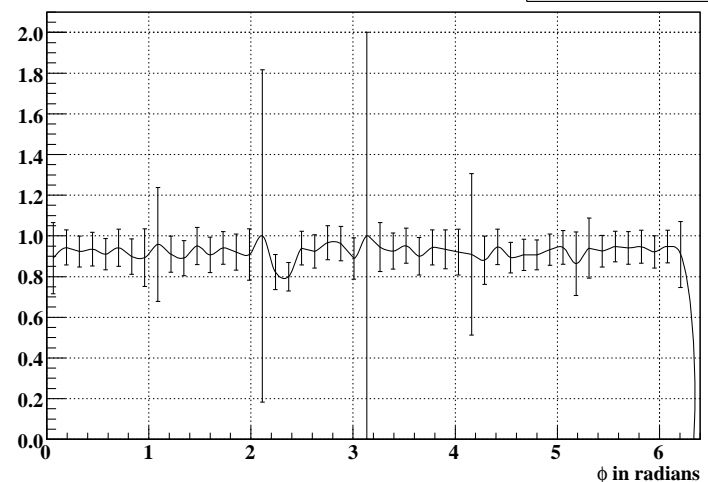

Efficiency Distribution - Layers: 6 Window: 10

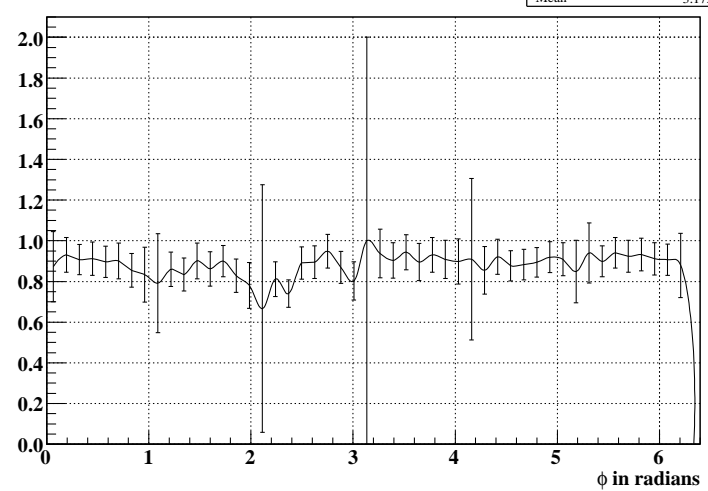

Efficiency Distribution - Layers: 6 Window: 7

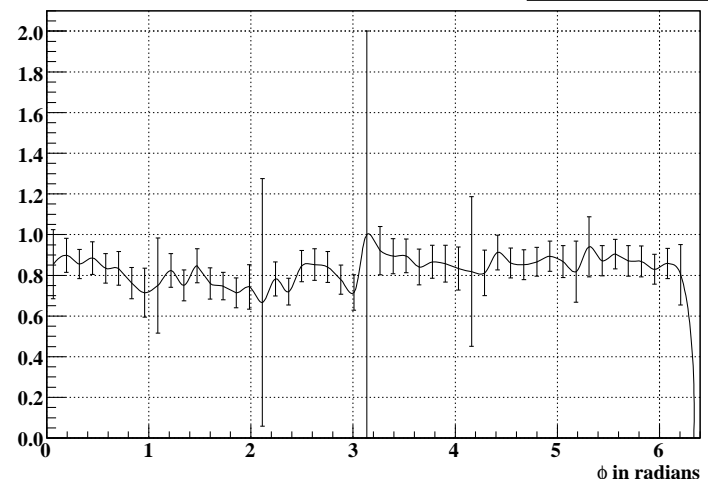

Efficiency Distribution - Layers: 4 Window: 10

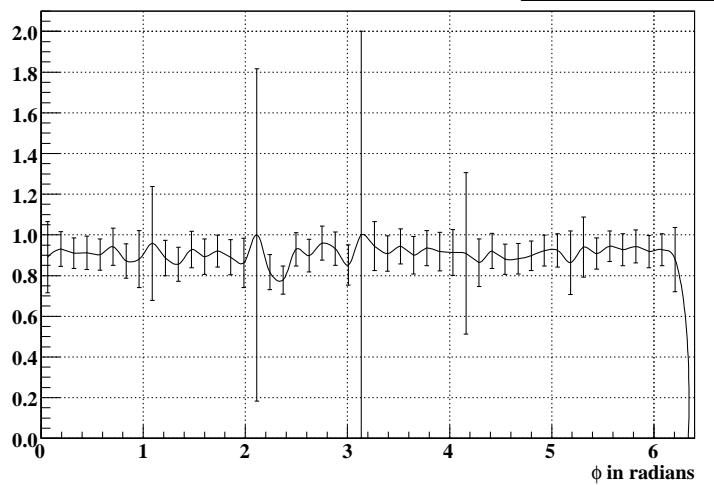

Efficiency Distribution - Layers: 8 Window: 10

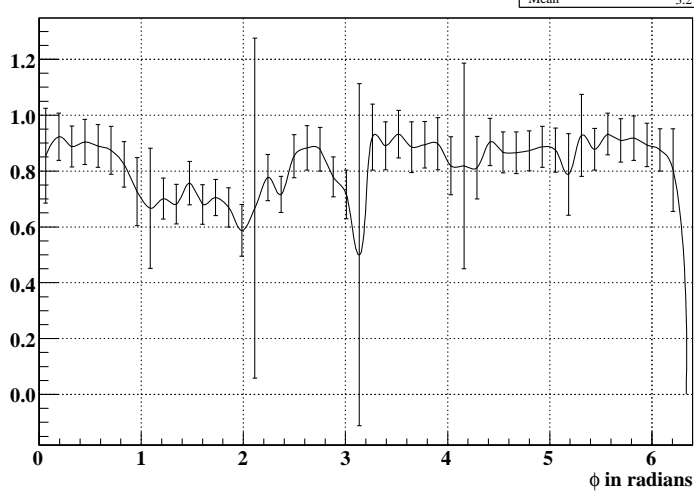

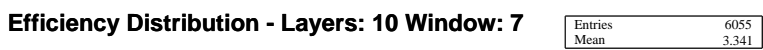

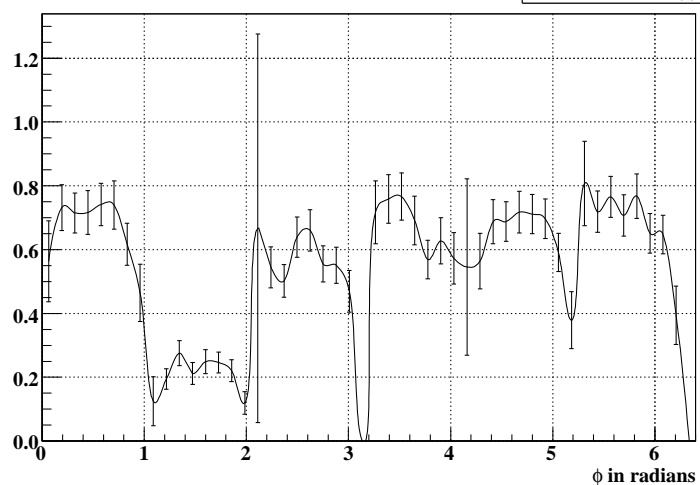

Figure 4.12.: $\mathbf{p}+\mathbf{p} @ 2,2 \mathrm{GeV}: \phi$ - Efficiency Distributions

These histograms show the efficiency as a function of the $\phi$-angle eff $(\phi)$ as defined in Section 4.4.2 for several selected settings of the correlation algorithm. The first three distributions are flat, except for the edges of the chambers where the limited statistics prevents the results from being very meaningful. Starting from there, it is visible that with more aggressive trigger parameters the distribution starts to get bumps and the efficiency in sector 0 strongly drops. This is most likely connected to the hot spot in the same sector (compare Figure 4.5). 

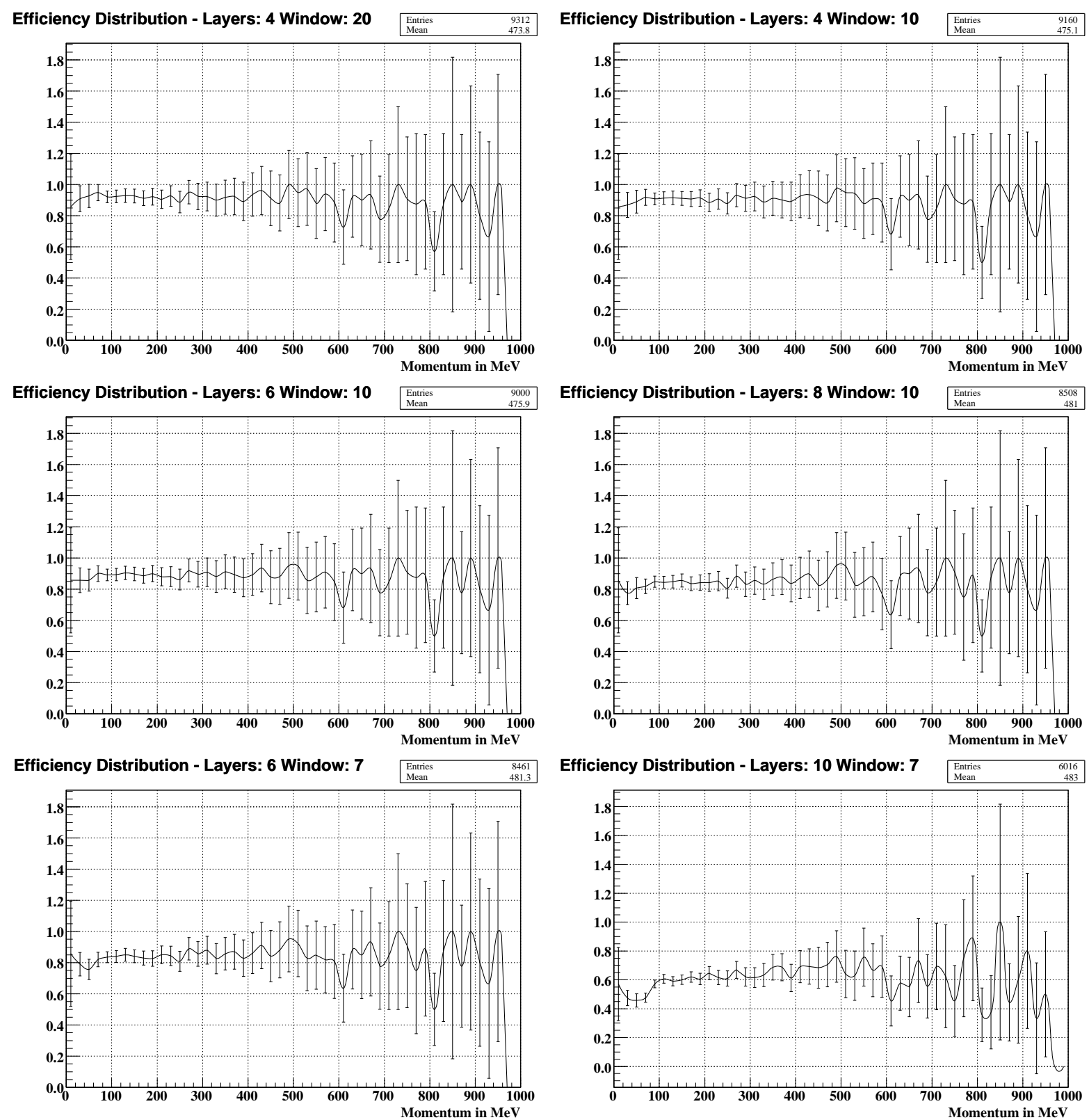

Figure 4.13.: $p+p @ 2,2 \mathrm{GeV}$ : Momentum Efficiency Distributions

These histograms show the efficiency as a function of the momentum eff $(p)$ as defined in Section 4.4.2 for several selected settings of the correlation algorithm. It is interesting to see that in spite of the effects in $\vartheta$ and $\phi$ for certain parameter settings the efficiency distribution remains essentially flat at these settings, at least up to a setting of 6 layers and 10 cells. The large error bars are caused by the very low statistics beyond $400 \mathrm{MeV}$ (compare Figure 4.10). 


\subsubsection{Correlation Analysis - $\mathrm{C}+\mathrm{C}$ at $1,0 \mathrm{AGeV}$}

All the presented figures in this section are derived from several consecutive experimental runs of the August 2004 beamtime. The target was a $6,5 \mathrm{~mm}$ thick carbon target and the beam energy was $1 \mathrm{AGeV}$. The generation $3 \mathrm{DST}$ macros and parameters have been used. For the deduction of the correlation parameters only the first run was used.
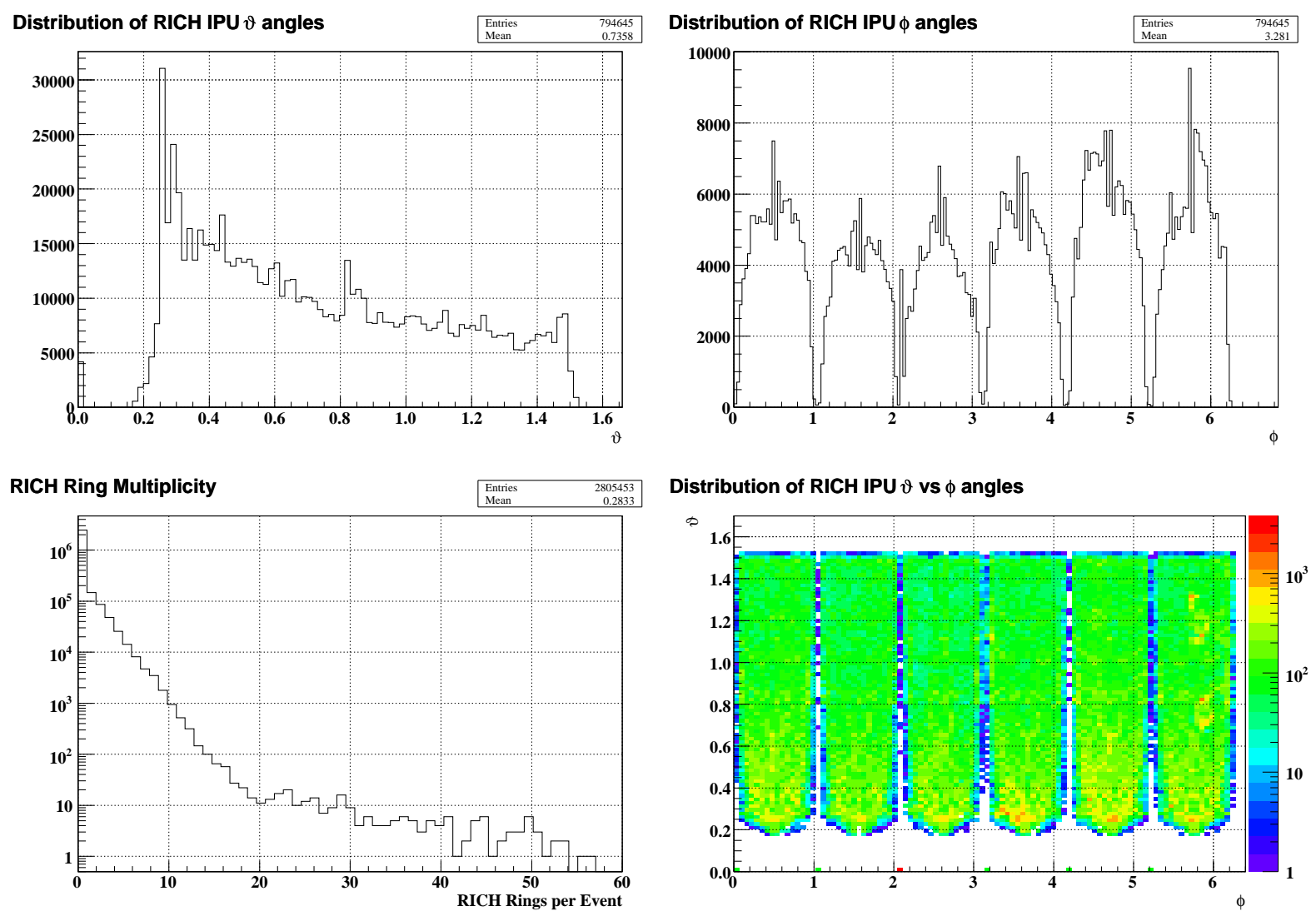

Figure 4.14.: C+C@1,0 AGeV: RICH Ring Distribution from the RICH IPU (available online).

The histograms show the distributions of the RICH rings as they are reconstructed by the RICH IPU (compare Figure 2.4). The bottom left histogram shows the RICH IPU ring multiplicity per event. The six different sectors are visible in the $\phi$-distribution and are numbered from left to right as 5, 0, 1, 2, 3, 4 (definition of coordinate system: Figure 2.1). The two dimensional histogram shows $\vartheta$ versus $\phi$ and shows if there is a very noisy region in the RICH, which is not the case for this data set. 

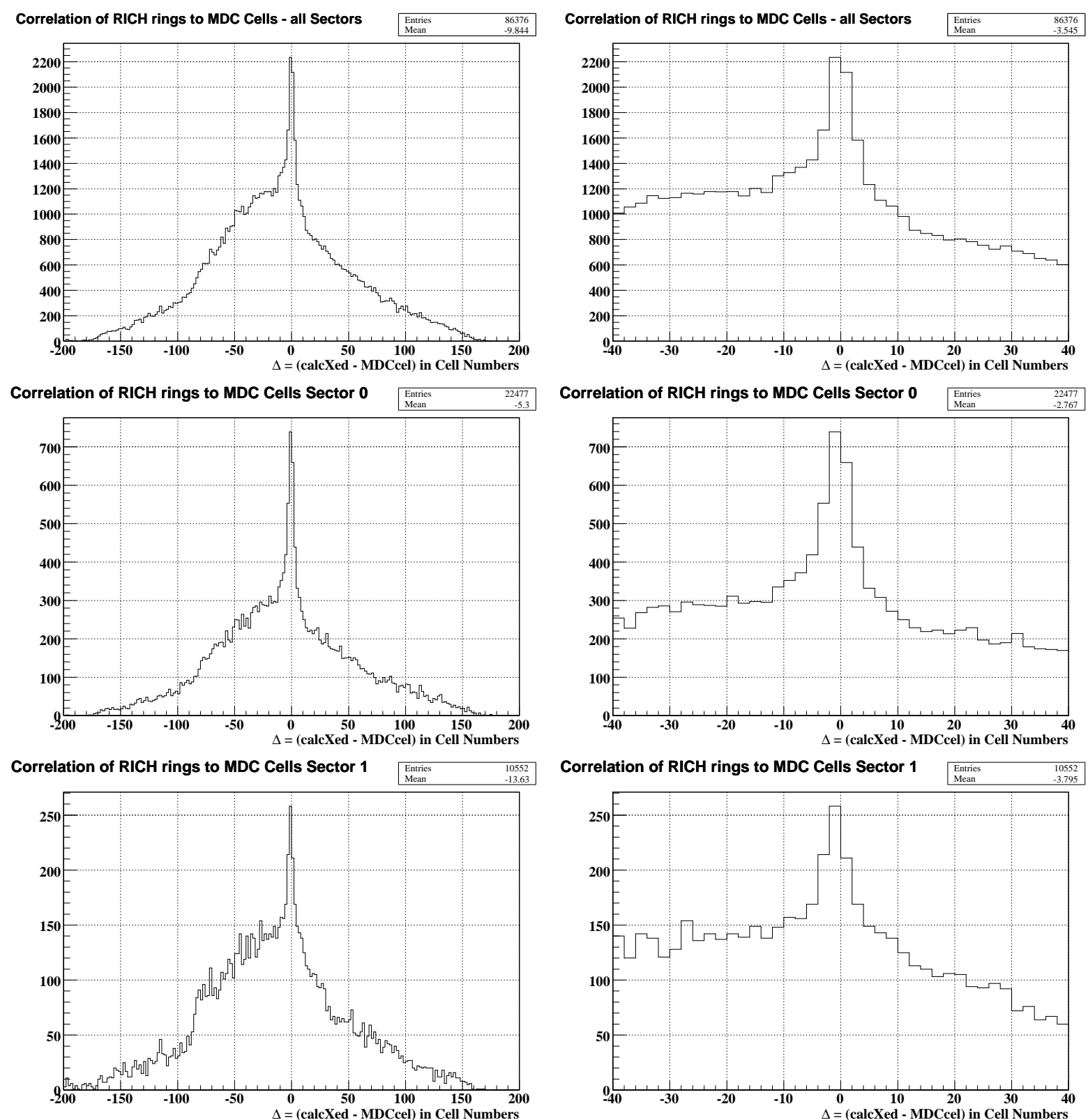

Figure 4.15.: C+C@1,0 AGeV: RICH IPU to MDC Cell Correlation first Run (available online).

The histograms on the right side show a magnified view of the correlation histograms on the left. The presence of a clear peak at 0 shows that the correlation is working, and the width of the correlation peak allows to decide which correlation window is safe to use. A safe window is 10 cells, while a more aggressive (but still realistic) cut is 7 cells or even 5 cells. The direct comparison with Figure 4.18 shows how these cuts affect the accepted good leptons. This histogram uses only the data from the first run to demonstrate the statistics needed to deduce the correlation parameters. The full statistics can be found in Figure 4.17. 

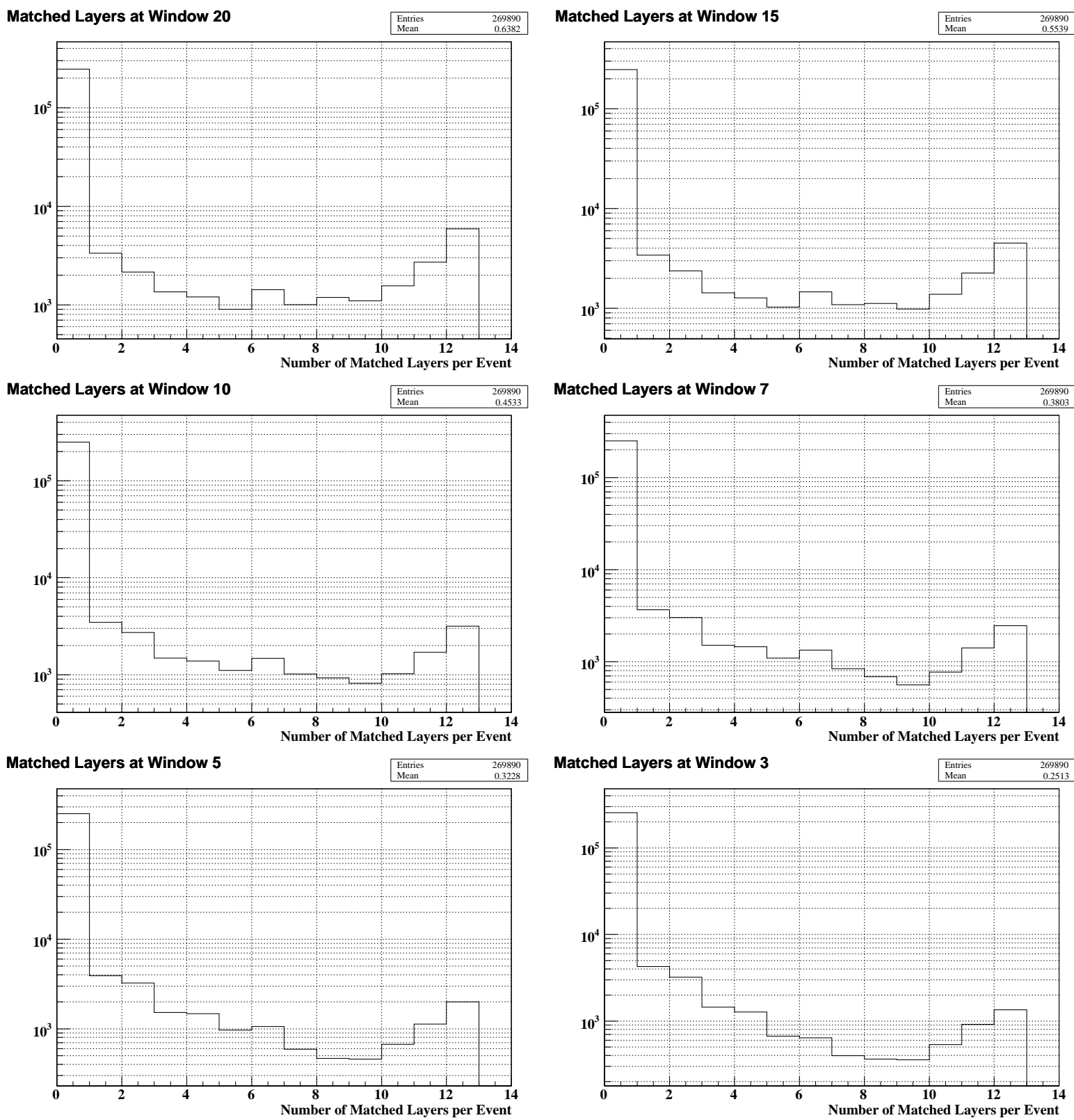

Figure 4.16.: C+C@1,0 AGeV: Matched Layers Distributions first Run (available online).

The histograms show how many layers can be matched for different settings of the width of the correlation window. Events with no correlation (or little/random) end up in the bins to the left. Interesting to note is the rise of the distributions at higher numbers of matched layers, as long as the matching window is still large enough (here in all cases). The save cut is at 4 layers (compare Section 4.2.1). Assuming that correlated leptons have a larger number of matched layers, one can look at the rise on the right and place the cut before it starts. This means for the just deduced window cuts of 10 cells, 7 cells and 5 cells a conservative layer cut is at 8 layers. A more aggressive cut is 10 layers. These cuts should be compared to the histograms in Figure 4.20 to check the effect of this layer cut onto the accepted good leptons. This histogram uses only the data from the first run to demonstrate the statistics needed to deduce the correlation parameters. The full statistics can be found in Figure 4.19. 

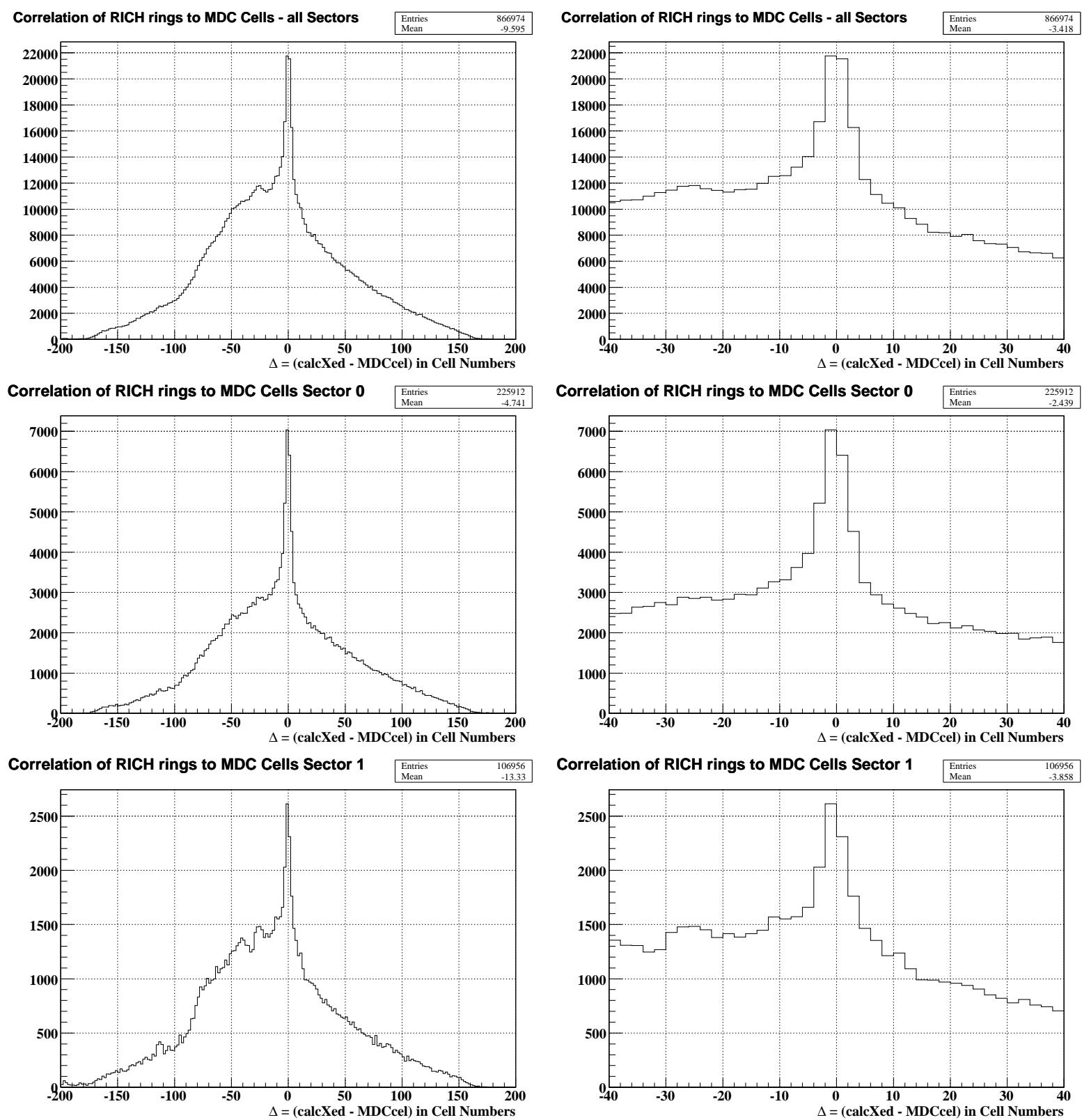

Figure 4.17.: $\mathrm{C}+\mathrm{C} @ 1,0 \mathrm{AGeV}$ : RICH IPU Ring to MDC Cell Correlation (available online).

The histograms on the right side show a magnified view of the correlation histograms on the left. The comparison of these histograms with Figure 4.15 shows that the deduced correlation parameters are also valid for a larger data set. 

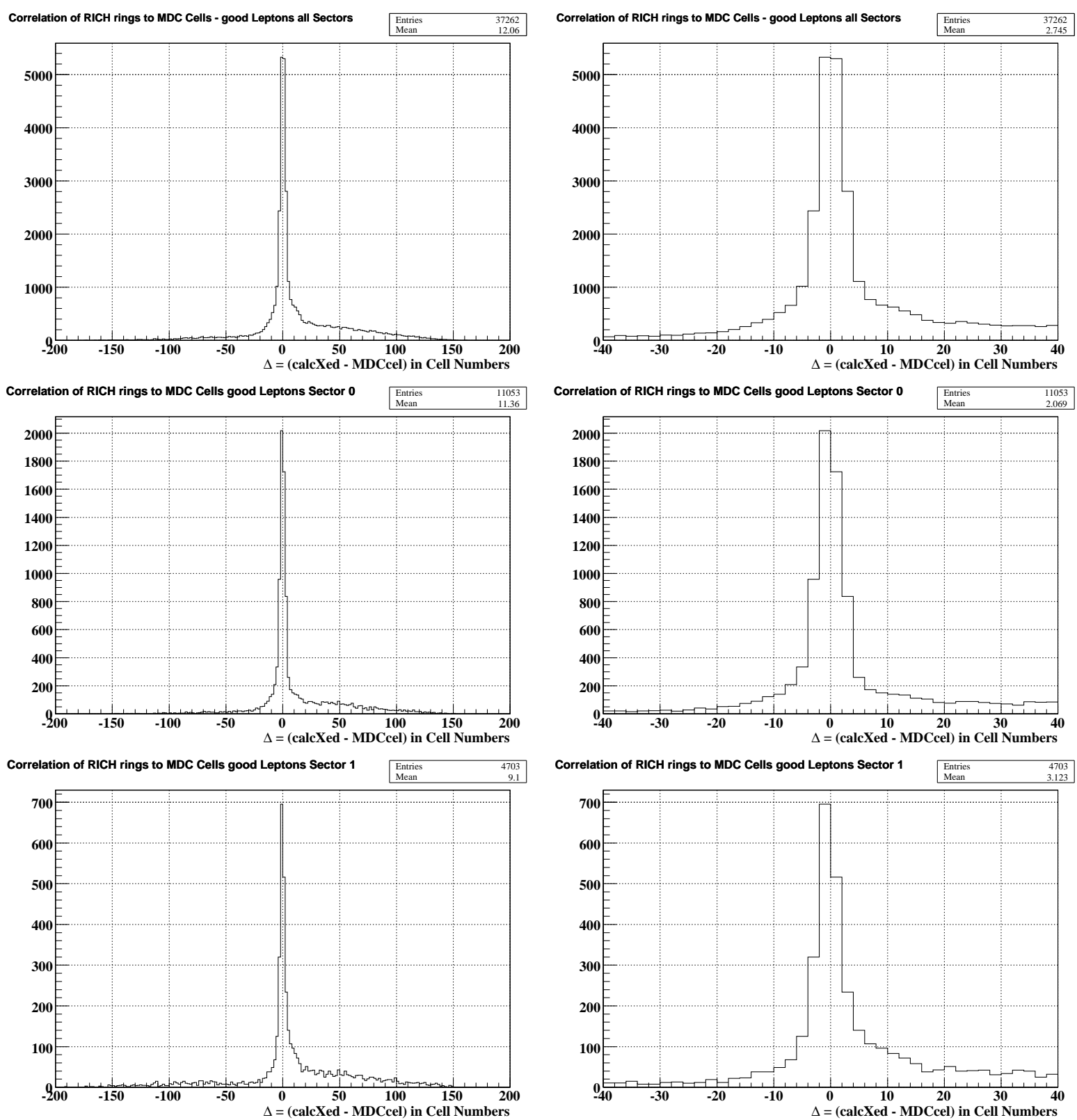

Figure 4.18.: C+C@1,0 AGeV: RICH IPU Ring to MDC Cell Correlation for good Leptons.

The histograms on the right side show a magnified view of the correlation histograms on the left. The presence of a clear peak at 0 shows that the correlation is working well for the good leptons and the width of the correlation peak shows how narrow the matching window cut could be in principle for the correlated good leptons to be accepted. Comparing the width of these histograms with the width deduced from Figure 4.17 it is clear, that a cut of 10 cells is really safe and a cut with a width of down to 5 cells is realistic. 

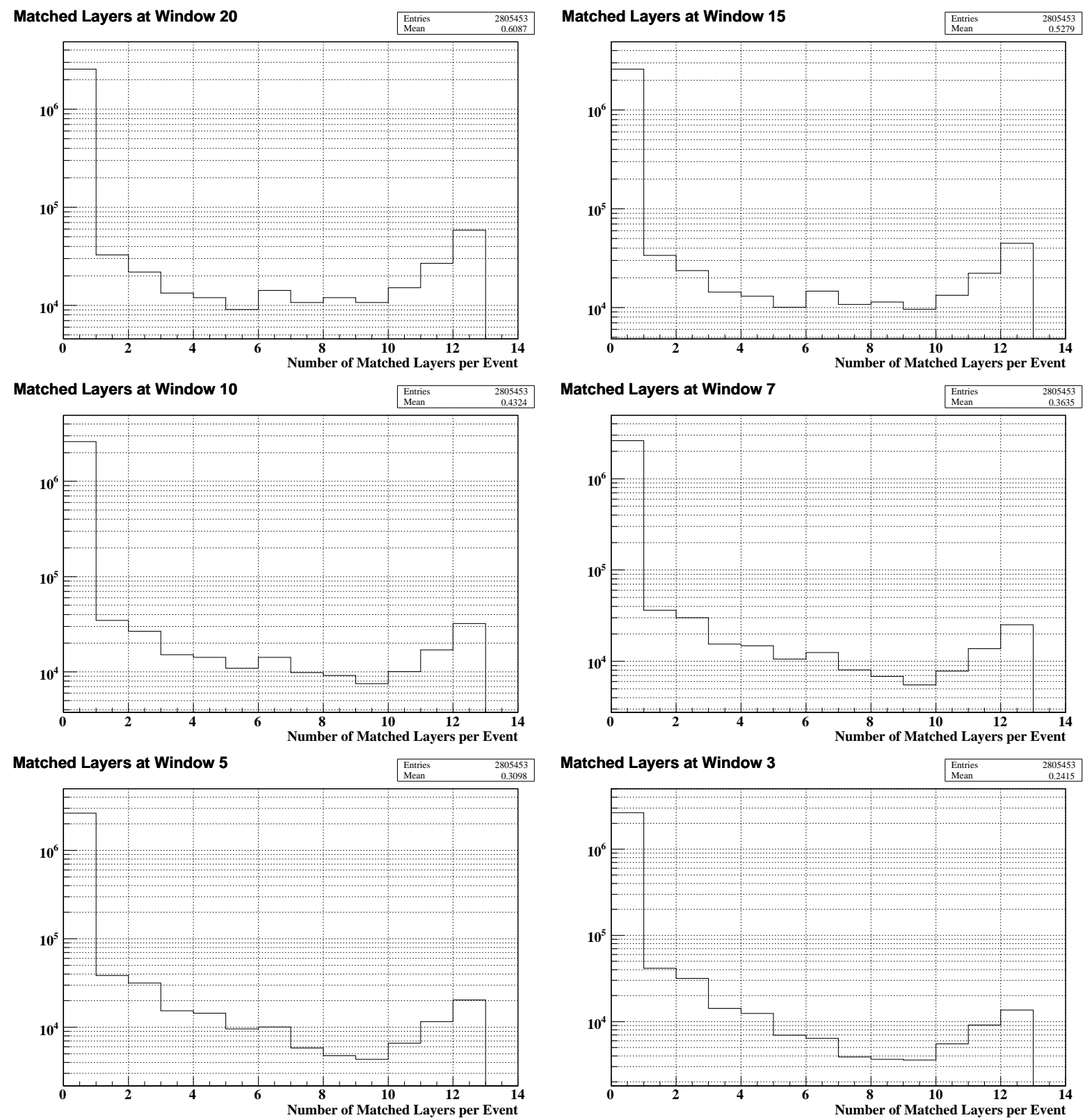

Figure 4.19.: C+C@1,0 AGeV: Matched Layers Distributions (available online).

The histograms show how many layers can be matched for different settings of the width of the correlation window. Events with no correlation (or little/random) end up in the bins to the left. Interesting to note is the rise of the distributions at higher numbers of matched layers, as long as the matching window is still large enough (here in all cases).

The comparison of these histograms with Figure 4.16 shows that the deduced correlation parameters are also valid for a larger data set. 

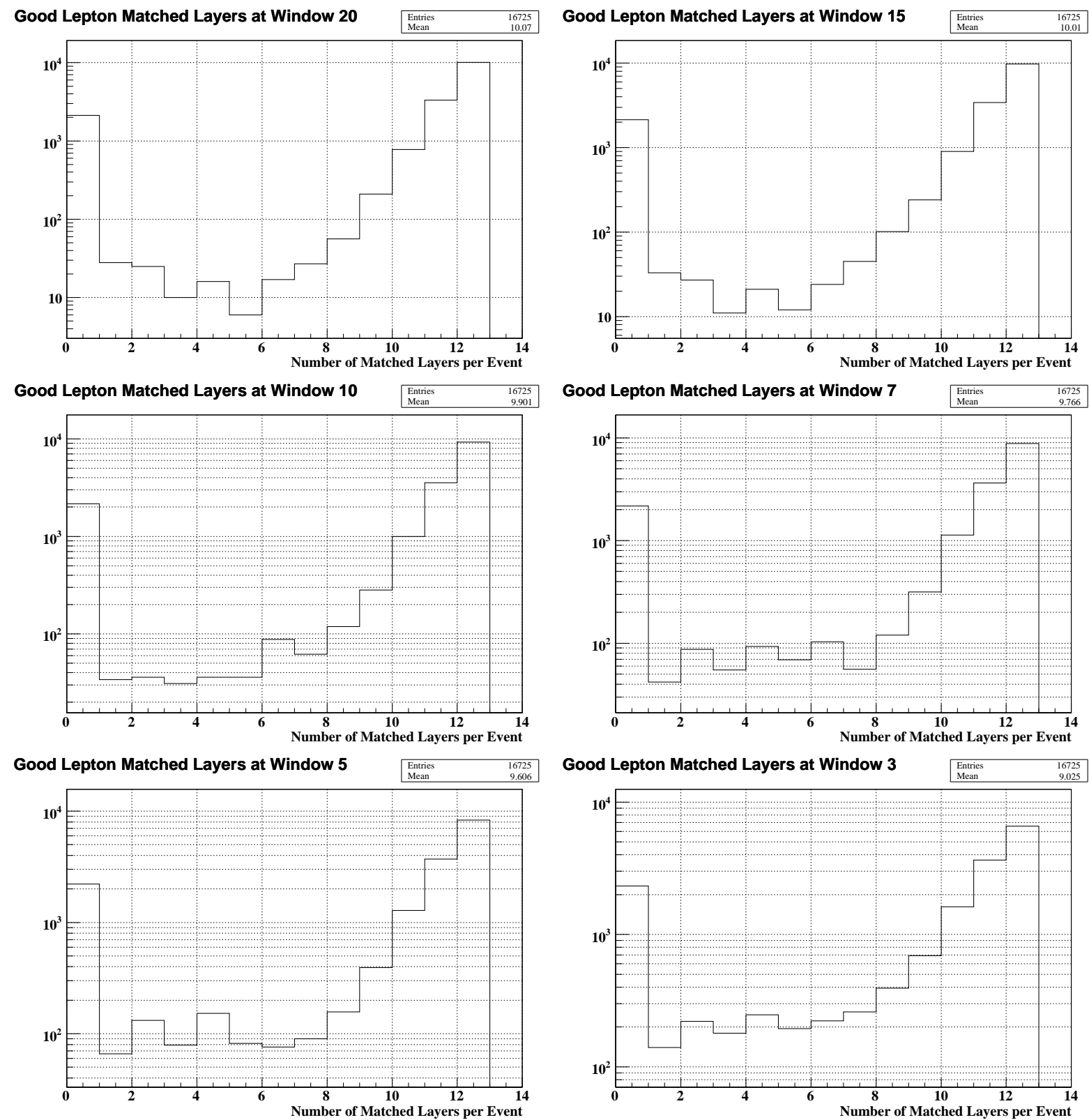

Figure 4.20:: C+C@1,0 AGeV: Matched Layers Distributions for good Lepton Events.

The Figures show the number of matched layers distributions for different settings of the matching window. These histograms show only events with good leptons. The strong rise towards a higher number of matched layers for these good events is clearly visible, as well as the valley around 4 matched layers for moderate window sizes (20 to 10). It should be noted that the bin at zero matched layers is fairly constant for different settings. Comparing these histograms with the just deduced minimum number of layers cuts it is clearly visible, that a cut at 8 layers is always before the rise and a cut at 10 layers starts to cut just into the rise of the correlated good leptons. 


\begin{tabular}{|c|c|c|c|c|c|c|c|c|c|c|}
\hline \multicolumn{4}{|c|}{ Trigger Analysis for run: } & \multicolumn{4}{|c|}{ August 2004} & \multicolumn{3}{|c|}{ combined } \\
\hline Result & L2 & L3 & M & Layers & Window & red & eff & eff 2 & enh & enh2 \\
\hline 0 & Yes & No & 1 & 4 & 20 & 0,054 & 0,743 & n.a. & 13,680 & n.a. \\
\hline 1 & No & Yes & 1 & 4 & 20 & 0,060 & 0,869 & 0,989 & 14,445 & 16,423 \\
\hline 2 & No & Yes & 1 & 4 & 15 & 0,053 & 0,868 & 0,987 & 16,274 & 18,502 \\
\hline 3 & No & Yes & 1 & 4 & 10 & 0,045 & 0,865 & 0,984 & 19,408 & 22,065 \\
\hline 4 & No & Yes & 1 & 4 & 7 & 0,037 & 0,859 & 0,977 & 22,986 & 26,133 \\
\hline 5 & No & Yes & 1 & 4 & 5 & 0,031 & 0,851 & 0,968 & 27,292 & 31,028 \\
\hline 6 & No & Yes & 1 & 6 & 20 & 0,053 & 0,868 & 0,987 & 16,478 & 18,733 \\
\hline 7 & No & Yes & 1 & 6 & 15 & 0,045 & 0,866 & 0,985 & 19,186 & 21,813 \\
\hline 8 & No & Yes & 1 & 6 & 10 & 0,036 & 0,861 & 0,979 & 24,168 & 27,477 \\
\hline 9 & No & Yes & 1 & 6 & 7 & 0,028 & 0,849 & 0,966 & 29,985 & 34,090 \\
\hline 10 & No & Yes & 1 & 6 & 5 & 0,023 & 0,837 & 0,952 & 37,006 & 42,072 \\
\hline 11 & No & Yes & 1 & 8 & 20 & 0,044 & 0,866 & 0,984 & 19,755 & 22,460 \\
\hline 12 & No & Yes & 1 & 8 & 15 & 0,036 & 0,862 & 0,980 & 23,884 & 27,154 \\
\hline 13 & No & Yes & 1 & 8 & 10 & 0,027 & 0,852 & 0,969 & 31,514 & 35,829 \\
\hline 14 & No & Yes & 1 & 8 & 7 & 0,021 & 0,840 & 0,955 & 39,974 & 45,446 \\
\hline 15 & No & Yes & 1 & 8 & 5 & 0,017 & 0,827 & 0,941 & 48,760 & 55,435 \\
\hline 16 & No & Yes & 1 & 10 & 20 & 0,036 & 0,850 & 0,966 & 23,784 & 27,040 \\
\hline 17 & No & Yes & 1 & 10 & 15 & 0,029 & 0,842 & 0,957 & 29,396 & 33,420 \\
\hline 18 & No & Yes & 1 & 10 & 10 & 0,021 & 0,828 & 0,941 & 39,252 & 44,626 \\
\hline 19 & No & Yes & 1 & 10 & 7 & 0,017 & 0,814 & 0,925 & 48,956 & 55,658 \\
\hline 20 & No & Yes & 1 & 10 & 5 & 0,014 & 0,795 & 0,903 & 57,860 & 65,781 \\
\hline 21 & No & Yes & 1 & 10 & 3 & 0,010 & 0,709 & 0,806 & 70,428 & 80,070 \\
\hline 22 & Yes & Yes & 1 & 4 & 20 & 0,035 & 0,740 & 0,842 & 20,999 & 23,874 \\
\hline 23 & Yes & Yes & 1 & 4 & 15 & 0,032 & 0,740 & 0,841 & 23,236 & 26,417 \\
\hline 24 & Yes & Yes & 1 & 4 & 10 & 0,027 & 0,738 & 0,839 & 27,005 & 30,702 \\
\hline 25 & Yes & Yes & 1 & 4 & 7 & 0,023 & 0,733 & 0,833 & 31,257 & 35,537 \\
\hline 26 & Yes & Yes & 1 & 4 & 5 & 0,020 & 0,727 & 0,827 & 36,409 & 41,393 \\
\hline 27 & Yes & Yes & 1 & 6 & 20 & 0,032 & 0,740 & 0,841 & 23,318 & 26,510 \\
\hline 28 & Yes & Yes & 1 & 6 & 15 & 0,028 & 0,738 & 0,839 & 26,620 & 30,264 \\
\hline 29 & Yes & Yes & 1 & 6 & 10 & 0,023 & 0,735 & 0,835 & 32,628 & 37,095 \\
\hline 30 & Yes & Yes & 1 & 6 & 7 & 0,018 & 0,726 & 0,826 & 39,648 & 45,076 \\
\hline 31 & Yes & Yes & 1 & 6 & 5 & 0,015 & 0,717 & 0,815 & 47,878 & 54,433 \\
\hline 32 & Yes & Yes & 1 & 8 & 20 & 0,027 & 0,738 & 0,839 & 27,117 & 30,830 \\
\hline 33 & Yes & Yes & 1 & 8 & 15 & 0,023 & 0,736 & 0,837 & 32,149 & 36,550 \\
\hline 34 & Yes & Yes & 1 & 8 & 10 & 0,018 & 0,729 & 0,829 & 41,549 & 47,238 \\
\hline 35 & Yes & Yes & 1 & 8 & 7 & 0,014 & 0,720 & 0,819 & 51,472 & 58,518 \\
\hline 36 & Yes & Yes & 1 & 8 & 5 & 0,012 & 0,709 & 0,807 & 61,368 & 69,769 \\
\hline 37 & Yes & Yes & 1 & 10 & 20 & 0,023 & 0,725 & 0,824 & 31,925 & 36,296 \\
\hline 38 & Yes & Yes & 1 & 10 & 15 & 0,019 & 0,720 & 0,819 & 38,889 & 44,213 \\
\hline 39 & Yes & Yes & 1 & 10 & 10 & 0,014 & 0,710 & 0,807 & 50,678 & 57,616 \\
\hline 40 & Yes & Yes & 1 & 10 & 7 & 0,011 & 0,698 & 0,793 & 61,649 & 70,089 \\
\hline 41 & Yes & Yes & 1 & 10 & 5 & 0,010 & 0,681 & 0,775 & 71,001 & 80,721 \\
\hline 42 & Yes & Yes & 1 & 10 & 3 & 0,007 & 0,608 & 0,691 & 82,996 & 94,359 \\
\hline 43 & No & Yes & 2 & 4 & 20 & 0,028 & 0,497 & 0,534 & 17,450 & 18,760 \\
\hline 44 & No & Yes & 2 & 4 & 10 & 0,018 & 0,489 & 0,525 & 26,439 & 28,424 \\
\hline 45 & No & Yes & 2 & 8 & 7 & 0,008 & 0,433 & 0,466 & 56,310 & 60,536 \\
\hline 46 & No & Yes & 2 & 8 & 10 & 0,010 & 0,458 & 0,492 & 43,828 & 47,117 \\
\hline 47 & No & Yes & 2 & 10 & 5 & 0,004 & 0,339 & 0,364 & 87,339 & 93,895 \\
\hline 48 & No & Yes & 2 & 10 & 3 & 0,002 & 0,209 & 0,225 & 116,933 & 125,710 \\
\hline
\end{tabular}

Table 4.4.: C+C@1,0 AGeV: Efficiency and Enhancement for different Correlation Parameters.

This table shows a systematic calculation of efficiency and enhancement for different settings of the correlation parameters. The L3 column denotes if the RICH to MDC correlation trigger was active, while L2 denotes the same for the HADES level 2 trigger system. Multiplicity (M) is the minimum number of required good leptons per event. Result 0 shows the results of the HADES level 2 trigger for the same data set for comparison. From this table and the discussion of the previous distributions a particular useful trigger setting would be for example 8 layers and 7 cells (Result: 14). This conservative parameter set gives a reasonable enhancement while still having a good efficiency. It should be noted that this is still true for a more aggressive setting of 10 layers and 5 cells (Result: 20). Only with even more aggressive settings the efficiency starts to drop fast (Result: 21). These parameter sets should be cross referenced with the efficiency distributions in Figure 4.22, Figure 4.23 and Figure 4.24. The values of eff2 and enh2 demonstrate the influence of the RICH IPU (compare Section 4.4.4). 112 

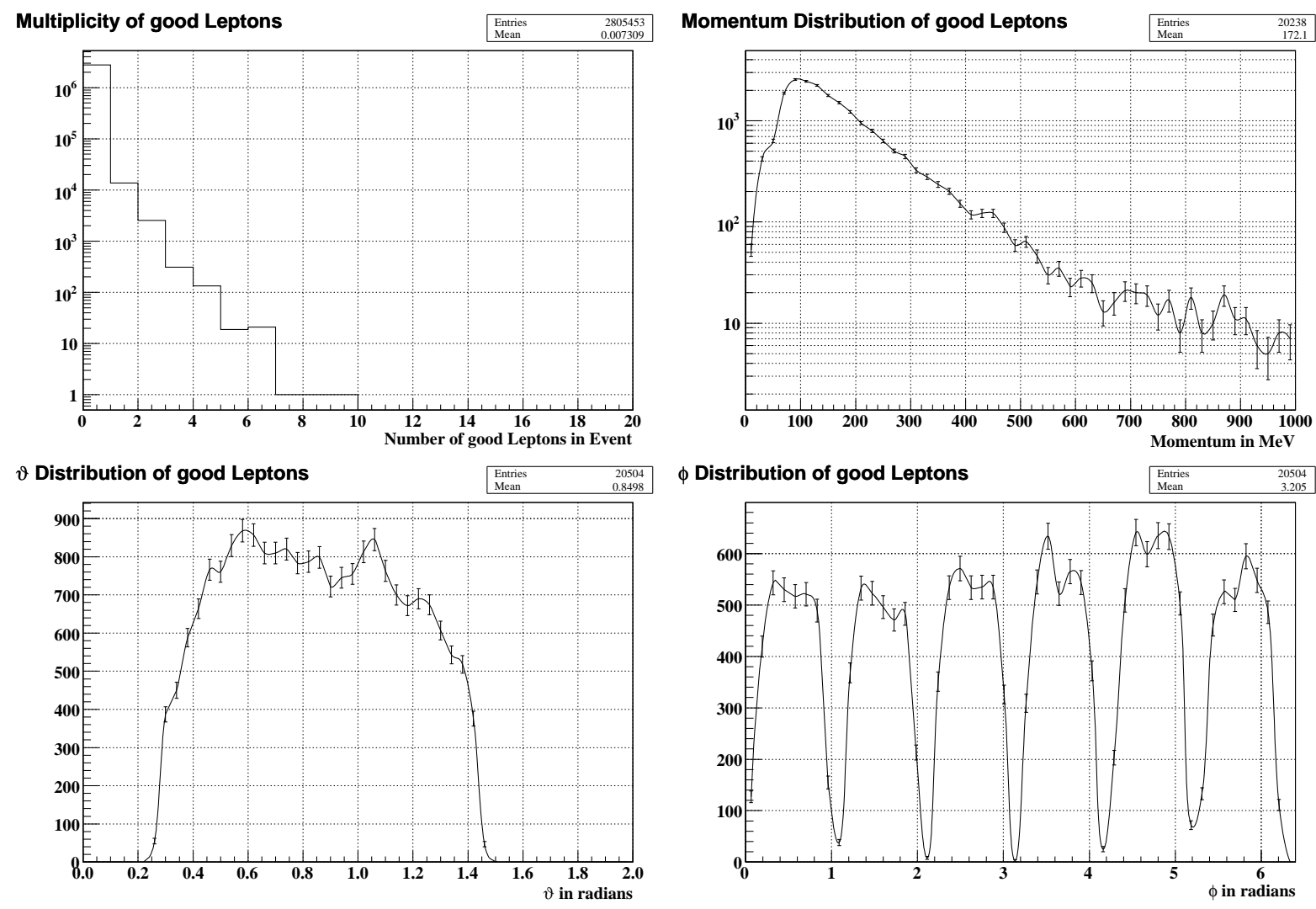

Figure 4.21.: C+C@1,0 AGeV: Good Lepton Base Data.

The histograms show the base data of the good leptons. Their multiplicity per event histogram shows how few events actually contain one or more good lepton. The $\vartheta$-distribution and the $\phi$ distribution show the respective angles of the lepton before the magnetic field and clearly follow the geometrical structure of the HADES detector. The momentum distribution is obtained from the "spline" algorithm, using the bending of the track in the magnetic field. These distributions provide the good total events in Equation (4.10). Dividing the accepted good lepton distributions for different correlation parameters with these histograms gives the different efficiency distribution histograms. It should be noted that the statistics are not very large for momenta above $500 \mathrm{MeV}$. The errors are deduced from the statistical errors only. 

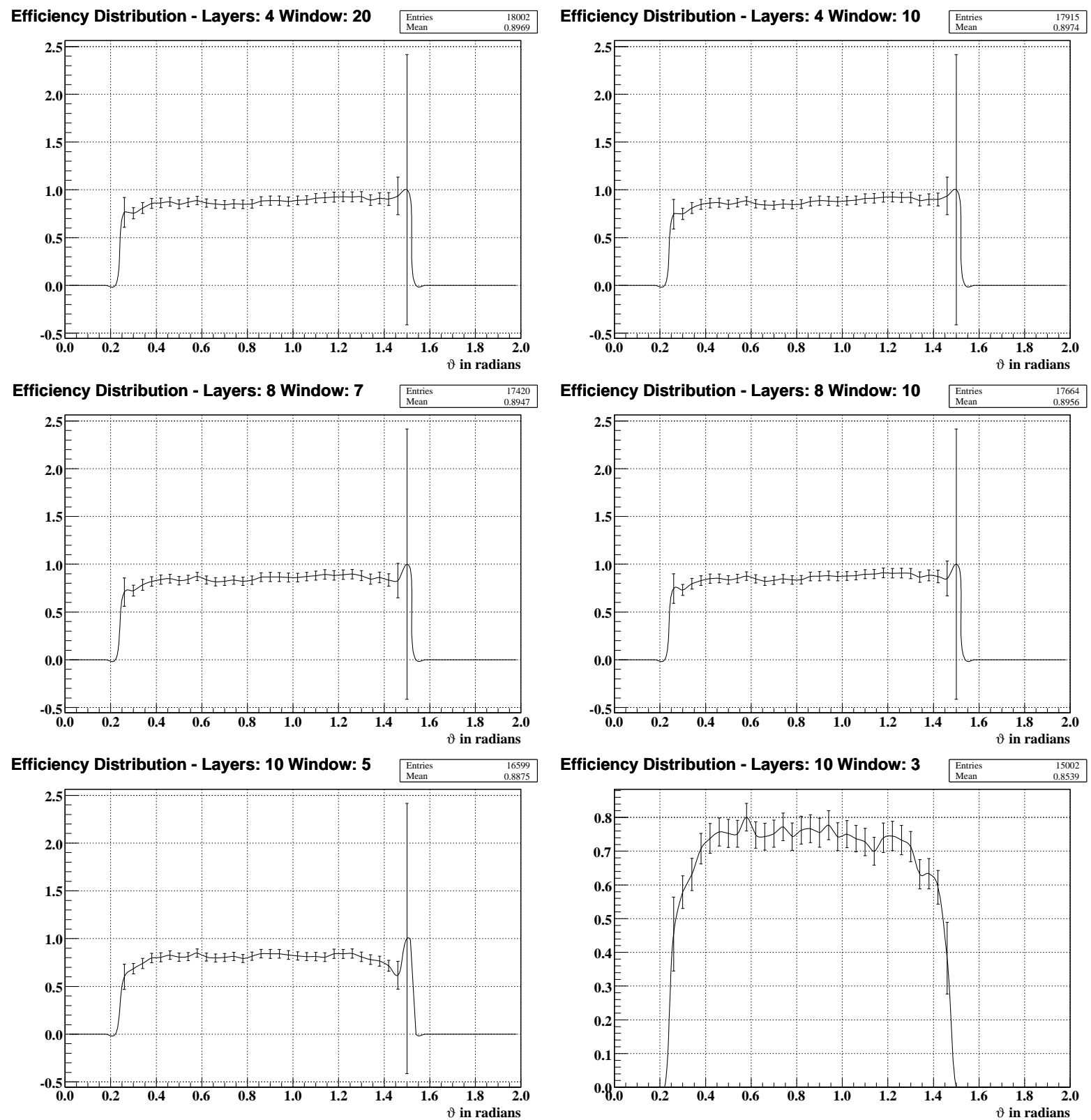

Figure 4.22.: C+C@1,0 AGeV: $\vartheta$ - Efficiency Distributions

These histograms show the efficiency as a function of the $\vartheta$-angle eff $(\vartheta)$ as defined in Section 4.4.2 for several selected settings of the correlation algorithm. For up to the deduced trigger settings, the distributions are flat, except for the edges of the chambers where the limited statistics prevents the results from being very conclusive. Only with the very aggressive setting of 10 layers and 3 cells the efficiency becomes significantly distorted. 

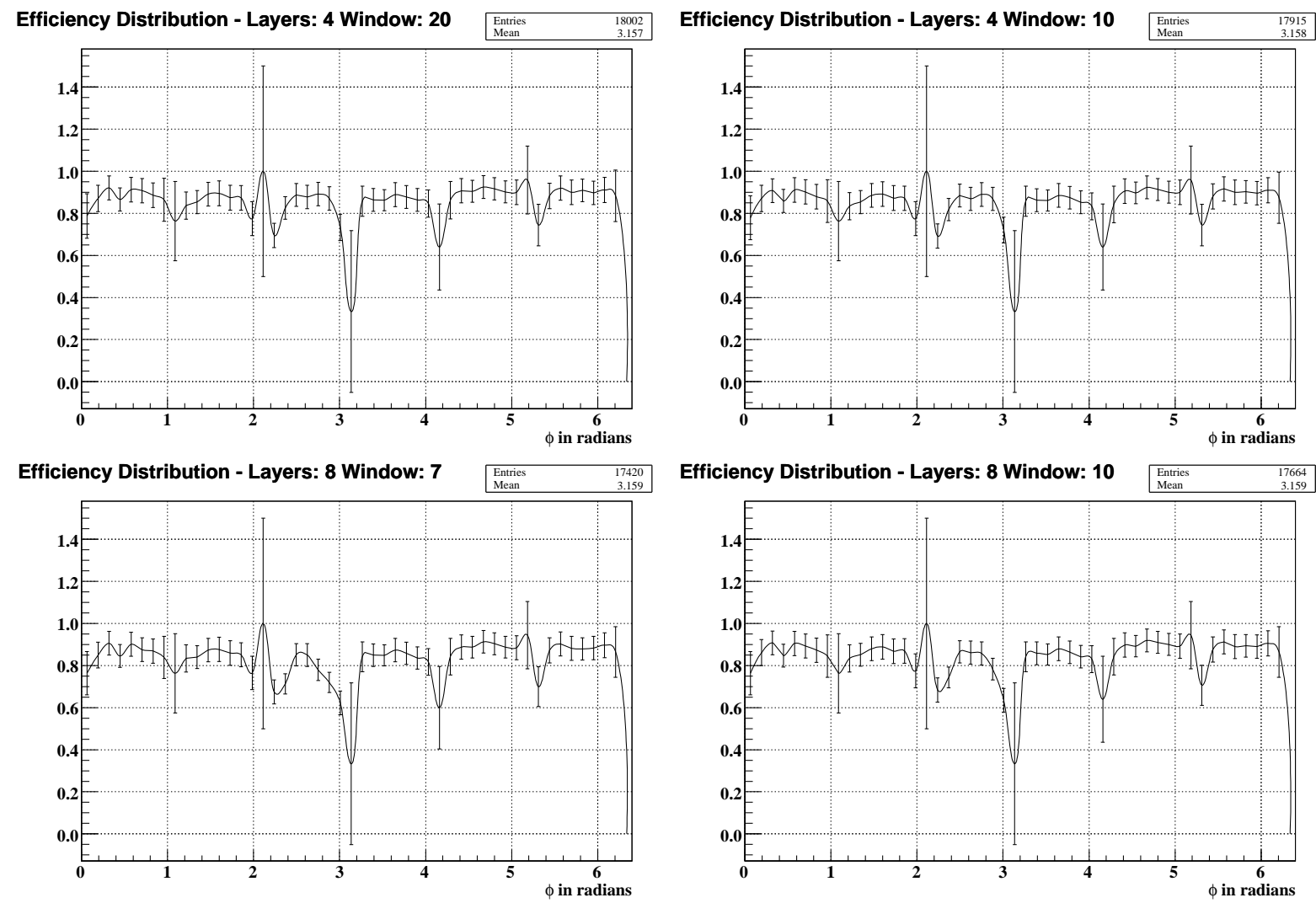

Efficiency Distribution - Layers: 8 Window: 10
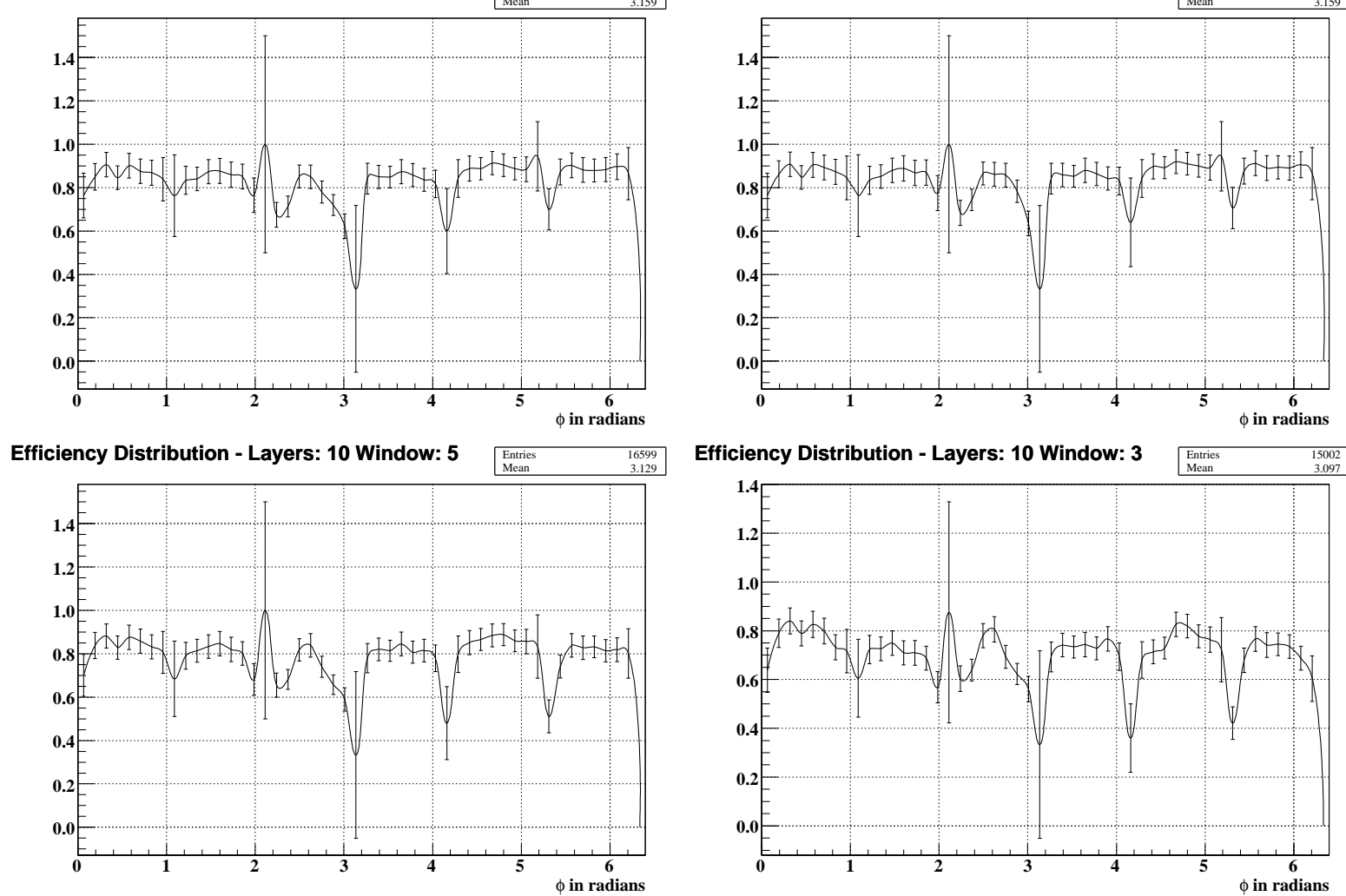

Figure 4.23.: C+CO1,0 AGeV: $\phi$ - Efficiency Distributions

These histograms show the efficiency as a function of the $\phi$-angle eff $(\phi)$ as defined in Section 4.4.2 for several selected settings of the correlation algorithm. For up to the deduced trigger settings, the distributions are flat, except for the edges of the chambers where the limited statistics prevents the results from being very conclusive. The exception to this is sector 1 , which shows already at the safe settings some distortion which becoming more and more apparent at stronger trigger settings. 

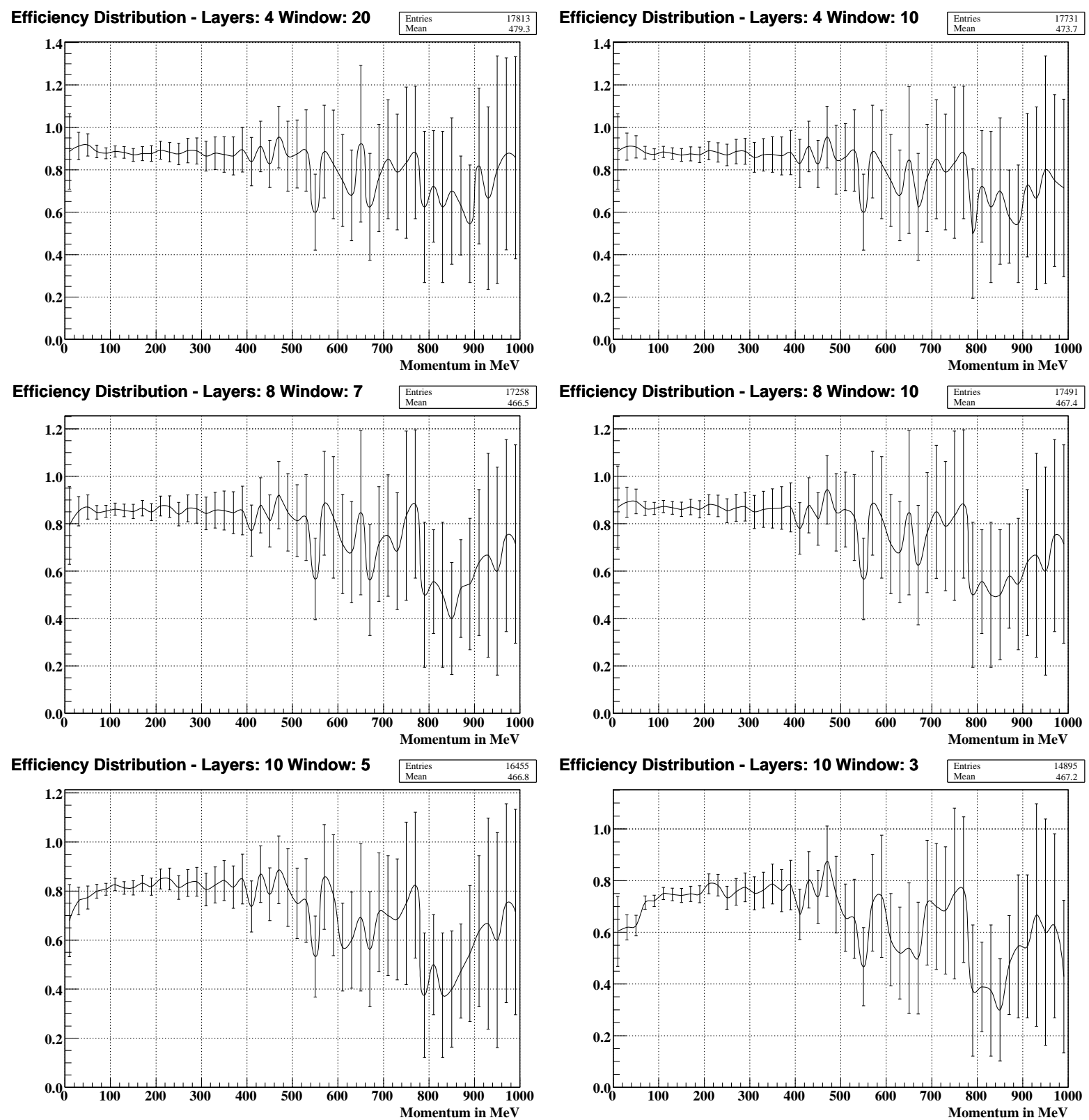

Figure 4.24.: C+C@1,0 AGeV: Momentum Efficiency Distributions

These histograms show the efficiency as a function of the momentum eff $(p)$ as defined in Section 4.4.2 for several selected settings of the correlation algorithm. The distributions are flat up to a momentum of $\approx 500 \mathrm{MeV}$ (for settings up to 10 layers and 5 cells). Beyond that, the limited statistics prevent a meaningful conclusion as indicated by the error bars (compare Figure 4.21). 


\subsubsection{Correlation Analysis - $\mathrm{Ar}+\mathrm{KCl}$ at $1,756 \mathrm{AGeV}$}

The presented figures in this section are derived from the date of several experimental consecutive runs of the September 2005 beamtime to increase statistics. The target was the 4 segmented Kalium-Chloride target (thickness 1,5 mm; z-Position: $z_{1}=-13,5 \mathrm{~mm}, z_{1}=-4,5 \mathrm{~mm}, z_{1}=$ $4,5 \mathrm{~mm}, z_{1}=13,5 \mathrm{~mm}$ ) and the beam energy was $1,756 \mathrm{AGeV}$. The generation $2 \mathrm{DST}$ macros and parameters are used. The increased statistics is crucial in Figure 4.26 to see the correlation peak and to be able to determine the matching window.
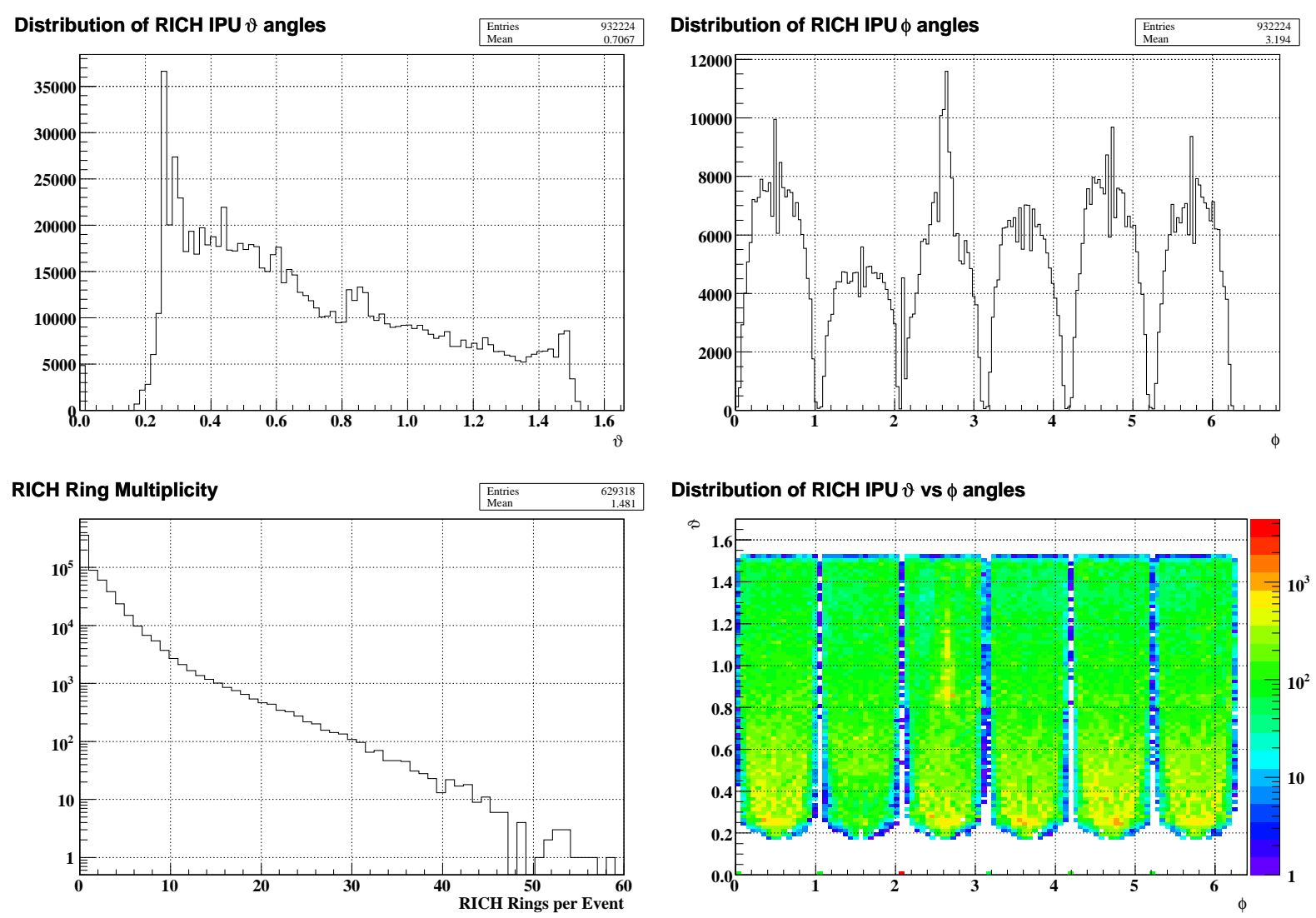

Figure 4.25.: Ar+KCI@1,756 AGeV: RICH Ring Distribution from RICH IPU (available online).

The histograms show the distributions of the RICH rings as they are reconstructed by the RICH IPU (compare Figure 2.4). The bottom left histogram shows the RICH IPU ring multiplicity per event. The bottom right histograms shows the $\phi$-distribution and $\vartheta$-distribution of these rings. The six different sectors are visible in the $\phi$-distribution and are numbered from left to right as $5,0,1,2,3,4$ (definition of coordinate system: Figure 2.1). The two dimensional histogram shows $\vartheta$ versus $\phi$ and shows if there is a very noisy region in the RICH, which is the case for the middle of sector 1 in this data set. 

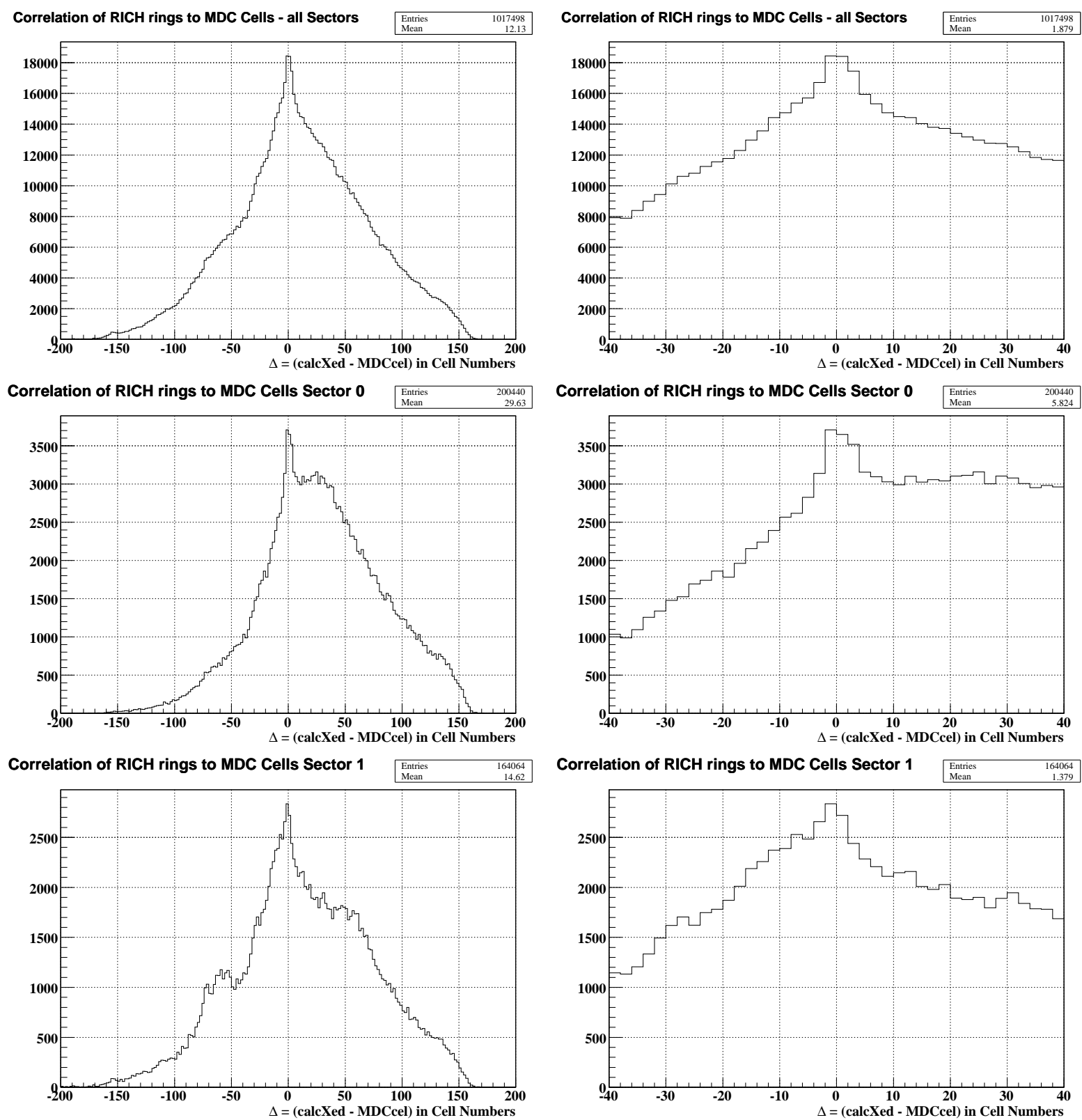

Figure 4.26.: Ar+KCI@1,756 AGeV: RICH IPU Ring to MDC Cell Correlation (available online).

The histograms on the right show a magnified view of the correlation histograms on the left. The statistics is just big enough to enhance the correlation peak at zero to enable the deduction of the matching window of 7 cells. The correlation peak vanishes with fewer statistics. These histograms should be compared with Figure 4.27. 

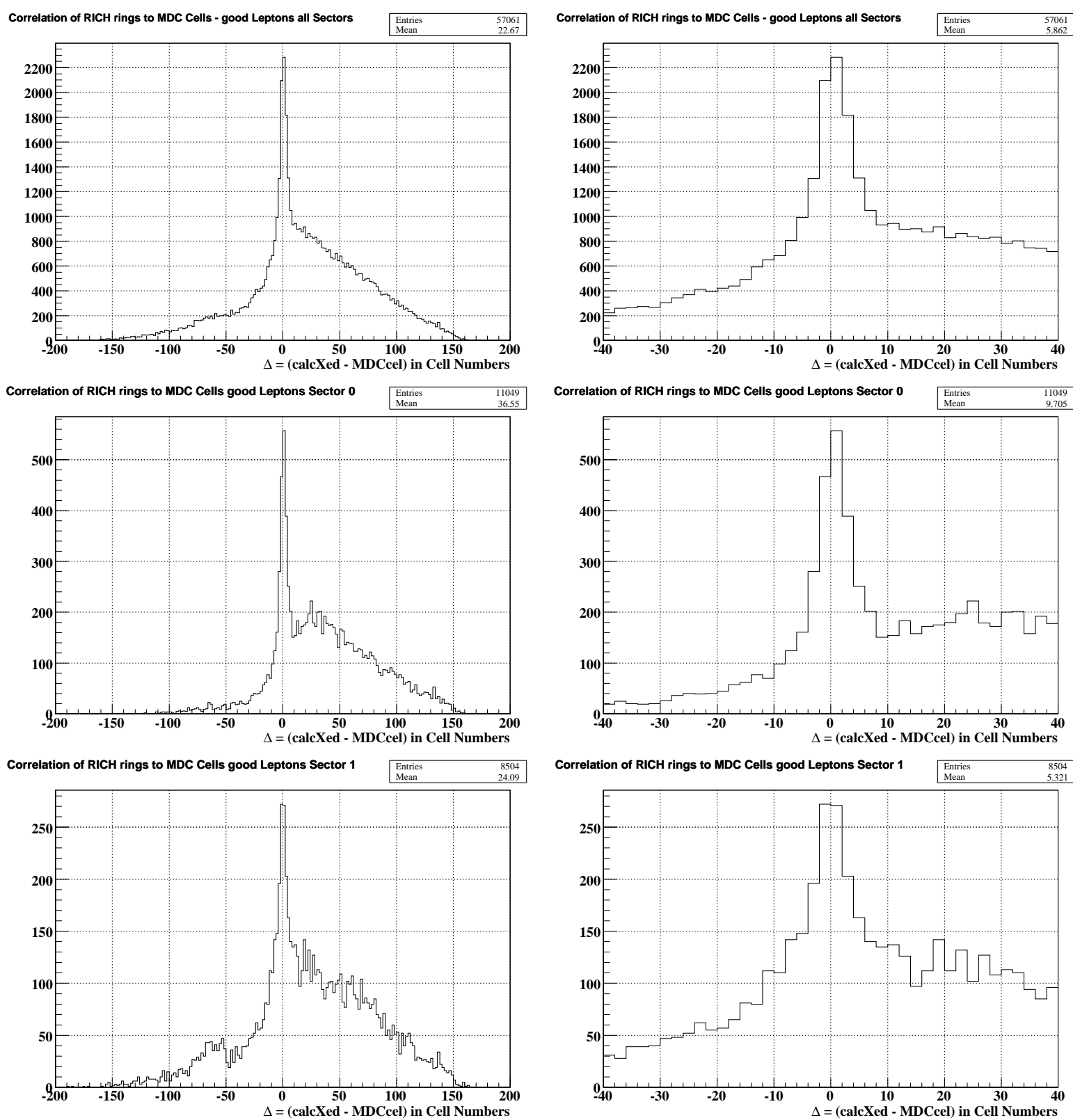

Figure 4.27.: Ar+KCI@1,756 AGeV: RICH IPU Ring to MDC Cell Correlation good Leptons.

The histograms on the right side show a magnified view of the correlation histograms on the left. The good lepton data shows a clear sharp peck at zero which is compatible with the matching window deduced from Figure 4.26. But the histograms also show much background, which might rise the question if the good lepton definition is perfect for this data. 

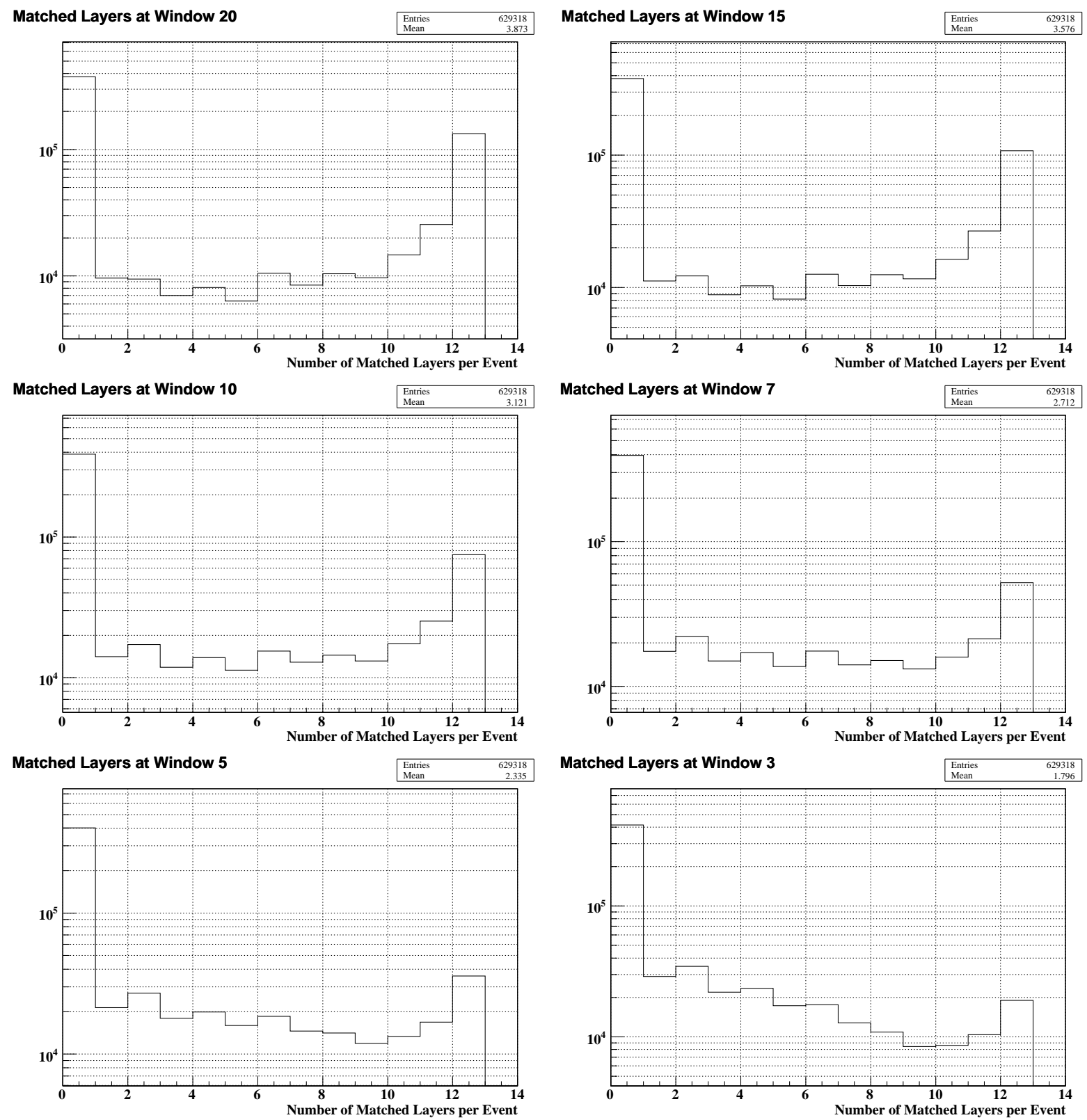

Figure 4.28.: Ar+KCl@1,756 AGeV: Matched Layers Distributions (available online).

The histograms show how many layers can be matched for different settings of the width of the correlation window. Events with no correlation (or little/random) end up in the bins to the left. Interesting to note is the strong rise of the distributions at higher numbers of matched layers $(10-12)$, as long as the matching window is still large enough $(\geqq 7)$. The save cut is at 4 layers (compare Section 4.2.1). Assuming that correlated leptons have a larger number of matched layers, one can look at the rise on the right and place the cut before it starts. This means for the assumed window a cut at 10 cells. This cut should be compared to the histograms in Figure 4.29 to check the effect of this layer cut onto the accepted good leptons. 

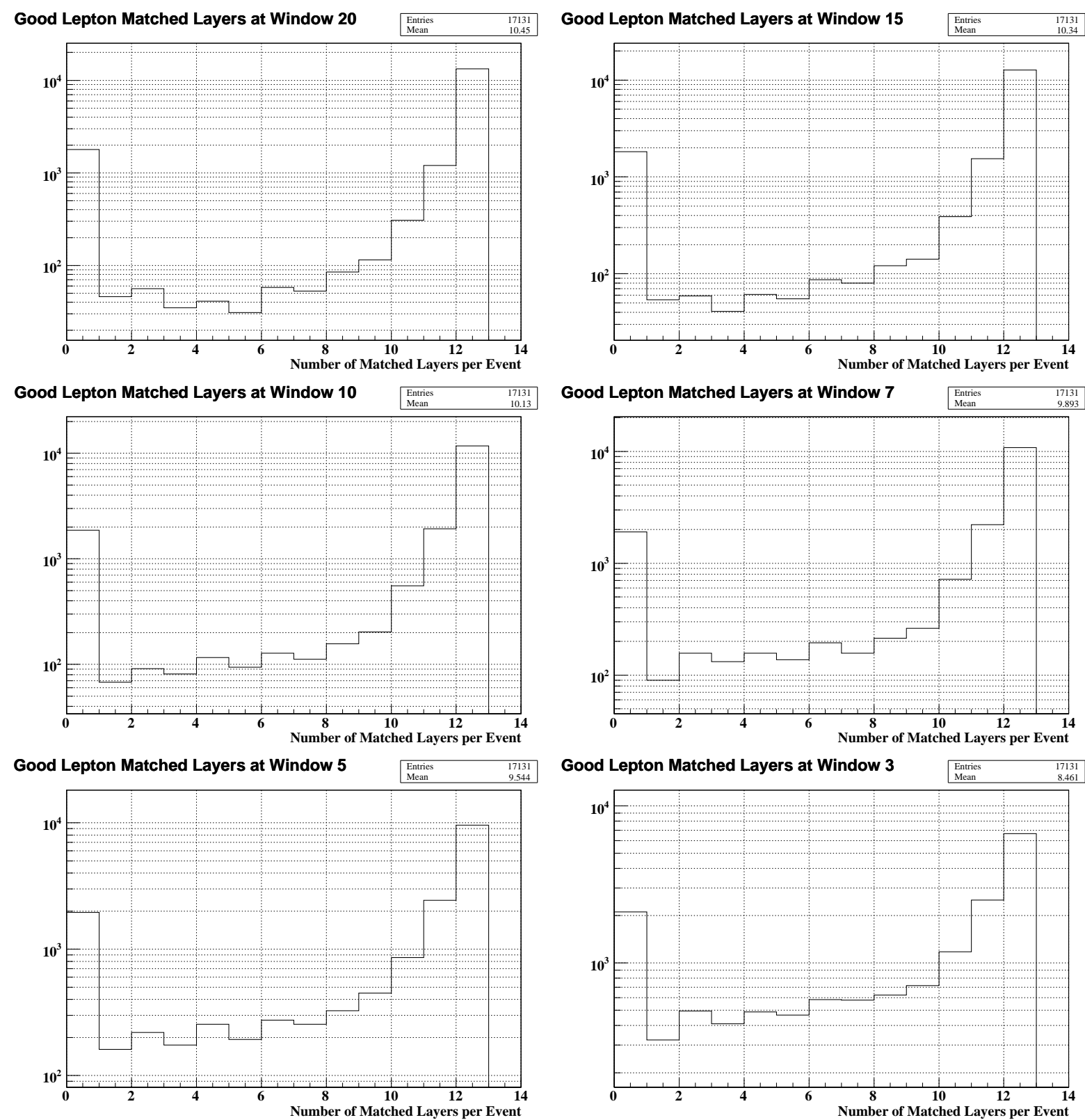

Figure 4.29.: Ar+KCl@1,756 AGeV: Matched Layers Distributions for good Lepton Events.

The Figures show the number of matched layers distributions for different settings of the matching window. These histograms show only events with good leptons. The strong rise towards a higher number of matched layers for these good events is clearly visible. Comparing these histograms with the just deduced minimum number of layers cuts, it is visible that a cut at 8 layers is always before the strong rise and a cut at 10 layers starts to cut just into the rise of the correlated good leptons. 


\begin{tabular}{|c|c|c|c|c|c|c|c|c|c|c|}
\hline \multicolumn{4}{|c|}{ Trigger Analysis for run: } & \multicolumn{4}{|c|}{ September 2005} & \multicolumn{3}{|c|}{ combined } \\
\hline Result & L2 & $\mathbf{L 3}$ & M & Layers & Window & red & eff & eff2 & enh & enh2 \\
\hline 0 & Yes & No & 1 & 4 & 20 & 0,252 & 0,795 & n.a. & 3,153 & n.a. \\
\hline 1 & No & Yes & 1 & 4 & 20 & 0,361 & 0,887 & 0,985 & 2,456 & 2,726 \\
\hline 2 & No & Yes & 1 & 4 & 15 & 0,344 & 0,885 & 0,982 & 2,570 & 2,852 \\
\hline 3 & No & Yes & 1 & 4 & 10 & 0,316 & 0,877 & 0,973 & 2,777 & 3,082 \\
\hline 4 & No & Yes & 1 & 4 & 7 & 0,286 & 0,867 & 0,962 & 3,027 & 3,359 \\
\hline 5 & No & Yes & 1 & 4 & 5 & 0,256 & 0,854 & 0,947 & 3,340 & 3,706 \\
\hline 6 & No & Yes & 1 & 6 & 20 & 0,338 & 0,883 & 0,980 & 2,610 & 2,897 \\
\hline 7 & No & Yes & 1 & 6 & 15 & 0,315 & 0,878 & 0,974 & 2,788 & 3,093 \\
\hline 8 & No & Yes & 1 & 6 & 10 & 0,276 & 0,865 & 0,960 & 3,136 & 3,481 \\
\hline 9 & No & Yes & 1 & 6 & 7 & 0,237 & 0,849 & 0,943 & 3,580 & 3,973 \\
\hline 10 & No & Yes & 1 & 6 & 5 & 0,199 & 0,828 & 0,918 & 4,166 & 4,623 \\
\hline 11 & No & Yes & 1 & 8 & 20 & 0,308 & 0,877 & 0,973 & 2,844 & 3,156 \\
\hline 12 & No & Yes & 1 & 8 & 15 & 0,278 & 0,868 & 0,963 & 3,117 & 3,459 \\
\hline 13 & No & Yes & 1 & 8 & 10 & 0,231 & 0,851 & 0,944 & 3,687 & 4,092 \\
\hline 14 & No & Yes & 1 & 8 & 7 & 0,187 & 0,829 & 0,920 & 4,434 & 4,920 \\
\hline 15 & No & Yes & 1 & 8 & 5 & 0,146 & 0,797 & 0,884 & 5,453 & 6,051 \\
\hline 16 & No & Yes & 1 & 10 & 20 & 0,276 & 0,865 & 0,960 & 3,130 & 3,473 \\
\hline 17 & No & Yes & 1 & 10 & 15 & 0,240 & 0,853 & 0,946 & 3,552 & 3,941 \\
\hline 18 & No & Yes & 1 & 10 & 10 & 0,187 & 0,830 & 0,921 & 4,438 & 4,925 \\
\hline 19 & No & Yes & 1 & 10 & 7 & 0,142 & 0,801 & 0,889 & 5,642 & 6,261 \\
\hline 20 & No & Yes & 1 & 10 & 5 & 0,105 & 0,752 & 0,834 & 7,170 & 7,956 \\
\hline 21 & No & Yes & 1 & 10 & 3 & 0,060 & 0,603 & 0,669 & 9,990 & 11,086 \\
\hline 22 & Yes & Yes & 1 & 4 & 20 & 0,232 & 0,791 & 0,877 & 3,406 & 3,780 \\
\hline 23 & Yes & Yes & 1 & 4 & 15 & 0,225 & 0,789 & 0,875 & 3,500 & 3,884 \\
\hline 24 & Yes & Yes & 1 & 4 & 10 & 0,213 & 0,784 & 0,870 & 3,680 & 4,084 \\
\hline 25 & Yes & Yes & 1 & 4 & 7 & 0,199 & 0,778 & 0,863 & 3,906 & 4,335 \\
\hline 26 & Yes & Yes & 1 & 4 & 5 & 0,184 & 0,769 & 0,854 & 4,193 & 4,653 \\
\hline 27 & Yes & Yes & 1 & 6 & 20 & 0,223 & 0,788 & 0,875 & 3,531 & 3,918 \\
\hline 28 & Yes & Yes & 1 & 6 & 15 & 0,213 & 0,785 & 0,871 & 3,684 & 4,088 \\
\hline 29 & Yes & Yes & 1 & 6 & 10 & 0,194 & 0,777 & 0,862 & 4,001 & 4,440 \\
\hline 30 & Yes & Yes & 1 & 6 & 7 & 0,174 & 0,768 & 0,852 & 4,421 & 4,906 \\
\hline 31 & Yes & Yes & 1 & 6 & 5 & 0,151 & 0,751 & 0,833 & 4,976 & 5,522 \\
\hline 32 & Yes & Yes & 1 & 8 & 20 & 0,210 & 0,785 & 0,871 & 3,732 & 4,142 \\
\hline 33 & Yes & Yes & 1 & 8 & 15 & 0,196 & 0,780 & 0,865 & 3,982 & 4,419 \\
\hline 34 & Yes & Yes & 1 & 8 & 10 & 0,170 & 0,769 & 0,853 & 4,519 & 5,015 \\
\hline 35 & Yes & Yes & 1 & 8 & 7 & 0,144 & 0,753 & 0,836 & 5,244 & 5,820 \\
\hline 36 & Yes & Yes & 1 & 8 & 5 & 0,116 & 0,728 & 0,807 & 6,275 & 6,964 \\
\hline 37 & Yes & Yes & 1 & 10 & 20 & 0,195 & 0,777 & 0,863 & 3,980 & 4,417 \\
\hline 38 & Yes & Yes & 1 & 10 & 15 & 0,176 & 0,770 & 0,855 & 4,375 & 4,856 \\
\hline 39 & Yes & Yes & 1 & 10 & 10 & 0,144 & 0,753 & 0,836 & 5,244 & 5,819 \\
\hline 40 & Yes & Yes & 1 & 10 & 7 & 0,113 & 0,732 & 0,812 & 6,472 & 7,183 \\
\hline 41 & Yes & Yes & 1 & 10 & 5 & 0,085 & 0,688 & 0,764 & 8,070 & 8,955 \\
\hline 42 & Yes & Yes & 1 & 10 & 3 & 0,050 & 0,555 & 0,615 & 11,104 & 12,323 \\
\hline 43 & No & Yes & 2 & 4 & 20 & 0,224 & 0,684 & 0,734 & 3,058 & 3,278 \\
\hline 44 & No & Yes & 2 & 4 & 10 & 0,179 & 0,654 & 0,701 & 3,660 & 3,924 \\
\hline 45 & No & Yes & 2 & 8 & 7 & 0,081 & 0,564 & 0,605 & 6,950 & 7,450 \\
\hline 46 & No & Yes & 2 & 8 & 10 & 0,115 & 0,606 & 0,650 & 5,288 & 5,669 \\
\hline 47 & No & Yes & 2 & 10 & 5 & 0,030 & 0,419 & 0,450 & 14,020 & 15,030 \\
\hline 48 & No & Yes & 2 & 10 & 3 & 0,011 & 0,241 & 0,259 & 22,563 & 24,189 \\
\hline
\end{tabular}

Table 4.5.: Ar+KCl@1,756 AGeV: Efficiency and Enhancement by Correlation Parameter.

This Table shows a systematic calculation of efficiency and enhancement for different settings of the correlation parameters. The L3 column denotes if the RICH to MDC correlation trigger was active, while L2 denotes the same for the HADES level 2 trigger system. Multiplicity (M) is the minimum number of required good leptons per event. Result 0 shows the results of the HADES level 2 trigger for the same data set for comparison. From this table and the discussion of the previous distributions the trigger setting would be 10 layers and 7 cells (Result: 19). This parameter set should be cross referenced with the Efficiency distributions in Figure 4.31, Figure 4.32 and Figure 4.33. The values of eff2 and enh2 demonstrate the influence of the RICH IPU (compare Section 4.4.4). 

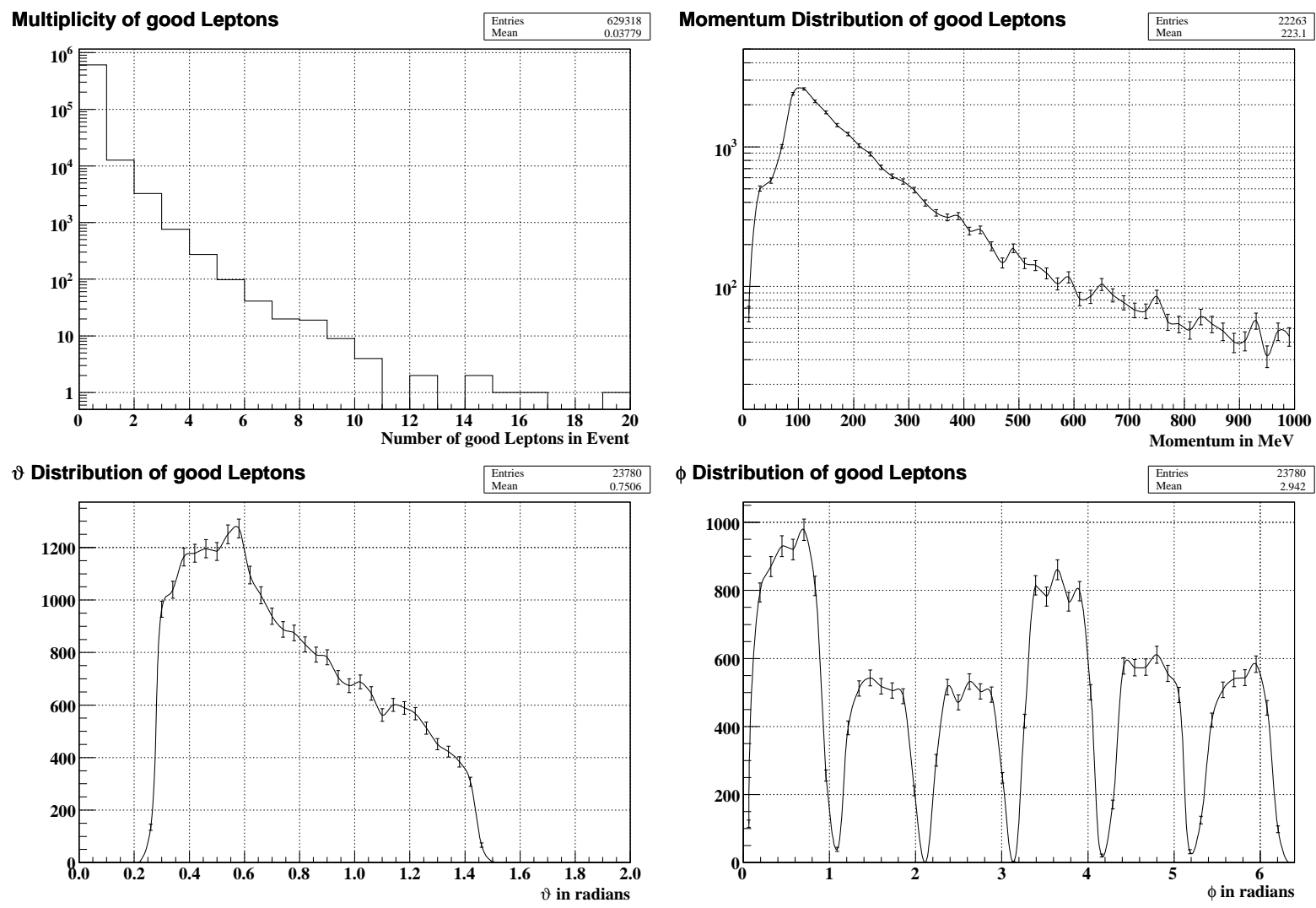

Figure 4.30.: Ar+KCI@1,756 AGeV: Good Lepton Base Data.

The histograms show the base data of the good leptons. Their multiplicity per event histogram shows how few events actually contain one or more good lepton. The $\vartheta$-distribution and the $\phi$ distribution show the respective angels of the lepton before the magnetic field and clearly follow the geometrical structure of the HADES detector. The momentum distribution is obtained from the "spline" algorithm, using the bending of the track in the magnetic field. These distributions provide the good total events in Equation (4.10). Dividing the accepted good lepton distributions for different correlation parameters with these histograms gives the different efficiency distribution histograms. It should be noted that the statistics are not very large for momenta above $500 \mathrm{MeV}$. The errors are deduced from the statistical error only. 

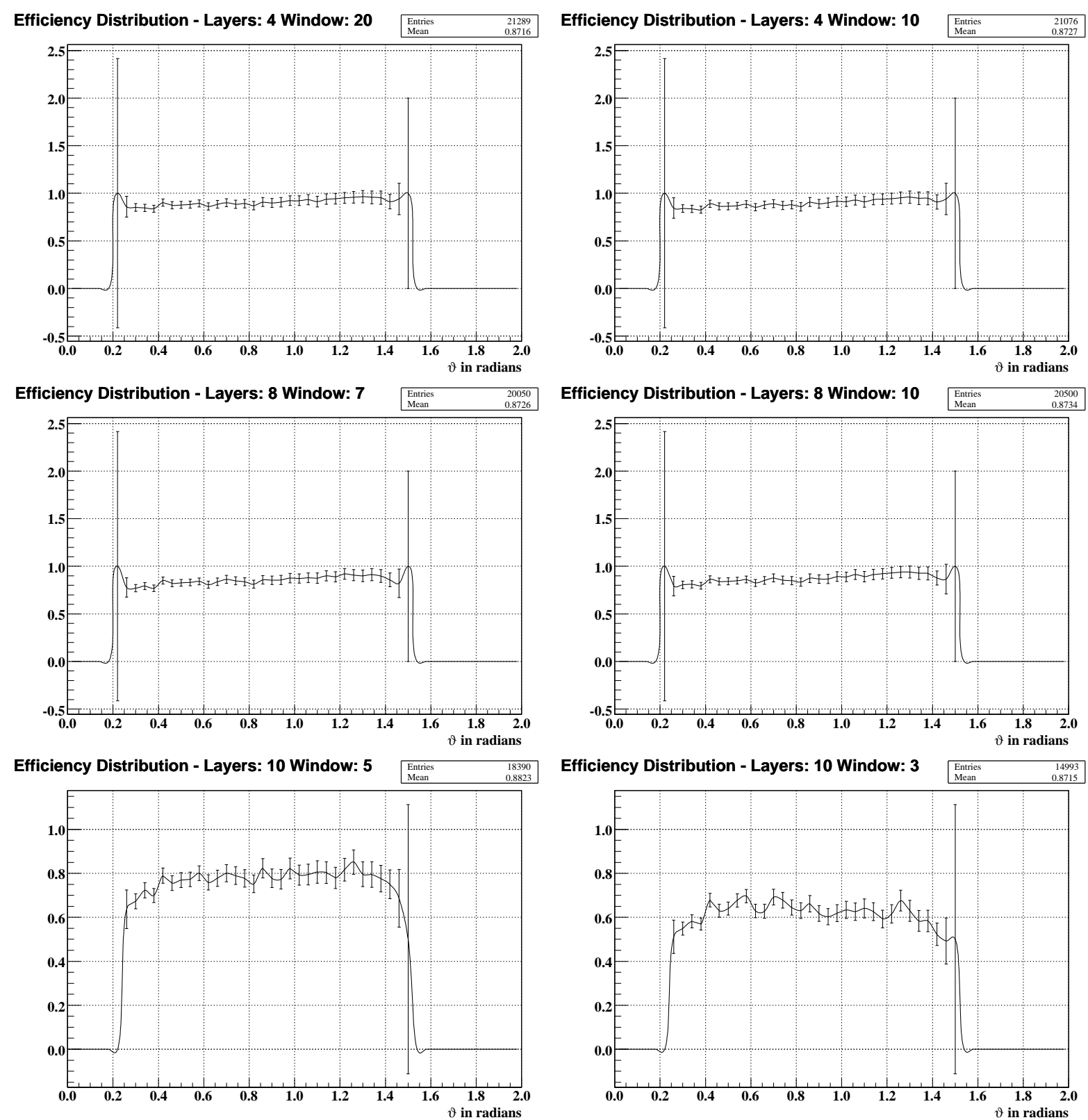

Figure 4.31.: Ar+KCI@1,756 AGeV: $\vartheta$ - Efficiency Distributions

These histograms show the efficiency as a function of the $\vartheta$-angle eff $(\vartheta)$ as defined in Section 4.4.2 for several selected settings of the correlation algorithm. The distributions are very flat up to a setting of 8 layers and a window of 10 cells, only a very smooth rise is visible. For more aggressive trigger settings the distributions start to show distortions. The large error bars are at the position of the edges of the detector. 
Efficiency Distribution - Layers: 4 Window: 20

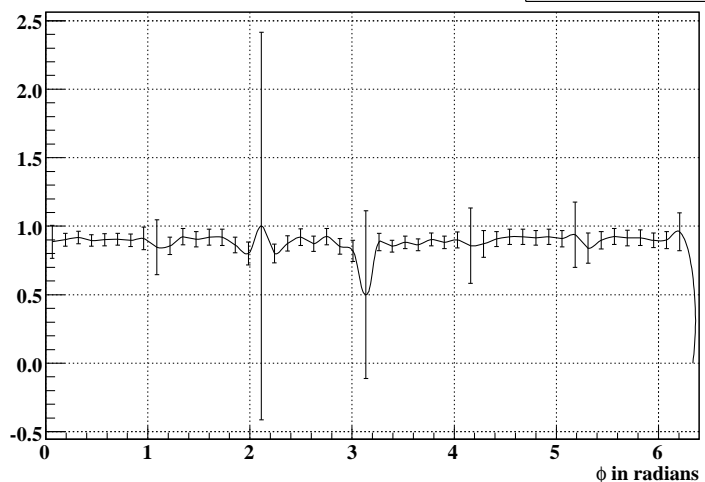

Efficiency Distribution - Layers: 8 Window: 7

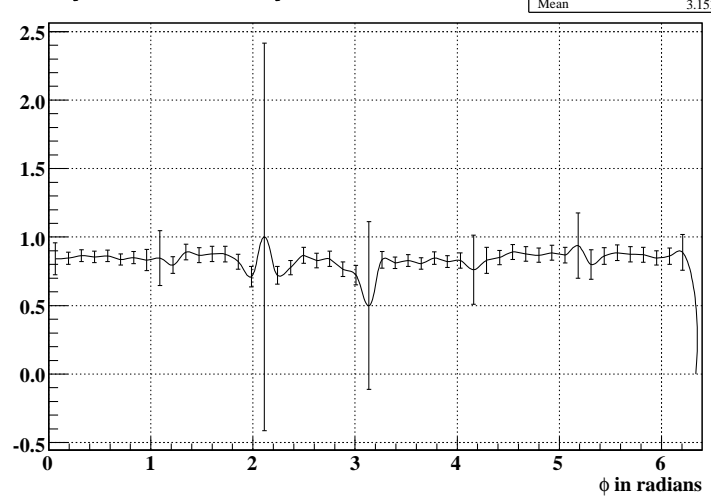

Efficiency Distribution - Layers: 10 Window: 5

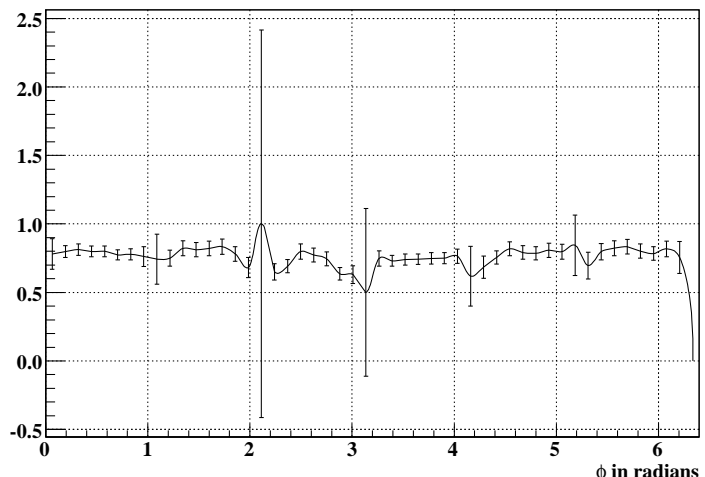

Efficiency Distribution - Layers: 4 Window: 10

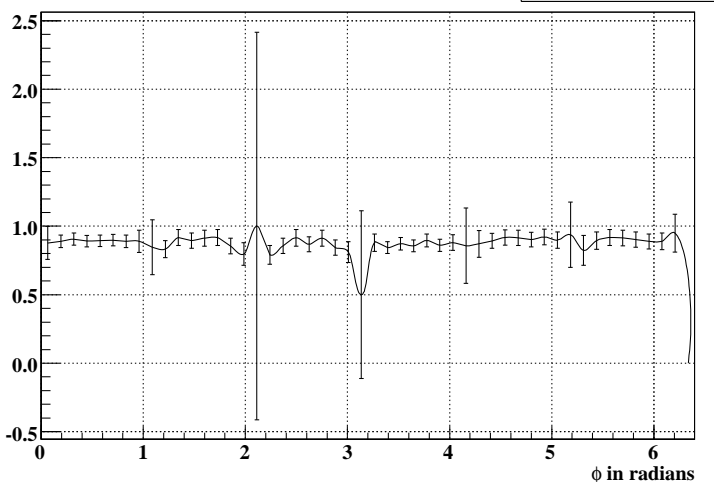

Efficiency Distribution - Layers: 8 Window: 10

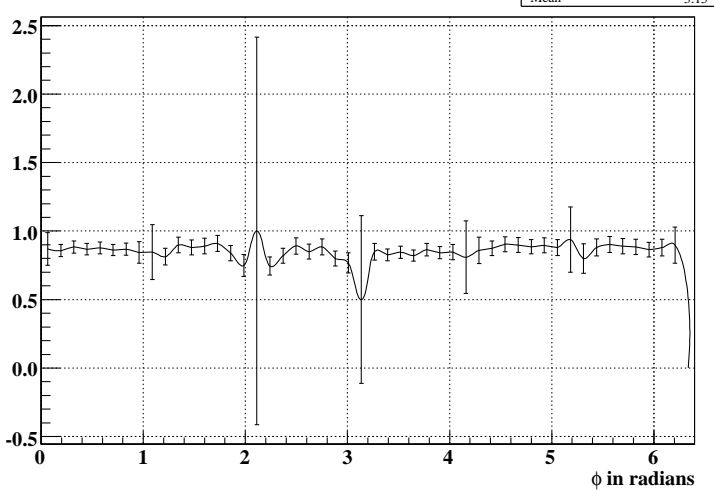

Efficiency Distribution - Layers: 10 Window: 3

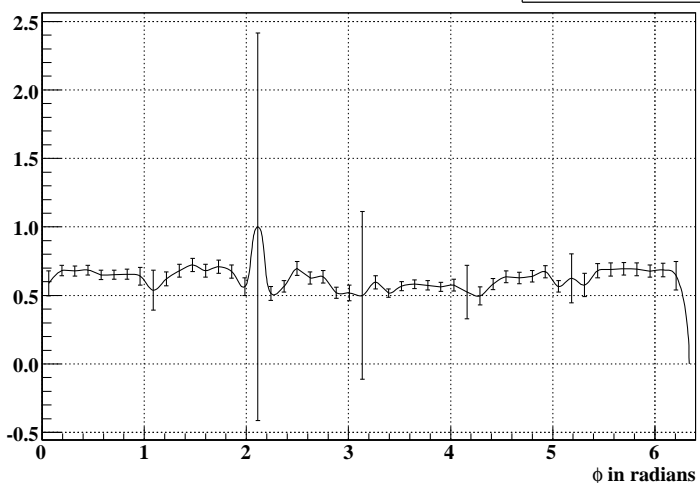

Figure 4.32.: Ar+KCl@1,756 AGeV: $\phi$ - Efficiency Distributions

These histograms show the efficiency as a function of the $\phi$-angle eff $(\phi)$ as defined in Section 4.4.2 for several selected settings of the correlation algorithm. The distributions are very flat up to a setting of 8 layers and a window of 10 cells. For more aggressive trigger settings the distributions start to show distortions. The large error bars are at the position of the edges of the detector. 

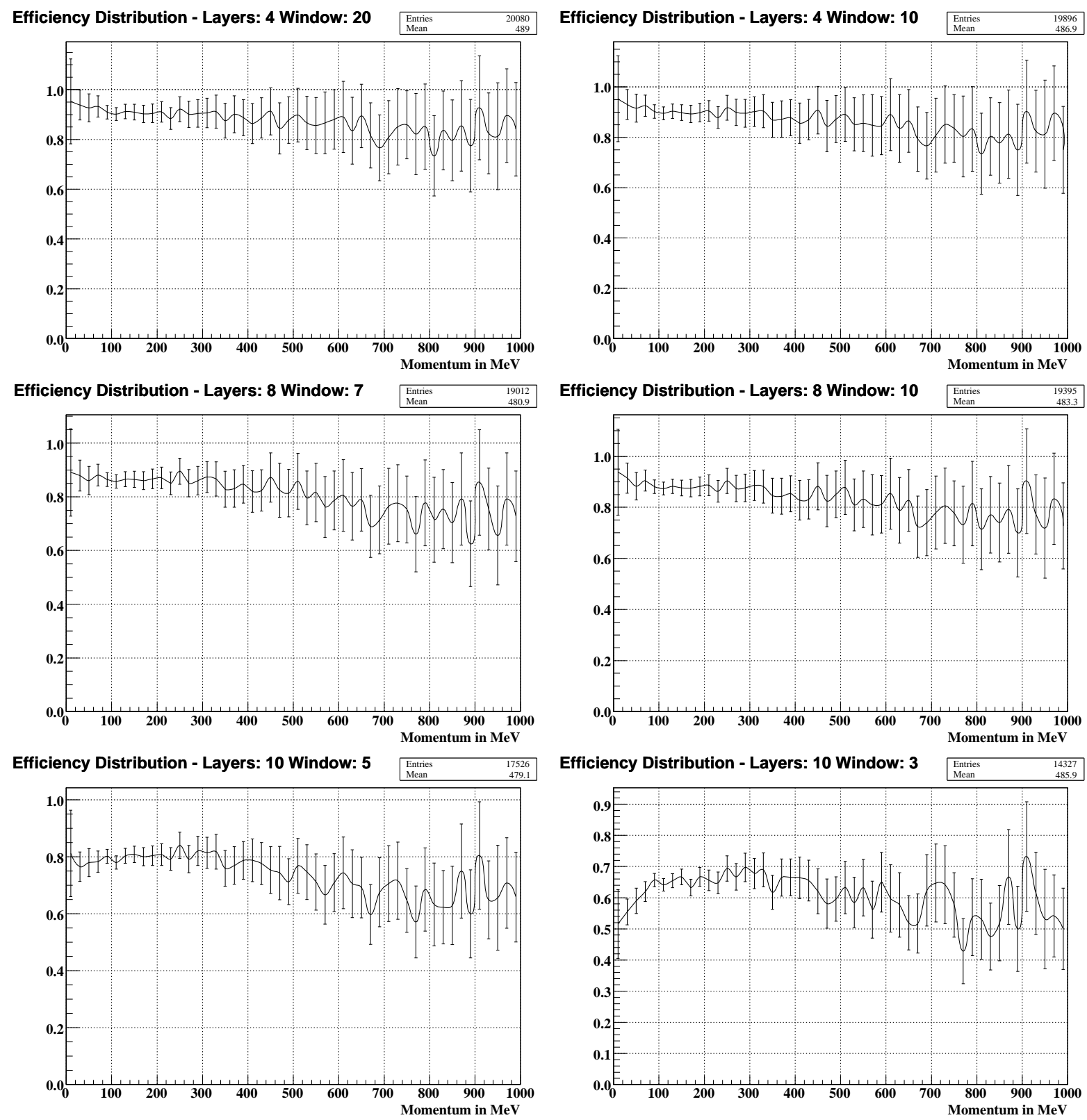

Figure 4.33.: Ar+KCl@1,756 AGeV: Momentum Efficiency Distributions

These histograms show the efficiency as a function of the momentum eff $(p)$ as defined in Section 4.4.2 for several selected settings of the correlation algorithm. The statistics for good leptons is reasonable in this data set for momenta up to $500 \mathrm{MeV}$ and up to this value the distributions are very flat up to trigger settings of setting of 8 layers and a window of 10 cells. Only a smooth drop is hinted. For more aggressive trigger settings the distributions start to show distortions. 


\subsection{Bibliography}

[Bru97] Rene Brun and Fons Rademakers; ROOT - An object oriented data analysis framework (1997); (389):81-86; doi:10.1016/S0168-9002(97)00048-X

[Bru07] Rene Brun and Fons Rademakers; The ROOT Website (2007); URL http:// root.cern.ch

[HADa] HADES Simulation and Analysis Website; URL http://www-hades.gsi.de/ computing/

[Leh00] JÖRG LEHNERT (HADES); Echtzeit-Mustererkennung zum Elektronennachweis mit einem RICH-Detektor in relativistischen Schwerionenkollisionen; Ph.D. thesis; II. Physikalisches Institut der Justus-Liebig-Universität Gießen (June 2000); URL http: //pcweb.physik.uni-giessen.de/exp2

[Ora06] Oracle Corporation; The Oracle Website (2006); URL http://www.oracle.com

[SG03a] Manuel Sánches García; Momentum Reconstruction and Pion Production in the HADES Spectrometer at GSI; Ph.D. thesis; Universidade de Santiago de Compostela, Facultad de Física (April 2003)

[Toi04] Alberica TOIA (HADES); Performance of the HADES Spectrometer for Dilepton Identification in the Reaction $C+C$ at $1 \mathrm{AGeV}$; Ph.D. thesis; II. Physikalisches Institut der Justus-Liebig-Universität Gießen (March 2004); URL http://pcweb . physik. uni-giessen.de/exp2 


\section{Summary, Discussion and Outlook}

\section{Contents}

5.1. RICH to MDC Correlation Summary . . . . . . . . . . . . 129

5.2. Discussion of the RICH to MDC Correlation Method . . . . . . 130

5.2.1. Beamtime Comparison: $\mathrm{p}+\mathrm{p}$ vs $\mathrm{C}+\mathrm{C}$ vs $\mathrm{Ar}+\mathrm{KCl} \ldots \ldots \ldots \ldots 131$

5.2.2. Outlook: RICH to MDC Correlation with other Algorithms . . . . . . 132

5.2.3. Outlook: Correlating the outer MDC Chambers . . . . . . . . . 132

5.3. Discussion of the RICH to MDC Correlation Performance . . . . 133

5.3.1. Performance in the GE-MN . . . . . . . . . . . . . . . . 134

5.3.2. Performance Scenarios with HADES . . . . . . . . . . . . . . . 134

5.4. GE-MN Technical Summary and Outlook . . . . . . . . . 135

5.4.1. Directly Related Projects . . . . . . . . . . . . . . . . . 136

5.4.2. The New HADES DAQ and Trigger System . . . . . . . . . . 136

5.4.3. RICH to MDC Correlation in the new HADES Trigger . . . . . . . . 139

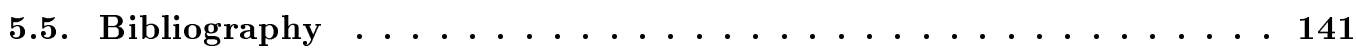

This chapter discusses the results of the previous chapters. In addition, it provides a compact summary of the results obtained and discussed in the previous chapters. The possible next steps in the application of the RICH to MDC correlation method are sketched in the respective outlook sections (Section 5.2.2 and Section 5.2.3). Further, the relations and the connections of this work to other ongoing projects and developments are also summarized.

\subsection{RICH to MDC Correlation Summary}

The trigger method presented in Chapter 4 correlates the ring information as delivered by the RICH IPU with the raw wire (cell) data from the MDC detector. It operates by investigating the correlation between the MDC cells which should have seen a hit with MDC cells which actually did detect a signal. The MDC cells which should have fired are calculated by taking the ring $(\vartheta ; \phi)$-angular information from the RICH IPU and forming a straight line in space, which is starting from the target with these angles. The line extends through the inner MDC chambers and the traversed cells are those that should have been hit. To compensate different sources for inaccuracies not the exact cell numbers are used. Instead the cells in a matching window are investigated. In addition, only a fraction of the MDC layers that should be hit are required to have seen a hit.

It has been demonstrated that this RICH IPU ring to MDC cell correlation trigger method does yield good results for light $(\mathrm{p}+\mathrm{p}$ ) and medium ( up to $\mathrm{C}+\mathrm{C}$ ) sized collision system (details in Section 5.2). The achievable enhancements can be larger than 50, if small targets are used. It has been shown that even at these high enhancements the efficiency remains at a high level 
(above $80 \%$ ). The main source of the drop in efficiency has been identified to be the RICH IPU efficiency, which is consistent with other investigations (compare [Toi04]).

The investigated heavy system $\mathrm{Ar}+\mathrm{KCl}$ shows that the correlation method is also working there. The achievable enhancement is $\approx 5$, but the efficiency is still above $80 \%$. Determining the usable trigger settings is more involved as higher statistics are needed - a factor of 10 more is required compared to $\mathrm{C}+\mathrm{C}$ data.

In all presented data analysis the same systematic procedure has been applied to deduce the usable trigger parameters from online available data only (i.e. the raw data available at data recording) and in all cases the full offline data analysis verified successfully the quality of the deduced settings.

The dependence of the efficiency on physics parameters, in particular the momentum, has been studied and all the dependencies have been found to be flat for the selected trigger parameters.

The performance of the correlation method in terms of the analyzed events per second has been studied with the GE-MN (compare Chapter 3). The performance depends on the input data and ranges form 6600 events/s for $\mathrm{p}+\mathrm{p}$ data to 1400 events/s for $\mathrm{Ar}+\mathrm{KCl}$ data for a single GE-MN system.

\subsection{Discussion of the RICH to MDC Correlation Method}

The correlation method presented in Chapter 4 has been applied to several data sets from different beamtimes to cover all reaction types measured so far by the HADES experiment. The following reactions where measured so far: $\mathrm{p}+\mathrm{p}, \mathrm{C}+\mathrm{C}$ and $\mathrm{Ar}+\mathrm{KCl}$. The detailed investigations can be found in Section 4.4.5, Section 4.4.6 and Section 4.4.7. They show that the RICH to MDC correlation method does work well for collision systems up to $\mathrm{C}+\mathrm{C}$ and can reach enhancements of up to a factor of 50 at efficiencies above $80 \%$.

The investigation of the online histograms (Figure 4.6, Figure 4.15) immediately shows a strong correlation peak, which can be used to set and check the width of the correlation window online (Section 4.2). The offline comparison of the same histograms for good lepton events (Figure 4.7, Figure 4.18, Figure 4.27) shows, that indeed the good leptons are triggered. A similar procedure applied to the histograms of the number of matched layers (Figure 4.8, Figure 4.19, Figure 4.28) allows to deduce the setting of the required minimum number of matched layers. Likewise, the same histograms for the good lepton events (Figure 4.9, Figure 4.20, Figure 4.29) prove, that indeed the good lepton events are selected. The definition of a "good lepton" and a good lepton event can be found in Figure 4.1.

It is important to stress that the trigger parameters for the correlation method can be deduced directly from the raw data while the experiment is running. It has been demonstrated, that no high level offline analysis is required to deduce the settings. In addition, a universal safe setting is available for all trigger parameters. There, the required number of layers is set to 4 and the matching window to 20 cells. The layer cut is safe, because of the properties of the offline cluster finder (discussed in Section 2.11 and Section 4.2.1). The matching window of 20 cells is simply wide enough to accept anything which could possibly have a correlation.

The systematic investigations of the influence of different parameter sets show, that the deduced parameter sets are not significantly cutting into the efficiency. The reduction also remains at a high level. Thus, the enhancement is high and is not many good lepton events are lost. The comparison with the second level trigger performance shows, that the correlation of RICH IPU rings with MDC cells is the more selective trigger method, and that it has a better efficiency. In 
addition, it is shown that both methods can be combined. In this case the efficiencies roughly add up, since both methods correlate the data of different detectors. In this discussion it should be mentioned, that the efficiency of the presented method is clearly dominated by the efficiency of the RICH IPU (e.g eff2 in Table 4.4) and not by the correlation itself. Thus, the availability of RICH IPU data with a higher efficiency would also be a big improvement. Unfortunately, a complete redesign of the RICH IPU system would be necessary to provide this. A very important question for a trigger method is, if it is introducing unwanted structures (a bias) in the acceptance of a detector system. It has been demonstrated that this is not the case by studying the angular efficiency distributions and momentum efficiency distributions. The efficiency distributions are flat for the deduced settings, and only much stronger cuts distort the efficiency.

\subsubsection{Beamtime Comparison: $\mathrm{p}+\mathrm{p}$ vs $\mathrm{C}+\mathrm{C}$ vs $\mathrm{Ar}+\mathrm{KCl}$}

Comparing the results for $\mathrm{p}+\mathrm{p}$ data (Section 4.4.5) with the results for $\mathrm{C}+\mathrm{C}$ data (Section 4.4.6) a remarkable feature is visible. The $\mathrm{C}+\mathrm{C}$ data set has a significantly more narrow correlation peak (compare Figure 4.17), while it is the heavier system with much higher multiplicity and background. One reason is that the carbon target, with a thickness of only $6,5 \mathrm{~mm}$, is much smaller as the liquid hydrogen target, which is $5 \mathrm{~cm}$ thick. Because of this, the start point for the RICH IPU ring to MDC cell correlation is much less point-like in the LH2 target case. This results in a wider correlation peak. An other reason is, that the proton beam is hitting material belonging to beam-pipe system, and by this it is producing an unwanted halo. In the April 2007 beamtime this effect has been reduced by changing the target setup. A check of this data (not presented here) did show a slightly more narrow correlation peak and somewhat reduced background, but the changes seen in the correlations plots are not very large. The conclusion is, that this correlation method works better for thin targets, but it is also working with an increased matching window size for large targets. The actual improvement is shown in the table of the different parameter settings in Table 4.3 and in Table 4.4. It can be up to a factor of two in enhancement. The increased combinatorial background of the $\mathrm{C}+\mathrm{C}$ data is clearly visible in the comparison of Figure 4.17 with Figure 4.6.

For the $\mathrm{Ar}+\mathrm{KCl}$ data discussed in Section 4.4.7 the situation is different. There, already the initial correlation histograms make the deduction of a narrow matching window difficult. The statistics from several runs is required to see the correlation peak in sufficient detail to deduce the correlation parameters. About a factor of $\approx 10$ more statistics is needed in relation to the $\mathrm{C}+\mathrm{C}$ data. It seems that the combinatorial background shadows much of the correlation peak. The histograms for the good lepton data are also not very satisfying. They still clearly show the presence of a sharp correlation in the data, but they also show a strong background (compare Figure 4.27), which might indicate a problem in the good lepton definition for this data. The statistics, especially for the good lepton events, is also very low when only one run is investigated. This is obviously also the case for all derived quantities like efficiency and enhancement. It seems that one reason of these problems is the much larger number of possible combinations between RICH IPU rings and fired MDC cells. This is discussed in Figure 5.1.

The enhancement of the RICH to MDC correlation method is not as high as when applied to the lighter systems. On the other hand it is still significantly better than the HADES second level trigger, because the efficiencies are comparable and the reduction is about a factor of two larger. No serious problems can be identified in the efficiency distributions, but with the limited statistics it is difficult to draw definitive conclusions for good lepton momenta above $500 \mathrm{MeV}$. 

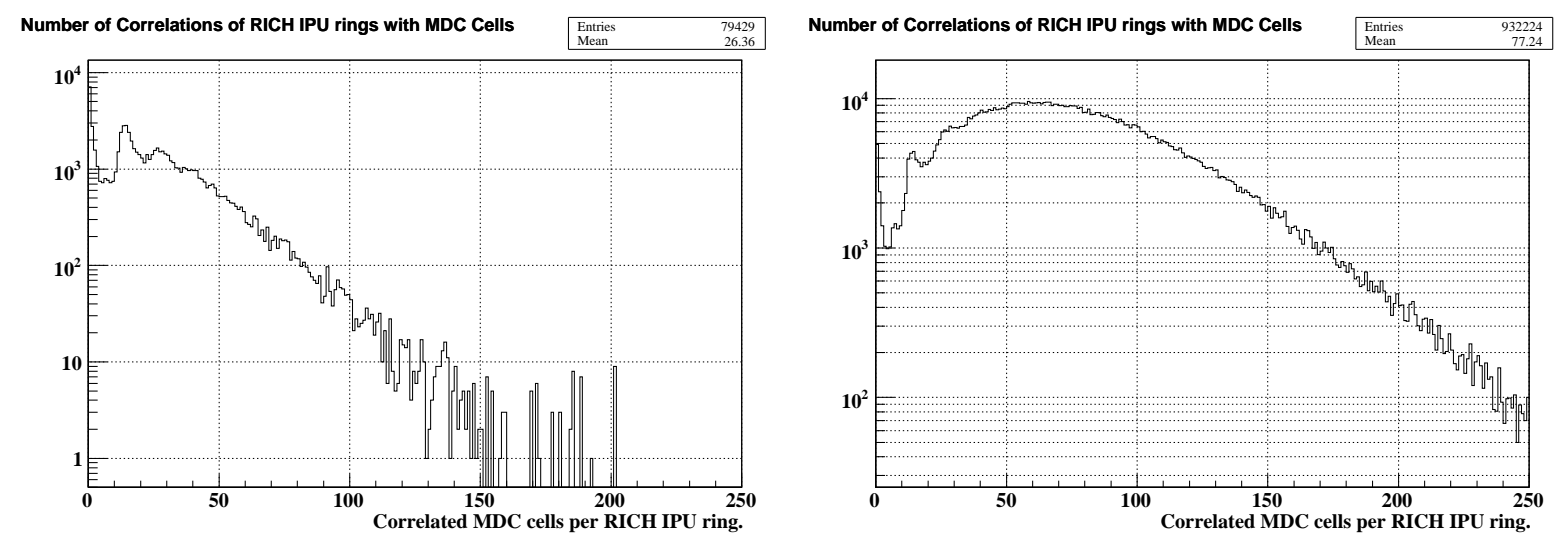

Figure 5.1.: Number of Correlations Performed between RICH IPU Rings and MDC Cells.

The histogram on the left is shows $\mathrm{C}+\mathrm{C}$ data, while the histogram on the right side shows $\mathrm{Ar}+\mathrm{KCl}$ data. The $\mathrm{Ar}+\mathrm{KCl}$ data samples have a much higher average number of correlations $(\approx 90)$ than the $\mathrm{C}+\mathrm{C}$ data $(\approx 26)$. Clearly visible in the data sets are peaks around 12 . This peak is expected from good tracks hitting all the 12 layers. A peak around 24 is expected from events with two good tracks, but it is barley visible here.

\subsubsection{Outlook: RICH to MDC Correlation with other Algorithms}

The presented analysis of the correlation method are discussed in terms of accepted and rejected events, or in other words as a standalone trigger method. There is no reason not to combine the RICH to MDC correlation with other methods, also on the base of individual RICH rings. For instance, it would be a sensible approach to combine the correlation method with the HADES second level trigger, and to execute latter only for RICH IPU rings which passed the former algorithm. Thus, the load of the HADES second level trigger would be reduced.

It is also conceivable to include the correlation method into the software framework. A large fraction of processing time in the offline analysis is used for the different track reconstruction methods, i.e. cluster finder, kick-track approach, spline tracking and RUNGE-KUTTA tracking (compare Section 2.11). If only lepton tracks are of interest, the processing time for these algorithms can be significantly reduced by executing the full tracking procedure only for events which have a high probability of having a lepton, i.e. events triggered positively by the RICH to MDC correlation.

\subsubsection{Outlook: Correlating the outer MDC Chambers}

The enhancement of lepton tracks in the HADES experiment could be increased further by extending the investigated correlation method to the detectors behind the magnetic field. The correlation method needs a straight line in space which can be checked for consistency with a track by investigating if enough MDC layers have been hit in the matching window. This straight line can be defined by two points (compare Figure 2.2). On one side a hit in the META system defines a precise point in space. The point on the other side can be defined by the cross point of a assumed track with the kick plane. The straight line representing this assumed track can be defined by the target and a hit in the RICH, in the very same way as it is done for the inner MDC correlation, i.e. the track starts at the nominal target position and its direction is defined 
by the $(\vartheta, \phi)$ information of a RICH ring. The cross-point with the kick plane is not extremely well defined, because the $(\vartheta, \phi)$ information from a RICH ring is not very precise (discussed in Section 4.2.1). Since it is not a priori clear which META hit should be combined with which kick plane cross-point, all combinations have to be checked. Accepting only combinations of positively matched inner (RICH with inner MDC) and outer (kick plane with outer MDC) combinations provides a way of enriching the content of good leptons in the data even further.

The extended method is approximately twice as expensive in required compute power as the original method, since the number of combinations of kick plane hits with meta hits should be about as high as number of the fake RICH hits which have to be correlated with the inner MDC chambers in the original method. As such, it is feasible from the needed amount of compute power. In addition to the enhanced enrichment the correlated inner and outer matches also provides an estimation of particle momentum as well as the polarity of the particle. Thus, even a particle identification for electrons and positrons is provided. The HADES second level trigger would be automatically implicitly included in this extended method.

The crucial question for the feasibility of this extended method is, if the crossed point of the kick plane is well enough defined to allow reasonable small matching window for the outer MDC correlation. Another important question is, if the number of combinations between META hits and kick plane hits is really not exceedingly high, and how the requirements of the method grow when heavy systems are investigated. The most important step in implementing this matching of outer layers is the development of a function calculating the crossed outer MDC cells along a straight line. The kick plane related topics have been already addressed in the matching unit implementation (described in [Tra01]) and could be used.

\subsection{Discussion of the RICH to MDC Correlation Performance}

The performance of the described correlation method in terms of processed events/second has been investigated with the GE-MN (compare Chapter 3). This provides real live results for the performance and enables to judge the processing power needed for the algorithm when used in an online trigger system. The correlation (trigger) algorithm discussed in the previous sections performs differently when used to process data from different physical processes. The reason for this is primarily the different multiplicities of different experiments. In $p+p$ reactions the average multiplicity is very low and thus only few charged particles can produce hits in the MDC chambers. Also the number of rings in the RICH is lower. These change the performance in two algorithmic ways: For each RICH IPU ring the crossed cell for each of the 12 inner MDC layers has to be calculated. This is a very fast procedure as can be seen in the description in Section 4.3.1 and involves per layer only a few look-ups and few calculations.

A significantly larger amount of time is spent in the actual comparison of the calculated cell numbers with all the real hit cells, which also have to be translated from their motherboard and TDC number $(m b o ; t d c)$ address into the layer and cell number address (lay; cel) of actual interest. Thus, the dominant algorithmic influence is the pure number of MDC hit cells which have to be correlated. In addition, the performance of the GE-MN also depends on the amount of data which has to be transmitted to execute the correlation. Also in this respect the MDC cell data samples are largely dominant and have an impact on the performance. It should also be noted that the trigger parameters (required number of layers and matching window) have only very little impact on the performance, since the way they enter the performance is that the correlation does not need to be continued when a sufficient number of layers have been matched 
in an event. It should be stressed that the reason this method is fast is that it does not do any track reconstruction from the wire chamber data, but just checks the compatibility of the correlation result with the necessary minimum requirements for the presence of a track - a minimum number of fired cells in a certain MDC region given by the RICH IPU ring.

\subsubsection{Performance in the GE-MN}

The performance of the processing has been investigated by packing the RICH IPU $(\theta, \phi)$ information and the MDC fired wire data from one event into an UDP Datagram and sending it to the GE-MN for processing. It is interesting to note that if enough data samples have to be transmitted, the Datagram has to be split up among several network packets (fragmentation; compare Section 3.2.3). The results of the correlation are then send back to a PC. The maximum performance is as expected measured for $\mathrm{p}+\mathrm{p}$ data. Here the average packet size is only 240 Octets and the average fragmentation ratio is $0,1 \%$. The event rate per second is 6600 events $/ \mathrm{s}$ and thus the data rate is $1,6 \mathrm{MB} / \mathrm{s}$. The lowest performance is measured, as expected, for $\mathrm{Ar}+\mathrm{KCl}$ data. Here, the fragmentation rate is $66 \%$ at an average packet size of 1800 Octets, since the amount of MDC wire data is so high. This leads to a average processing speed of 1400 events/s at a data rate of $2,5 \mathrm{MB} / \mathrm{s}$. All these performance figures are for a single GE-MN system.

\subsubsection{Performance Scenarios with HADES}

The investigated correlation method has basically two options of possible usage in HADES. It can process the event data either before or after the HADES second level trigger. Both options have been investigated in the individual data analysis sections and the results are discussed in Section 4.4. For the average processing time required it is relevant how many events the HADES second level trigger has to process during a spill. This is given by the average rate the first level trigger delivers data to the second level trigger. The maximum average rate has been about $\approx 10 \mathrm{kHz}$ for elementary reactions and $\approx 5 \mathrm{kHz}$ for heavy ion reactions in the beamtimes so far [Tra07]. The momentary rates during a spill, which are also accepted by the second level trigger, are twice these average numbers. These high instantaneous rates are caused by the sub-structure of the beam (micro spill structure). It is worth to note that the rate limitations limit was not only imposed by the second level trigger, but also by a combination of the detectors and their readout. Comparing these maximum average rate to the performance of the correlation method in the GE-MN it is clear that a system like the GE-MN is always fast enough to handle the data after the second level trigger, if sufficient buffering is available to decouple from instantaneous rate effects. Processing data before or instead of the second level trigger needs more performance. In the $\mathrm{Ar}+\mathrm{KCl}$ beamtime the maximum average event rate delivered to the second level trigger was $4 \mathrm{kHz}$.

Two basic options are available to increase the performance. First, the performance can be increased by using faster signal processors (DSP), which are easily available now and give a performance increase of about a factor of two to three. The other option is to use more than one system to process the data. In this case a natural partitioning of the multiprocessor system is given directly by the algorithm and the detector geometry: The method correlates RICH IPU rings with MDC cells of the same sector. Thus a natural partitioning would be one correlation system per sector, which improves the performance directly by a factor of six. This is easily sufficient also for heavier system and can be combined with the first option. For this combined 
option a processing speed of about $2 \times 6 \times 1400$ events $/ \mathrm{s}=16,8 \mathrm{k}$ events $/ \mathrm{s}$ could be reached. This can be further improved by sub-partitioning into chambers, which is discussed in Section 5.4.3. It should be noted, that it would be most natural to perform the correlation at a place where all the required MDC data of a sector and sufficient compute power is available by default, instead of (as demonstrated here with the GE-MN) shipping all the data to another central place for processing.

\subsection{GE-MN Technical Summary and Outlook}

The GE-MN (Chapter 3) is a data processing system developed for high level trigger applications at accelerator experiments. It features two Gigabit-Ethernet network interfaces, a VMEbus and a signal processor. The main interfaces to receive and send analysis data are the Gigabit-Ethernet interfaces, while the VMEbus provides slow control, boot-up, configuration and monitoring. Monitoring can be done out of band and in parallel to ongoing data transfer on the network interfaces. The processing of data is performed by the TigerSHARC digital signal processor.

To demonstrate the capabilities of the GE-MN a trigger algorithm in the context of the HADES experiment was chosen as an example: The real time correlation of RICH IPU ring data with the fired wire data of the MDC wire chambers as summarized in Section 5.1.

The GE-MN is the prototype of an Ethernet based general purpose data processing solution, and its development provided insight into the different challenges of implementing such a system. The actual hardware had to be designed and built, and to get the basic functionality of data transfer and bus accesses this hardware also had to be programmed. The main hardware programming challenge is the implementation of the data transport on the various interfaces in VHDL. The software layer on top of the hardware, the program executed in the DSP, has to perform the actual application tasks. In addition, also the buffer management and network protocol stack processing had to be implemented. The buffer management has been implemented using a fixed number of buffers, of a fixed size, and located at fixed places in memory. This eases porting this method into a FPGA based system. The same intention was followed in the implementation of the network protocol processing, which is operating with the data in the buffers. It is implemented as a FSM, which will reduce the redesign effort of porting it to a FPGA based systems. The two related projects currently in development are described in Section 5.4.1. Both will profit from the experience gained in the development of the GE-MN.

The measured technical key data of the network part of the GE-MN are a round trip latency of $(57 \pm 9) \mu \mathrm{s}$ and a maximum full duplex throughput of $20 \mathrm{MB} / \mathrm{s}$. The round trip latency time is significantly better than in a typical personal computer (especially in jitter) and the limitation in throughput is discussed in Section 3.2.

The development of the GE-MN has shown that the development of a hardware platform, which uses Gigabit-Ethernet as a standard network technology for data transport, is feasible in the context of physics experiments at particle accelerators. Provided, the requirements for the network parameters latency (round trip $<60 \mu \mathrm{s}$ ) and bandwidth $(1 \mathrm{GBit})$ can be meet. In addition, it provided expertise to aid the development of the successor projects summarized shortly in the next sections. 


\subsubsection{Directly Related Projects}

Two ongoing projects in the work-group are directly related to the GE-MN: A HandleC [Cel07] implementation of a networked analysis system on a RC300 system [Kir07] and the FPGA-based Compute Nodes [Per07].

HandleC is a C-like programming language with the capability to be synthesized into configurable hardware platforms like FPGA chips. The C-like syntax should provide a more elegant programming interface to implement algorithms in FPGA chips, since C-based languages are more convenient for these task than real hardware programming languages like VHDL. The current project is to implement a similar functionality as available in the GE-MN using the HandleC language to program the commercially available RC300 board [Cel07]. The key components of the RC300 are a large FPGA and two Gigabit-Ethernet interfaces. The whole implementation, including PL3-interface programming, Ethernet packet handling, IP stack processing and data analysis application will be running in one FPGA. The comparison of this approach to the more conventional approach taken in the implementation of the GE-MN will show the benefits and challenges inherent to these new language concept. This project will provide the experience to decide how to use these new type of language in system design and programming. The project is currently in the implementation stage.

The second ongoing project is the design and implementation of a FPGA-based Compute Node shown in Figure 5.2 providing a lot of network IO capabilities and a lot of compute power via four large FPGA chips. In addition, it is based on the new ATCA system standard, which is a possible successor of the VMEbus standard. This Compute-Node is a common project for the DAQ of several experiments and developed in cooperation. The experiments are the BESIII experiment at IHEP in Beijing, the HADES experiment and the PANDA experiment (both at GSI). The foreseen role of this system in HADES is shown in Figure 5.3. A full ATCA crate of these FPGA-based Compute Nodes (up to 14 Compute Nodes) will serve as the Trigger-LinkHubs and the Central-Trigger-System. This system will perform the correlations of the different detector systems. One foreseen algorithm which really needs the highly parallel compute power of this system is the full MDC to RICH correlation. Unlike the presented method of this thesis, it will build tracks from the MDC wire chamber data in real-time, and correlate these with the RICH raw data (compare Section 4.2). Here, the raw data are not the samples provided by the RICH IPU. Instead, the raw pad plane information will be searched for ring signatures at places indicated by the online reconstructed MDC tracks.

As of this date, this project is still in an early phase. The development of circuit drawings is converging to an end, but no hardware programming has been done so far. Also not touched are the mentioned higher level trigger and correlation methods.

\subsubsection{The New HADES DAQ and Trigger System}

The HADES DAQ and trigger system is being upgraded [Tra07, Tra06b, HAD06]. Figure 5.3 shows the intended new system layout, which has a lot of advantages over the current system. Namely, it reduces the number of different sub-systems and bus-systems connecting these, and thus unifies and simplifies the whole system. It will have a higher performance, as much faster components and links are used. Further, the use of optical connections make the data transport virtually immune to external noise, and also does not introduce additional noise in the system. Both noise coupling effects have been an issue in the existing system. A central platform in the new system is the TRB (Trigger and Readout Board) shown and commented in Figure 5.3. 


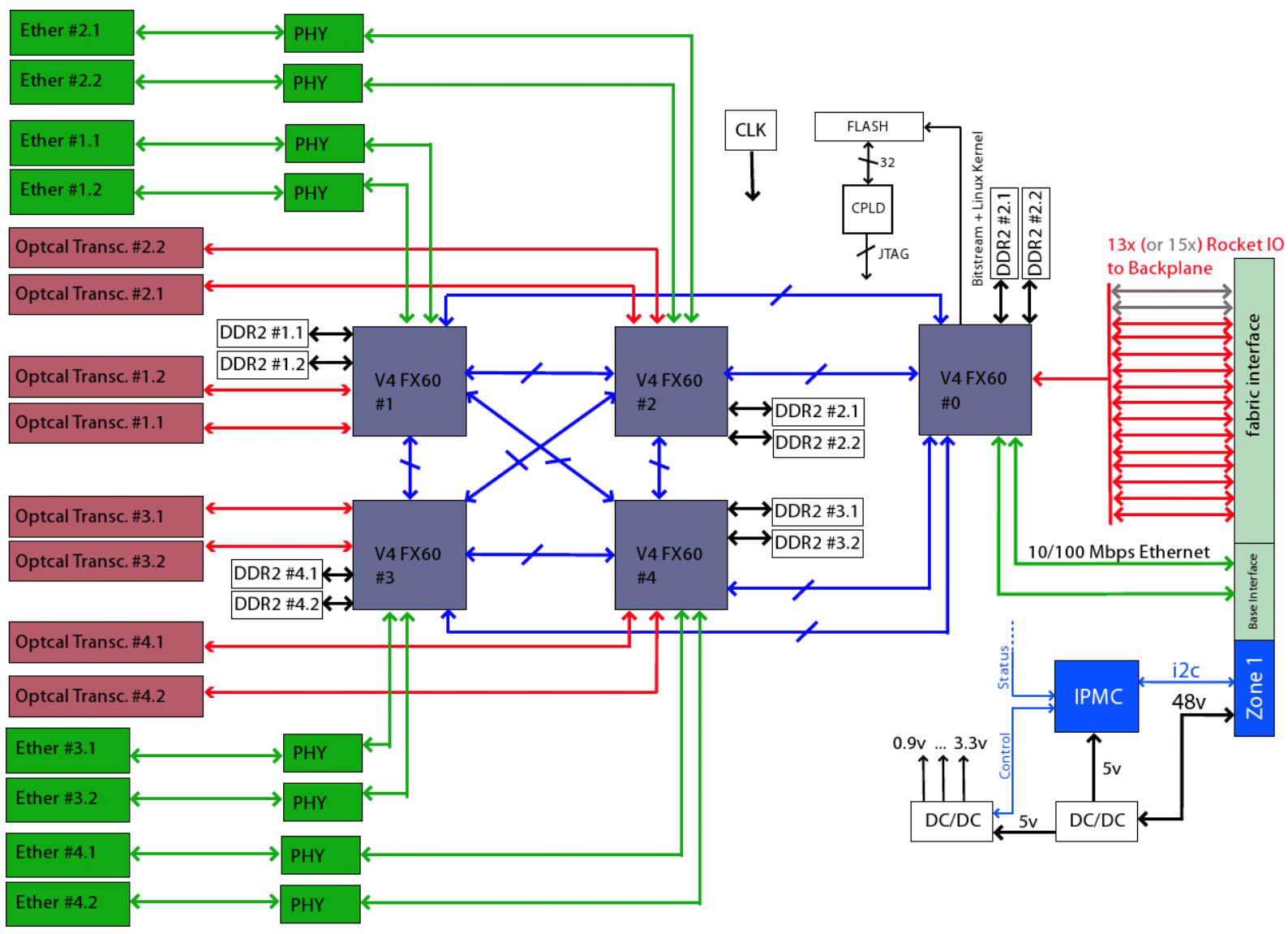

Figure 5.2.: FPGA-based Compute Node [Per07].

The FPGA-based Compute Node features 4 Virtex-4 FPGA chips with two embedded PowerPC processors and local DRAM as computing resources. FPGA number 0 is used for system management, control, communication and data flow management. Every FPGA has the option of running an embedded Linux operating system. A huge IO capability is realized via 13 RocketIO connections to the back-plane, and 4 Gigabit-Ethernet links and 8 Optical links to cable connectors for a total aggregate bandwidth of $\approx 30 \mathrm{GBit} / \mathrm{s}$. The RocketIO back-plane connections provide communication to other FPGA-based Compute Nodes in the same ATCA shelf via a full star mesh on the ATCA back-plane. 


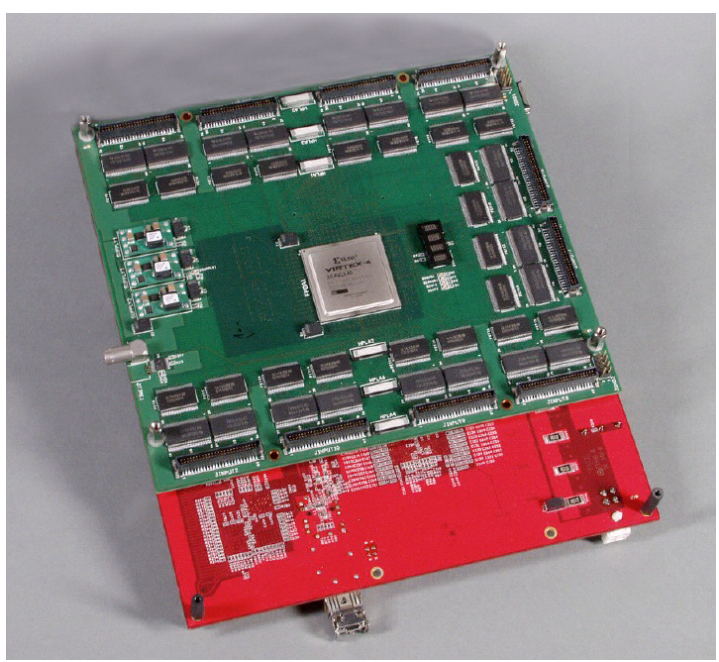

(a) MDC Readout on TRB

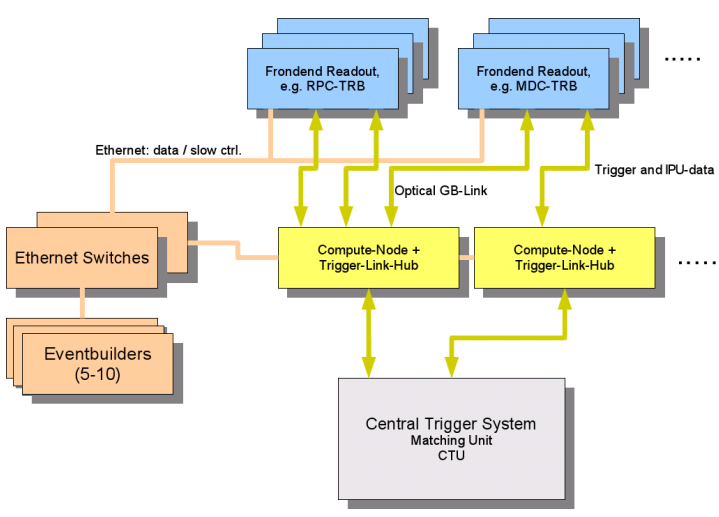

(c) new DAQ Scheme

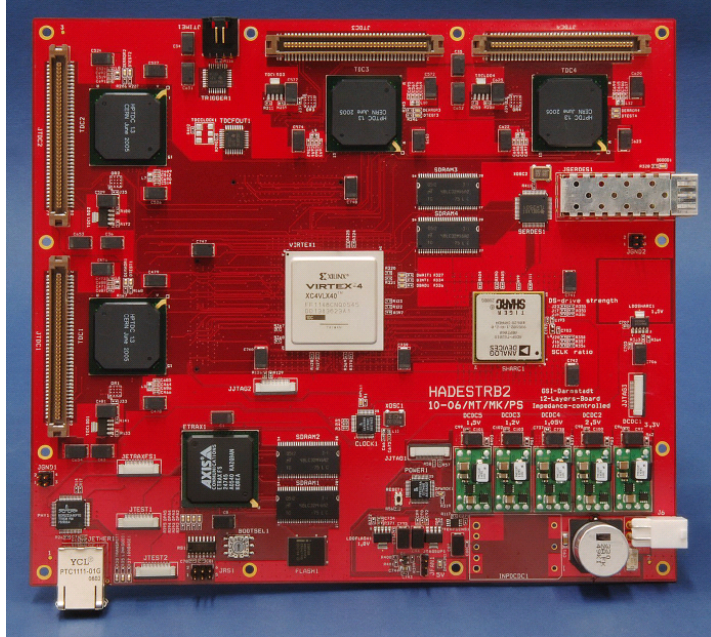

(b) TRB PCB

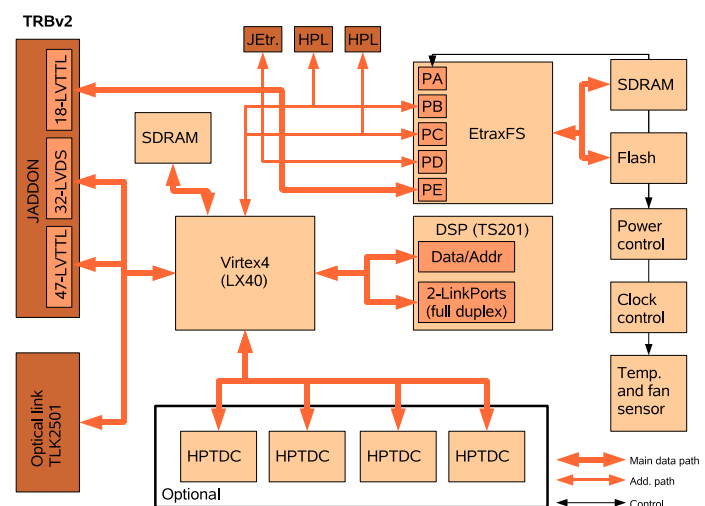

(d) TRB Block Diagram

Figure 5.3.: TRB, TRB MDC Add-on, and planned new DAQ scheme [Tra07, Sko07, Tra06b].

The picture (a) shows the TRB MDC add-on board connected to the backside of the TRB, which is shown in picture (b). The schematic of the TRB is shown in (d). The TRB features 4 times 32 TDC channels connected to a large FPGA. A TigerSHARC DSP is present for data processing. High performance, low latency data transfer is possible via the optical link. The EtraxFS[Axi07] is an embedded system. It is used for remote slow control and data transport, using its Ethernet connectivity. For the use of the TRB as a carrier of the MDC readout board, the TDC chips can be left away. The new, planned readout schema is sketched in (c) and shows how the data needed for the trigger is transferred from the front-end boards (TRB) via optical links to the Compute-Node and Trigger-Link-Hub. From there, it is transferred to the Central Trigger System where trigger decisions are taken. The data is transferred via Ethernet to the event-builder, where the data is stored to disk and tape. 


\subsubsection{RICH to MDC Correlation in the new HADES Trigger}

The readout of the TDC channels of the MDC detector is being implemented as an add-on board for the TRB, and it is shown in Figure 5.4. In the existing DAQ and trigger scheme the MDC data information is not used in the trigger and no data path exists which would permit this. This will change in the planned system, as explained in Figure 5.4. The ideal way of implementing the correlation algorithm described in Chapter 4 in the new system would be to send the RICH IPU ring data to the TRB boards and perform the correlation in each TRB for its MDC chamber. It is also conceivable to sent directly the calculated crossed cell numbers (compare Section 4.2.1) to avoid the need of calculating them in the individual TRB boards. The result of the correlation is here the number of matched layers found by the TRB for its MDC chamber. This result can be sent to the Central Trigger System (compare Figure 5.3 (c)), which only has to collect and add up the matched layers per sector to take this type of trigger decision.

Another, more flexible possibility is to deliver the correlation result in an array analogous to the one described in Section 4.4.3. This splits the method up into calculating the full correlation per MDC chamber, and to take the actual trigger decision based on this data in a central place. In this scheme, the additional load is very small. Instead of the number of matched layers (one number per MDC chamber) the sorted arrays have to be transferred ( 6 entry array per MDC chamber). A little additional load is generated in the central system, because the two arrays per sector have to be combined into a 12 entry, sorted array. The trigger decision becomes then just the Boolean expression deltaMinSortSectorLay12 $\left[L_{M}\right]<M_{W}$ for each sector.

Referring to the performance discussion in Section 5.3 the theoretical performance of the RICH to MDC correlation in the TRB based system can be estimated. The DSP of the TRB is two times faster than the DSP of the GE-MN, and the algorithm is split up for six sectors times two modules. The result is a performance estimate of 1400 events $/ \mathrm{s} \times 2 \times 6 \times 2=33,6 \mathrm{kHz}$ for $\mathrm{Ar}+\mathrm{KCl}$ data, where the lowest performance of the algorithm has been measured. It assumes that the involved system has a capacity utilization of $100 \%$ and excludes any latency (waiting for data) and additional protocol overhead effects. Still, an assumption of $\approx 30 \mathrm{kHz}$ can be treated as realistic, since the performance estimate is derived from the GE-MN. The GE-MN has to handle the data transfer via UDP/IP on Ethernet, which is significantly more involved (due to the protocol stack involved; see Section 3.2) than the direct data readout of the wire chamber data by the TRB MDC add-on modules. In addition, the compute power of the two FPGA chips (TRB and add-on) is not at all taken into account.

An other possible setup is to perform the correlation completely in a central place. When the Compute-Node described in Section 5.4.1 is available as central trigger system, as described in Figure 5.4 (c), the correlation can also be performed there. Which option is more appropriate to the problem depends on two factors. First, it has to be considered if the the performance of the implementation described above is sufficient. If not, an implementation in the 14 Compute Nodes promises to have a much higher performance. If this option has to be taken a complete reimplementation of the correlation method in the FPGA chips has to be done. This is an involved procedure since the RICH to MDC correlation uses quite a lot of floating point arithmetic and several trigonometric functions (compare Chapter 4). This functionality is not easily available in FPGA chips, in contrast to the classical digital signal processors used in the TRB systems. 


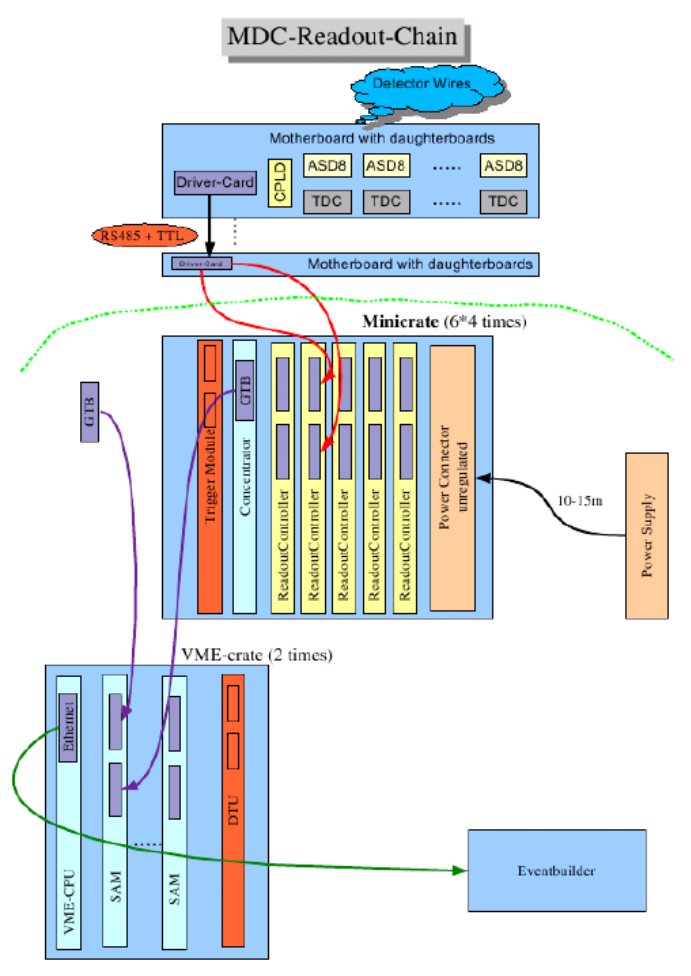

(a) Curent System

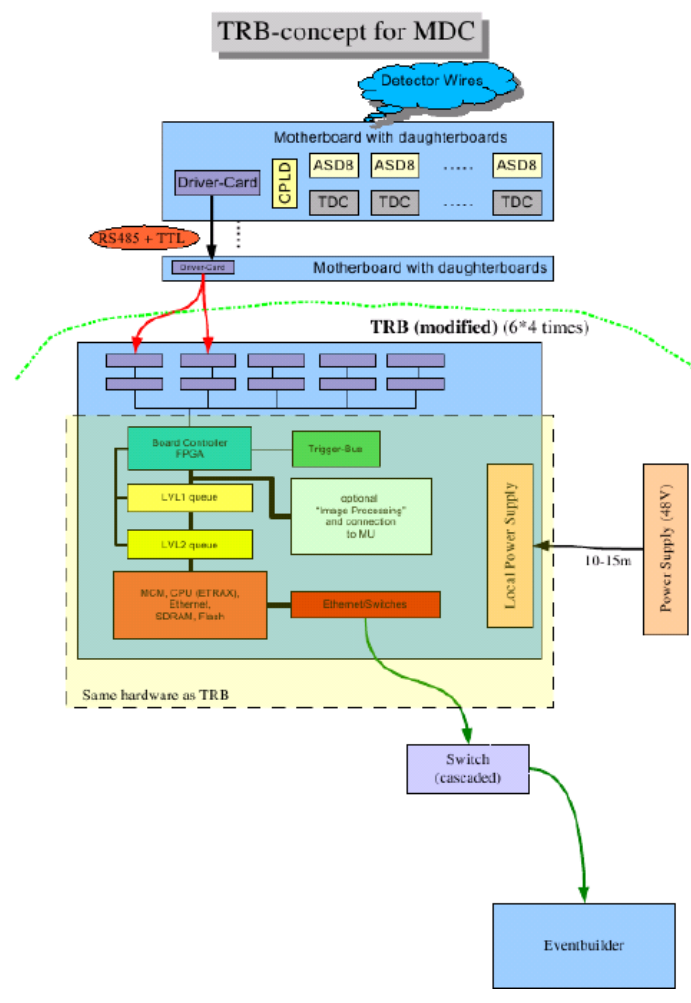

(b) Planned System

Figure 5.4.: MDC Readout Schemata: Old Readout versus planned New Readout [Tra07].

Figure (a) shows the existing read-out system of the HADES DAQ while figure (b) shows the planned extension and unification. The two layers of data buffering and concentration, the ROC ( Read Out Controller) and the SAM (German: Steuerung und Auslese Modul; Read-Out and Control Module), are exchanged with the TRB and the TRB MDC add-on. The new system uses fewer components (only one TRB and add-on board per MDC module) than the existing system. In addition, it is important to note that the MDC data in the new system is transported via the switch (Trigger-Link-Hub in Figure 5.3 (c)) of the trigger and DAQ system. This allows the use of the MDC data in the trigger system. Further, the use of the TRB boards provides a DSP and a FPGA for the local processing of the data per MDC module. 


\subsection{Bibliography}

[Axi07] AxIs; The Axis Website (2007); URL http://www.axis.com/products/dev/index. $\mathrm{htm}$

[Cel07] Celoxica; The Celoxica Website (September 2007); URL http://www.celoxica.com

[HAD06] HADES Collaboration; The HADES Wiki (2006); URL http://hades-wiki.gsi. de

[Kir07] Camilla KiRChHüBEL; private communication (September 2007)

[Per07] Tiago Perez; Ming Liu et al.; private communication (September 2007)

[Sko07] P. SkotT et al.; A General Purpose Trigger and Readout Board for HADES and FAIRExperiments (May 2007); Presented at 15th IEEE Real Time Conference 2007 (RT 07), Batavia, Illinois, 29 Apr - 4 May 2007.

[Toi04] AlBerica Toia (HADES); Performance of the HADES Spectrometer for Dilepton Identification in the Reaction $C+C$ at $1 \mathrm{AGeV}$; Ph.D. thesis; II. Physikalisches Institut der Justus-Liebig-Universität Gießen (March 2004); URL http://pcweb. physik. uni-giessen.de/exp2

[Tra01] Michael Traxler (HADES); Real-Time Dilepton Selection for the HADES Spectrometer; Ph.D. thesis; II. Physikalisches Institut der Justus-Liebig-Universität Gießen (2001); URL http://pcweb.physik.uni-giessen.de/exp2

[Tra06b] Michael Traxler; Ingo Fröhlich; M. Kajetanowicz et al.; A General Purpose Trigger and Readout Board (TRB) for HADES and FAIR-Experiments; Technical Report; GSI (2006); GSI Scientific Report; URL http://www.gsi.de/informationen/ wti/library/scientificreport2006

[Tra07] Michael Traxler; Ingo Fröhlich; Attilio Tarantola et al.; TRB Publication List in HADES Wiki; Technical Report; GSI (July 2007); URL http://hadeswiki.gsi.de/cgi-bin/view/DaqSlowControl/TRBPublicationList 


\section{A. Glossary}

This chapter is a short summary of abbreviation, acronyms and terms which are usually not common knowledge, but which are helpful to be known in the context of this work.

ARP $\underline{\text { Address }} \underline{\text { Resolution }}$ Protocol — Network protocol mapping IP addresses to other addressing systems.

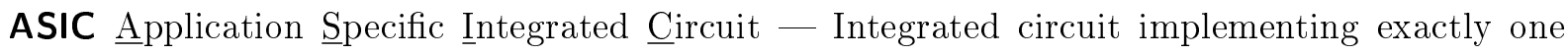
predefined functionality. For example a DSP, a SRAM or an ADC.

Bit Smallest unit in information theory with two states (binary), visualized usually as '0' (or: off/low) and '1' (or: on/high).

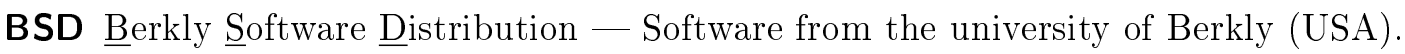

Byte Nowadays usually defined to be 8 Bit.

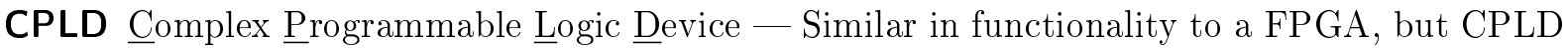
architectures feature a big product term matrix and the configuration is typically persistent (FLASH).

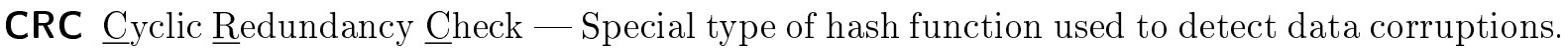

(Data) Word Architecture dependent unit containing a certain number of bits, e.g. a 32 bit DSP uses a standard word size of 4 Bytes $=32$ Bits.

DC/DC DC to DC voltage converter circuit, i.g. $5 \mathrm{~V}$ to $3,3 \mathrm{~V}$.

DDR Double Data Rate — Transfers data on rising and falling clock edges and thus doubles the data rate per clock cycle.

DMA Direct $\underline{\text { Memory }}$ Access - Memory access from/to a memory managed by DMA controller and performed without intervention of the host processor.

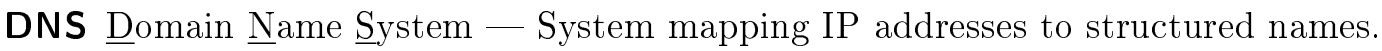

DSP Digital Signal Processor — Processor optimized for signal processing in embedded solutions.

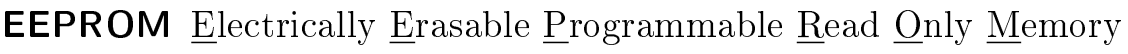

Ethernet Most widely used LAN technology.

FCS Frame $\underline{\text { Check }}$ Sequence - The 32 Bit CRC of an Ethernet frame.

Firmware Low level software running in an embedded system, usually not user serviceable and persistent. 
FLASH Special EEPROM in which memory pages can be "flashed", meaning erased and reprogrammed.

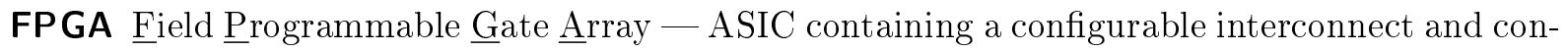
figurable logic elements, such as combinatorials (AND/OR/NOT,...), registers (Flip-Flops), possibly clock synthesizers (PLL based) and even DSP elements (Adder/Multiplier/Divider). Modern FPGA architectures are typically SRAM LUT based and thus the configuration is volatile.

FSM Finite $\underline{\text { State }}$ Machine - System that can be only in one of its predefined states. State transitions are triggered by well defined events.

GB/GByte Giga Byte $-1 \mathrm{~GB}=1024 \mathrm{MB}$. Compare also MB.

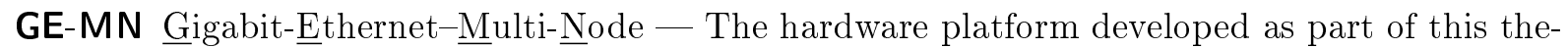
sis.

Harvard Architecture Processor architecture with separated paths for instructions and data.

HDL Hardware Description Language - Special language used to design, simulate and synthesize (primarily) digital circuits. HDL synthesis is the process of inferring the logic elements of a certain logic family to generate a circuit with a behavior which is clock cycle wise identical to the description in the HDL source code.

ICMP Internet Control and Message Protocol — Status and control message protocol of the TCP/IP family.

IDE Integrated Development Environment - Software package containing compiler, assembler, disassembler and emulator in a graphical user interface.

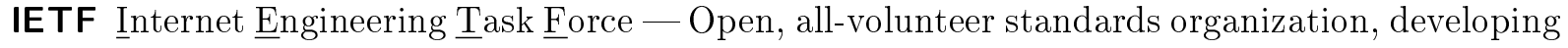
the primarily Internet protocol standards.

IP Internet Protocol - Inter-networking layer protocol of the TCP/IP family.

IPU Image Processing $\underline{\text { Unit }}$ - HADES lingo for cards recognizing certain patterns (image) produced by the detectors.

IRQ Interrupt Request — Signal of a special event which needs processing outside the standard program flow.

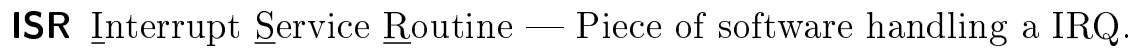

JTAG Joint Test $\underline{\text { Access }}$ Group - IEEE Standard 1149.1 used as a vendor independent bus to access chips in a chain. Commonly used to download programs into FPGA and CPLD chips.

KB/KByte One kilo Byte in information technology is equal to $2^{10}$ byte $=1024$ byte $=1 \mathrm{~KB}$ unlike the usual kilo (k) symbol.

LAN Local Area Network - Network technology for short range communication. Typically maximum network segments length are in the order of a few hundred meters. 
LUT Look Up Table - A LUT stores precalculated computation results for direct retrieval instead of run-time calculation.

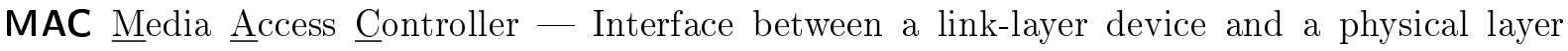
implementation. This chip implements the Ethernet protocol.

Magnetics DC decoupling and analog NEXT suppressor circuit used in LAN, WAN and telephone systems.

MB/MByte Mega Byte $-1 \mathrm{MB}=1024 \mathrm{~KB}$. Compare also KB.

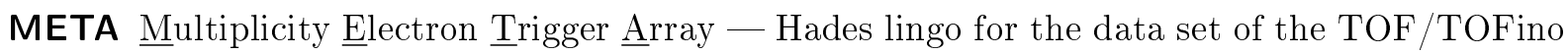
and PreShower detector

MTU $\underline{\text { Maximum }}$ Transfer $\underline{\text { Unit }}$ - Maximum size of a transfer unit on a network transporting IP traffic.

NEXT Near End cross $(\underline{X})-\underline{T}$ alk - Crosstalk induced by a sender on its very own receive inputs. Needs to be canceled or rejected in many applications, especially those with full duplex capability on the same wire (pair). Classical example: analog telephone with only two wires for full duplex, arbitration(ring) and power.

NIC Network Interface Card - Circuit connected to a back-plane bus providing network connectivity for the system.

Octet Unit used to measure the size of transported data in network communication. One Octet is always eight bits (unlike a byte).

OS Operating System - Software layer managing hardware and software below the applications.

PCI Peripheral Component Interconnect - A standard back-plane bus architecture. Uses synchronous transfers at typically $33 \mathrm{MHz}$ and 32 Bit bus width. Address and data lines are shared.

PHY/PHYter Physical layer access controller — Interface to the physical medium, i.e copper wires, fiber optic cables or even homing pigeons.

ping Software tool to measure latency as RTT in TCP/IP based networks. Available in most operating systems.

PLL Phase Locked Loop - Circuit generating a signal which is locked to frequency and phase of a input signal.

POS-PHY Layer 3 (Multi-)point to (multi-)point bus system used predominantly in the telecommunications industry to interface data stream from/to WAN and LAN networks.

PRC $\underline{\text { Pattern }} \underline{\text { Recognition }}$ Card - HADES lingo for cards recognizing certain patterns produced by the detectors.

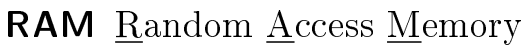

real-time A real-time system guaranties to perform its tasks in a predefined time frame. 
RFC Request For Comments - A RFC is a IETF open and public standard.

RICH Ring Imaging Cherenkov (Detector) - Detector focusing the Cherenkov light of charged particles with a mirror as a ring shape onto a readout plane.

RJ-45 Connector standard popular in wire based LAN technologies.

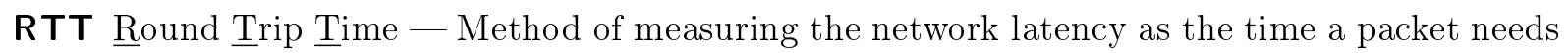
to travel to a destination and back again to the sender.

SFD $\underline{\text { Start }}$ of Frame Delimiter - Special bit sequence at the beginning of an Ethernet frame. Makes frame detection more easy.

SRAM Synchronous RAM - Register (Flip-Flop) based RAM.

STD Internet Standard - Collection of RFC documents describing the essential Internet protocol standards.

TCP/IP protocol Suite of layered protocols consisting of ARP, IP, ICMP, UDP, TCP. Often additional protocols are also implicitly included when speaking about TCP/IP, e.g. DNS.

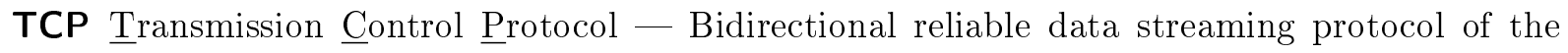
TCP/IP family.

TDC $\underline{\text { Time to }}$ Digital Converter - Circuit converting the time information of an analog signal to digital data.

TigerSHARC One specific DSP from the company Analog Devices based on an extended Harvard architecture.

TOF Time of $\underline{\text { Flight } ~-~ D e t e c t o r ~ m e a s u r i n g ~ t h e ~ f l i g h t ~ t i m e ~ o f ~ p a r t i c l e s . ~}$

UDP $\underline{\text { User }}$ Datagram Protocol - Unidirectional unreliable packet transmission protocol of the TCP/IP family.

VHDL VHSIC Hardware Description Language - A widely used and standardized HDL with ADA like syntax.

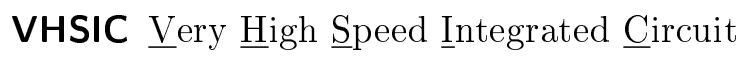

VMEbus Expandable back-plane bus architecture derived from the bus of the Motorola $68 \mathrm{~K}$ processor family.

WAN Wide Area Network - Network technology for long range communication in contrast to LAN technologies. 


\section{B. HADES Collaboration Authors List}

The HADES collaboration as of January 2007.

\section{The HADES Collaboration}

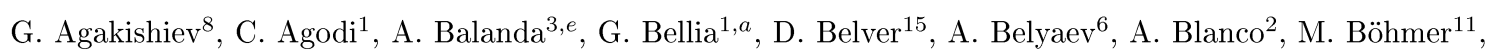
J. L. Boyard ${ }^{13}$, P. Braun-Munzinger ${ }^{4}$, P. Cabanelas ${ }^{15}$, E. Castro ${ }^{15}$, S. Chernenko ${ }^{6}$, T. Christ ${ }^{11}$, M. Destefanis ${ }^{8}$, J. Díaz ${ }^{16}$, F. Dohrmann ${ }^{5}$, A. Dybczak ${ }^{3}$, T. Eberl ${ }^{11}$, L. Fabbietti ${ }^{11}$, O. Fateev ${ }^{6}$, P. Finocchiaro ${ }^{1}$, P. Fonte ${ }^{2, b}$,

J. Friese ${ }^{11}$, I. Fröhlich ${ }^{7}$, T. Galatyuk ${ }^{4}$, J. A. Garzón ${ }^{15}$, R. Gernhäuser ${ }^{11}$, C. Gilardi ${ }^{8}$, M. Golubeva ${ }^{10}$, D. González-Díaz ${ }^{4}$, E. Grosse ${ }^{5, c}$, F. Guber ${ }^{10}$, M. Heilmann ${ }^{7}$, T. Hennino ${ }^{13}$, R. Holzmann ${ }^{4}$, A. Ierusalimov ${ }^{6}$, I. Iori $^{9, d}$, A. Ivashkin ${ }^{10}$, M. Jurkovic ${ }^{11}$, B. Kämpfer ${ }^{5}$, K. Kanaki ${ }^{5}$, T. Karavicheva ${ }^{10}$, D. Kirschner ${ }^{8}$, I. Koenig ${ }^{4}$, W. Koenig ${ }^{4}$,

B. W. Kolb ${ }^{4}$, R. Kotte ${ }^{5}$, A. Kozuch ${ }^{3, e}$, F. Krizek ${ }^{14}$, R. Krücken ${ }^{11}$, W. Kühn ${ }^{8}$, A. Kugler ${ }^{14}$, A. Kurepin ${ }^{10}$,

J. Lamas-Valverde ${ }^{15}$, S. Lang ${ }^{4}$, J. S. Lange ${ }^{8}$, L. Lopes ${ }^{2}$, L. Maier ${ }^{11}$, A. Mangiarotti ${ }^{2}$, J. Marín ${ }^{15}$, J. Markert ${ }^{7}$,

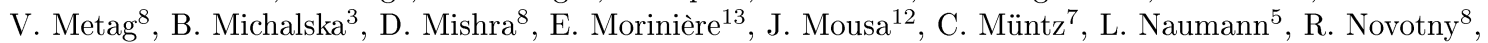

J. Otwinowski ${ }^{3}$, Y. C. Pachmayer ${ }^{7}$, M. Palka ${ }^{4}$, Y. Parpottas ${ }^{12}$, V. Pechenov ${ }^{8}$, O. Pechenova ${ }^{8}$, T. Pérez Cavalcanti ${ }^{8}$, J. Pietraszko ${ }^{4}$, W. Przygoda ${ }^{3, e}$, B. Ramstein ${ }^{13}$, A. Reshetin ${ }^{10}$, M. Roy-Stephan ${ }^{13}$, A. Rustamov ${ }^{4}$, A. Sadovsky ${ }^{10}$, B. Sailer ${ }^{11}$, P. Salabura ${ }^{3}$, A. Schmah ${ }^{4}$, R. Simon ${ }^{4}$, S. Spataro ${ }^{8}$, B. Spruck ${ }^{8}$, H. Ströbele ${ }^{7}$, J. Stroth ${ }^{7,4}$, C. Sturm ${ }^{7}$,

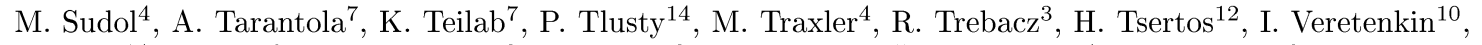

V. Wagner ${ }^{14}$, H. Wen ${ }^{8}$, M. Wisniowski ${ }^{3}$, T. Wojcik ${ }^{3}$, J. Wüstenfeld ${ }^{5}$, S. Yurevich $^{4}$, Y. Zanevsky ${ }^{6}$, P. Zumbruch $^{4}$ (HADES collaboration)

${ }^{1}$ Istituto Nazionale di Fisica Nucleare - Laboratori Nazionali del Sud, 95125 Catania, Italy

${ }^{2}$ LIP-Laboratório de Instrumentação e Física Experimental de Partículas , 3004-516 Coimbra, Portugal

${ }^{3}$ Smoluchowski Institute of Physics, Jagiellonian University of Cracow, 30-059 Kraków, Poland

${ }^{4}$ Gesellschaft für Schwerionenforschung mbH, 64291 Darmstadt, Germany

${ }^{5}$ Institut für Strahlenphysik, Forschungszentrum Dresden-Rossendorf, 01314 Dresden, Germany

${ }^{6}$ Joint Institute of Nuclear Research, 141980 Dubna, Russia

${ }^{7}$ Institut für Kernphysik, Johann Wolfgang Goethe-Universität, 60438 Frankfurt, Germany

${ }^{8}$ II.Physikalisches Institut, Justus Liebig Universität Giessen, 35392 Giessen, Germany

${ }^{9}$ Istituto Nazionale di Fisica Nucleare, Sezione di Milano, 20133 Milano, Italy

${ }^{10}$ Institute for Nuclear Research, Russian Academy of Science, 117312 Moscow, Russia

${ }^{11}$ Physik Department E12, Technische Universität München, 85748 München, Germany

${ }^{12}$ Department of Physics, University of Cyprus, 1678 Nicosia, Cyprus

${ }^{13}$ Institut de Physique Nucléaire d'Orsay, CNRS/IN2P3, 91406 Orsay Cedex, France

${ }^{14}$ Nuclear Physics Institute, Academy of Sciences of Czech Republic, 25068 Rez, Czech Republic

${ }^{15}$ Departamento de Física de Partículas, University of Santiago de Compostela, 15782 Santiago de Compostela, Spain

${ }^{16}$ Instituto de Física Corpuscular, Universidad de Valencia-CSIC, 46971 Valencia, Spain

${ }^{a}$ also at Dipartimento di Fisica e Astronomia, Università di Catania, 95125 Catania, Italy

${ }^{b}$ also at ISEC Coimbra, Coimbra, Portugal

${ }^{c}$ also at Technische Universität Dresden, 01062 Dresden, Germany

${ }^{d}$ also at Dipartimento di Fisica, Università di Milano, 20133 Milano, Italy

e also at Panstwowa Wyzsza Szkola Zawodowa, 33-300 Nowy Sacz, Poland 


\section{List of Figures}

1.1. Strong Coupling "Constant" $\alpha_{\mathrm{S}}(Q)$ by Momentum Transfer [Tas01] . . . . . . . . 16

1.2. Schematic Picture of the Color Field and the String Breaking. . . . . . . . . . . 17

1.3. Normalized Quenched Wilson Action SU(3) Potential [Bal00]. . . . . . . . . . . 17

1.4. Mexican hat potential in 3D [Koc02] . . . . . . . . . . . . . . 18

1.5. Linear Sigma Model Mexican Hat Potential as 2D cut [Koc02]. . . . . . . . . . . 20

1.6. Dependency of the Quark Condensate on Temperature and Density [Kli90]. . . . 21

1.7. QCD Phase Diagram [Gut06] . . . . . . . . . . . . . . . . . . 22

1.8. Feynman Diagrams of Electron Scattering and Annihilation. . . . . . . . . . 24

1.9. Pion Form Factor (time-like) [Kli96]. . . . . . . . . . . . . . . . . . 26

1.10. Coupling of Virtual Photons to Hadrons in the VMD Model. . . . . . . . . . . . 26

1.11. Recent HADES Dilepton $\left(e^{+} e^{-}\right)$Spectra from C + C [Aga07]. . . . . . . . . . . 28

1.12. NA60 Dilepton $\left(\mu^{+} \mu^{-}\right)$Spectra in In+In Collisions [Arn06] . . . . . . . . . . . 29

2.1. Artist's view of the HADES spectrometer $[$ Sch06] . . . . . . . . . . . . 36

2.2. Schematic of the HADES spectrometer shown in a lateral cut [HAD94]. . . . . . 37

2.3. The START/VETO detectors. . . . . . . . . . . . . . . . . . . . 39

2.4. Schematic lateral cut of the RICH detector of HADES [Böh99]. . . . . . . . . . . 42

2.5. Optical Parameters of the HADES RICH components [Fab03a, Fab03b, Leh00]. . 43

2.6. Ring Recognition Search Mask and Pad Groups [Leh00, Leh03] . . . . . . . . . . 44

2.7. Wire layout in the MDC modules. . . . . . . . . . . . . . . . . 45

2.8. MDC Drift Cell Characteristics [Mar05]. . . . . . . . . . . . . . . . . 48

2.9. The Pre-Shower Detector and TOFino Detector [Bal04] . . . . . . . . . . . 51

2.10. Inner Projection Planes [Aga01, Rus06]. . . . . . . . . . . . . . . . 53

2.11. Position of the Kick-Plane and Projection Planes [Aga01, Rus06] . . . . . . . . . 54

2.12. Track Candidate Search (cluster finder) [Aga01, Rus06] . . . . . . . . . . . . 55

2.13. The HADES Trigger System [Leh99]. . . . . . . . . . . . . . . . . . 56

3.1. The GE-MN System Architecture. . . . . . . . . . . . . . . . . . . . . . . 64

3.2. The GE-MN Printed Circuit Board (Top Side). . . . . . . . . . . . . . . . 65

3.3. GE-MN Network Communication Model (UDP/IP Network Stack). . . . . . . . . 71

3.4. Network Topology: Ring and Star . . . . . . . . . . . . . . . . . . . . . 73

3.5. Receive Network Buffer Management in the GE-MN. . . . . . . . . . . . . . . . 74

3.6. Typical PC Network Latency (Gigabit Ethernet). . . . . . . . . . . . . . . . . . 76

3.7. Example "ping" Measurements of the GE-MN. . . . . . . . . . . . . . . . . 77

4.1. Definition Good Leptons for the Real Data. . . . . . . . . . . . . . . . . . . 83

4.2. HADES Geometry for the Crossed Cell Calculation (calcXed). . . . . . . . . . 87

4.3. Triangle for the last Step in the Calculation of the Crossed Cell. . . . . . . . . . 88 
4.4. Difference between calcXed() (GE-MN) and calcCrossedCells() (HYDRA) . . . . 90

4.5. $\mathrm{p}+\mathrm{p} @ 2,2 \mathrm{GeV}$ : RICH Ring Distribution from IPU Data (available online). . . . . 95

4.6. $\mathrm{p}+\mathrm{p} @ 2,2 \mathrm{GeV}$ : RICH IPU Ring to MDC Cell Correlation (available online). . . 96

4.7. $\mathrm{p}+\mathrm{p} @ 2,2 \mathrm{GeV}$ : RICH IPU Ring to MDC Cell Correlation for good Leptons. . . 97

4.8. $\mathrm{p}+\mathrm{p} @ 2,2 \mathrm{GeV}$ : Matched Layers Distributions (available online). . . . . . . . . . 98

4.9. $\mathrm{p}+\mathrm{p} @ 2,2 \mathrm{GeV}$ : Matched Layers Distributions for good Lepton Events. . . . . . . . 99

4.10. p + p@2,2 GeV: Good Lepton Base Data. . . . . . . . . . . . . . . . . . . . . . . 101

4.11. p + p@2,2 GeV: $\vartheta$ - Efficiency Distributions . . . . . . . . . . . . . . . . . . . . 102

4.12. $\mathrm{p}+\mathrm{p} @ 2,2 \mathrm{GeV}: \phi$ - Efficiency Distributions . . . . . . . . . . . . . . . . . 103

4.13. $\mathrm{p}+\mathrm{p} @ 2,2 \mathrm{GeV}:$ Momentum Efficiency Distributions . . . . . . . . . . . . . . . . 104

4.14. C+C@1,0 AGeV: RICH Ring Distribution from the RICH IPU (available online). 105

4.15. C+C@1,0 AGeV: RICH IPU to MDC Cell Correlation first Run (available online). 106

4.16. C+C@1,0 AGeV: Matched Layers Distributions first Run (available online). . . . 107

4.17. C+C@1,0 AGeV: RICH IPU Ring to MDC Cell Correlation (available online). . . 108

4.18. C+C@1,0 AGeV: RICH IPU Ring to MDC Cell Correlation for good Leptons. . 109

4.19. C+C@1,0 AGeV: Matched Layers Distributions (available online). . . . . . . . . . 110

4.20. C+C@1,0 AGeV: Matched Layers Distributions for good Lepton Events. . . . . . 111

4.21. C+C@1,0 AGeV: Good Lepton Base Data. . . . . . . . . . . . . . . . . . . . . . . 113

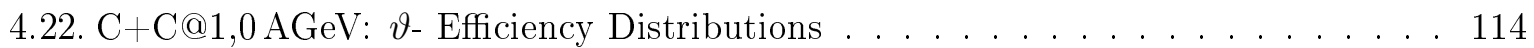

4.23. C+C@1,0 AGeV: $\phi$ - Efficiency Distributions . . . . . . . . . . . . . . . . . 115

4.24. C+C@1,0 AGeV: Momentum Efficiency Distributions . . . . . . . . . . . . . . . 116

4.25. Ar+KCl@1,756 AGeV: RICH Ring Distribution from RICH IPU (available online). 117

4.26. Ar+KCl@1,756 AGeV: RICH IPU Ring to MDC Cell Correlation (available online).118

4.27. Ar+KCl@1,756 AGeV: RICH IPU Ring to MDC Cell Correlation good Leptons. 119

4.28. Ar+KCl@1,756 AGeV: Matched Layers Distributions (available online). . . . . . . 120

4.29. Ar+KCl@1,756 AGeV: Matched Layers Distributions for good Lepton Events. . . 121

4.30. Ar+KCl@1,756 AGeV: Good Lepton Base Data. . . . . . . . . . . . . . . . . . . . 123

4.31. Ar+KCl@1,756 AGeV: $\vartheta$ - Efficiency Distributions . . . . . . . . . . . . . . . . . 124

4.32. Ar+KCl@1,756 AGeV: $\phi$ - Efficiency Distributions . . . . . . . . . . . . . . 125

4.33. Ar+KCl@1,756 AGeV: Momentum Efficiency Distributions . . . . . . . . . . . . 126

5.1. Number of Correlations Performed between RICH IPU Rings and MDC Cells. . 132

5.2. FPGA-based Compute Node [Per07]. . . . . . . . . . . . . . . . . . 137

5.3. TRB, TRB MDC Add-on, and planned new DAQ scheme [Tra07, Sko07, Tra06b]. 138

5.4. MDC Readout Schemata: Old Readout versus planned New Readout [Tra07]. . . 140 


\section{List of Tables}

1.1. Quark Properties Summary [Yao06a]. . . . . . . . . . . . . . . . . . . 15

1.2. Selected Light Mesons $[\mathrm{Yao} 06 \mathrm{a}] \ldots \ldots \ldots \ldots$. . . . . . . . . . . . 27

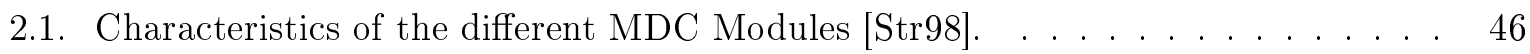

2.2. Properties of the MDC-wires [Mün04]. . . . . . . . . . . . . . . . 46

2.3. Average Detector Occupancy and Average Raw Data per Event. . . . . . . . . . 58

3.1. GE-MN memory map as seen from the VME-CPU via the VMEbus. . . . . . . . 67

4.1. Mapping Table, Online to Offline. . . . . . . . . . . . . . . . . . . 85

4.2. Trigger Decision Types. . . . . . . . . . . . . . . . . . . . 92

4.3. $\mathrm{p}+\mathrm{p} @ 2,2 \mathrm{GeV}$ : Efficiency and Enhancement for different Correlation Parameters. 100

4.4. $\mathrm{C}+\mathrm{C} @ 1,0 \mathrm{AGeV}$ : Efficiency and Enhancement for different Correlation Parameters.112

4.5. $\mathrm{Ar}+\mathrm{KCl} @ 1,756 \mathrm{AGeV}$ : Efficiency and Enhancement by Correlation Parameter. . 122 


\section{Acknowledgments}

Finally, I wish to express my gratitude to many people, without whom this thesis would not have been possible at all.

My first thanks goes to Prof. Dr. Wolfgang Kühn. His support made it possible to work on the interesting topic of this thesis in the international environment of a big collaboration. In this context, the many visits to foreign countries were especially interesting.

My parents (Hildegard Maria Klimaschka and Dietmar Reimund Kirschner ${ }^{\dagger}$ ) have my thanks for their continuous support during my years of study and their believe in by abilities during my time in school.

My gratitude goes also to many colleagues. Vladimir Pechenov, Stefano Spataro and Geydar Agakishiev for the interesting discussions and helpful suggestions directly related to the analysis part of my work.

Thanks to many more people from the work-group and from the institute, who provided a very nice and friendly working and research environment. Among them Marco Destefanis, Björn Spruck, Daniel Schäfer, Michael Traxler, James Ritman, Alberica Toia, Ingo Fröhlich, Jörg Lehnert, Ming Liu, Shuo Yang, Tiago Perez, Olga Pechenova, Zoltán Gagyi-Pálfy, Jens Sören Lange, Johannes Lang and Johannes Roskoss. Many thanks to Camilla Kirchhübel. Her Italian cooking skills, and eagerness to share them and the products, are unmatched in the institute.

The people who provided non scientific assistance should also be mentioned for their support in all the underestimated, time consuming organizational issues. Their names are Christa Momberger, Anita Rühl, Jürgen Schneider, Werner Döring, Thomas Köster, and all other unmentioned workers is the institute.

Special thanks to all the people who took the burden to hunt for all the funny spelling and grammar errors!

All people from the HADES collaboration, the Gesellschaft für Schwerionenforschung (GSI), the PANDA collaboration, and many different open source projects get my gratitude collectively, because their number is vast and I can not remember, let alone mention, then individually.

This work was supported by several grants of the BMBF: 06GI179, 06GI180, 06GI144 and 06GI145.

The final thanks goes to my girlfriend Cathrin Mucha who provided her full support and a lot of encouragement in all situations of life. 



\section{Erklärung}

Hiermit erkläre ich, daß diese Arbeit von mir selbstständig erstellt wurde und ich keine anderen als die angegebenen Hilfsmittel und Quellen verwendet habe.

Daniel Georg Kirschner 



\section{Extended and Ordered Bibliography}

Each individual chapter features a bibliography for the material referenced there. This Appendix gives is a listing of useful references sorted by topic and also includes material which has not been cited, but which has been found to be useful in the context of this thesis. The naming scheme used here is consistent with the naming scheme in the individual chapters.

\section{HADES Bibliography}

[Aga00] G. N. Agakichiev; V. N. Pechenov; Yu. V. Zanevsky et al. (HADES); Fast method for searching tracks in Multiwire Drift Chambers of HADES spectrometer (April 2000); internal HADES manual

[Aga01] G. N. Agakichiev and V. N. Pechenov (HADES); Dubna tracks reconstruction user manual (December 2001); HADES internal manual

[Aga06a] G. N. AgAKICHIEv (HADES); private communication (November 2006)

[Aga06b] G. N. Agakichiev and V. N. Pechenov (HADES); private communication (November 2006)

[Aga07] G. N. Agakichiev; C. Agodi; H. Alvarez-Pol et al. (HADES); Dielectron Production in ${ }^{12} \mathrm{C}+{ }^{12} \mathrm{C}$ Collisions at $2 \mathrm{AGeV}$ with the HADES Spectrometer; Physical Review Letters (2007); 98(5):052302; doi:10.1103/PhysRevLett.98.052302; arXiv: nucl-ex/0608031

[Ago02] C. Agodi; A. Bassi; R. BAssini et al. (HADES); The HADES time-of-flight wall; Nuclear Instruments and Methods in Physics Research A (2002); 492:14-25

[Alv04] H. Alvarez Pol; R. Alves; A. Blanco et al. (HADES); A large area timing RPC prototype for ion collisions in the HADES spectrometer; Nuclear Instruments and Methods in Physics Research A (2004); 535:277-282; doi:10.1016/j.nima.2004.07.276

[Axi07] AxIs; The Axis Website (2007); URL http://www.axis.com/products/dev/index. $\mathrm{htm}$

[Bal04] A. BALANDA et al. (HADES); The HADES Pre-Shower detector; Nuclear Instruments and Methods in Physics Research A (2004); 531:445-458

[Bel06] D. BELver et al. (HADES); The Front-End Electronics for the HADES RPC Wall (ESTRELA-FEE); Nuclear Physics B (Proc. Suppl.) (2006); 158:47-51 
[Ber99] E. Berdermann; K. Blasche; P. Moritz et al. (HADES); First Applications of CVD-Diamond Detectors in Heavy-ion Experiments; Nuclear Physics B (Proc. Suppl.) (1999); 78:533-539

[Bla03] A. Blanco; R. Ferreira-Marques; Ch. Finck et al. (HADES); Single-gap timing RPCs with bidimensional position-sensitive readout for very accurate TOF systems; Nuclear Instruments and Methods in Physics Research A (2003); 508:70-74

[Böh99] M. BöHMER (HADES); Das Auslesesystem für den Ringabbildenden Cherenkovdetektor im HADES Spektrometer (1999); Physik-Department der Technischen Universität München, Institut E12

[Böh01] M. Böhmer; T. Eberl; A. ElhardT et al. (HADES); Lepton identification with the CsI based RICH of HADES; Nuclear Instruments and Methods in Physics Research A (2001); 471:25-29

[Bre99] Thomas BRetz (HADES); Magnetfeldeigenschaften des Spektrometers HADES (1999); Diplomarbeit, Technische Universität München

[Fab03a] Laura FabBIETti (HADES); Study of the $e^{+} e^{-}$pair acceptance in the dilepton spectrometer HADES; Ph.D. thesis; Technische Universität München (2003)

[Fab03b] Laura Fabbietti; T. Eberl; J. Friese et al. (HADES); Photon detection efficiency in the CsI based HADES RICH; Nuclear Instruments and Methods in Physics Research A (2003); 502:256-260

[Fri03] J. Friese; R. Gernhäuser; P. Maier-Komor et al. (HADES); A new carbon based VUV mirror of righ radiation lenght for the HADES RICH; Nuclear Instruments and Methods in Physics Research A (2003); 502:241-245

[Frö02] InGo FröHLICH (HADES); Untersuchungen zur $\eta$-Produktion in pp-Reaktionen mit dem DISTO- und HADES-Spektrometer; Ph.D. thesis; II. Physikalisches Institut der Justus-Liebig-Universität Gießen (February 2002); URL http://pcweb.physik . uni-giessen.de/exp2

[Gar98] C. Garabatos et al. (HADES); Optimisation of low-mass drift chambers for HADES; Nuclear Instruments and Methods in Physics Research A (1998); 412:38-46

[GSI] GSI; GSI website; Gesellschaft für Schwerionenforschung; URL http://www.gsi.de

[HADa] HADES Simulation and Analysis Website; URL http://www-hades.gsi.de/ computing/

[HADb] HADES Collaboration; HADES website; http://www-hades.gsi.de

[HAD94] HADES Collaboration; A proposal for a High Acceptance Di-Electron Spectrometer (1994); GSI Darmstadt

[HAD06] HADES Collaboration; The HADES Wiki (2006); URL http://hades-wiki.gsi. de 
[IPN06] IPN website (November 2006); URL http://ipnweb.in2p3.fr/ rdd/HADES/index. html

[Kag00] M. Kagarlis; Pluto++: A Monte Carlo simulation tool for hadronic physics; GSI Report (2000); URL http://www-hades.gsi.de/computing/pluto/html/PlutoIndex. html

[Kas99] A. Kastenmüller; M. Böhmer; J. Friese et al. (HADES); Fast detector readout for the HADES-RICH; Nuclear Instruments and Methods in Physics Research A (1999); 433:438-443

[Kir07] Camilla KiRchHÜBEL; private communication (September 2007)

[Kol98] B. KolB et al.; HADES raw data event lengths (April 1998); see also: http://www-hades.gsi.de/docs/mdc/mdcana/mdc-essentials.htm; URL http:// www-hades.gsi.de/daq/event_lengths.html

[Leh99] Jörg Lehnert; Rene Becker; Ingo Fröhlich et al. (HADES); Ring recognition in the HADES second-level trigger; Nuclear Instruments and Methods in Physics Research A (1999); 433:268-273

[Leh00] JÖRG LEHNERT (HADES); Echtzeit-Mustererkennung zum Elektronennachweis mit einem RICH-Detektor in relativistischen Schwerionenkollisionen; Ph.D. thesis; II. Physikalisches Institut der Justus-Liebig-Universität Gießen (June 2000); URL http: //pcweb.physik.uni-giessen.de/exp2

[Leh03] Jörg Lehnert; Ingo Fröhlich; Wolfgang Kühn et al. (HADES); Performance of the HADES ring recognition hardware; Nuclear Instruments and Methods in Physics Research A (2003); 502:261-265

[Lin01] ERIK LiNS (HADES); Entwicklung eines Auslese- und Triggersystems zur Leptonenidentifizierung mit dem HADES-Flugzeitdetektor; Ph.D. thesis; II. Physikalisches Institut der Justus-Liebig-Universität Gießen (March 2001); URL http://pcweb. physik.uni-giessen.de/exp2

[Mar05] Jochen MARKERT (HADES); Untersuchung zum Ansprechverhalten der VieldrahtDriftkammern niedriger Massenbelegung des HADES Experimentes; Ph.D. thesis; Fachbereich Physik der Johann Wolfgang Goethe-Universität (2005)

[Mün04] C. Müntz; Jochen Markert; G. N. Agakichiev et al. (HADES); The HADES tracking system; Nuclear Instruments and Methods in Physics Research A (2004); $535: 242-246$

[Pec06] V. N. Pechenov (HADES); private communication (November 2006)

[Per07] Tiago Perez; Ming Liu et al.; private communication (September 2007)

[Pet00] Markus Petri (HADES); Entwicklung eines kombinierten Auslese- und EchtzeitTriggersystems zum Nachweis von Elektronen/Positronen-Signaturen in einem elektromagnetischen Schauerdetektor; Ph.D. thesis; II. Physikalisches Institut der JustusLiebig-Universität Gießen (August 2000); URL http://pcweb.physik. uni-giessen . de/exp2 
[Rel00] Relativistic Nuclear Physics: from Hundreds MeV to TeV: Proc.of Int. Workshop, Stara Lesna, Slovak Republic; THE SPECTROMETER HADES TRACKING SYSTEM

[Rus06] Anar Rustamov (HADES); Exclusive $\eta$ Meson Reconstruction in Proton-Proton Collisions at $2.2 \mathrm{GeV}$ with the HADES Spectrometer and High Resolution Tracking; Ph.D. thesis; Fachbereich Physik der Technischen Universität Darmstadt (July 2006); URL https://www-new.gsi.de/gsitools

[Sal02] Piotr Salabura (HADES); Vector Mesons in Stronly Interacting Systems (The Marian Smoluchowski Institute of Physics of the Jagellonian University, ul. Reymonta 4, 30-059 Kraków, Poland, 2002)

[Sch95] HEIKE ScHÖn (HADES); HADES Ein Dielektronenspektrometer mit hoher Akzeptanz für relativistische Schwerionenkollisionen; Ph.D. thesis; Johann-Wolfgang-Goethe Universität in Frankfurt am Main, Institut für Kernphysik (1995)

[Sch06] Alexander Schmah (HADES); The HADES Wiki - Pictures and Figures (nov 2006); URL http://hades-wiki.gsi.de

[SG03a] Manuel Sánches García; Momentum Reconstruction and Pion Production in the HADES Spectrometer at GSI; Ph.D. thesis; Universidade de Santiago de Compostela, Facultad de Física (April 2003)

[SG03b] Manuel SáncheZ-García (HADES); Momentum reconstruction and pion production analysis in the HADES spectrometer at GSI; Ph.D. thesis; Universidade de Santiago de Compostela (2003)

[Sko07] P. Skott et al.; A General Purpose Trigger and Readout Board for HADES and FAIR-Experiments (May 2007); Presented at 15th IEEE Real Time Conference 2007 (RT 07), Batavia, Illinois, 29 Apr - 4 May 2007.

[Str98] J. Stroth (HADES); A Low-mass Tracking System for HADES; in WCC 98 (1998) URL http://www-hades.gsi.de/mdc/talks/vienna98/vienna98.ppt

[Toi03] Alberica Toia; Ingo Fröhlich; Wolfgang Kühn et al. (HADES); A highly selective dilepton trigger based on ring recognition; Nuclear Instruments and Methods in Physics Research A (2003); 502:270-274

[Toi04] AlBerica ToIA (HADES); Performance of the HADES Spectrometer for Dilepton Identification in the Reaction $C+C$ at $1 \mathrm{AGeV}$; Ph.D. thesis; II. Physikalisches Institut der Justus-Liebig-Universität Gießen (March 2004); URL http://pcweb.physik. uni-giessen.de/exp2

[Tra01] Michael Traxler (HADES); Real-Time Dilepton Selection for the HADES Spectrometer; Ph.D. thesis; II. Physikalisches Institut der Justus-Liebig-Universität Gießen (2001); URL http://pcweb.physik.uni-giessen.de/exp2

[Tra06a] Michael TRAXLER; private communication (2006); HADES trigger expert 
[Tra06b] Michael Traxler; Ingo Fröhlich; M. Kajetanowicz et al.; A General Purpose Trigger and Readout Board (TRB) for HADES and FAIR-Experiments; Technical Report; GSI (2006); GSI Scientific Report; URL http://www.gsi.de/informationen/ wti/library/scientificreport2006

[Tra07] Michael Traxler; Ingo Fröhlich; Attilio Tarantola et al.; TRB Publication List in HADES Wiki; Technical Report; GSI (July 2007); URL http://hadeswiki.gsi.de/cgi-bin/view/DaqSlowControl/TRBPublicationList

[Zei99a] K. Zeitelhack; A. Elhardt; J. Friese et al. (HADES); The HADES RICH detector; Nuclear Instruments and Methods in Physics Research A (1999); 433:201-206

[Zei99b] K. Zeitelhack et al. (HADES); The HADES RICH detector; Nuclear Instruments and Methods in Physics Research A (1999); 433:201-206

[Zen97] Alfred ZENTEK (HADES); Konzeptionelle Untersuchungen für die HADESDriftkammern am Prototyp 0 (1997); Diplomarbeit, Fachbereich Physik der Johann Wolfgang Goethe-Universität

\section{Experimental Bibliography}

[Ach05] P. ACHARD et al.; Measurement of the running of the electromagnetic coupling at large momentum-transfer at LEP; Physics Letters B (2005); 623:26-36; doi:10.1016/j.physletb.2005.07.052

[Afa07] S. AfanAsiev et al. (PHENIX Collaboration); Enhancement of the dielectron continuum in $\sqrt{s_{N N}}=200 \mathrm{GeV} A u+A u$ collisions (2007); arXiv:0706.3034; URL http://arxiv.org/abs/0706.3034

[Aga05] G. AgAKICHIEv et al.; $e^{+} e^{-}$-pair production in Pb-Au collisions at $158 \mathrm{GeV}$ per nucleon; Eur. Phys. J. (2005); C 41:475-513

[Arn06] R. Arnaldi; R. Averbeck; K. Banicz et al.; First Measurement of the $\rho$ Spectral Function in High-Energy Nuclear Collisions; Physical Review Letters (Apr 2006); 96(162302); doi:10.1103/PhysRevLett.96.162302

[Bar85] L.M. BARKov et al.; Electromagnetic Pion Formfactor in the Timelike Region; Nuclear Physics B (1985); 256:365-384

[Bru87] R. BRun et al.; GEANT3 (1987); CERN-DD/EE/84-1

[Čer37a] Pavel Alekseyevich Čerenkov; The visible glow of pure liquids under the action of gamma-rays; Dokl. Akad. Nauk. SSSR [Sov. Phys. Dokl.] (1937); 2:37; translation of original paper

[Čer37b] Pavel Alekseyevich Čerenkov; Visible Radiation Produced by Electrons Moving in a Medium with Velocities Exceeding that of Light; Physical Review Letters (Aug 1937); 52(4):378-379; doi:10.1103/PhysRev.52.378

[Che06] S. CheKanov et al. (ZEUS); Jet-radius dependence of inclusive-jet cross sections in deep inelastic scattering at HERA (2006); arXiv:hep-ex/0701039 
[Eid04] S. Eidelman et al.; Review of Particle Physics; Physics Letters B (2004); 592; URL http://pdg.lbl.gov

[GEA] Detector Description and Simulation Tool; CERN Program Library Long Writeup W5013; http://wwwasdoc.web.cern.ch/wwwasdoc/geant_html3/geantall.html

[Gut06] H. H. GutBrod et al. (Editors); FAIR Baseline Technical Report (Gesellschaft für Schwerionenforschung, 2006); ISBN 3-9811298-0-6

[Har01] Olaf Hartmann et al.; Annual Report 2001 of GSI, GSI Future: A Detector for Antiproton Physiks (2001); URL http://www-linux.gsi.de/ charme/hesr

[Mas95] M. Masera et al. (HELIOS/3); Dimuon Production below Mass $3.1 \mathrm{GeV} / \mathrm{c} 2$ in $p$ - W and S-W Interactions at $200 \mathrm{GeV} / \mathrm{c} / \mathrm{A}$; Nuclear Physics A590 (1995); pages 93-102

[Maz94] M. A. Mazzoni et al.; Dimuon and vector-meson production in $p$ - $W$ and $S$ - $W$ interactions at $200 \mathrm{GeV} / \mathrm{c} /$ nucleon; Nuclear Physics (1994); A566:95c-102

[Nis00] RichaRD Nisius; The photon structure from deep inelastic electron photon scattering; Physics Reports (2000); 332:165-317

[Por97] R. J. Porter; S. Beedoe; R. Bossingham et al.; Dielectron Cross Section Measurements in Nucleus-Nucleus Reactions at 1.0A GeV; Phys. Rev. Lett. (Aug 1997); 79(7):1229-1232; doi:10.1103/PhysRevLett.79.1229

[Pov06] Bagdan Povh; Klaus Rith; Christipoh Scholz et al.; Particles and Nuclei An Introduction to the Physical Concepts (Springer-Verlag, 2006); 5th edition; ISBN 978-3-54036683-6

[Ros50] M. N. Rosenbluth; High Energy Elastic Scattering of Electrons on Protons; Physical Review (August 1950); 79(4):615

[Seg94] J. Seguinot and T. Ypsilantis; A historical survey of ring imaging Cherenkov counters; Nuclear Instruments and Methods in Physics Research A (1994); 343:1-29

[Seg99] J. Seguinot and T. Ypsilantis; Evolution of the RICH technique; Nuclear Instruments and Methods in Physics Research A (1999); 433:1-16

[Stö98] Horst Stöcker; Taschenbuch der Physik (Verlag Harri Deutsch, 1998); 3rd edition; ISBN 3-8171-1556-3

[TRI06] TRIGA; The TRIGA website (2006); http://triga.ga.com/

[Trn05] D. TRNKA et al. (CBELSA/TAPS Collaboration); Observation of In-Medium Modifications of the $\omega$ Meson; Physical Review Letters (2005); 94:192303; doi:10.1103/PhysRevLett.94.192303

[Trn06] DAVID TRNKA; Investigation of in-medium modifications of the $\omega$ meson in photonuclear reactions; Ph.D. thesis; II. Physikalisches Institut, Justus-LiebiegUniversität Gießen; Heinrich-Buff-Ring 16, D-35392 Gießen, Germany (May 2006); http://pcweb.physik.uni-giessen.de/taps/publications/theses/David_Trnka_Diss.pdf 
[Yao06a] W.-M. Yao et al.; Review of Particle Physics; Journal of Physics G (2006); 33:3194; Summary Tables of Particle Properties; doi:10.1088/0954-3899/33/1/001; URL http://pdg.lbl.gov

[Yao06b] W.-M. Yao et al.; Review of Particle Physics; Journal of Physics G (2006); 33:110118; Quantum Chromodynamics and its Coupling; doi:10.1088/0954-3899/33/1/001; URL http://pdg.lbl.gov

[Yao06c] W.-M. YaO et al.; Review of Particle Physics; Journal of Physics G (2006); 33:97; Physical Constants; doi:10.1088/0954-3899/33/1/001; URL http://pdg.lbl.gov

[Yao06d] W.-M. Yao et al.; Review of Particle Physics; Journal of Physics G (2006); 33:1-1232; doi:10.1088/0954-3899/33/1/001; URL http://pdg.1bl.gov

[Yao06e] W.-M. Yao et al.; Review of Particle Physics; Journal of Physics G (2006); 33:181-194; Structure Functions; doi:10.1088/0954-3899/33/1/001; URL http://pdg.lbl.gov

[Yao06f] W.-M. YaO et al.; Review of Particle Physics; Journal of Physics G (2006); 33:271292; Particle Detectors; doi:10.1088/0954-3899/33/1/001; URL http://pdg.1bl.gov

[Yao06g] W.-M. Yao et al.; Review of Particle Physics; Journal of Physics G (2006); 33:258-270; Passage of Particles through Matter; doi:10.1088/0954-3899/33/1/001; URL http: $/ /$ pdg.lbl.gov

[Yao06h] W.-M. YaO et al.; Review of Particle Physics; Journal of Physics G (2006); 33:165172; Quark Model; doi:10.1088/0954-3899/33/1/001; URL http://pdg.1bl.gov

[Yps94] T. Ypsilantis and J. Seguinot; Theory of ring imaging Cherenkov counters; Nuclear Instruments and Methods in Physics Research A (1994); 343:30-51

\section{RFC Bibliography}

[RFC87] RFC 1011 - Official Internet Protocols (May 1987); URL http://rfc.net/rfc1011. $\mathrm{html}$

[RFC92] RFC 1311 - Introduction to the STD Notes (March 1992); URL http://rfc.net/ rfc1311.html

\section{STD Bibliography}

[STD80] STD 6 - User Datagram Protocol (August 1980); URL http://rfc.net/std6.htm]

[STD81a] STD 5 - Internet Protocol (September 1981); URL http://rfc.net/std5.html

[STD81b] STD 7 - Transmission Control Protocol (September 1981); URL http://rfc.net/ std7.html

[STD82] STD 37 - An Ethernet Address Resolution Protocol - or - Converting Network Protocol Addresses to 48.bit Ethernet Address for Transmission on Ethhernet Hardware (November 1982); URL http://rfc.net/std37.html 
[STD84] STD 41 - A Standard for the Transmission of IP Datagrams over Ethernet Networks (April 1984); URL http://rfc.net/std41.html

[STD88a] STD 42 - A Standard for the Transmission of IP Datagrams over Experimental Ethernet Networks (February 1988); URL http://rfc.net/std42.html

[STD88b] STD 43 - A Standard for Transmission of IP Datagrams over IEEE 802 Networks (February 1988); URL http://rfc.net/std43.html

[STD89] STD 3 - Requirements for Internet Hosts - Communication Layers (October 1989); URL http://rfc.net/std3.html

[STD04] STD 1 - Internet Official Protocol Standards (July 2004); URL http://rfc.net/ std1.html

[STD06] STD Index (April 2006); URL http://rfc.net/std-index.html

\section{Technical Bibliography}

[Ana02] Analog Devices, Inc; One technology Way, Norwood, Mass. 02062-9106; TigerSHARC DSP Microcomputer ADSP-TS101S Preliminary Datasheet; Rev.PrE edition (February 2002); URL http://www.analog.com

[Ana04] Analog Devices, Inc; One technology Way, Norwood, Mass. 02062-9106; ADSP-TS101 TigerSHARC Processor Hardware Reference; revision 1.1 edition (May 2004); Part Number 82-001996-01; URL http: //www.analog.com

[Ash01] Peter J. Ashenden; The Designers Guide to VHDL (Morgan Kaufmann Publishers, 2001); 2nd edition; ISBN 1558606742

[ATM00] ATM; The ATM Forum, Worldwide Headquarters 2570 West El Camino Real, Suite 304, Mountain View, CA 94040-1313; The ATM Forum Technical Committee Framebased ATM Interface (Level 3) (March 2000); URL http://www.atm.org

[Big91] Ezio Biglieri; Pariush Divsalar; Peret J. McLane et al.; Introduction to TrellisCoded Modulation with Applications (Prentice-Hall, Inc., Upper Saddle River, NJ, USA, 1991); ISBN 0023099658

[Bru97] Rene Brun and Fons Rademakers; ROOT - An object oriented data analysis framework (1997); (389):81-86; doi:10.1016/S0168-9002(97)00048-X

[Bru07] Rene Brun and Fons Rademakers; The ROOT Website (2007); URL http://root. cern.ch

[Cad06] Cadence Design Systems; NC-Sim (2006); URL http://www.cadence.com

[Ce107] Celoxica; The Celoxica Website (September 2007); URL http://www.celoxica.com

[Com07] Gerald Combs et al.; Wireshark Website (February 2007); URL http://www . wireshark.org 
[Coo01] JR. Coombs and F. Clyde; Printed Circuits Handbook; McGraw-Hill; 5th edition (2001)

[Cyp01] Cypress Semiconductor Corporation; 3901 North First Street, San Jose, CA 95134; Delta39K ISR CPLD Family; rev. c edition (December 2001); URL http://www. cypress.com

[Cyp02] Cypress Semiconductor; 9901 North First Street, San jose, CA 95134, (408) 943-2600; Warp HDL Development System Users Guide (May 2002); URL http://www. cypress . $\mathrm{com}$

[For73] G. D. JR ForneY; The Viterbi Algotithm; Proceeding IEEE (March 1973); 61:268-278

[Gra93] Martin Graham; High-Speed Digital Design, A Handbook of Black Magic (Prentice Hall, 1993)

[H50] H500B (2/00), 1000Base-T Magnetics Module; URL http://www.pulseeng.com

[Her02] Richard Herveille; Wade D. Peterson et al.; Specification for the: WISHBONE System-on-Chip (SoC) Interconnection Architecture for Portable IP Cores (OpenCores Organization, 2002); revision: b.3 edition; URL http://www.opencores.org/ projects.cgi/web/wishbone/wishbone

[Hor89] Paul Horowitz and Winfield Hill; The Art of Electronics (Cambridge University Press, 1989); 2nd edition

[Hun95] Craig Hunt; TCP/IP Netzwerk Administration (O'Reilly/International Thomson Verlag GmbH und Co KG, 1995); 1st edition

[IEE00] IEEE Computer Society; 802.3; IEEE Standards (The Institue of Electrical and Electronics Engineers, Inc, 3 Park Avenue, New York, NY 10016-5997, USA, 2000); revision 2000 edition; URL http://www. ieee802.org

[IEE02] 1076 IEEE Standard VHDL Language Reference Manual; ieee std 1076-2002 edition (May 2002); URL http://www.ieee.com

[Ins02] The Institute of Electrical and Electronics Engineers, Inc; 3 Park Avenue, New York, NY 10016-5997, USA; IEEE Std 802.3; 2002 edition (August 2002); URL http://www . ieee.org

[LSN] Single Output LSN D5 Models; URL http://www.datel.com

[Nat01] National Semiconductor Corporation; DP83861 VQM-3 EN Gig PHYTER 10/100/1000 Ethernet Physikal Layer; 1st edition (April 2001); URL http://www. national.com

[Ora06] Oracle Corporation; The Oracle Website (2006); URL http://www.oracle.com

[Pet93] Wade D. Peterson; The VMEBus Handbook (Scottsdale - Zaltbommel, 1993); 3rd edition 
[PMC00] PMC-Sierra, Inc; 105-8555 Baxter Place Burnaby, BC, Canada V5A 4V7; POSPHY-Level 3 Saturn Compatible Packet over SONET Interface Specification for Physical und Link Layer Devices; pmc-1980495, issue 4 edition (June 2000); URL http: //www.pmc-sierra.com

[PMC01] PMC-Sierra, Inc.; 105-8555 Baxter Place Burnaby, BC, Canada V5A 4V7; PM3386 S/UNI-2xGE Dual Gigabit Ethernet Controller Datasheet; issue 7 edition (July 2001); URL http://www.pmc-sierra.com

[Rec00] JÖRG RECH; Jetzt klapts auch mit Kupfer; Heise Verlag; c't ausgabe 14 edition (July 2000)

[Sch97] Christian Schlegel and Lance PereZ; Trellis Coding (Wiley-IEEE Press, 97); 1st edition; ISBN 0780310527

[Syn06] Synplicity, Inc.; Synplify Pro (2006); URL http://www.synplicity.com/

[Tex00a] Texas Instruments; Post Office Box 655303 Dallas, Texas 75265; SN74CBT32245 32-Bit Fet Bus Switch; SCDS104B edition (April 2000); URL http://www.ti.com

[Tex00b] Texas Instruments; Post Office Box 655303 Dallas, Texas 75265; SN74LVTH32245 3.3$V$ ABT 32-Bit Bus Transceiver with 3-State Outputs; sbs750 edition (October 2000); URL http://www.ti.com

[Vis04] Compiler and Library Manual for TigerSHARC Processors; One technology Way, Norwood, Mass. 02062-9106; revision 1.1 edition (March 2004); Part Number: 82-000336-03; URL http://www.analog.com

[Vit79] A. J. Viterbi and K. Omuta; Principles of Digital Communication and Coding (MCGraw-Hill Book Company, New York, NY, 1979)

[VIT06] VITA; VMEbus International Trade Association (2006); URL http://www.vita.org

[Wie99] A. Wiemers; Multilayersysteme; Ilfa GmbH; 3.3 edition (August 1999); URL http: //www.ilfa.de

[Wis06] Wishbone PeOPle; Wishbone Website (2006); URL http://www.opencores.org/ projects.cgi/web/wishbone/wishbone

\section{Theoretical Bibliography}

[Bal00] Gunnar S. BALI; QCD forces and heavy quark bound states (May 2000); HUB-EP99/67, arXiv:hep-ph/0001312v2

[Bra98] E. L. Bratkovskaya; W. Cassing; R. Rapp et al.; Dilepton production and $m_{T}$-scaling at BEVALAC/SIS energies; Nuclear Physics A (1998); 634:168; doi:10.1016/S0375-9474(98)00135-3

[Bro91] G. E. Brown and Mannque Rho; Scaling effective Lagrangians in a dense medium; Phys. Rev. Lett. (May 1991); 66(21):2720-2723; doi:10.1103/PhysRevLett.66.2720 
[Bro02] G.E. Brown and Mannque Rho; On the manifestation of chiral symmetry in nuclei and dense nuclear matter; Physics Reports (2002); 363:85 - 171; doi:10.1016/S03701573(01)00084-9

[Cas95] W. Cassing; W. Ehehalt and C. M. Ko; Dilepton Production at SPS Energies; Physics Letters B (1995); 363:35; arXiv:hep-ph/9508233

[Cha96] G. Chanfray; R. Rapp and J. Wambach; Medium Modifications of the Rho Meson at CERN Super Proton Synchrotron Energies (200 GeV/nucleon); Phys. Rev. Lett. (Jan 1996); 76(3):368-371; doi:10.1103/PhysRevLett.76.368

[Cle98] J. Cleymans and K. Redlich; Unified Description of Freeze-Out Parameters in Relativistic Heavy Ion Collisions; Phys. Rev. Lett. (Dec 1998); 81(24):5284-5286; doi:10.1103/PhysRevLett.81.5284

[Ger89] P. Gerber and H. Leutwyler; Hadrons below the chiral phase transition; Nuclear Physics B (July 1989); 321(2):387-429; doi:10.1016/0550-3213(89)90349-0

[GM68] Murray Gell-Mann; R. J. Oakes and B. Renner; Behavior of Current Divergences under $S U_{3} \times S U_{3} ;$ Phys. Rev. (Nov 1968); 175(5):2195-2199; doi:10.1103/PhysRev.175.2195

[Hal84] Francis Halzen and Alan Douglas Martin; QUARKS AND LEPTONS: An Introductory Course in Modern Particle Physics (Wiley \& Sons, Inc., 1984); ISBN 0-47188741-2

[Hun97] C. M. Hung and E. V. Shuryak; Dilepton and/or photon production in heavy ion collisions and the QCD phase transition; Phys. Rev. C (Jul 1997); 56(1):453-467; doi:10.1103/PhysRevC.56.453

[Ich03] H. Ichie; V. Bornyakov; T. Streuer et al.; Flux Tubes of Two- and ThreeQuark System in Full QCD*; Nuclear Physics A721 (2003); pages 899c-902; doi:10.1016/S0375-9474(03)01238-7

[Jac99] John David JaCKson; Classical Electrodynamics (John Wiley \& Sons, Inc., 1999); 3rd edition; ISBN: 0-471-30932-X

[Kli90] S. Klimt; M. Lutz and W. Weise; Chiral phase transition in the SU(3) Nambu and Jona-Lasinio model; Physics Letters B (October 1990); 249(3,4):386; doi:10.1016/03702693(90)91003-T

[Kli96] F. KLingL; N. KAISER and W. Weise; Effective Lagrangian approach to vector mesons, their structure and decays; Z. Phys. A (1996); 356:193

[Koc95] Volker Koch; Introduction to Chiral Symmetry; LBNL-Report 38000 (1995); arXiv: nucl-th/9512029

[Koc02] Volker KocH; Aspects of Chiral Symmetry; LBNL-39463 UC-413 (March 2002); URL http://theory.gsi.de/ knoll/Lecture-notes/koch_notes.pdf 
[Li95] G. Q. Li; C. M. Ko and G. E. Brown; Enhancement of Low-Mass Dileptons in Heavy Ion Collisions; Phys. Rev. Lett. (Nov 1995); 75(22):4007-4010; doi:10.1103/PhysRevLett.75.4007

[Li96] G. Q. LI; C. M. Ko and G. E. Brown; Effects of in-medium vector meson masses on low-mass dileptons from SPS heavy-ion collisions; Nuclear Physics A (1996); 606:568; arXiv:nucl-th/9608040

[Noe18] Emmy Noether; Invariante Variationsprobleme; Nachr. d. König. Gesellsch. d. Wiss. zu Göttingen, Math-phys. Klasse (1918); pages 235-257; arXiv:physics/0503066v1

[Rap97] R. RAPP; G. Chanfray and J. WAMBACH; Rho meson propagation and dilepton enhancement in hot hadronic matter; Nuclear Physics A (1997); 617:472-495; doi:10.1016/S0375-9474(97)00137-1

[Rap00] R. RAPP and J. Wambach; Chiral Symmetry Restoration and Dileptons in Relativistic Heavy-Ion Collisions; ADV.NUCL.PHYS. (2000); 25:1; arXiv:hep-ph/9909229

[Sak69] J. J. SAkURAi; Currents and Mesons; The University of Chicago Press (1969)

[Tak03] T.T. TAKahashi et al.; Behind the Success of the Quark Model; Nuclear Physics A (2003); 721:926c-929; doi:10.1016/S0375-9474(03)01244-2

[Tas01] EnRICO TASsi; Measurement of dijet production in neutral current deep inelastic scattering at high $Q^{2}$ and determination of $\alpha_{S}$ at HERA; Ph.D. thesis; Universität Hamburg (2001); URL http://www-library.desy.de/cgi-bin/showprep.pl? desy-thesis-01-059

[Urb98] M. Urban; M. Buballa; R. Rapp et al.; Momentum Dependence of the Pion Cloud for Rho Mesons in Nuclear Matter; Nuclear Physics A (1998); 641:433; arXiv:nucl-th/ 9806030

[Urb00] M. Urban; M. Buballa; R. Rapp et al.; Modifications of the Rho Meson from the Virtual Pion Cloud in Hot and Dense Matter; Nuclear Physics A (2000); 673:357; arXiv:nucl-th/9910004 